\title{
Electrocortical maturation of healthy preterm infants: the feasibility of automated analysis
}

Citation for published version (APA):

Niemarkt, H. J. (2012). Electrocortical maturation of healthy preterm infants: the feasibility of automated analysis. [Doctoral Thesis, Maastricht University]. Datawyse / Universitaire Pers Maastricht. https://doi.org/10.26481/dis.20121026hn

Document status and date:

Published: 01/01/2012

DOI:

10.26481/dis.20121026hn

Document Version:

Publisher's PDF, also known as Version of record

\section{Please check the document version of this publication:}

- A submitted manuscript is the version of the article upon submission and before peer-review. There can be important differences between the submitted version and the official published version of record.

People interested in the research are advised to contact the author for the final version of the publication, or visit the DOI to the publisher's website.

- The final author version and the galley proof are versions of the publication after peer review.

- The final published version features the final layout of the paper including the volume, issue and page numbers.

Link to publication

\footnotetext{
General rights rights.

- You may freely distribute the URL identifying the publication in the public portal. please follow below link for the End User Agreement:

www.umlib.nl/taverne-license

Take down policy

If you believe that this document breaches copyright please contact us at:

repository@maastrichtuniversity.nl

providing details and we will investigate your claim.
}

Copyright and moral rights for the publications made accessible in the public portal are retained by the authors and/or other copyright owners and it is a condition of accessing publications that users recognise and abide by the legal requirements associated with these

- Users may download and print one copy of any publication from the public portal for the purpose of private study or research.

- You may not further distribute the material or use it for any profit-making activity or commercial gain

If the publication is distributed under the terms of Article $25 \mathrm{fa}$ of the Dutch Copyright Act, indicated by the "Taverne" license above, 


\section{Electrocortical maturation of healthy preterm infants:}

the feasibility of automated analysis 
Printing of this thesis was financially supported by:

Chiesi Pharmaceuticals Nederland BV

Nemo Healthcare

Stichting ter Bevordering van de Kindergeneeskunde Maastricht

Stichting Research Neonatologie Veldhoven

Vygon Nederland BV

Wim van der Leeden

(c) Copyright H.J. Niemarkt, Maastricht 2012

No parts of this thesis may be reproduced or transmitted in any form or by any means, without permission of the author.

ISBN:

9789461591685

Layout/Cover by: Lina Meisen | www.neondot.com

Printed by: Datawyse Maastricht | Universitaire Pers Maastricht 


\section{Electrocortical maturation of healthy preterm infants: the feasibility of automated analysis}

\section{PROEFSCHRIFT}

ter verkrijging van de graad van doctor aan de Universiteit Maastricht

op gezag van Rector Magnificus,

Prof. dr. L.L.G. Soete, volgens het besluit van het

College van Decanen,

in het openbaar te verdedigen

op vrijdag 26 oktober 2012 om 14:00 uur

door

Hendrik Johannes Niemarkt

geboren op 16 februari 1978

te Heerlen

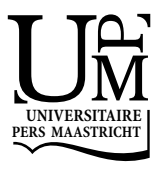




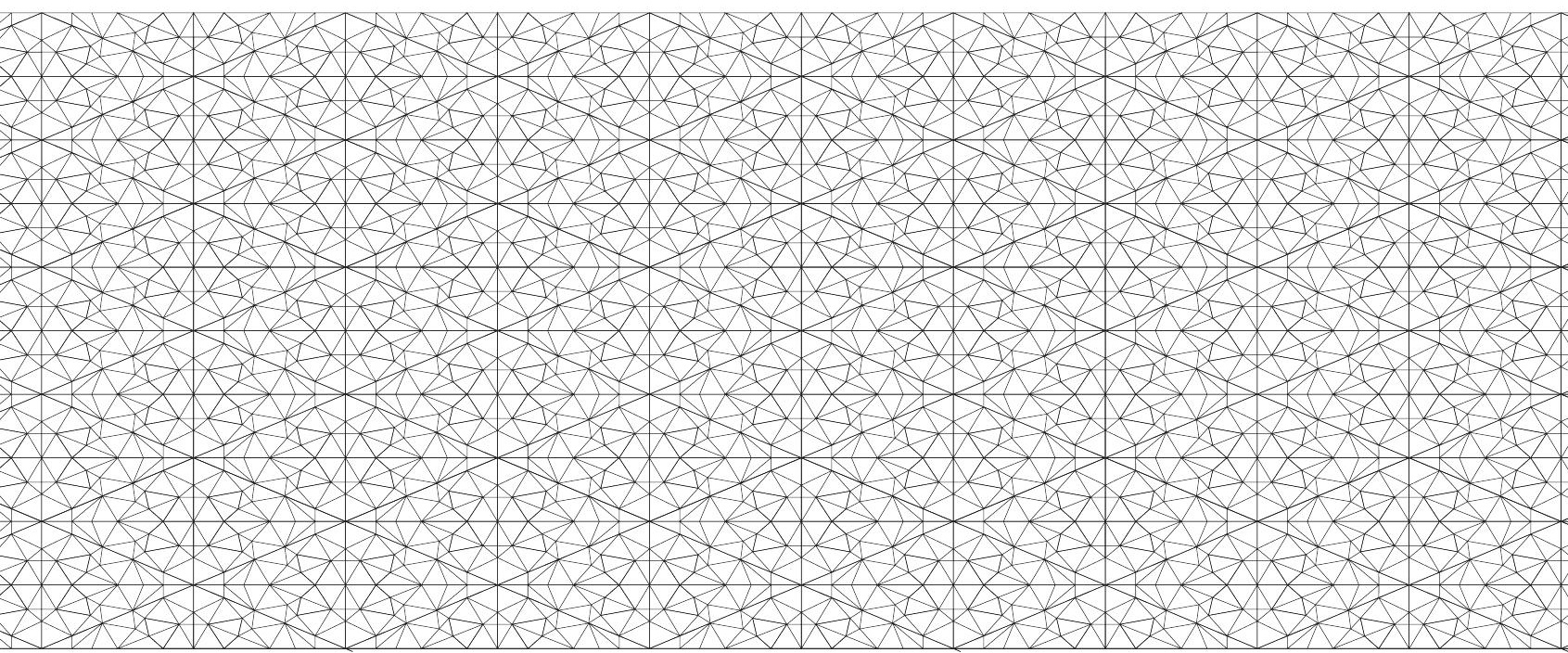

\section{Promotores}

Prof. dr. L.J.I. Zimmermann

Prof. dr. S. Bambang Oetomo (Technische Universiteit Eindhoven, Máxima Medisch (entrum Veldhoven)

Prof. dr. B.W. Kramer

\section{Copromotor}

Dr. P. Andriessen (Máxima Medisch Centrum Veldhoven)

\section{Beoordelingscommissie}

Prof. dr. W.H. Mess (voorzitter)

Prof. dr. A.F. Bos (Beatrix Kinderziekenhuis, Universitair Medisch Centrum Groningen)

Prof. dr. T. Delhaas

Dr. J. Nicolai

Prof. dr. L.S. de Vries (Wilhelmina Kinderziekenhuis, Universitair Medisch Centrum Utrecht) 


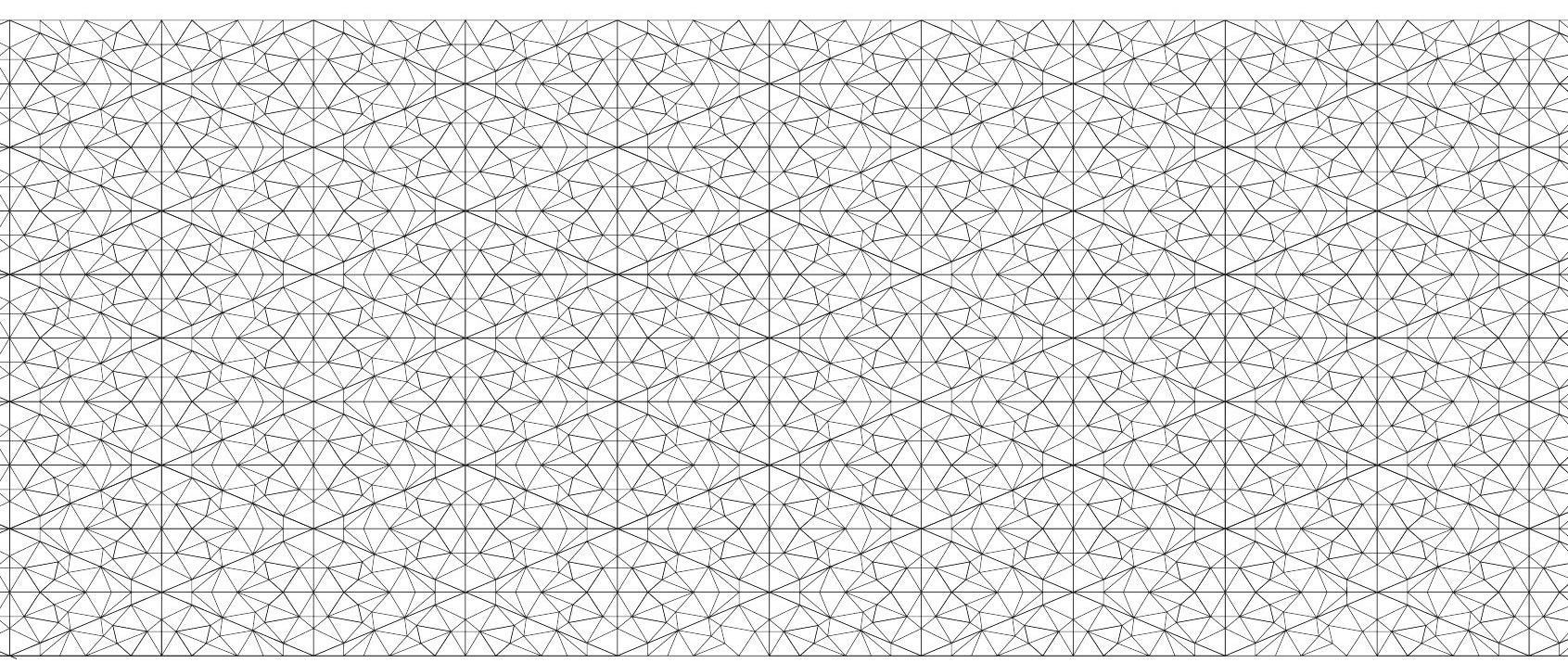




\section{Contents}

\section{List of abbreviations}

\section{Introduction and review}

\section{Chapter 1}

Introduction

\section{$31 \quad$ Chapter 2}

Analyzing EEG maturation in preterm infants: The value of a quantitative approach Journal of neonatal-perinatal medicine 2008 Jan; 1(3): 131-44

\section{EEG section}

\section{Chapter 3}

Quantitative analysis of maturational changes in EEG background activity in very preterm infants with a normal neurodevelopment at 1 year of age

Early Hum Dev. 2010 Apr;86(4):219-24

\section{$71 \quad$ Chapter 4}

Automatic burst detection for the EEG of the preterm infant

Physiol Meas. 2011 0ct;32(10):1623-37

\section{$91 \quad$ Chapter 5}

Maturational Changes in Automated EEG Spectral Power Analysis in Preterm Infants Pediatr Res. 2011 Nov;70(5):529-534

\section{Chapter 6}

Topography of maturational changes in EEG burst spectral power of the preterm infant with a normal follow-up at 2 years of age

Clin Neurophysiol. 2012 May 26. [Epub ahead of print] 


\section{$\underline{\text { Amplitude-integrated EEG (aEEG) section }}$}

\section{Chapter 7}

Quantitative analysis of amplitude-integrated electroencephalogram patterns in stable preterm infants, with normal neurological development at one year

Neonatology. 2010;97(2):175-82

\section{Chapter 8}

Multi-channel amplitude-integrated EEG characteristics in preterm infants with a normal neurodevelopment at two years of corrected age

Early Hum Dev. 2012;88(4):209-16

\section{$\underline{\text { Discussion and Dutch summary }}$}

\section{Chapter 9}

Discussion and future perspectives

\section{Nederlandse samenvatting}

\section{Appendices}

\section{Dankwoord}

\section{Curriculum Vitae}

\section{List of publications}

\section{List of co-authors}




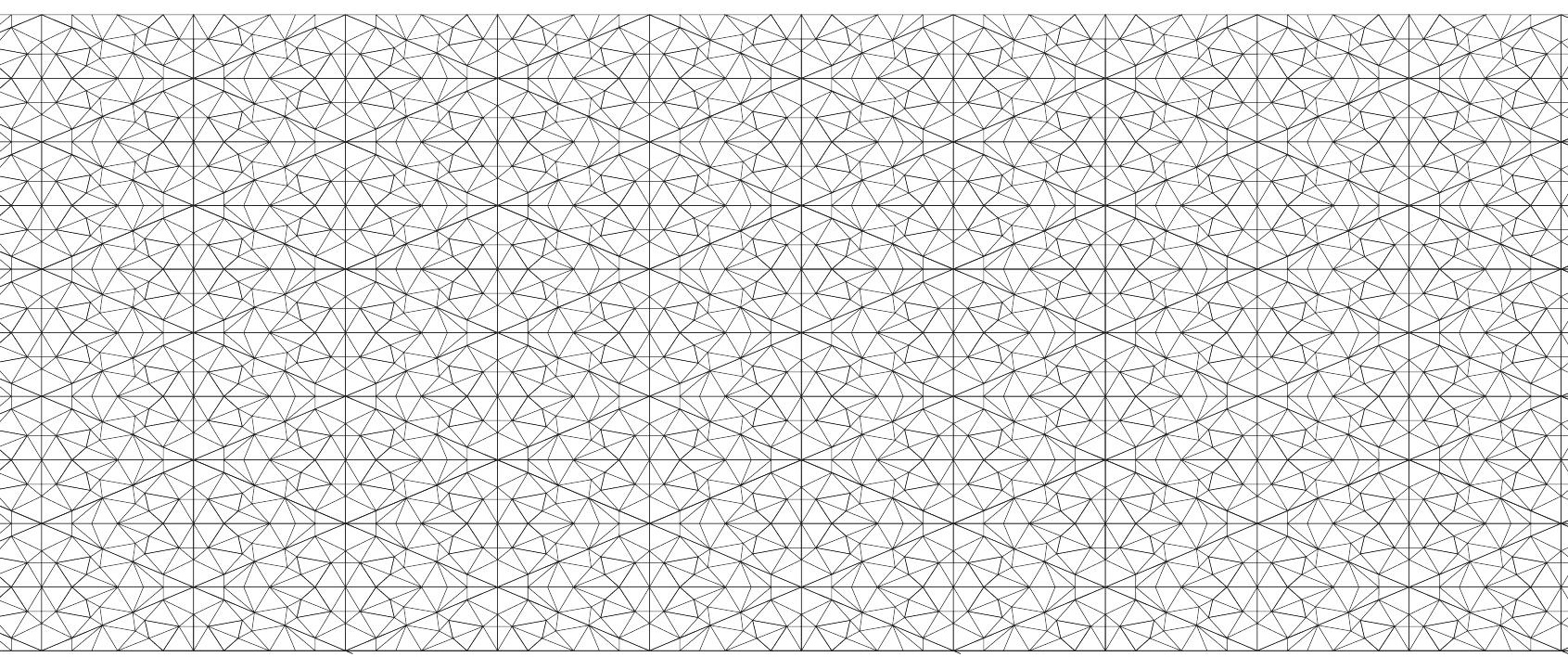




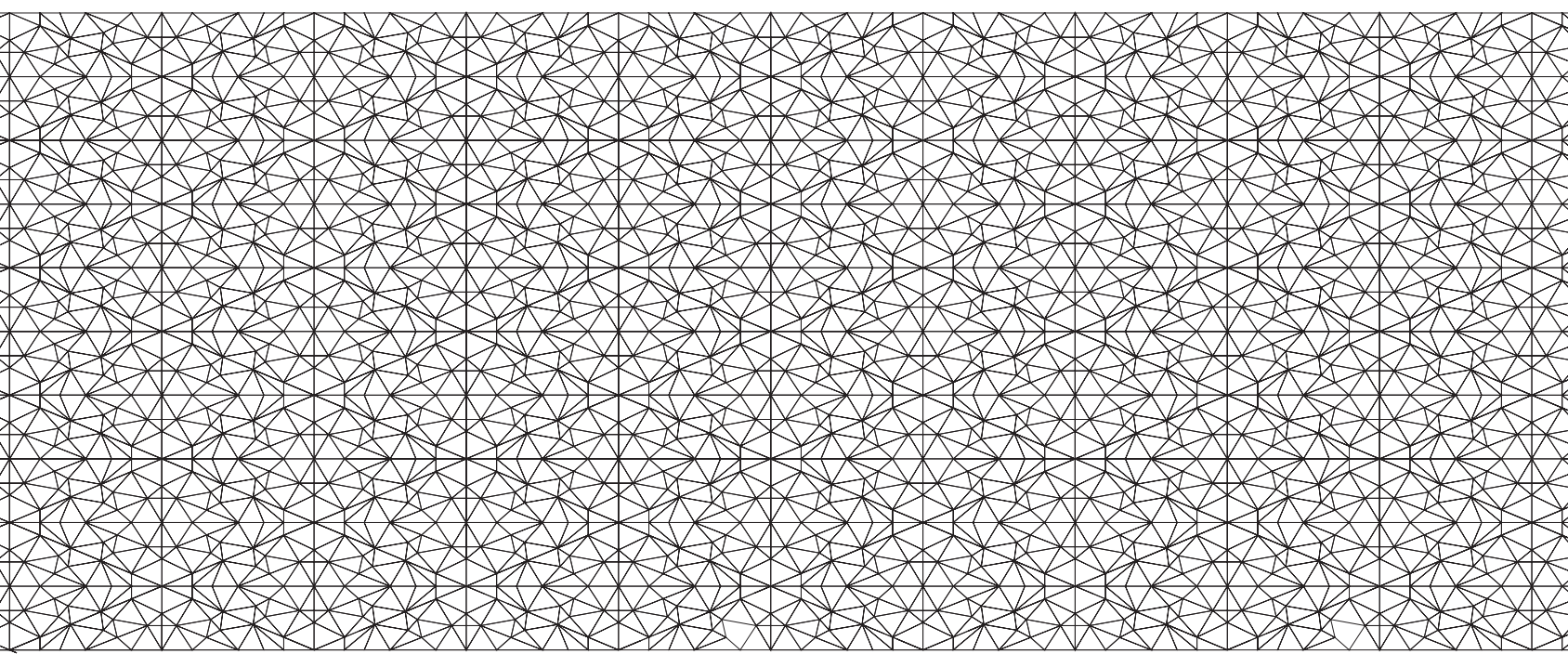

\section{List of abbreviations}

$\begin{array}{llll}\text { PMA } & \text { Postmenstrual Age } & \text { NREM } & \text { Non Rapid Eye Movement } \\ \text { GABA } & \gamma \text {-aminobutyric acid } & \text { IBR } & \text { Interburst Burst Ratio } \\ \text { MRI } & \text { Magnetic Resonance Imaging } & \text { PA } & \text { Postnatal Age } \\ \text { DTI } & \text { Diffusion Tensor Imaging } & \text { BSID-II-NL } & \text { Bayley Scales of Infant Development } \\ \text { ADC } & \text { Apparent Diffusion Coefficient } & & \text { II Dutch Version } \\ \text { EEG } & \text { Electroencephalogram } & \text { IVH } & \text { Intraventricular Hemorrhage } \\ \text { IBI } & \text { Interburst Interval } & \text { PVL } & \text { Periventricular Leucomalacia } \\ \text { REM } & \text { Rapid Eye Movements } & \text { CTH } & \text { Channel Threshold High } \\ \text { SAT } & \text { Spontaneous Activity Transient } & \text { CTL } & \text { Channel Threshold Low } \\ \text { aEEG } & \text { Amplitude-integrated } & \text { ATL } & \text { Amplitude Threshold Low } \\ & \text { Electroencephalogram } & \text { ATH } & \text { Amplitude Threshold High } \\ \text { NICU } & \text { Neonatal Intensive Care Unit } & \text { PPV } & \text { Positive Predictive Value } \\ \text { LMA } & \text { LowerMargin Amplitude } & \text { MEG } & \text { Magneto Encephalogram } \\ \text { UMA } & \text { UpperMargin Amplitude } & \text { SEF } & \text { Spectral Edge Frequency } \\ \text { BW } & \text { Bandwidth } & \text { IQR } & \text { Interquartile Range } \\ \text { GA } & \text { Gestational Age } & \text { MDI } & \text { Mental Developmental Index } \\ \text { BS } & \text { Burst Suppression } & \text { PDI } & \text { Psychomotor Developmental Index } \\ \text { FFT } & \text { Fast Fourier Transform } & \text { DC-\% } & \text { Percentage Discontinuous Activity }\end{array}$





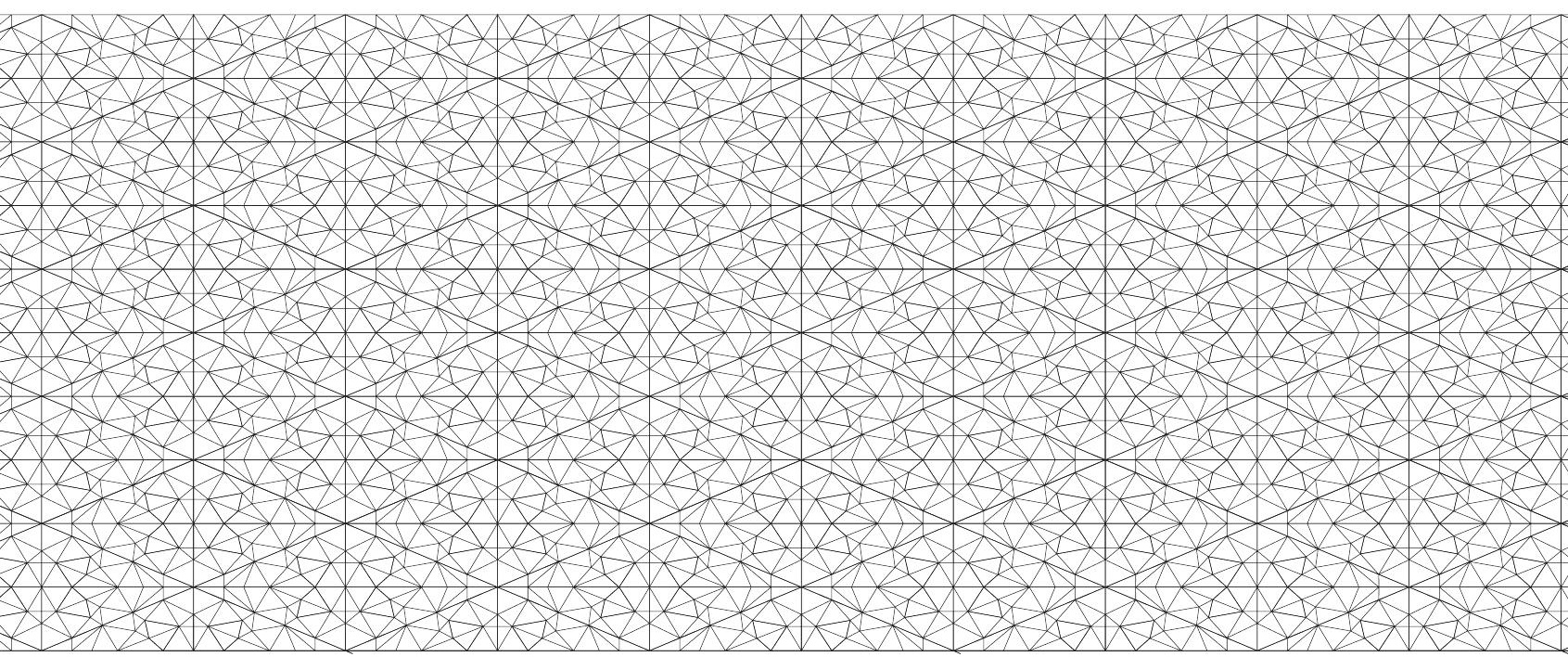




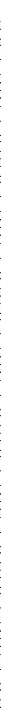

Introduction 


\section{Neurodevelopment}

Advances in the care for preterm infants have led to an increased survival. However, a large number of these infants still experience neurological deficits later in life, even in the absence of neurosonographic abnormalities [1-3]. The exact etiology of these neurodevelopmental deficits remains to be clarified, but it is suggested that maternal and several medical, environmental and iatrogenic conditions may interfere with the development of the vulnerable preterm brain [4-6]. Therefore, a better understanding of neurodevelopment in preterm infants and knowledge of the factors interfering with brain development are of great importance.

Brain development has been studied extensively in animals and humans [7-10]. The first steps in brain development include proliferation of neurons, oligodendrocytes (myelination) and astrocytes (regulatory cells and modulators of synapse formation) (5-40 weeks postmenstrual age = PMA), migration (8-28 weeks PMA) and cortical folding (12 weeks-3 years) [11, 12].

Figure 1:

Neural proliferation

Neuronal migration

Presence Subplate

Axon \& dendrite sprouting

Synapse formation

Glial Cell proliferation

Myelination

Programmed cell death

Axon retraction

Synapse elimination

Acetylcholaminergic systems

Catecholaminergic systems

GABA

Glutamate/Aspartate

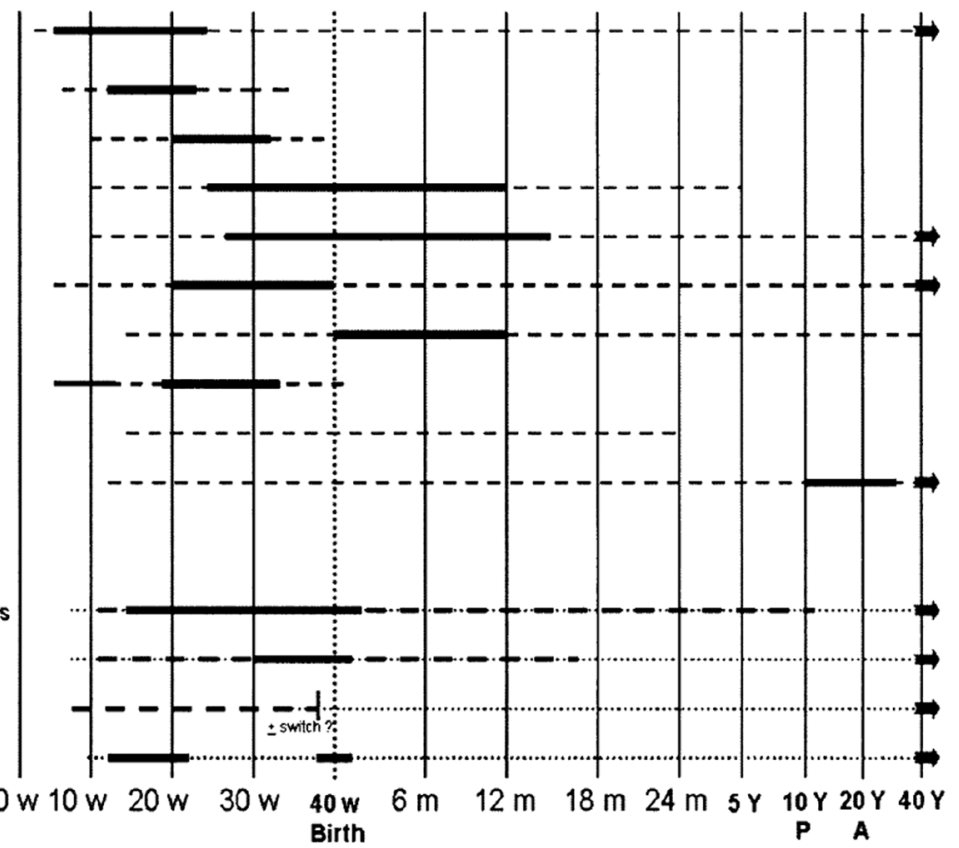

Figure 1 modified from [11] Summary of timing of neurobiological processes in the telencephalon during human ontogeny. Note that the time axis at the bottom of the figure is an arbitrary one. $\mathrm{W}=$ weeks $\mathrm{PMA}, \mathrm{M}=$ postnatal months, $\mathrm{Y}=\mathrm{years}, \mathrm{P}=$ onset of puberty, $A=$ onset of adulthood.

In the upper part of the figure, a broken line means that the process is active, a bold line indicates that the process is very active. In the lower part of the figure, the development of various neurotransmitter systems is represented. A thin broken line means that the transmitter is present; a continuous bold line represents a period of overexpression of the transmitter. The increasing dot density at the catecholaminergic systems denotes the gradual increase in dopaminergic activity. The bold broken line at the GABAergic systems reflects that GABA in early life exerts an excitatory function and later on switches to its adult inhibitory function. 
The subcortical plate serves as a waiting room for the projection (originating from the thalamus), commissural (from corpus callosum) and association fibers (other cortical sites). These fibers connect first with the subplate neurons before entering the cortex. Therefore, the subplate plays a major role in the connectivity between the cortical neurons and subcortical areas $[13,14]$. The subplate reaches maximum thickness at 28 weeks of gestation. At this timepoint, the process of synaptogenesis and myelination starts (figure 1) [11]. Besides, a late 'wave' of GABAergic interneurons is produced and migrates towards the outer layers of the cortex. These neurons form the hallmark of the human cortex. Beyond 32 weeks of gestation, the subplate gradually decreases by programmed cell death. Apoptosis of exuberant neurons also takes place in other parts of the brain and is a critical process in brain organization $[6,11]$.

Hence, an important part of neurodevelopment takes place in the third trimester. Very preterm infants pass through this important phase in an extra-uterine environment.

\section{Magnetic Resonance Imaging}

In the past two decades, magnetic resonance imaging (MRI) has proved to be a very useful tool in the investigation of structural brain development of preterm infants. With sequential MRI imaging of preterm infants, the development of sub-cortical and cortical gray matter, white matter and cortical folding has been demonstrated $[15,16]$. Furthermore, it has been shown that preterm infants have prominent reductions in cortical and sub-cortical gray matter, even in the absence of white matter abnormalities [17]. Abnormalities in gray and white matter of preterm infants persist during childhood [18-20] and are related to neurocognitive deficits [21-23]. Moreover, preterm birth may even influence brain development later in life [24]. On the other hand, neuro-protective strategies were found to protect brain development $[25,26]$.

With a relatively new MRI modality, diffusion tensor imaging (DTI), the diffusion of water particles is assessed [27]. The physical constant characterizing this water motion is called the apparent diffusion coefficient (ADC) [28]. The ADC is influenced by a wide range of complex factors, like overall tissue water content, relative volume fractions of the intra- and extra-cellular spaces, the energy status of cells and tissue microstructure (like myelination). Diffusion based MRI provides one of the earliest indicators of brain injury, long before this can be detected by other imaging techniques.

However, by assessing anisotropy, diffusion based MRI also has proved to be a useful technique to evaluate the microstructural state of white matter and the developing cortex. Anisotropy refers to the condition in which ADC values differ depending on the direction along which they are measured. In myelinated white matter the movement of water particles is higher parallel to the fibers than perpendicular to the fibers. This leads to high degrees of anisotropy. During preterm development anisotropy increases in the white matter, which is not only influenced by myelination, but also by changes in the structure of the axons, the increase in oligodendrocytes and changes in $\mathrm{Na}+/ \mathrm{K}+-$ ATPase activity. Cortical gray matter anisotropy increases in early development, reflecting the radial organization of the developing cortex. Beyond 32 weeks, the gray matter anisotropy decreases. This is due to the appearance of interneurons and elaboration of pyramidal cells, forming neuronal networks $[27,28]$. 
Not only the degree of anisotropy can be characterized (by relative or fractional anisotropy) but also the direction in which the water diffusion (ADC) is greatest (major eigenvector). The direction along which water ADC values are greatest (major eigenvector) represents the primary orientation of the myelinated fibers. This can be used to detect the developing cerebral white matter tracts (fiber tracking) [28]. DTI imaging has proven to be a good tool to study white matter injury and subsequent disorders in maturation [29].

Figure 2:

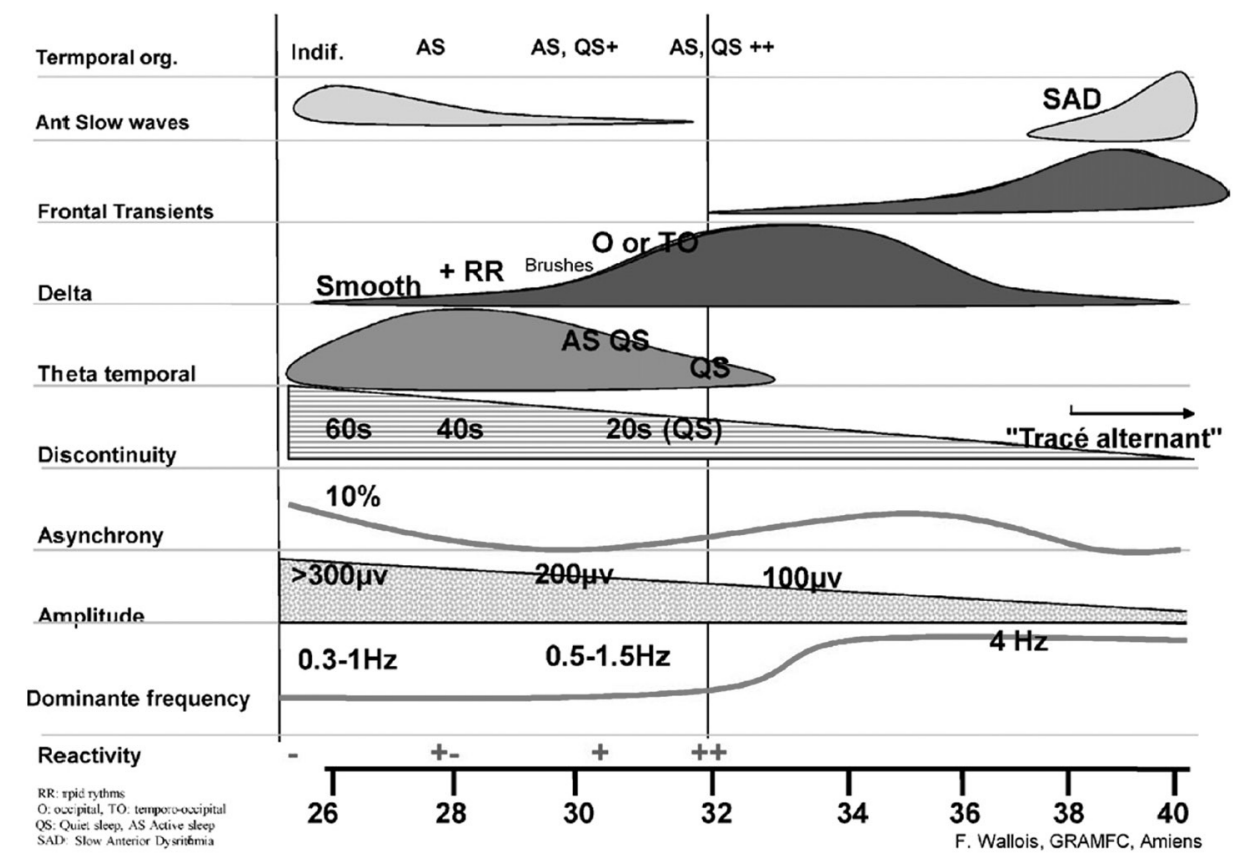

Figure 2 modified from [36] Synopsis of maturation of specific features in EEG of premature neonates.

X-Axis: Postmenstrual Age (weeks) RR: Rapid Rhythm O: Mainly in occipital area TO: Mainly in temporo-occipital area QS: Quiet sleep AS: Acitive sleep SAD: Slow Anterior Dysrythmia

\section{Electroencephalogram}

The conventional electroencephalogram (EEG) provides a direct insight in cerebral function. By using EEG the electrical activity along the scalp, produced by the firing neurons within the brain, is recorded. This electrical activity is caused by the post-synaptic potentials of the cortical neurons [12].

Electrographic recordings of newborns have been performed for more than half a century [30]. EEG patterns of preterm infants change with postmenstrual age (PMA) and have been studied extensively. The maturational changes are discussed in detail in chapter 2. In summary, the preterm 
EEG shows a shift from discontinuous background pattern, which contains segments of short EEG activity (bursts) alternated with segments of EEG inactivity (interburst intervals or $\mathrm{IBI}$ ), towards continuous background pattern, which exists of continuous EEG activity. Also, a decrease in EEG amplitude and an increase in EEG frequency can be observed. The level of synchrony, defined as simultaneous EEG activity in homologous regions of each hemisphere, varies with PMA. Besides, age specific waveforms, like anterior slow waves, frontal transients, delta brushes and temporal theta waves can be observed at different PMA's [31-36].

Moreover, as the preterm infant matures, a distinct sleep-wake pattern develops, which also can be discerned in the EEG. Genuine sleep organization can only be detected at a PMA of 36 weeks[37]. At this age, shifts in sleep states are characterized by changes in EEG background pattern combined with other physiologic behaviors like rapid eye movements (REMs), cardio-respiratory patterns and infant motility patterns. However, rudimentary sleep states are already present in preterm infants with PMA<30 weeks and are characterized by EEG discontinuity and REMs [38, 39]. A synopsis of maturation of specific phemenological features in preterm infants is shown in figure 2.

EEG patterns which run behind actual PMA are called dysmature [33]. Dysmature EEG patterns have been related to adverse outcome later in life [40]. Hayakawa et al. have made a distinction between "disorganized" patterns, following an acute brain insult and related to white matter injury and motor outcome and "dysmature" patterns, reflecting deviations in neuro-development and related to grey matter injury and cognitive outcome [41-44]. Although this distinction of EEG abnormalities is rather resolute and arbitrary, it emphasizes the importance of studying EEG maturation.

However, most studies on EEG maturation were performed in the "pre-digital" era and made use of qualitative visual inspection by EEG experts. This process is very time consuming and may lead to inter-observer variability. The modern digital EEG recorders provide the opportunity to analyze the EEG by automated analysis. In fact, automated EEG quantification techniques are used by anesthesiologists to monitor brain activity in sedated patient $[45,46]$. Besides, automated EEG analysis is already often performed in healthy and asphyxiated full term infants [3, 47-49]. In order to monitor neurophysiologic maturation by more objective and uniform means and make the EEG more accessible to neonatologists, automated quantitative EEG analysis of preterm infants may be valuable.

\section{Neurophysiology}

Although the exact etiology of the EEG signals is yet to be elucidated, some EEG phenomena have been explained [50]. EEG bursts, which are classically described as EEG waves with high amplitude and low frequency between $0.5-4 \mathrm{~Hz}$ [35], are based on spontaneous infra-slow (0.1-0.5 Hz) nonoscillatory activity transients (SATs), which can only be detected by special Direct Current (DC) EEG recorders. The excitatory function of $\gamma$-aminobutyric acid (GABA) at low PMA (see figure 1) may play a permissive role in the generation of SATs. Ongoing (continuous) oscillatory activity is generated cortically and requires activation by cortico-cortical and thalamo-cortical connections. 
The maturation of classical inhibitory action of GABA as well as the development of the GABAergic inter-neuronal network is paralleled by the emergence of continuous activity.

SATs (and therefore bursts) are different from ongoing cortical activity as they are mainly seen in the early developmental period, are self organized, spatially and temporally distinct and can be generated in the cortex in isolation. However, they can also be modulated peripheral input from the thalamo-cortical connections. Indeed, increased SAT activity is seen in the primary sensory cortices when the thalamo-cortical connections are formed in the subplate (around 30 weeks PMA). This thalamo-cortical input may lead to the synchrony in appearance of EEG bursts in both hemispheres. This phenomenon is described by Steriade as "thalamic driving" [51]. Beyond 30 weeks input of developing thalamo-cortical connection disappears and asynchrony of bursts increases. The establishment of (callosal) connections between the two hemispheres leads to an increase in synchrony in bursts from 35 weeks PMA onward.

Simplified, bursts are caused by spontaneous activity whether or not influenced by input of developing thalamo-cortical connections while continuous activity is based on oscillations in a cortico- and thalamo-cortical network. SAT's decrease in amplitude with increasing PMA, which is probably caused by the appearance of gyration, leading to a dispersion of the orientation of cortical electric fields and/or by the increase in the special extent of SAT's (i.e. synchrony), leading to decreasing voltage gradients between adjacent electrodes [50].

\section{Amplitude-integrated EEG}

The amplitude-integrated EEG (aEEG) is a technique which is frequently used to monitor bedside EEG activity in full-term asphyxiated infants [52]. The aEEG monitor records a raw EEG signal usually from one or two channels. The raw EEG signal is amplified and band-passed through an asymmetric filter that strongly attenuates frequencies below 2 and above $15 \mathrm{~Hz}$ in order to minimize artifacts. Hereafter, semi-logarithmic amplitude compression, rectification and time compression takes place. The signal is shown on a screen with on the $x$-axis time $(6 \mathrm{~cm} / \mathrm{h})$ and $y$-axis amplitude (semi-logarithmic; 1-10 $\mu \mathrm{V}$ linear and 10-100 $\mu \mathrm{V}$ logarithmic) [53, 54]. Every 15 seconds a vertical line is drawn with the lower border or lower margin amplitude (LMA) reflecting the minimum peak to peak EEG amplitude and with upper border or upper margin amplitude (UMA) reflecting the maximum peak to peak amplitude (figure 3 ). The bandwidth (BW) reflects the difference between UMA and LMA.

By using the aEEG monitor in full term infants, the neonatologist is informed about the EEG background and presence of convulsions. In fact, the aEEG can be considered as a useful example of automated EEG analysis, which makes the EEG more accessible to non-EEG-expert neonatologist. However, the interpretation of the aEEG recording still relies on visual interpretation which may lead to inter-observer variability. Besides, different classification systems for aEEG background pattern are used $[53,55]$. Also, the visual detection of convulsions still turns out to be a complex task, especially for neonatologists with less experience with aEEG and neonatal seizures may be missed on aEEG [56].

In an effort to overcome this problem we developed an automated aEEG convulsion detection 
algorithm [57]. In most recordings the algorithm reached a sensitivity $\geq 90 \%$. In other recordings the algorithm performed worse, which was mostly due to low agreement between the observers who visually analyzed the aEEG, which served as the gold standard. This illustrates the usefulness of automated analysis of the aEEG in the future.

The aEEG is increasingly being used in preterm infants [58]. Like the raw EEG, the aEEG pattern shows maturational changes. To evaluate aEEG maturation, Burdjalov et al. [59] developed a scoring system which contained different visual established quantitative and non-quantitative aEEG criteria: continuity (defined as overall density of the tracing), lower border amplitude, presence of

Figure 3: Representation of an amplitude-integrated EEG signal in a full term infant

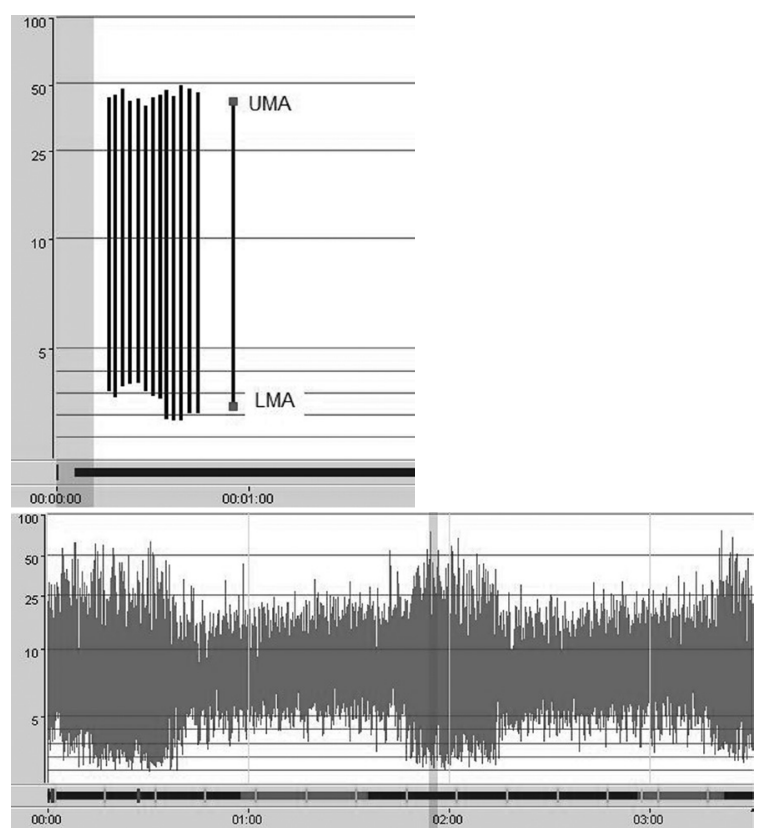

Figure 3, Upper panel: Representation of the amplitude-integrated EEG signal. Every 15 seconds a horizontal line is drawn of which the lower border (Lower Margin Amplitude = LMA) reflects the minimum EEG activity and the upper border (Upper Margin Amplitude $=$ UMA) reflects the maximum activity. Bandwidth (BW) is represented by the black line and expressed as the difference between UMA and LMA.

Lower panel: Amplitude-integrated EEG signal in a full term infant. X-axis represents time $(6 \mathrm{~cm} / \mathrm{hour})$

$\mathrm{Y}$-axis represent amplitude (expressed in $\mu \mathrm{V}$; note the semi-logarithmic scale). Note the cyclical variation in BW which represents the sleep-wake cycle.

sleep wake cycling and bandwidth of tracing (table $1+$ figure 4). Sisman et al. [6o] evaluated aEEG maturation by similar aspects. However, he also assessed upper base voltage (defined as the most common amplitude of the lower margin during high activity and is associated with active sleep or wakefulness), and upper high voltage (defined as the maximum level of activity or the upper edge of the tracing and is associated with quiet sleep). Upper base voltage increased and upper high voltage decreased with PMA. Moreover, data from this study suggest that the extra-uterine 
Table 1: Visual scoring system for amplitude-integrated EEG (aEEG) maturation by Burdjalov [59].

\begin{tabular}{|c|c|c|c|c|}
\hline Score & Continuity & Cycling & $\begin{array}{l}\text { Amplitude of } \\
\text { Lower Border }\end{array}$ & Bandwidth Span and Amplitude of Lower Border \\
\hline 0 & Discontinuous & None & $\begin{array}{l}\text { Severely depressed } \\
(<3 \mu \mathrm{V})\end{array}$ & $\begin{array}{l}\text { Very depressed: low span }(\leq 15 \mu \mathrm{V}) \text { and low voltage } \\
(5 \mu \mathrm{V})\end{array}$ \\
\hline 1 & $\begin{array}{l}\text { Somewhat } \\
\text { continuous }\end{array}$ & Waves first appear & $\begin{array}{l}\text { Somewhat depressed } \\
(3-5 \mu \mathrm{V})\end{array}$ & $\begin{array}{l}\text { Very immature: high span }(>20 \mu \mathrm{V}) \text { or moderate } \\
\text { span }(15-20 \mu \mathrm{V}) \text { and low voltage }(5 \mu \mathrm{V})\end{array}$ \\
\hline 2 & Continuous & $\begin{array}{l}\text { Not definite, } \\
\text { somewhat cycling }\end{array}$ & Elevated $(>5 \mu \mathrm{V})$ & $\begin{array}{l}\text { Immature: high span }(>20 \mu \mathrm{V}) \text { and high voltage } \\
(>5 \mu \mathrm{V})\end{array}$ \\
\hline 3 & & $\begin{array}{l}\text { Definite cycling, but } \\
\text { interrupted }\end{array}$ & & $\begin{array}{l}\text { Maturing: moderate span }(15-20 \mu \mathrm{V}) \text { and high voltage } \\
(>5 \mu \mathrm{V})\end{array}$ \\
\hline 4 & & $\begin{array}{l}\text { Definite cycling, } \\
\text { noninterrupted }\end{array}$ & & Mature: low span $(<15 \mu \mathrm{V})$ and high voltage $(>5 \mu \mathrm{V})$ \\
\hline 5 & & $\begin{array}{l}\text { Regular and mature } \\
\text { cycling }\end{array}$ & & \\
\hline
\end{tabular}

Figure 4: Progressive series of amplitude-integrated EEG (aEEG) recordings of a preterm infant with component score values.
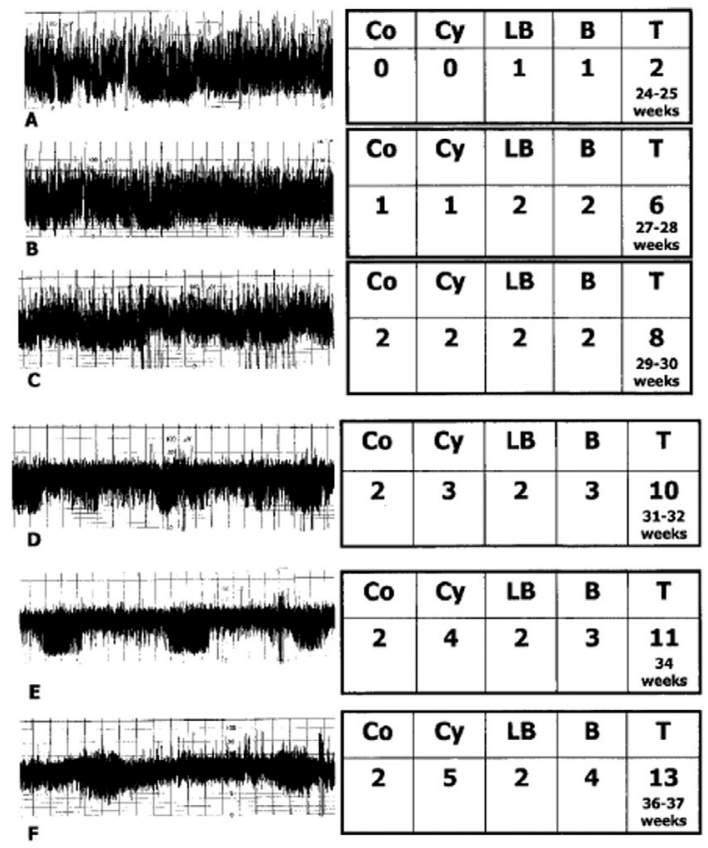

Figure 4 modified from [59] Shows progressive amplitude-integrated EEG (aEEG) recordings of a preterm infant with score values of the different components of the scoring system proposed by Burdjalov to assess aEEG maturation. Co: Continuity of the recording Cy: Cyclicity LB: Lower border amplitude score B: Bandwidth T: Total score. The actual post menstrual age in weeks is also represented.

environment accelerates aEEG maturation. Olischar et al. [61] assessed the aEEG of preterm infants by other quantitative criteria (table 2). An increase of the continuous aEEG background pattern with PMA was observed. Kuint et al. [62] analyzed the aEEG characteristics during quiet sleep, when the aEEG bandwidth broadens, and during active sleep or awake state, when the aEEG bandwidth is at the most common value. 
Table 2: Quantitative maturational amplitude-integrated EEG (aEEG) characteristics of preterm infants.

\begin{tabular}{|c|c|c|c|}
\hline Burdjalov[59] & Olischar[61] & Sisman[60] & Kuint[62] \\
\hline $\begin{array}{l}\text { Very depressed: } \\
\text { BW }<15 \mu \mathrm{V} \\
\mathrm{LMA}<5 \mu \mathrm{V}\end{array}$ & $\begin{array}{l}\text { Discontinuous low } \\
\text { voltage: } \\
\text { LMA }<3 \mu \mathrm{V} \\
\text { UMA } 15-30 \mu \mathrm{V}\end{array}$ & $\begin{array}{l}\text { Common UMA (during } \\
\text { AS or AW): } \\
\text { Increasing with PMA }\end{array}$ & $\begin{array}{l}\text { BW during AS: } \\
\text { decrease with PMA }\end{array}$ \\
\hline $\begin{array}{l}\text { Very immature: } \\
\text { BW }>20 \mu \mathrm{V} \\
\text { LMA } 5 \mu \mathrm{V}\end{array}$ & $\begin{array}{l}\text { Discontinuous high } \\
\text { voltage: } \\
\text { LMA 3-5 } \mu \mathrm{V} \\
\text { UMA 20-40 } \mu \mathrm{V}\end{array}$ & $\begin{array}{l}\text { Highest UMA (during } \\
\text { QS): } \\
\text { Decreasing with PMA }\end{array}$ & $\begin{array}{l}\text { BW during QS: } \\
\text { no change }\end{array}$ \\
\hline $\begin{array}{l}\text { Immature: } \\
\text { BW }>20 \mu \mathrm{V} \\
\text { LMA }>5 \mu \mathrm{V}\end{array}$ & $\begin{array}{l}\text { Continuous: } \\
\text { LMA }>5 \mu \mathrm{V} \\
\text { UMA } 20-40 \mu \mathrm{V}\end{array}$ & $\begin{array}{l}\text { LMA: } \\
\text { Increasing with PMA }\end{array}$ & $\begin{array}{l}\text { LMA during: AS: } \\
\text { increase with PMA }\end{array}$ \\
\hline $\begin{array}{l}\text { Maturing: } \\
\text { BW 15-20 } \mu \mathrm{V} \\
\text { LMA > } 5 \mu \mathrm{V}\end{array}$ & & $\begin{array}{l}\text { BW: } \\
\text { Decreasing with PMA }\end{array}$ & $\begin{array}{l}\text { LMA during QS: } \\
\text { no change }\end{array}$ \\
\hline $\begin{array}{l}\text { Mature: } \\
\text { BW }<15 \mu \mathrm{V} \\
\text { LMA }>5 \mu \mathrm{V}\end{array}$ & & & \\
\hline $\begin{array}{l}\text { The different quanti } \\
\text { Note that in these s } \\
\text { BW: Bandwidth LM } \\
\text { Sleep PMA: Postmer } \\
\text { During Quiet sleep } \\
\text { has a smaller value }\end{array}$ & $\begin{array}{l}\text { acteristics, which were us } \\
\text { parameters have been as } \\
\text { argin amplitude UMA: U } \\
\text { andwidth is broadens wh } \\
\text { gure 5). }\end{array}$ & $\begin{array}{l}\text { ssess the maturation of the a } \\
\text { I by visual analysis only. } \\
\text { argin Amplitude AS: Active } \\
\text { ing Active Sleep and Awake }\end{array}$ & $\begin{array}{l}\text { plitude-integrated EEG (aEEG). } \\
\text { ep AW: Awake State QS: Quiet } \\
\text { te the bandwidth of the aEEG }\end{array}$ \\
\hline
\end{tabular}

Table 3: The suitability of the Electroencephalogram (EEG) and the amplitude-integrated electroencephalogram (aEEG) for clinical use at the NICU.

\begin{tabular}{|c|c|c|}
\hline Aspect & EEG & aEEG \\
\hline Simple to record & 0 & + \\
\hline Feasible to evaluate for non-expert & 0 & + \\
\hline Detection of seizures & ++ & + \\
\hline Risk of artifacts influencing interpretation & 0 & - \\
\hline Useful for long-term bed-side monitoring & 0 & + \\
\hline Precision of information & + & 0 \\
\hline Information about sleep-wake state & ++ & + \\
\hline
\end{tabular}




\section{Aim of the thesis}

As discussed above, both the EEG and aEEG signal show maturational changes (figure 5). As the EEG and aEEG technique are complementary to each other in clinical use (table 3), each individual technique is interesting to study in respect to electro-cortical maturation. However, both techniques rely on visual interpretation.

As stated before we aim to investigate which conditions interfere with brain development of preterm infants. This can only be carried out when brain development of "healthy" preterm infants is accurately documented.

Figure 5: Two examples illustrate differences in EEG and aEEG at different PMA.
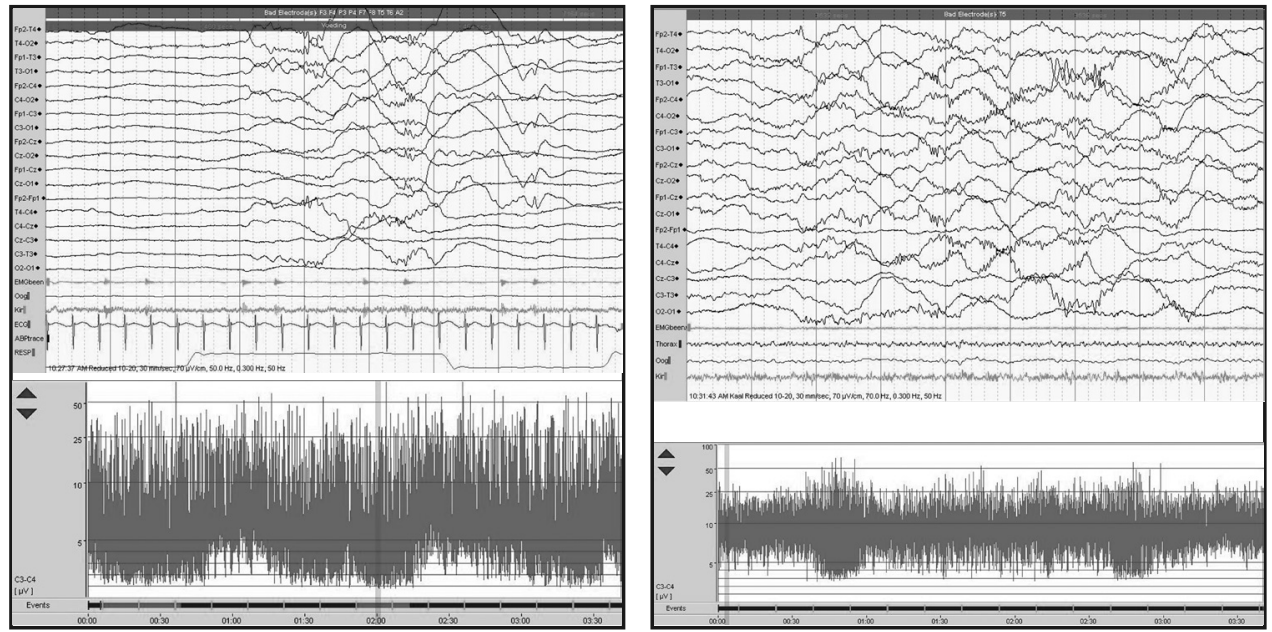

The electroencephalogram (EEG; upper side) and amplitude-integrated EEG (aEEG, bottom side) of one infant, who was enrolled in our study. Y-axis represents voltage $(\mu \mathrm{V})$; $x$-axis represents time: $E E G(3 \mathrm{~cm} / \mathrm{s})$ aEEG $(6 \mathrm{~cm} / \mathrm{h})$.

EEG and aEEG were recorded at a postmenstrual age of $27+4$ weeks and $33+4$ weeks, respectively. The left EEG consists of a discontinuous pattern, which contains periods of EEG inactivity (interburst) alternated with bursts of high amplitude, low frequent EEG activity. The corresponding amplitude-integrated EEG below shows a discontinuous voltage pattern. A distinct aEEG sleep wake cycle cannot be observed yet. The right EEG shows a continuous pattern, which exist of continuous EEG activity. Note that the amplitude of EEG activity is lower, and frequency of EEG is higher in the right EEG. Also an age specific waveform is present: the temporal sawtooth. The corresponding aEEG shows a continuous voltage. Sleep wake cycling can be recognized by repeated periods of broader aEEG bandwidth during quiet sleep.

To investigate the feasibility of automated analysis of electro-cortical maturation in healthy preterm infants, we started a prospective study in the Máxima Medical Centre Veldhoven. In this study, weekly EEG recordings were performed in a strictly selected population of healthy preterm infants. The obtained data were analyzed by automated analysis in collaboration with clinical physicists of Máxima Medical Centre and in collaboration with the department of Applied Physics of the Eindhoven Technical University. 
This study has two main questions:

1. Is it feasible to assess neurophysiologic maturation of "healthy" preterm infants with automated analysis of the EEG and aEEG, respectively?

2. Which of the computer-assisted electrocortical parameters are valuable to describe neurophysiologic maturation of "healthy" preterm infants?

\section{Outline}

In chapter $\mathbf{2}$ we review the maturation of the EEG in preterm infant and discuss which EEG aspects are suitable for quantitative automated analysis. In chapter 3 , we investigate the maturational changes in EEG discontinuity in a cohort of healthy preterm infants with use of several already existing automated algorithms. In chapter 4, we aim to develop a simple method for automated detection of burst, intervals and continuous patterns in order to analyse maturational changes in EEG discontinuity more precisely. In chapter 5, we investigate if neurophysiologic maturation in very preterm infants can be described by automated spectral power analysis of whole 4-h EEG recordings and to try to identify specific changes of spectral power measures with maturation. In chapter 6, we combine automated burst detection and spectral analysis in order to quantify the EEG burst frequency spectrum of preterm infants and to decribe the topography of maturational changes in spectral parameters of bursts.

In chapter 7, we study the maturational changes in the single channel amplitude-integrated EEG (aEEG) in a cohort of healthy preterm infants by automated analysis. In chapter $\mathbf{8}$, we investigate maturational characteristics of multi-channel aEEG by automated analysis and asses regional differences. In chapter $\mathbf{9}$, we discuss the results of this study project and contemplate on future perspectives.

\section{Methods}

\section{Patient recruitment}

The following patients were eligible for inclusion at first glance: gestational age < 32 weeks; Apgar score $\geq 6$ at 5 minutes; appropriate-for-gestational age, birth weight $>10^{\text {th }}$ percentile; arterial umbilical $\mathrm{pH} \geq 7.00$; normal cerebral ultrasounds (intraventricular hemorrhage $\leq \mathrm{I}$, no congenital malformations) in first week; and expected stay at our hospital > 2 weeks. Note: for one study (chapter 8) infants with a gestational age between 32-37 weeks were selected, in these infants only one EEG recording was performed at the end of the first week of life.

The following criteria were used to exclude infants: hemodynamic instability (requiring volume expansion and/or inotropics; abnormalities on cerebral ultrasound (intraventricular hemorrhage $\geq \mathrm{II}$, periventricular leucomalacia) [10, 65]; neuro-infection; use of sedative and/or anti-epileptic medication within $48 \mathrm{~h}$ of recording; doxapram use; no parental consent. Excluded from analyses were infants with development of bronchopulmonary dysplasia (defined as the use of supplemental oxygen or respiratory support at 36 weeks PMA) [66] or developmental delay (Bayley Infant 
Table 4: Frequency bands of the EEG

\section{Frequency}

\begin{tabular}{ll}
\hline Delta-1 & $0.5-1 \mathrm{~Hz}$ \\
\hline Delta-2 & $1-4 \mathrm{~Hz}$ \\
\hline Theta & $4-8 \mathrm{~Hz}$ \\
\hline Alpha & $8-13 \mathrm{~Hz}$ \\
Beta & $13-30 \mathrm{~Hz}$ \\
\hline \hline
\end{tabular}

Figure 6[70]: Electrodes placed according to the 10-20 reduced montage system.

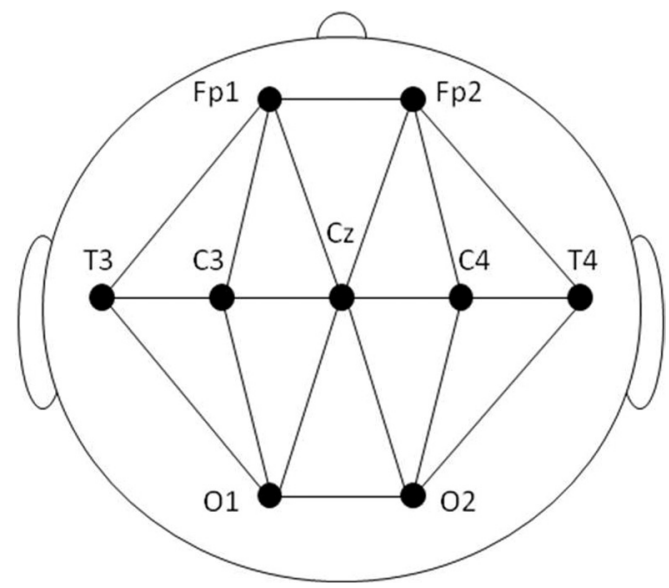

Development scores $<85$ at 1 or 2 years of corrected age) $[67,68]$.

Of the patients who were included in the study, the following data were noted: delivery modus, maternal corticosteroid and medication administration, gestational age, sex, birth weight, Apgar score, arterial umbilical pH, CRIB score [69], medication, grade of respiratory distress syndrome based on chest X-ray, respiratory support, serum blood glucose levels (lowest, highest and at recording), carbon dioxide levels in blood gasses (lowest and highest), highest total serum bilirubine levels, highest C-reactive protein, caffeine level, presence of sepsis (positive blood culture) and result from cranial ultrasounds.

The studied population was followed up in our perinatal centre at 6, 12 and 24 months of corrected age. The patients were assessed with the Bayley Scales of Infant Development II or III for mental and motor function $[67,68]$. 


\section{Ethical considerations and parental consent}

This study was approved by the local medical ethical committee (METC Veldhoven, registration number 2006-0613). For the inclusion of older infants an amendment was proposed (2007-0613R) and also approved. Studies started after (written) parental consent was obtained.

\section{Recordings}

Starting at the end of the first week of life, weekly 24-channel digital EEG recordings (NicoletOne ${ }^{\oplus}$; Viasys Healthcare, Conshohocken, $\mathrm{Pa}$, USA) were performed. The EEG recordings started at $8 \mathrm{AM}$ with a recording time of 4 hours. The infant was in prone or side position during the recording.

After skin preparation (Nuprep Gel; D.O. Weaver Co., Aurora, Colo., USA), Ag/AgCl cup electrodes, filled with a conductive paste (Ten2o; D.O. Weaver Co.), were placed according to the international 10-20 reduced montage system for newborns [70] (figure 6).

The following recording settings were used: Low pass filter $30 \mathrm{~Hz}$. High pass filter $0.3 \mathrm{~Hz}$. Notch filter: $50 \mathrm{~Hz}$.Sensitivity $7 \mu \mathrm{V} / \mathrm{mm}$. Paper speed $30 \mathrm{~mm} / \mathrm{s}$. Sample frequency $512 \mathrm{~Hz}$.

\section{Analysis of data}

The maturational aspects of the infant EEG which are promising for quantitative analysis are discussed in chapter 2 . In summary, the following aspects were analyzed:

a. EEG aspects

Background continuity

Length of continuous, discontinuous tracings

Length of interbursts intervals and bursts

Overall discontinuity of the EEG trace

Changes in EEG frequency and amplitude (table 4)

Spectral analysis of total EEG tracing

Spectral analysis of bursts

Regional differences in spectral frequencies

b. amplitude-integrated EEG aspects

Change in continuity (Lower margin amplitude)

Change in amplitude (Upper margin amplitude)

Development of sleep wake cycling (Bandwidth) 
1. Bayless S, Stevenson J. Executive functions in school-age children born very prematurely. Early Hum Dev 2007;83(4):247-54.

2. Cooke RW. Preterm mortality and morbidity over 25 years. Arch Dis Child Fetal Neonatal Ed 2006;91(4):F293-4.

3. Larroque B, Ancel PY, Marret S, Marchand L, Andre M, Arnaud C, et al. Neurodevelopmental disabilities and special care of 5-year-old children born before 33 weeks of gestation (the EPIPAGE study): a longitudinal cohort study. Lancet 2008;371(9615):813-20.

4. Gressens $P$, Rogido $M$, Paindaveine $B$, Sola A. The impact of neonatal intensive care practices on the developing brain. J Pediatr 2002;140(6):646-53.

5. Perlman JM. Cognitive and behavioral deficits in premature graduates of intensive care. Clin Perinatol 2002;29(4):779-97.

6. Volpe J. Brain injury in premature infants: a complex amalgam of destructive and developmental disturbances. Lancet Neurol 2009;8(1):110-24.

7. Kostovic I, Jovanov-Milosevic N. The development of cerebral connections during the first 20-45 weeks' gestation. Semin Fetal Neonatal Med 2006;11(6):415-22.

8. Marin-Padilla M. Dual origin of the mammalian neocortex and evolution of the cortical plate. Anat Embryol (Berl) 1978;152(2):109-26.

9. Rakic P. Neuronal migration and contact guidance in the primate telencephalon. Postgrad Med J 1978;54 Suppl 1:25-40.

10. Volpe JJ. Neurology of the newborn. 5th Edition. Philadelphia: Elsevier; 2008.

11. de Graaf-Peters VB, Hadders-Algra M. Ontogeny of the human central nervous system: what is happening when? Early Hum Dev 2006;82(4):257-66.

12. Mento G, Bisiacchi PS. Neurocognitive development in preterm infants: Insights from different approaches. Neurosci Biobehav Rev 2011.

13. Kostovic I, Judas M. The development of the subplate and thalamocortical connections in the human foetal brain. Acta Paediatr 2010;99(8):1119-27.

14. Volpe JJ. The encephalopathy of prematurity-brain injury and impaired brain development inextricably intertwined. Semin Pediatr Neurol 2009;16(4):167-78.

15. Dubois J, Benders M, Cachia A, Lazeyras F, Ha-Vinh Leuchter R, Sizonenko SV, et al. Mapping the early cortical folding process in the preterm newborn brain. Cereb Cortex 2008;18(6):1444-54.

16. Mewes $A U$, Huppi PS, Als H, Rybicki FJ, Inder $T E$, McAnulty GB, et al. Regional brain development in serial magnetic resonance imaging of low-risk preterm infants. Pediatrics 2006;118(1):23-33.

17. Inder TE, Warfield SK, Wang H, Huppi PS, Volpe JJ. Abnormal cerebral structure is present at term in premature infants. Pediatrics 2005; 115(2):286-94.

18. Constable RT, Ment $L R$, Vohr BR, Kesler $S R$, Fulbright $R K$, Lacadie $C$, et al. Prematurely born children demonstrate white matter microstructural differences at 12 years of age, relative to term control subjects: an investigation of group and gender effects. Pediatrics 2008;121(2):306-16.

19. Cooke RW, Abernethy LJ. Cranial magnetic resonance imaging and school performance in very low birth weight infants in adolescence. Arch Dis Child Fetal Neonatal Ed 1999;81(2):F116-21.

20. Isaacs EB, Lucas A, Chong WK, Wood SJ, Johnson CL, Marshall C, et al. Hippocampal volume and everyday memory in children of very low 
birth weight. Pediatr Res 2000;47(6):713-20.

21. El-Dib M, Massaro AN, Bulas D, Aly H. Neuroimaging and neurodevelopmental outcome of premature infants. Am J Perinatol 2010;27(10):803-18.

22. Peterson BS, Anderson AW, Ehrenkranz R, Staib $L H$, Tageldin M, Colson E, et al. Regional brain volumes and their later neurodevelopmental correlates in term and preterm infants. Pediatrics 2003;111(5 Pt 1):939-48.

23. Woodward LJ, Anderson PJ, Austin NC, Howard $K$, Inder TE. Neonatal MRI to predict neurodevelopmental outcomes in preterm infants. $N$ Engl J Med 2006;355(7):685-94.

24. Ment LR, Kesler S, Vohr B, Katz KH, Baumgartner $H$, Schneider KC, et al. Longitudinal brain volume changes in preterm and term control subjects during late childhood and adolescence. Pediatrics 2009;123(2):503-11.

25. Als H, Duffy FH, McAnulty GB, Rivkin MJ, Vajapeyam S, Mulkern RV, et al. Early experience alters brain function and structure. Pediatrics 2004;113(4):846-57.

26. Milgrom J, Newnham C, Anderson PJ, Doyle LW, Gemmill AW, Lee K, et al. Early sensitivity training for parents of preterm infants: impact on the developing brain. Pediatr Res 2010;67(3):330-5.

27. Huppi PS, Dubois J. Diffusion tensor imaging of brain development. Semin Fetal Neonatal Med 2006;11(6):489-97.

28. Mathur AM, Neil JJ, Inder TE. Understanding brain injury and neurodevelopmental disabilities in the preterm infant: the evolving role of advanced magnetic resonance imaging. Semin Perinatol 2010;34(1):57-66.

29. Dudink J, Lequin M, van Pul C, Buijs J, Conneman N, van Goudoever J, et al. Fractional anisotropy in white matter tracts of very-low-birth-weight infants. Pediatr Radiol 2007;37(12):1216-23.

30. Samson-Dollfus D. L'electroencephalogramme du premature jusqu'a l'age de trois mois et du nouveau ne a terme. Foulon Paris: These Med 1955.

31. Anderson CM, Torres F, Faoro A. The EEG of the early premature. Electroencephalogr Clin Neurophysiol 1985;60(2):95-105.

32. Andre M, Lamblin MD, d'Allest AM, CurziDascalova L, Moussalli-Salefranque F, TSNT, et al. Electroencephalography in premature and full-term infants. Developmental features and glossary. Neurophysiol Clin 2010;40(2):59-124.

33. Lombroso CT. Neonatal polygraphy in full-term and premature infants: a review of normal and abnormal findings. J Clin Neurophysiol 1985;2(2):105-55.

34. Scher MS. Normal electrographic-polysomnographic patterns in preterm and fullterm infants. Semin Pediatr Neurol 1996;3(1):2-12.

35. Selton D, Andre M, Hascoet JM. Normal EEG in very premature infants: reference criteria. Clin Neurophysiol 2000;111(12):2116-24.

36. Wallois F. Synopsis of maturation of specific features in EEG of premature neonates. Neurophysiol Clin 2010;40(2):125-6.

37. ScherMS. Ontogeny of EEG sleep from neonatal through infancy periods. Handb Clin Neurol 2008;98:111-29.

38. Curzi-Dascalova L, Figueroa JM, Eiselt M, Christova E, Virassamy A, d'Allest AM, et al. Sleep state organization in premature infants of less than 35 weeks' gestational age. Pediatr Res 1993;34(5):624-8.

39. Scher MS, Johnson MW, Holditch-Davis D. Cyclicity of neonatal sleep behaviors at 25 to 30 weeks' postconceptional age. Pediatr Res 2005;57(6):879-82.

40. Tharp BR, Scher MS, Clancy RR. Serial EEGs in normal and abnormal infants with birth weights less than 1200 grams--a prospective study with long term follow-up. Neuropediatrics 1989;20(2):64-72

41. Hayakawa F, Okumura A, Kato T, Kuno K, 
Watanabe K. Dysmature EEG pattern in EEGs of preterm infants with cognitive impairment: maturation arrest caused by prolonged mild CNS depression. Brain Dev 1997;19(2):122-5.

42. Hayakawa F, Okumura A, Kato T, Kuno K, Watanabe K. Disorganized patterns: chronic-stage EEG abnormality of the late neonatal period following severely depressed EEG activities in early preterm infants. Neuropediatrics 1997;28(5):272-5.

43. Okumura A, Hayakawa F, Kato T, Kuno K, Watanabe K. Developmental outcome and types of chronic-stage EEG abnormalities in preterm infants. Dev Med Child Neurol 2002;44(11):729-34.

44. Watanabe K, Hayakawa F, Okumura A. Neonatal EEG: a powerful tool in the assessment of brain damage in preterm infants. Brain Dev 1999;21(6):361-72.

45. Bowdle TA. Depth of anesthesia monitoring. Anesthesiol Clin 2006;24(4):793-822.

46. Powers KS, Nazarian EB, Tapyrik SA, Kohli SM, Yin $H$, van der Jagt EW, et al. Bispectral index as a guide for titration of propofol during procedural sedation among children. Pediatrics 2005;115(6):1666-74.

47. Korotchikova I, Stevenson NJ, Walsh BH, Murray DM, Boylan GB. Quantitative EEG analysis in neonatal hypoxic ischaemic encephalopathy. Clin Neurophysiol 2011.

48. Lofhede J, Lofgren N, Thordstein M, Flisberg A, Kjellmer I, Lindecrantz K. Classification of burst and suppression in the neonatal electroencephalogram. J Neural Eng 2008;5(4):402-10.

49. Lofhede J, Thordstein M, Lofgren N, Flisberg A, Rosa-Zurera M, Kjellmer l, et al. Automatic classification of background EEG activity in healthy and sickneonates. J Neural Eng 2011;7(1):16007.

50. Vanhatalo S, Kaila K. Development of neonatal EEG activity: from phenomenology to physiology. Semin Fetal Neonatal Med 2006;11(6):471-8.

51. Steriade M, Gloor P, Llinas RR, Lopes de Silva
FH, Mesulam MM. Report of IFCN Committee on Basic Mechanisms. Basic mechanisms of cerebral rhythmic activities. Electroencephalogr Clin Neurophysiol 1990;76(6):481-508.

52. Maynard D, Prior PF, Scott DF. Device for continuous monitoring of cerebral activity in resuscitated patients. BrMed J 1969;4(5682):545-6.

53. de Vries LS, Toet MC. Amplitude integrated electroencephalography in the full-term newborn. Clin Perinatol 2006;33(3):619-32, vi.

54. Rosen I. The physiological basis for continuous electroencephalogram monitoring in the neonate. Clin Perinatol 2006;33(3):593-611, v.

55. al Naqeeb N, Edwards AD, Cowan FM, AzzopardiD. Assessment of neonatal encephalopathy by amplitude-integrated electroencephalography. Pediatrics 1999;103(6 Pt 1):1263-71.

56. Shellhaas RA, Soaita Al, Clancy RR. Sensitivity of amplitude-integrated electroencephalography for neonatal seizure detection. Pediatrics 2007;120(4):770-7.

57. Lommen CM, Pasman JW, van Kranen VH, Andriessen P, Cluitmans PJ, van Rooij LG, et al. An algorithm for the automatic detection of seizures in neonatal amplitude-integrated EEG. Acta Paediatr 2007;96(5):674-80.

58. Tao JD, Mathur AM. Using amplitude-integrated EEG in neonatal intensive care. J Perinatol 2010;30 Suppl:S73-81.

59. Burdjalov VF, Baumgart S, Spitzer AR. Cerebral function monitoring: a new scoring system for the evaluation of brain maturation in neonates. Pediatrics 2003;112(4):855-61.

60. Sisman J, Campbell DE, Brion LP. Amplitudeintegrated EEG in preterm infants: maturation of background pattern and amplitude voltage with postmenstrual age and gestational age. J Perinatol 2005;25(6):391-6.

61. Olischar M, Klebermass $K$, Kuhle S, Hulek M, Kohlhauser C, Rucklinger E, et al. Reference values for amplitude-integrated electroencephalographic activity in preterm infants 
younger than 30 weeks' gestational age.

Pediatrics 2004;113(1 Pt 1):e61-6.

62. Kuint J, Turgeman A, Torjman A, MaayanMetzger A. Characteristics of amplitude-integrated electroencephalogram in premature infants. J Child Neurol 2007;22(3):277-81.

63. Suk D, Krauss $A N$, Engel M, Perlman JM. Amplitude-integrated electroencephalography in the NICU: frequent artifacts in premature infants may limit its utility as a monitoring device. Pediatrics 2009;123(2):e328-32.

64. Toet MC, Lemmers PM. Brain monitoring in neonates. Early Hum Dev 2009;85(2):77-84.

65. de Vries $L S$, Eken $P$, Dubowitz LM. The spectrum of leukomalacia using cranial ultrasound. Behav Brain Res 1992;49(1):1-6.

66. Lal MK, Manktelow BN, Draper ES, Field DJ. Chronic lung disease of prematurity and intrauterine growth retardation: a populationbased study. Pediatrics 2003;111(3):483-7.

67. Bayley N. Manual for Bayley Scales of Infant Development. 2nd Edition. San Antonio, Texas: The Psychological Corporation; 1993.

68. Bayley N. Bayley Scales of Infant and Toddler Development. 3rdEdition. San Antonio, Texas: The Psychological Corporation; 2005.

69. The CRIB (clinical risk index for babies) score: $a$ tool for assessing initial neonatal risk and comparing performance of neonatal intensive care units. The International Neonatal Network. Lancet 1993;342(8865):193-8.

70. Tekgul H, Bourgeois BF, Gauvreau K, Bergin AM. Electroencephalography in neonatal seizures: comparison of a reduced and a full 10/20 montage. Pediatr Neurol 2005;32(3):155-61. 


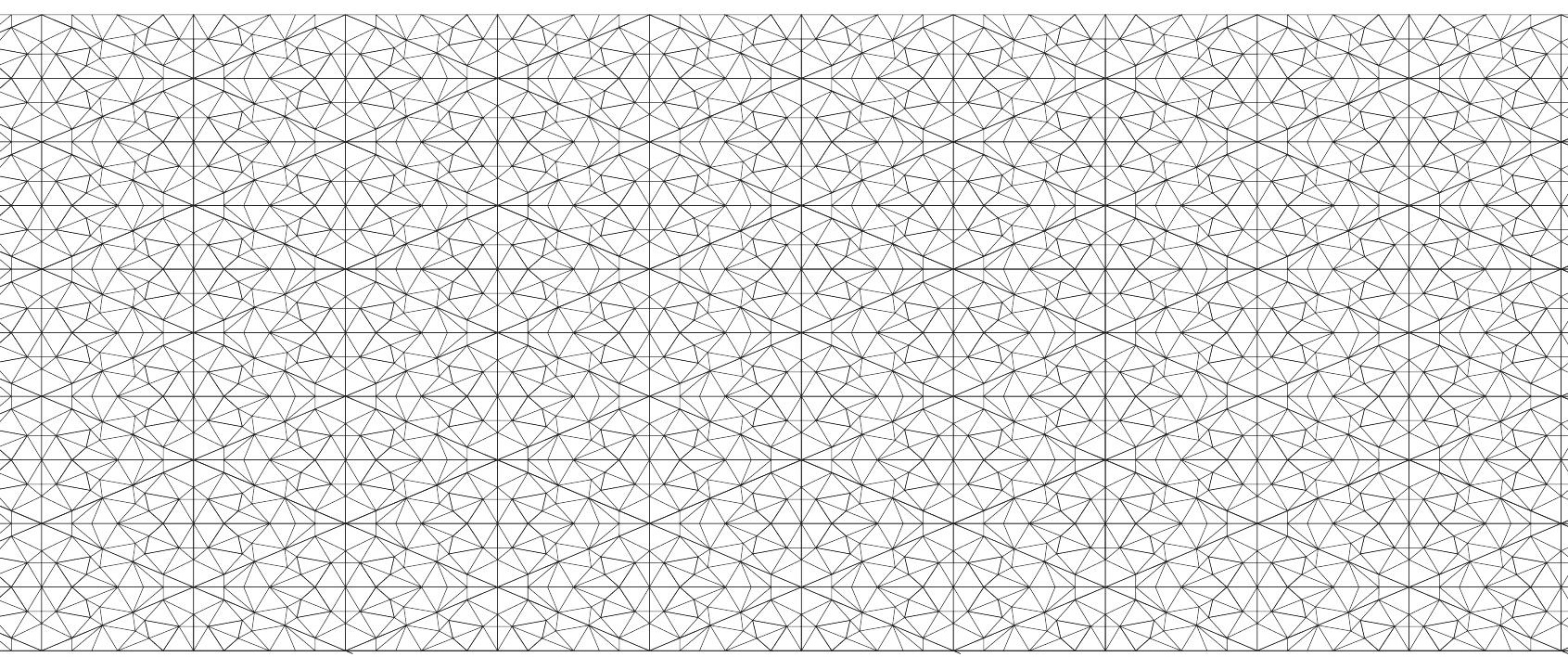




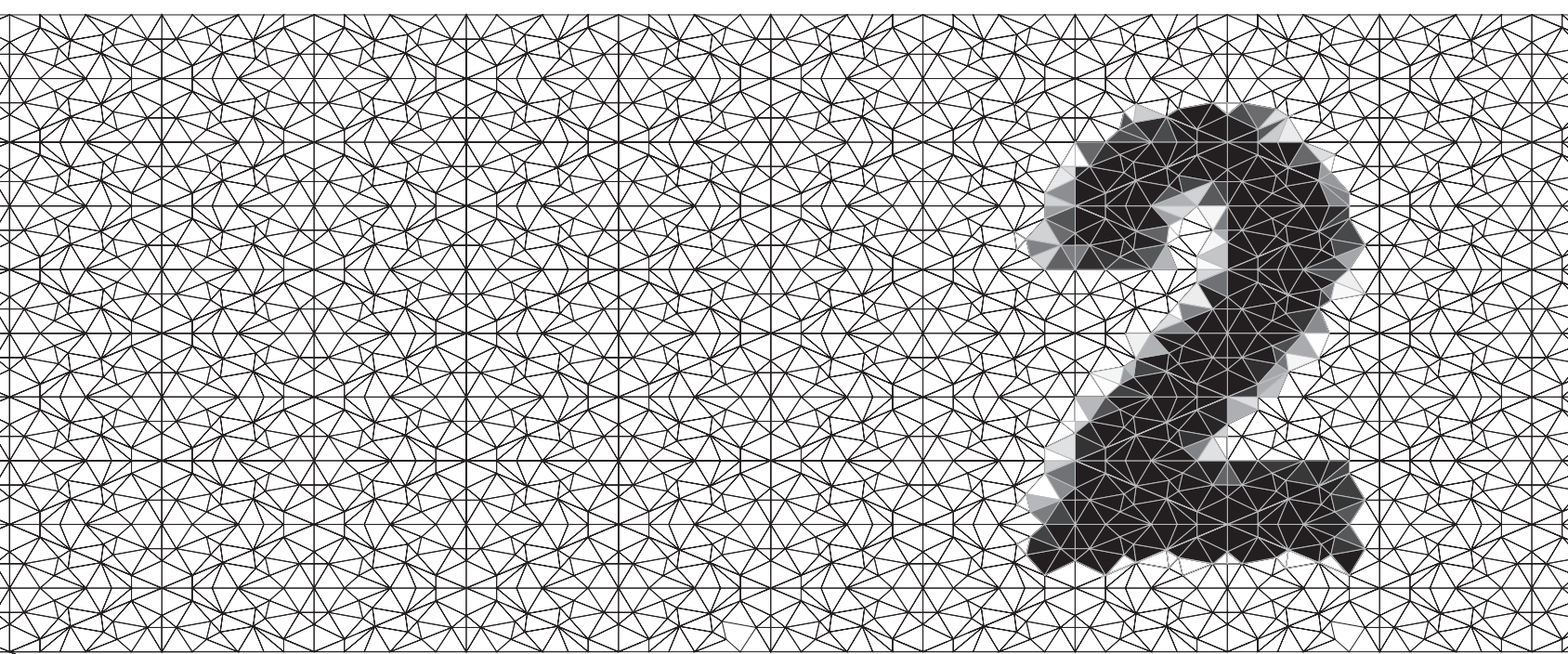

\title{
Analyzing EEG maturation in preterm infants: The value of a quantitative approach
}

\author{
Hendrik J. Niemarkt \\ Peter Andriessen \\ Jaco Pasman \\ Johan S. Vles \\ Luc J. Zimmermann
} Sidarto Bambang Oetomo 


\section{Abstract}

Despite increase in survival of very low birth weight infants, the number of infants who experience neuromotor or neurocognitive problems later in life is still high.

Therefore, accurate documentation of the brain development in these infants is indicated. Electroencephalography (EEG) may be a valuable tool to monitor brain development during the intensive care period. However, our knowledge on EEG maturation is based on visually determined patterns. Since there are no exact reference values, conclusions on EEG maturation are difficult to establish and may vary between investigators. Also, most knowledge concerning EEG maturation is derived from relatively old studies in the "pre-digital" era.

New digital EEG recorders provide the opportunity towards a quantitative approach of EEG maturation. This approach may lead to a more objective and uniform description of EEG maturation. In this article we review the most important aspects of EEG maturation, i.e. changes in discontinuity, development of age specific waveforms, inter-hemispheric synchrony/correlation and the development of sleep-wake cycles, and qualitative or semi-quantitative studies concerning EEG maturation and outcome.

We discuss the concept of an EEG analyzing algorithm and propose several quantitative analyzing techniques which may be incorporated in such an algorithm. 


\section{Introduction}

Advances in neonatal intensive care for very low birth weight infants have increased the survival of these infants [1, 2]. However, the number of infants who experience neuromotor and neurocognitive deficits later in life is still high, even in the absence of neurosonographic abnormalities [3-6]. Follow up studies indicate that $5-15 \%$ of these infants develop cerebral palsy and $25-50 \%$ experience abnormalities in cognition and behavior, with learning disturbances nearly uniformly as result [7]. The exact etiology of these neurodevelopmental deficits remains to be clarified, but it is suggested that different medical, iatrogenic and environmental conditions in the neonatal intensive care unit may interfere with the development of the vulnerable premature brain $[6,8,9]$.

Conventional electroencephalography (EEG) may be regarded as the gold standard in the assessment of cerebral function. Typical neonatal EEG patterns change as a function of gestational age (GA) and postmenstrual age (PMA, GA + postnatal age). Therefore, EEG may be a valuable tool to monitor brain developmentin these infants. In preterm infants, electrical maturity can be estimated within \pm 2 weeks PMA by visual inspection only $[10,11]$. In fact, estimation of PMA is suggested to be more difficult in younger infants because of wider ranges in normal values [12]. So, one may not be able to detect maturational changes in (bi-)weekly EEG recordings as these are taken within the measurement error.

However, a great part of knowledge concerning EEG maturation in the preterm is derived from the "predigital" era when methodological issues, which were associated to the pre-digital recordings, may contribute to the low accuracy in the estimation of PMA. Examples of these issues are the fixed montage, filtering and sensitivity of EEG recordings. Also, it takes an expert on neonatal EEG with plenty of time to visually analyze a preterm EEG on its maturational characteristics. And even in that case, this expert may come to other conclusions than another expert, as definitions of maturational aspects of the preterm EEG and method of analyzing (i.e. choice of montage, filtering and sensitivity) are not uniform.

In the field of anesthesiology, automated quantification techniques of EEG have been used to monitor brain activity in sedated patients $[13,14]$. These techniques create the possibility for EEG non-experts to evaluate sedation directly and by uniform manners.

In order to describe neurophysiologic maturation more precisely and by objective and uniform means, a quantitative approach towards the neonatal EEG also may be preferable. New digital EEG recording devices, creating the possibility for filtering and processing the raw EEG on- and off-line, provide an unique opportunity for this approach.

In this article we review the most important aspects of EEG maturation in preterm infants. We discuss the concept of an EEG analyzing algorithm and propose several quantitative analyzing techniques which may be incorporated in such an algorithm.

\section{Changes in continuity}

The EEG of the very preterm infant ( $<32$ weeks PMA) consists of two complementary background patterns. First, a continuous pattern or tracé continue,which consist of continuous EEG activity 
above a certain cerebral electrical threshold value. Second, a discontinuous pattern, which consists of bursts of EEG activity alternated with periods of EEG quiescence, named interburst interval (IBI) (figure 1) [10, 15-17]. The discontinuous pattern can be subdivided in two patterns, namely the tracé discontinue and the tracé alternant, determined by a visually interpretation of the amplitude of the EEG signal. The tracé alternant pattern is not observed until 34 weeks GA [18].

The time percentage of continuous and discontinuous tracings was studied by several authors (table 1). All authors found an increase in time percentage of continuous tracings and decrease in time percentage of discontinuous tracings, however with great variability in and between studies. This may be caused by the use of qualitative or semi-quantitative definitions of the discontinuous and continuous background patterns.The continuous and discontinuous background patterns are physiologic at low PMA. To our knowledge, there are no studies that observed a correlation between time percentage of (dis)continuous tracings at low PMA and cerebral pathology and adverse outcome later in life. However, the discontinuous pattern must be distinguished from the burst-suppression (BS) pattern. The BS pattern also consists of alternating periods of EEG activity and quiescence but shows fixed burst periods between 1-10 s, absence of activity or very low activity

Figure 1: An example of an EEG in an infant with PMA of 30 weeks.
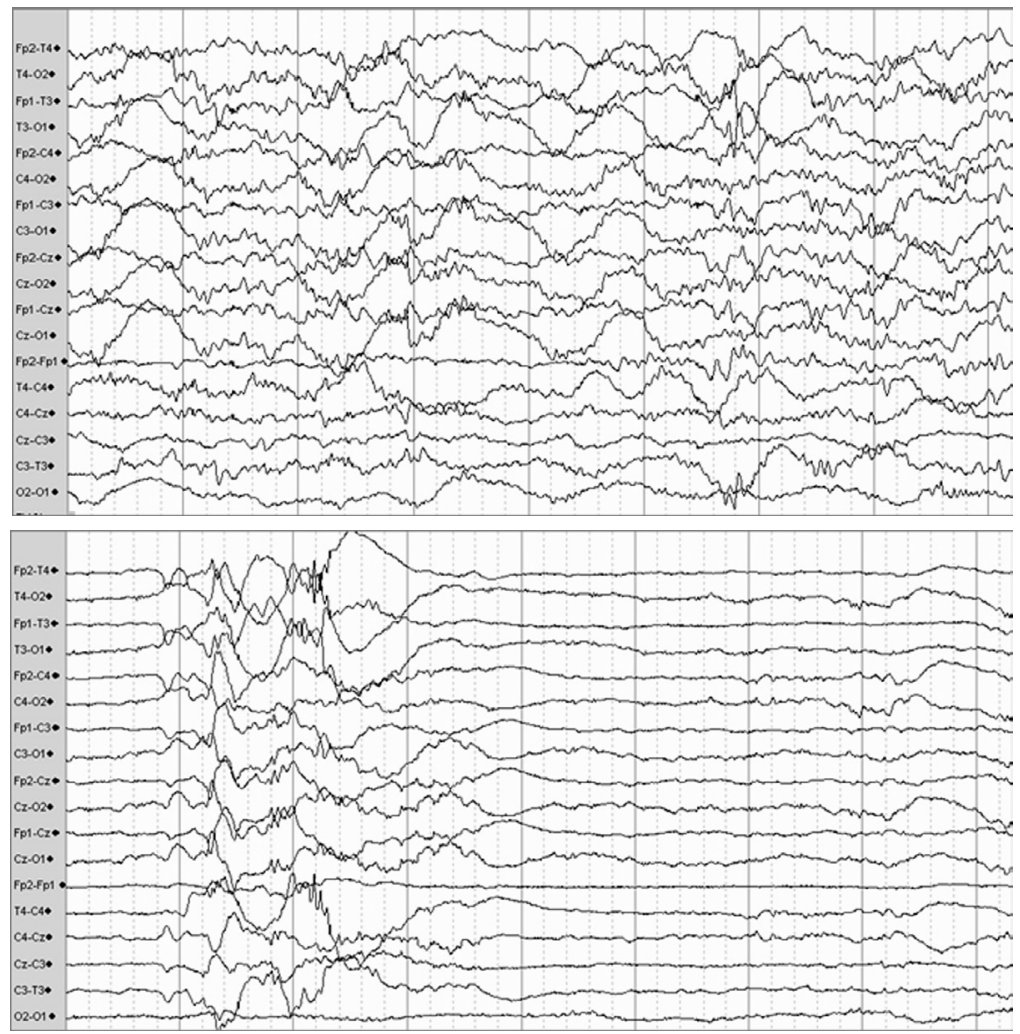

An example of a continuous (A) and a discontinuous (B) pattern in an infant with PMA of 30 weeks. 
Table 1: Continuity and PMA.

\begin{tabular}{|c|c|c|c|c|}
\hline Study & $\begin{array}{l}\text { Definitions } \\
\text { Continuous/Discontinuous } \\
\text { Background Pattern }\end{array}$ & $\begin{array}{l}\text { PMA } \\
(w k)\end{array}$ & $\mathbf{N}$ & $\begin{array}{l}\text { Discontinuity } \\
\text { (\%) }\end{array}$ \\
\hline \multirow[t]{3}{*}{ Anderson 1985 [29] } & \multirow[t]{3}{*}{ Unknown, qualitative } & 27 & 8 & $62 \%$ [11-90] \\
\hline & & 29 & 11 & $53 \%[0-87]$ \\
\hline & & 31 & 14 & $45 \%[0-100]$ \\
\hline \multirow[t]{3}{*}{ * Connel 1987 [68] } & \multirow[t]{3}{*}{ Qualitative scoring system } & 28 & 44 & $28 \%[15-45]$ \\
\hline & & 30 & 44 & $18 \%[5-35]$ \\
\hline & & 32 & 44 & $15 \%[0-28]$ \\
\hline \multirow[t]{3}{*}{ * Van Sweden 1991[69] } & \multirow[t]{3}{*}{ Qualitative scoring system } & 27 & 20 & $53 \%[25-85]$ \\
\hline & & 30 & 20 & $38 \%[5-65]$ \\
\hline & & 32 & 20 & $25 \%[0-53]$ \\
\hline \multirow[t]{4}{*}{ Goto 1992 [70] } & \multirow[t]{4}{*}{ Qualitative scoring system } & 26 & 2 & $67 \%$ \\
\hline & & 28 & 5 & $58 \%$ \\
\hline & & 30 & 6 & $37 \%$ \\
\hline & & 32 & 10 & $20 \%$ \\
\hline \multirow[t]{3}{*}{ Hayakawa 2001 [71] } & Discontinuous pattern: & $21-22$ & 3 & $69 \%[30-96]$ \\
\hline & Burst $(>100 \mu \mathrm{V} ; 2-20 s)$ alternated & $23-24$ & 7 & $54 \%[33-77]$ \\
\hline & with $(\mid \mathrm{BB}<20 \mu \mathrm{V} ;>5 \mathrm{~s})$ & $25-26$ & 5 & $48 \%$ [19-49] \\
\hline
\end{tabular}

Footnote: Studies ordered by year of publication. Postmenstrual age (PMA) expressed in weeks (wk); discontinuity expressed as a mean value in percentage with range between brackets; * data extracted from figures in referring article.

$(<5 \mu \mathrm{V})$ during the quiescent periods, no variation and minimal reaction to stimulation. The BS pattern is always a pathologic pattern and typically observed in full-term asphyxiated infants [18].

To investigate changes within the discontinuous pattern with increasing PMA, burst and IBI lengths were measured. The bursts and IBI's were defined by amplitude and time criteria (tables 2 and 3). Several studies demonstrated that the discontinuous pattern becomes less discontinuous, i.e. mean and maximum burst length increased and/or mean and maximum IBI-length decreased (tables 2 and 3). However, there is great variation in and between these studies. For instance, burst lengths varied between 2 and $90 \mathrm{~s}$ in identical PMA groups and some studies did not show a relation between PMA and burst length at all (table 2).

On the other hand, all authors who studied IBI found a decrease in mean and maximum IBI length with PMA but with considerable variance in and between studies (figure 2; table 3). The variance can be explained by several factors. First, quantitative definitions of burst and IBI varied from study to study. This will obviously lead to differences in results. For instance, Hahn [15], who used the lowest cerebral electrical threshold value $(<15 \mu \mathrm{V})$ for IBI, measured the shortest IBI-lengths.

Additionally, extreme long burst lengths were found in studies in which only an amplitude and no maximum time criterion for the definition of burst was applied. These periods may actually be periods of continuous background activity.

Second, another explanation may be related to the visual interpretation itself. Although burst 
Table 2: Mean, minimum or maximum burst length and PMA.

\begin{tabular}{|c|c|c|c|c|c|}
\hline Study & $\begin{array}{l}\text { Definition } \\
\text { - Amplitude }(\mu \mathrm{V}) \\
\text { - Duration }(\mathrm{s}) \\
\text { - Channels }(\%)\end{array}$ & $\begin{array}{l}\text { PMA } \\
(w k)\end{array}$ & $\mathbf{N}$ & $\begin{array}{l}\text { Burst length (s) } \\
\text { Mean value }\end{array}$ & $\begin{array}{l}\text { Burst length (s) } \\
\text { Minimum \# } \\
\text { Maximum \$ }\end{array}$ \\
\hline \multirow[t]{3}{*}{ Anderson 1985 [29] } & $?$ & 27 & 8 & $5[3-10]$ & \\
\hline & $?$ & 29 & 11 & $4[3-6]$ & \\
\hline & $?$ & 31 & 14 & $5[3-6]$ & \\
\hline \multirow[t]{3}{*}{ Eyre 1988 [72] } & $>50$ & $26-42$ & 25 & $?[2-5]$ & \\
\hline & $2-20$ & & & & \\
\hline & $?$ & & & & \\
\hline \multirow[t]{4}{*}{ Biagioni 1994 [26] } & $>30$ & $27-28$ & 7 & & $1.50 \pm 0.38 \#$ \\
\hline & $?$ & $29-30$ & 15 & & $1.75 \pm 0.60 \#$ \\
\hline & $\geq 50$ & $31-32$ & 28 & & $1.93 \pm 0.64 \#$ \\
\hline & & $33-34$ & 27 & & $2.42 \pm 0.42 \#$ \\
\hline \multirow[t]{3}{*}{ Selton 2000 [17] } & $>30$ & 26 & 4 & [1-131.2] & \\
\hline & $>1$ & 27 & 9 & {$[1-179.0]$} & \\
\hline & $\geq 10$ & 28 & 4 & [1.4-159] & \\
\hline \multirow[t]{3}{*}{ Hayakawa 2001 [71] } & $>100$ & $21-22$ & 3 & $4.3[3.4-5.1]$ & \\
\hline & $2-20$ & 23-24 & 7 & $5.0[3.9-7.4]$ & \\
\hline & $\geq 5$ & $25-26$ & 5 & $5.8[4.9-7.2]$ & \\
\hline \multirow[t]{6}{*}{ Vecchierini 2003 [27] } & $>50$ & $24-27$ & 10 & & $50 \pm 20 \$$ \\
\hline & $>1$ & & & & \\
\hline & $\geq 66$ & & & & \\
\hline & $15-50$ & & & & $24 \pm 6 \$$ \\
\hline & $>1$ & & & & \\
\hline & $\geq 66$ & & & & \\
\hline
\end{tabular}

Footnote: Studies ordered by year of publication. Postmenstrual age (PMA) expressed in weeks (wk). The definition of burst contains amplitude $(\mu \mathrm{V})$, duration (s) and the percentage of channels. The burst length is presented as mean values with ranges between brackets as well as minimum (\#) or maximum (\$) value \pm SD.

and IBI were well defined by quantitative criteria, the actual detection of burst and IBI took place by visual inspection of the EEG tracings by various neurophysiologists. The burst- and IBI- lengths were measured manually. As this is very time consuming and neurophysiologists may not agree if a period of EEG activity is a burst or an artifact with high amplitude (and therefore quantitatively qualifies as burst) or a short period of continuous activity, this may lead to significant variability in results.

Third, as visual analysis of EEG is very time consuming, most studies analyzed only short (15-30 
Table 3: Mean and maximum IBI length and PMA.

\begin{tabular}{|c|c|c|c|c|c|}
\hline Study & $\begin{array}{l}\text { Definition } \\
\text { Amplitude }(\mu \mathrm{V}) \\
\text { Duration }(\mathrm{s})\end{array}$ & $\begin{array}{l}\text { PMA } \\
\text { (wk) }\end{array}$ & $\mathbf{N}$ & $\begin{array}{l}\text { Mean IBI } \\
\text { (s) }\end{array}$ & $\begin{array}{l}\text { Max IB| } \\
\text { (s) }\end{array}$ \\
\hline \multirow[t]{3}{*}{ Anderson 1985 [29] } & $?$ & $27-28$ & 8 & 12 [8-16] & 48 [15-88] \\
\hline & $?$ & $29-30$ & 11 & $9[5-14]$ & 36 [16-57] \\
\hline & & $31-32$ & 14 & 7 [4-11] & $20[6-41]$ \\
\hline \multirow[t]{5}{*}{ Hahn * 1989 [15] } & $<15$ & $26-27$ & 5 & $4[3-4]$ & 9 [7-11] \\
\hline & $>2$ & $28-29$ & 15 & $3[3-4]$ & 8 [7-10] \\
\hline & & $30-31$ & 24 & $3[3-3]$ & $7[6-8]$ \\
\hline & & $32-33$ & 21 & $3[3-3]$ & $4[4-5]$ \\
\hline & & $34-35$ & 20 & $3[3-3]$ & $3[3-4]$ \\
\hline \multirow[t]{4}{*}{ Benda * 1989[28] } & $?$ & $25-26$ & 4 & 22 [11-32] & \\
\hline & $?$ & $27-28$ & 19 & 9 [4-31] & \\
\hline & & $29-30$ & 17 & $8[4-17]$ & \\
\hline & & $31-32$ & 9 & 8 [?-?] & \\
\hline \multirow[t]{4}{*}{ Biagioni 1994 [26] } & $<30$ & $27-28$ & 13 & & $31 \pm 14$ \\
\hline & $?$ & $29-30$ & 15 & & $30 \pm 21$ \\
\hline & & $31-32$ & 28 & & $20 \pm 10$ \\
\hline & & $33-34$ & 29 & & $15 \pm 4$ \\
\hline \multirow[t]{3}{*}{ Victor 2001 [24] } & $<30$ & $26-27$ & 9 & $6[4-13]$ & 15 [11-28] \\
\hline & $?$ & $27-28$ & 10 & $6[4-8]$ & 14 [7-22] \\
\hline & & $29-30$ & 3 & $5[5-8]$ & 12 [11-23] \\
\hline \multirow[t]{3}{*}{ Hayakawa 2001 [71] } & $<30$ & $21-22$ & 3 & $26[14-46]$ & $126[67-218]$ \\
\hline & $>5$ & $23-24$ & 7 & 18 [11-25] & 87 [43-136] \\
\hline & & $25-26$ & 5 & 13 [10-16] & 44 [19-76] \\
\hline \multirow[t]{6}{*}{ Conde 2005 [22] } & $<30$ & $27-28$ & 12 & & $28 \pm 10$ \\
\hline & $?$ & & & & \\
\hline & & $29-30$ & 21 & & $19 \pm 6$ \\
\hline & & $31-32$ & 26 & & $13 \pm 4$ \\
\hline & & $33-34$ & 41 & & $8 \pm 4$ \\
\hline & & $35-36$ & 50 & & $5 \pm 3$ \\
\hline
\end{tabular}

Footnote: Studies ordered by year of publication. Postmenstrual age (PMA) expressed in weeks (wk). The definition of interburst interval (IBI) contains amplitude $(\mu \mathrm{V})$ and duration $(\mathrm{s})$. The IBI length is presented as mean value [range]. The maximum IBI length is expressed as mean [range] or mean \pm SD. 
Figure 2: The correlation between IBI and PMA.

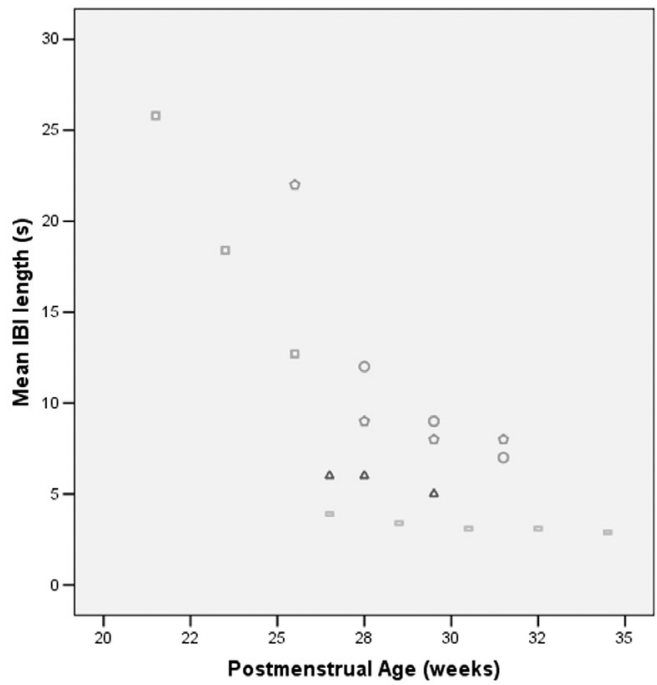

Study

$\square$ Hayalawa $(71)$

Anderson (29)

$\triangle$ Victor (24)

$\triangle$ Benda (28)

口 Hahn (15)

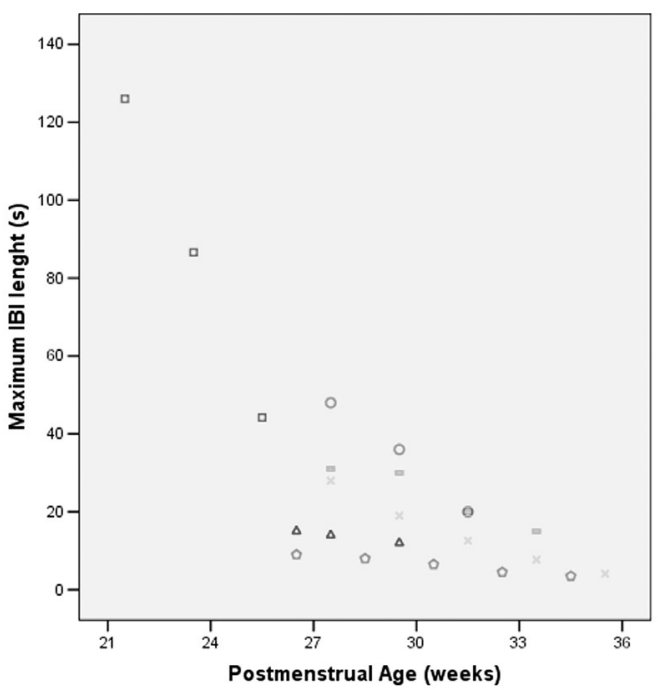

Study

$\square$ Hayakawa $(71)$

Anderson (29)

$\triangle$ Victor (24)

$\triangle \operatorname{Hahn}(15)$

$\square$ Biagioni (26)

Conde (22)

Decrease in mean and maximum IBI with progressing PMA. Data are extracted form several authors and indicated by citation.

min) segments of EEG recordings. New insights show that even in very preterm infants, ultradian cyclic changes in EEG are present[19-21]. The choice of the analyzed EEG epoch within this cycle may greatly influence results. Analyzing longer periods of EEG will minimize the confounding effects of this cyclicity.

Fourth, in the early studies performed in the pre-surfactant era, infants with cerebral pathology were not excluded in maturational studies. Also, these infants often received sedative drugs, which may influence EEG recordings. 
Figure 3: The effect of GA and PMA on IBI.

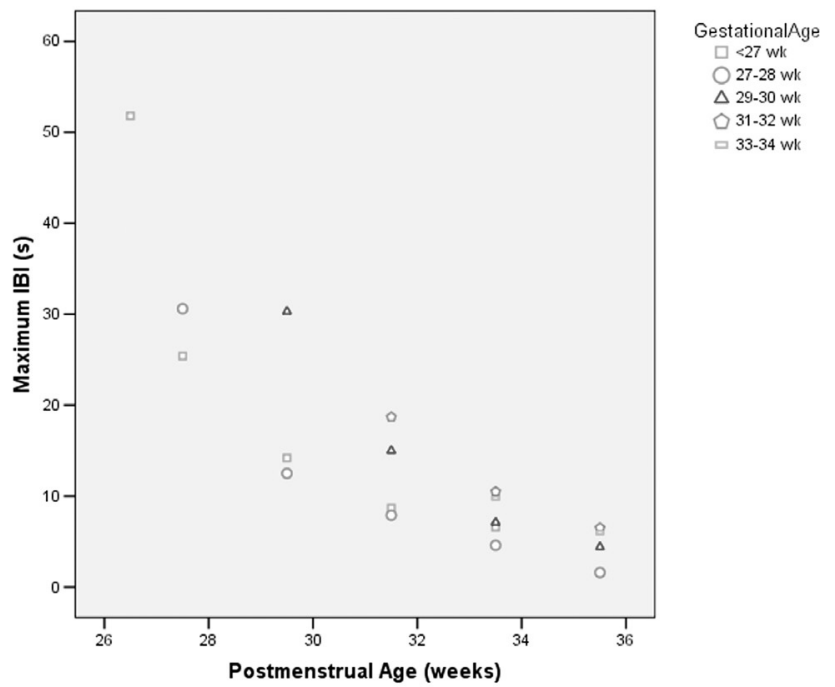

The decrease of maximum IBI lengths of infants born at different gestational age; data extracted from Conde (22) and reconstructed as a figure.

Fifth, a preterm infant at 30 weeks PMA and born at 26 weeks GA may neurophysiologically differ from a preterm infant at 30 weeks PMA and born at 29 weeks GA. Conde et al. [22] demonstrated that maximum IBI lengths were not only influenced by PMA but also by GA, leading to considerable variance between these IBI lengths within similar age groups (figure 3 ).

The exact physiological explanation of the changes in EEG continuity remains to be elucidated. It is suggested that the shortening of IBI and the increase in burst length were related to the maturation in excitatory synapses and/or the loss of presumed sub-cortical systems responsible for the abrupt inhibition of cortical activity $[15,23,24]$. Recently, Biagioni demonstrated that the shortening of IBI intervals is correlated to cortical folding in preterm infants [25]. However, this relation is not necessarily causative.

Minimum burst duration is related to outcome. Different authors found shorter burst durations in preterm infants with major ultrasound lesions and adverse neurological outcome [22, 26]. IBI lengths are related to adverse outcome and neurosonographic abnormalities as well. Scher [10] proposed the $30 / 20$ rule, stating that an interval should not exceed $30 \mathrm{~s}$ on multiple occasions for preterm infants with $\mathrm{PMA}<30$ weeks. As the infant matures beyond 30 weeks PMA the IBI's should be $<20 \mathrm{~s}$.

Vecchierini [27] stated that in neurologically normal (without abnormalities on the cerebral ultrasound and normal neurological examinations) infant at 24-26 weeks PMA, IBI never exceeds $60 \mathrm{~s}$. Benda [28] demonstrated in a prospective study that prolonged inactive phases $(>30 \mathrm{~s})$ at 25-35 weeks PMA were correlated with intra-ventricular hemorrhages (IVH) and mortality. However, all infants in this study received phenobarbital and 13 of the 44 infants did not survive. Hahn $[11,15]$, studying IBI in infants with GA $>26$ weeks and normal outcome at 3 years, found IBI's no longer than $20 \mathrm{~s}$. In infants with $>30$ weeks PMA, the longest measured IBI was only $11 \mathrm{~s}$. Conde [22] observed significantly longer IBI's in preterm infants with major cranial ultrasound lesions. 


\section{Development of specific waveforms}

The EEG signal of the preterm infant consists of waves with different frequency ranges and distribution. Generally, the EEG of the very preterm infant exists of high amplitude slow waves. When the infant matures, a development towards lower amplitude, faster waves is observed [17]. Different wave patterns, which are characteristic for a certain period within the PMA, have been described:

- Delta wave: Slow waves with frequency of o-4 $\mathrm{Hz}$ and amplitude of 50-250 $\mu \mathrm{V}$. Below 28 weeks PMA, the slow waves have the highest amplitude in the occipital areas and are isolated to one or two wave forms and are rarely longer than 5 to $6 \mathrm{~s}$. Rhythmic delta waves are seen particularly in the central midline locations. With increasing PMA, the occipital waves become quite prominent, with durations exceeding $1 \mathrm{~min}$, also referred to as occipital predominance. The occipital predominance disappears after 34 weeks PMA [10, 29, 30]. Biagioni noted a decreasing trend in amplitude of delta waves from 27 to 34 weeks [26].

- Delta brushes: Superimposed rapid alpha $(8-12 \mathrm{~Hz})$ and beta $(13-30 \mathrm{~Hz})$ activity on delta waves, usually on the ascending slope. The amplitude of the activity rarely exceeds $60-75 \mu \mathrm{V}$. Delta brushes are first observed around 26 weeks PMA in the vertex and central region, increase in number until 32 weeks PMA, when they are observed in the temporal and occipital region. They diminish between 34 and 37 weeks. If brushes are still present in the term infant, they are always occipital $[10,31,32]$. Brushes are more frequent during active sleep until 32 weeks. However, after 32 weeks they are more frequent during quiet sleep [30].

- Sharp theta rhythms on the occipital areas of the premature (so-called STOP): short rhythmic burst of sharp theta $(4-7 \mathrm{~Hz})$ waves in the occipital region. These are typically present between 24 and 26 weeks PMA and are mostly unilateral, little higher in frequency and lower in amplitude than temporal saw tooth [33]. Also named monorhythmic alpha/theta rhythm by Scher [10, 31]. A similar pattern, named occipital saw tooth, was described [34].

- Temporal sawtooth: rhythmic bursts of sharp theta $(4-7 \mathrm{~Hz})$ waves in the temporal region with amplitude of 100-250 $\mu \mathrm{V}$. These waves typically appear at 26 weeks, attain their maximum at 29-30 weeks PMA and disappear at 32 weeks PMA. Presence of temporal saw tooth at 33-34 weeks PMA is correlated to adverse outcome $[17,26,35]$. This activity must be distinguished from the repetitive sharp activity in the theta range seen at near-term and term ages in the midline and Rolandic areas $[10,30]$. The temporal waves in the occipital region (26-28 weeks PMA) precede the temporal wave in the temporal region and seem to mature earlier (decrease in amplitude and increase in frequency) $[24,27,34]$.

- Anterior slow dysrhythmia: short burst of mono- or polyphasic delta waves at 1-3 $\mathrm{Hz}$ with voltage of 50-100 $\mu \mathrm{V}$ over the frontal region. Appear at 35-37 weeks during active sleep [30,32].

In order to quantify frequency aspects of the preterm EEG, spectral analysis has been used (table 4). Spectral analysis of a signal involves decomposition of the signal into (co)sinusoidal components [36]. Almost all authors who studied frequency aspects of the EEG of the preterm infant used the Fast Fourier Transform (FFT). As Fourier transform requires a stationary signal, adaptive segmentation before spectral analysis is required to create "quasi-stationary" EEG epochs. 
Table 4: Spectral power analysis and PMA.

\begin{tabular}{|c|c|c|c|c|c|}
\hline Study & $\begin{array}{l}\text { PMA } \\
(w k)\end{array}$ & $\mathbf{N}$ & $\begin{array}{l}\text { Band definition } \\
\text { Absolute or relative } \\
\mathrm{Hz}\end{array}$ & $\begin{array}{l}\text { Result } \\
\downarrow \text { or } \uparrow \text { with } \\
\text { PMA }\end{array}$ & $\begin{array}{l}\text { Correlation coef- } \\
\text { ficient or Kruskal- } \\
\text { Wallis }(\$)\end{array}$ \\
\hline \multirow[t]{8}{*}{ Bell [73] } & $26-41$ & 60 & Absolute $\delta 1:<1$ & $\downarrow$ & -0.75 \\
\hline & & & Absolute $\delta 2: 1-4$ & $\downarrow$ & -0.35 \\
\hline & & & Absolute $\beta$ : 14-30 & $\downarrow$ & -0.11 \\
\hline & & & Relative $\delta 1:<1$ & $\downarrow$ & -0.72 \\
\hline & & & Relative $\delta 2: 1-4$ & $\uparrow$ & +0.58 \\
\hline & & & Relative $\theta$ band: 4-7 & $\uparrow$ & +0.76 \\
\hline & & & Relative a band: 8-13 & $\uparrow$ & +0.68 \\
\hline & & & Relative $\beta$ band: $14-30$ & $\uparrow$ & +0.46 \\
\hline \multirow[t]{3}{*}{ Okumura $[40,43]$} & $29-34$ & 30 & Absolute $\delta 1:<1$ & $\downarrow$ & $p<0.05(\$)$ \\
\hline & & & Absolute $\delta 2-4: 1-4$ & $\downarrow$ & $p<0.05(\$)$ \\
\hline & & & Absolute $\theta: 4-7$ & $\downarrow$ & $p<0.05(\$)$ \\
\hline \multirow[t]{6}{*}{ Scher [38] } & $28-36$ & 52 & Total power: $0.5-30$ & $\downarrow$ & -0.71 \\
\hline & & & Absolute $\delta: 0.5-4$ & $\downarrow$ & -0.63 \\
\hline & & & Absolute $a$ and $\beta: 8-30$ & $\uparrow$ & +0.43 \\
\hline & $36-44$ & 54 & Absolute $\theta: 4-7$ & $\uparrow$ & +0.31 \\
\hline & & & Absolute a: 8-13 & $\downarrow$ & -0.74 \\
\hline & & & Absolute $\beta: 14-30$ & $\downarrow$ & $-0,77$ \\
\hline
\end{tabular}

The exact technique (e.g. overlapping, detrending and tapering) varied between studies or the method was not described in detail. For instance, the epochs subjected to transform varied from 2 to $20 \mathrm{~s}$. The sample frequency varied between $64-80 \mathrm{~Hz}$ in earlier studies by Scher [37] to $256 \mathrm{~Hz}$ in a more recent study by Victor [24].

After FFT, the spectral power of a certain frequency band is expressed in absolute (in $\mu \mathrm{V}_{2}$ ) and/ or relative power (as a percentage of the total power of all frequency bands).

Scher demonstrated in bi-weekly sequential EEG recordings in 56 preterm infants with GA < 32 weeks that total spectral power and delta $(1-4 \mathrm{~Hz})$ power decreased with PMA [38]. Bell and Okumura subdivided the delta frequency band in 2 and 4 bands, respectively [39, 40]. Both found an absolute reduction of power in the delta1 band $(<1 \mathrm{~Hz})$. However, Okumura demonstrated a reduction in power of the faster $(>1-4 \mathrm{~Hz})$ delta frequencies, which took only place in the occipital areas, suggesting regional differences in EEG maturation (table 4). 
Other authors also found occipital to temporal maturational difference when analyzing temporal waves, i.e. more mature temporal waves in occipital regions compared to the temporal regions [17]. (However, these waves were detected by visual analysis only). This gradient in occipito-temporal neurophysiological maturation is in agreement with findings in recent MRI studies of preterm infants. These studies demonstrate that cortical maturation and gyration of the occipital regions precedes that of the frontal and temporal regions [41, 42].

Okumura studied faster frequencies in infants in the age range of 29-34 weeks PMA as well, observing a decrease in absolute theta power with progressing PMA, but no significant changes in the alpha $(8-12 \mathrm{~Hz})$ and beta $(13-30 \mathrm{~Hz})$ bands [43]. Scher however, found a positive relation between the absolute alpha and beta power and PMA [38]. Bell showed a quadratic relationship between absolute beta power and PMA with a maximum at 32-34 weeks [39].

In addition, the spectral edge frequency can be calculated with FFT. This is the frequency that delimits 90 or $95 \%$ of the total spectral power $[24,44]$. Although there is a significant decrease in spectral edge frequency during the first days of life in preterm infants, no relation between spectral edge frequency and PMA has been demonstrated.

Fourier transform, the most commonly used method to analyze variability, does not offer an easy assessment of dynamics because of limitations inherent in its stationary hypothesis. In contrast, wavelet transform allows analysis of non stationary signals and offers two complementary interesting features. First, wavelet transform allows a temporally localized analysis of the signal, thus giving access at any time to the status of the EEG signal, as, for example, when the balance of the nervous system equilibrium is suddenly modified by sleep state change. Second, the shape of the wavelet transform-analyzing equation (so-called mother wavelet) differs from the fixed sinusoidal shape of the Fourier transform and can be designed to better fit the shape of the analyzed signal, allowing a better quantitative measurement. Therefore, wavelet transform is suggested to be a more suitable technique than Fourier transform [36]. Wavelet transform was successfully used by Turnbull to detect tracé alternant, one of the five sleep stages in term infants [45]. To our knowledge, wavelet transform has not been used to analyze the EEG of the preterm infant.

\section{Inter-hemispheric synchrony}

Inter-hemispheric synchrony is defined as simultaneous EEG activity in homologous regions of each hemisphere. If bursts appear within 1.5 or $2 \mathrm{~s}$ from each other in the two hemispheres they are considered synchronous [10,17,18]. Lombroso [16] manually calculated the degree of synchrony in 5 min discontinuous EEG epochs and found a relation between synchrony and PMA (table 5). Until 30 weeks PMA the EEG is hypersynchronous, which may be related to thalamic driving as described by Steriade [46]. From 30-36 weeks PMA the EEG is more asynchronous, which can be explained by cortical synaptogenesis and myelinisation. From 36 weeks onwards an increase in synchrony is observed again, due to an increase in inter-cortical connections between the two hemispheres [10,27].

Aso [47] reported mildly to moderately severe neuropathological lesions in 9 patients with excessively inter-hemispheric asynchrony as compared to normal infants of equal PMA. Similarly, 
Table 5: Inter-hemispheric synchrony and PMA

\begin{tabular}{|c|c|c|}
\hline Author & PMA & Percentage synchrony \\
\hline \multirow[t]{3}{*}{ Anderson et al. [29] } & $27-28$ & $92 \%$ [80-100\%] \\
\hline & $29-30$ & $94 \%$ [79-100\%] \\
\hline & $31-32$ & $94 \%$ [86-100\%] \\
\hline \multirow[t]{7}{*}{ Lombroso et al. [16] } & $26-28$ & $90-100 \%$ \\
\hline & $29-30$ & $80-100 \%$ \\
\hline & $31-32$ & $50-90 \%$ \\
\hline & $33-34$ & $60-80 \%$ \\
\hline & $35-36$ & $70-85 \%$ \\
\hline & $37-39$ & $80-100 \%$ \\
\hline & $40-42$ & $100 \%$ \\
\hline
\end{tabular}

Footnote: Postmenstrual age (PMA) expressed in weeks (wk). The last column shows the percentage of synchrony, i.e. the amount of burst appearing within $<2 \mathrm{~s}$ in both hemispheres.

Table 6: Sleep-wake patterns in full-term newborns.

\begin{tabular}{|c|c|c|c|c|}
\hline Pattern & $\begin{array}{l}\text { Amplitude } \\
(\mu \mathrm{V})\end{array}$ & Continuity & $\begin{array}{l}\text { Frequencies } \\
(\mathrm{Hz})\end{array}$ & Sleep state \\
\hline \multirow[t]{2}{*}{ Low voltage irregular } & $20-30$ & continuous & $5-8$ & Active \\
\hline & & & $1-5$ & \\
\hline \multirow[t]{3}{*}{ Trace alternant } & Burst \& |B| & discontinuous & Burst: 0.5-3 & Quiet \\
\hline & No exact amplitude & & IBI: not defined & \\
\hline & criteria & & & \\
\hline High Voltage Slow & $50-150$ & continuous & $0.5-4$ & Quiet \\
\hline \multirow[t]{2}{*}{ Mixed } & High \& low voltage & continuous & $1-10$ & Active \\
\hline & components & & & \\
\hline
\end{tabular}

Footnote: Sleep-wake patterns in full-term newborns as described by Stockard-Pope [74].

asynchronous recordings have been described in congenital hydrocephalus [48]. Tharp [49] reported 6 premature infants with marked asynchrony, of which 5 died or suffered severe neurological sequelae. Inter-hemispheric asynchrony has also been suggested to be a useful parameter to assess EEG dysmaturity $[11,18]$.

In addition, spectral correlation can be calculated $\left(\mathrm{C}_{3}-\mathrm{T}_{3} / \mathrm{C}_{4}-\mathrm{T}_{4}\right)$ as measure of symmetry between the two hemispheres. If the degree of correlation between 2 EEG signals (on opposite sites of the brain) is high, the electrical potentials associated with these 2 channels are synchronized and have similar shapes [50]. From 36 weeks PMA towards term there is an increase in spectral correlation. 
It is suggested that the increase in spectral correlations represents the formation of intercortical connections at the corpus callosum [51].

\section{Development of sleep-wake cycling}

An important aspect of neurophysiologic maturation of the preterm infant is the development of sleep-wake behavior. Sleep states can be defined by a combination of typical EEG patterns in combination with physiologic and autonomic behaviors.

At 28 to 30 weeks PMA, the background patterns become associated with the emerging rudimentary sleep states $[19,20]$. The continuous EEG pattern becomes related to active (REM) sleep and the discontinuous pattern becomes linked to quiet (NREM) sleep. However, the other physiologic behaviors that are related to REM and NREM sleep in full-term infants are not linked yet, leaving the sleep states difficult to determine at this age. The intermediate or transitional sleep is used to indicate periods of sleep in which lack of concordance between physiologic sleep criteria does not allow their classification as either active or quiet [31].

In a study by Scher to investigating sleep cyclicity in infants with PMA < 30 weeks, a quantitative definition for quiet sleep state was suggested, based on two criteria: 1. the percentage of EEG discontinuity and 2. the number of rapid eye movements (REM's). However both criteria were detected only visually [31].

As the infant matures from 30 to 36 weeks, the different physiologic sleep behaviors become more coalescent with the active and quiet sleep states. In another study by Scher in infants at PMA 29-36 weeks, the degree of EEG discontinuity (also detected visually) was related to 1. decreased REM's, 2. decreased numbers of spontaneous arousals, 3. increased spectral theta energies and 4 . decreased facial movements, independent of PMA [52].

At 36 weeks PMA, a well-defined sleep cycle with a duration of 30-70 min can be observed. During this cycle, four different background patterns can be distinguished of which three are continuous and only one is discontinuous (table 6). The latter is now named tracé alternant [20, 53]. During the different sleep states, the EEG background shows high correlation with other physiologic sleep behaviors, like respiratory rate, heart rate and REM's [51]. Several efforts were made to detect different sleep states automatically in the term infant. For example, Turnbull developed an algorithm to detect tracé alternant automatically in full-term infants with use of wavelet transform [45].

\section{EEG maturation and outcome}

Lombroso [16] stated that when features from the EEG sleep pattern run behind the actual PMA, the EEG is called "dysmature". Following this, Tharp [12] studied the EEGs of 36 preterm infants with bronchopulmonary dysplasia. He considered the EEGs to be dysmature if they, by visual analysis appeared 2-3 weeks younger than the actual PMA. He observed that EEG dysmaturity was related to adverse outcome later in life (measured with Bailey and Stanford score at 1,2 and 3 years of age). However, Tharp only detected dysmaturity in EEG recordings performed at PMA > 37 weeks. 
He suggested that dysmaturity could not be detected at lower PMA's because of wider ranges in normal values. Most importantly, the exact criteria he used to detect dysmaturity were not mentioned.

Studies in the last decade have assessed dysmaturity at lower PMA. In different studies a Japanese study group defined two types of chronic EEG abnormalities in preterm infants: the disorganized and dysmature pattern [54-57].

The disorganized pattern refers to an abnormal, deformed background activity without definite findings of acute depression. This is most typically represented in deformed "mechanical" cogwheel shaped delta brushes and sharp transients. Mostly, the disorganized pattern follows after a period of severe EEG background depression, which is caused by a brain insult in the perinatal period [55].

The dysmature pattern is defined as an EEG with some patterns immature for PMA. Most important findings suggesting a dysmature EEG pattern are:

- persistence of high voltage temporal theta bursts after 33 weeks PMA.

- persistence of extremely slow delta activities after 33 weeks PMA.

- persistence of delta brushes after 39 weeks PMA.

- persistence of inter-hemispheric asynchrony in tracé alternant.

- persistence of discontinuous tracing after 39 weeks PMA.

- poor development of mature high voltage slow pattern.

Okumura [56] suggested that the disorganized pattern was related to white matter injury, like intraventricular hemorrhage and periventricular leucomalacia and was related to adverse outcome in motor skills. The dysmature pattern is thought to be related to grey matter injury and an adverse cognitive outcome. Indeed, the relation between the volume of cortical gray matter of preterm infants at term and cognitive outcome later in life was demonstrated in different volumetric 3D Magnetic Resonance Imaging studies [58-60]. Biagioni [61] studied EEG dysmaturity in preterm infants (28-34 weeks PMA) using a semi-quantitative scoring system, in which temporal sawtooth, burst lengths and delta brushes were visually counted. He also demonstrated the relationship between EEG dysmaturity and neurological outcome at one year follow-up. Interestingly, there was a low correlation between EEG dysmaturity and ultrasound abnormalities.

Scher has studied EEG dysmaturity extensively in preterm infants [28, 53, 62-64]. He developed a model, in which not only EEG aspects were included but also other physiological sleep behaviors which correlate with increasing PMA. Most fascinatingly, some of these behaviors are delayed (i.e. the development appears to be two weeks younger than actual PMA) and some are accelerated (development appears to be two weeks older than the actual PMA). This reflects a disorganized brain development. Using a complex analytic procedure, Scher was able to calculate a 'dysmaturity index' for preterm infants at term, which also correlated with outcome at 24 months. However, this model could only be implemented at term age and not at earlier stages, when the developing brain is most vulnerable to environmental adverse factors. Also, this method is very complex and therefore not easy to apply in the neonatal intensive care unit. 
Table 7: Proposal of quantitative analysis of preterm EEG characteristics.

\begin{tabular}{|c|c|c|c|c|}
\hline Neurophysiological definition & $\begin{array}{l}\text { Amplitude } \\
(\mu \mathrm{V})\end{array}$ & $\begin{array}{l}\text { Frequency } \\
(\mathrm{Hz})\end{array}$ & $\begin{array}{l}\text { Duration } \\
\text { (s) }\end{array}$ & Channels \\
\hline Continuous & $>50$ & variable & $>20$ & variable \\
\hline $\begin{array}{l}\text { Discontinuous, } \\
\text { Burst criterion }\end{array}$ & $>50$ & variable & $1-20$ & $\geq 1$ \\
\hline $\begin{array}{l}\text { Discontinuous, } \\
\text { IBI criterion }\end{array}$ & $<30$ & & $\geq 1$ & All \\
\hline$a$ & variable & $8-12$ & variable & variable \\
\hline$\beta$ & variable & $13-30$ & variable & variable \\
\hline$\theta$ & variable & $4-7$ & variable & variable \\
\hline$\delta 1$ & variable & $0.5-1$ & variable & variable \\
\hline$\delta 2$ & variable & $1-4$ & variable & variable \\
\hline
\end{tabular}

\section{The value of quantitative analysis of EEG maturation}

Nowadays all digital EEG recorders provide hardware and software opportunities to analyze longer segments of EEG recordings more objectively. As discussed earlier, these opportunities have been widely explored in other fields of research. However, in the neonatology catching up has to be done. An automatic analysis algorithm may help to set uniform reference values of maturational characteristics of the preterm EEG. It may also be used to monitor neurophysiologic maturation in individual infants longitudinally. Furthermore, the information concerning brain development may become available to the EEG non-expert neonatologist.

Recently, we developed an automated algorithm which detects convulsions in the amplitudeintegrated EEG of full-term asphyxiated infants [65]. In addition, we are evaluating several algorithms with the aim to detect discontinuous periods in the preterm EEG [66] and describe amplitude integrated EEG maturation quantitatively [67]. Therefore, we reviewed different maturational aspects of the preterm EEG and the relation between EEG maturation and outcome later in life. Continuity, frequency, inter-hemispheric synchrony and correlation, and sleep-wake behavior are parameters which can be quantified with an automated analyzing algorithm. As burst and IBI are important aspects of the preterm EEG, change with increasing PMA and are related to outcome, automatic detection of these aspects will be of importance. Burst and IBI can be detected with amplitude, time, and channels criteria as proposed in table 7. After detection, burst can be studied more extensively by automated frequency analysis. Spectral analysis can be performed on specific parts of the EEG (like burst, IBI or continuous tracings), but also on longer recordings. As Fourier transform requires a stationary signal, adaptive segmentation before spectral analysis is required to create "quasistationary" EEG epochs. As discussed earlier, wavelet transform is potential very useful to quantify instantaneous changes of the EEG signal. Definitions of the different frequency 
bands are already universal and described in table 7. As there are regional differences in brain maturation and the connections between hemispheres increase with maturation, it is interesting to study the regional differences or similarities in the preterm EEG. At first, inter-hemispheric synchrony and correlation can be investigated automatically with automatic burst detection and spectral analysis, respectively. But also differences between the occipital and temporal regions (like sawtooth) can be studied by an automated algorithm. At last, the relation between the EEG characteristics and different sleep states and physiologic behaviors such as heart and respiratory rate can be investigated.

However in developing an automated algorithm for preterm EEG's, one important methodological issue has to be addressed. As (especially uncomplicated) preterm infants cannot be instructed and are often in motion and as the EEG's are recorded in an electrical noisy NICU environment, recorded EEGs contain many artifacts. These artifacts have to be detected and/or removed when analyzing the EEG. A reliable algorithm has to be equipped with artifact detection or removal possibilities.

In addition, future research is necessary to develop and validate a reliable algorithm and to correlate EEG maturation with short and long-term outcome. 
1. Cooke RW. Preterm mortality and morbidity over 25 years. Arch Dis Child Fetal Neonatal Ed 2006;91(4):F293-4.

2. Horbar JD, Badger GJ, Carpenter JH, Fanaroff AA, Kilpatrick S, LaCorte $M$, et al. Trends in mortality and morbidity for very low birth weight infants, 1991-1999. Pediatrics 2002;110(1 Pt 1):143-51.

3. Anderson P, Doyle LW. Neurobehavioral outcomes of school-age children born extremely low birth weight or very preterm in the 1990 s. Jama 2003;289(24):3264-72.

4. Bhutta AT, Cleves MA, Casey PH, Cradock MM, Anand KJ. Cognitive and behavioral outcomes of school-aged children who were born preterm: a meta-analysis. Jama 2002;288(6):728-37.

5. Hack M, Fanaroff AA. Outcomes of children of extremely low birthweight and gestational age in the 1990's. Early Hum Dev 1999;53(3):193-218.

6. Perlman JM. Cognitive and behavioral deficits in premature graduates of intensive care. Clin Perinatol 2002;29(4):779-97.

7. Volpe JJ. Neurologic outcome of prematurity. Arch Neurol 1998;55(3):297-300.

8. Gressens $P$, Rogido $M$, Paindaveine $B$, Sola A. The impact of neonatal intensive care practices on the developing brain. J Pediatr 2002;140(6):646-53.

9. Perlman JM. Neurobehavioral deficits in premature graduates of intensive care--potential medical and neonatal environmental risk factors. Pediatrics 2001;108(6):1339-48.

10. Scher MS. Normal electrographic-polysomnographic patterns in preterm and fullterm infants. Semin Pediatr Neurol 1996;3(1):2-12.

11. Tharp BR, Scher MS, Clancy RR. Serial EEGs in normal and abnormal infants with birth weights less than 1200 grams--a prospective study with long term follow-up. Neuropediatrics 1989;20(2):64-72.
12. Tharp BR. Electrophysiological brain maturation in premature infants: an historical perspective. J Clin Neurophysiol 1990;7(3):302-14.

13. Ramesh VJ, Umamaheswara Rao GS. Quantification of burst suppression and bispectral index with 2 different bolus doses of thiopentone sodium. J Neurosurg Anesthesiol 2007;19(3):179-82.

14. Sarkela M, Mustola S, Seppanen T, Koskinen M, Lepola P, Suominen K, et al. Automatic analysis and monitoring of burst suppression in anesthesia. J Clin Monit Comput 2002;17(2):125-34.

15. Hahn JS, Monyer H, Tharp BR. Interburst interval measurements in the EEGs of premature infants with normal neurological outcome. Electroencephalogr Clin Neurophysiol 1989;73(5):410-8.

16. Lombroso CT. Neonatal polygraphy in full-term and premature infants: a review of normal and abnormal findings. J Clin Neurophysiol 1985;2(2):105-55.

17. Selton D, Andre M, Hascoet JM. Normal EEG in very premature infants: reference criteria. Clin Neurophysiol 2000;111(12):2116-24.

18. Holmes GL, Lombroso CT. Prognostic value of background patterns in the neonatal EEG. J Clin Neurophysiol 1993;10(3):323-52.

19. Curzi-Dascalova L, Figueroa JM, Eiselt $M$, Christova E, Virassamy $A$, d'Allest $A M$, et al. Sleep state organization in premature infants of less than 35 weeks' gestational age. Pediatr Res 1993;34(5):624-8.

20. Scher MS. Understanding sleep ontogeny to assess brain dysfunction in neonates and infants. J Child Neurol 1998;13(10):467-74.

21. Scher MS, Johnson MW, Holditch-Davis D. Cyclicity of neonatal sleep behaviors at 25 to 30 weeks' postconceptional age. Pediatr Res 2005;57(6):879-82.

22. Conde JR, de Hoyos AL, Martinez ED, Campo CG, Perez AM, Borges AA. Extrauterine life 
duration and ontogenic EEG parameters in preterm newborns with and without major ultrasound brain lesions. Clin Neurophysiol 2005;116(12):2796-809.

23. Vecchierini-Blineau MF, Nogues B, Louvet $S$, Desfontaines O. Positive temporal sharp waves in electroencephalograms of the premature newborn. Neurophysiol Clin 1996;26(6):350-62.

24. Victor S, Appleton RE, Beirne M, Marson AG, Weindling AM. Spectral analysis of electroencephalography in premature newborn infants: normal ranges. Pediatr Res 2005;57(3):336-41.

25. Biagioni E, Frisone MF, Laroche S, Kapetanakis $B A$, Ricci D, Adeyi-Obe M, et al. Maturation of cerebral electrical activity and development of cortical folding in young very preterm infants. Clin Neurophysiol 2007;118(1):53-9.

26. Biagioni E, Bartalena L, Boldrini A, Cioni G, Giancola S, Ipata AE. Background EEG activity in preterm infants: correlation of outcome with selected maturational features. Electroencephalogr Clin Neurophysiol 1994;91(3):154-62.

27. Vecchierini MF, d'Allest AM, Verpillat P. EEG patterns in 10 extreme premature neonates with normal neurological outcome: qualitative and quantitative data. Brain Dev 2003;25(5):330-7.

28. Benda Gl, Engel RC, Zhang YP. Prolonged inactive phases during the discontinuous pattern of prematurity in the electroencephalogram of very-low-birthweight infants. Electroencephalogr Clin Neurophysiol 1989;72(3):189-97.

29. Anderson CM, Torres F, Faoro A. The EEG of the early premature. Electroencephalogr Clin Neurophysiol 1985;60(2):95-105.

30. Torres F, Anderson C. The normal EEG of the human newborn. J Clin Neurophysiol 1985;2(2):89-103.

31. Holmes GL, Moshe SL, Jones HR. Clinical Neurophysiology of Infancy, Childhood and Adolescense. 1st Edition.: Elsevier; 2005.

32. Pressler RM, Bady B, Binnie CD, Boylan GB, Connell JA. Neurophysiology of the Neonatal period. In: Binnie CD, editor. Clinical Neurophysiology (Volume 2): Elsevier Science; 2006. p. 450-506.

33. Hughes JR, Miller JK, Fino JJ, Hughes CA. The sharp theta rhythm on the occipital areas of prematures (STOP): a newly described waveform. Clin Electroencephalogr 1990;21(2):77-87.

34. Biagioni E, Frisone MF, Laroche S, Rutherford M, Counsell S, Cioni G, et al. Occipital sawtooth: a physiological EEG pattern in very premature infants. Clin Neurophysiol 2000;111(12):2145-9.

35. Castro Conde JR, Martinez ED, Campo CG, Perez AM, McLean ML. Positive temporal sharp waves in preterm infants with and without brain ultrasound lesions. Clin Neurophysiol 2004;115(11):2479-88.

36. Akin M. Comparison of wavelet transform and FFT methods in the analysis of EEG signals. $J$ Med Syst 2002;26(3):241-7.

37. Scher MS, Steppe DA, Banks DL. Prediction of lower developmental performances of healthy neonates by neonatal EEG-sleep measures. Pediatr Neurol 1996;14(2):137-44.

38. Scher MS, Steppe DA, Banks DL, Guthrie RD, Sclabassi RJ. Maturational trends of EEG-sleep measures in the healthy preterm neonate. Pediatr Neurol 1995;12(4):314-22.

39. Bell AH, McClure BG, McCullagh PJ, McClelland $R J$. Variation in power spectral analysis of the EEG with gestational age. J Clin Neurophysiol 1991;8(3):312-9.

40. Okumura A, Kubota T, Toyota N, Kidokoro H, Maruyama K, Kato T, et al. Amplitude spectral analysis of maturational changes of delta waves in preterm infants. Brain Dev 2003;25(6):406-10.

41. Battin MR, MaaloufEF, Counsell SJ, Herlihy AH, Rutherford MA, Azzopardi D, et al. Magnetic resonance imaging of the brain in very preterm infants: visualization of the germinal matrix, early myelination, and cortical folding. Pediatrics 1998;101(6):957-62. 
42. Mewes $A U$, Huppi PS, Als H, Rybicki FJ, Inder TE, McAnulty GB, et al. Regional brain development in serial magnetic resonance imaging of low-risk preterm infants. Pediatrics 2006;118(1):23-33.

43. Okumura A, Kubota T, Tsuji T, Kato T, Hayakawa F, Watanabe K. Amplitude spectral analysis of theta/alpha/beta waves in preterm infants. Pediatr Neurol 2006;34(1):30-4.

44. West $C R$, Harding JE, Williams CE, Gunning MI, Battin MR. Quantitative electroencephalographic patterns in normal preterm infants over the first week after birth. Early Hum Dev 2006;82(1):43-51.

45. Turnbull JP, Loparo KA, Johnson MW, ScherMS. Automated detection of trace alternant during sleep in healthy full-term neonates using discrete wavelet transform. Clin Neurophysiol 2001;112(10):1893-900.

46. Steriade M, Gloor P, Llinas RR, Lopes de Silva FH, Mesulam MM. Report of IFCN Committee on Basic Mechanisms. Basic mechanisms of cerebral rhythmic activities. Electroencephalogr Clin Neurophysiol 1990;76(6):481-508.

47. Aso K, Scher MS, Barmada MA. Neonatal electroencephalography and neuropathology. J Clin Neurophysiol 1989;6(2):103-23.

48. Watanabe K, Yamada H, Hara K, Miyazaki S, NakamuraS. Neurophysiological evaluation of newborns with congenital hydrocephalus. Clin Electroencephalogr 1984;15(1):22-31.

49. Tharp BR, Cukier F, Monod N. The prognostic value of the electroencephalogram in premature infants. Electroencephalogr Clin Neurophysiol 1981;51(3):219-36.

50. Scher MS, Sun M, Steppe DA, Guthrie RD, Sclabassi RJ. Comparisons of EEG spectral and correlation measures between healthy term and preterm infants. Pediatr Neurol 1994;10(2):104-8.

51. Scher MS, Dokianakis SG, Sun M, Steppe $D A$, Guthrie RD, Sclabassi RJ. Computer classification of sleep in preterm and full-term neonates at similar postconceptional term ages. Sleep 1996;19(1):18-25.

52. Scher MS, Dokianakis SG, Steppe DA, Banks $D L$, Sclabassi RJ. Computer classification of state in healthy preterm neonates. Sleep 1997;20(2):132-41.

53. Scher MS, Turnbull J, Loparo K, Johnson MW. Automated state analyses: proposed applications to neonatal neurointensive care. J Clin Neurophysiol 2005;22(4):256-70.

54. Hayakawa F, Okumura A, Kato T, Kuno K, Watanabe K. Disorganized patterns: chronic-stage EEG abnormality of the late neonatal period following severely depressed EEG activities in early preterm infants. Neuropediatrics 1997;28(5):272-5.

55. Hayakawa F, Okumura A, Kato T, Kuno K, Watanabe K. Dysmature EEG pattern in EEGs of preterm infants with cognitive impairment: maturation arrest caused by prolonged mild CNS depression. Brain Dev 1997;19(2):122-5.

56. Okumura A, Hayakawa F, Kato T, Kuno K, Watanabe K. Developmental outcome and types of chronic-stage EEG abnormalities in preterm infants. Dev Med Child Neurol 2002;44(11):729-34.

57. Watanabe K, Hayakawa F, Okumura A. Neonatal EEG: a powerful tool in the assessment of brain damage in preterm infants. Brain Dev 1999;21(6):361-72.

58. Inder TE, Warfield SK, Wang H, Huppi PS, Volpe JJ. Abnormal cerebral structure is present at term in premature infants. Pediatrics 2005; 115(2):286-94.

59. Peterson BS, Anderson AW, Ehrenkranz R, Staib $L H$, Tageldin M, Colson E, et al. Regional brain volumes and their later neurodevelopmental correlates in term and preterm infants. Pediatrics 2003;111(5 Pt 1):939-48.

60. Peterson BS, Vohr B, Staib LH, Cannistraci CJ, Dolberg A, Schneider KC, et al. Regional 
brain volume abnormalities and long-term cognitive outcome in preterm infants. Jama 2000;284(15):1939-47.

61. Biagioni E, Bartalena L, Biver P, Pieri R, Cioni G. Electroencephalographic dysmaturity in preterm infants: a prognostic tool in the early postnatal period. Neuropediatrics 1996;27(6):311-6.

62. Scher MS. Automated EEG-sleep analyses and neonatal neurointensive care. Sleep Med 2004;5(6):533-40.

63. Scher MS. Neurophysiological assessment of brain function and maturation. II. A measure of brain dysmaturity in healthy preterm neonates. Pediatr Neurol 1997;16(4):287-95.

64. Scher MS. Neurophysiological assessment of brain function and maturation: I. A measure of brain adaptation in high risk infants. Pediatr Neurol 1997;16(3):191-8.

65. Lommen CM, Pasman JW, van Kranen VH, Andriessen P, Cluitmans PJ, van Rooij LG, et al. An algorithm for the automatic detection of seizures in neonatal amplitude-integrated EEG. Acta Paediatr 2007;96(5):674-80.

66. Niemarkt HJ, Andriessen P, Peters CH, Pasman JW, Bambang Oetomo S. Maturational changes in EEG activity in very preterm infants by analysis of fluctuations in burst suppresion ratio. Acta Paediatr 2007(96 (supplement 456)):674-680.

67. Niemarkt HJ, Andriessen P, Peters CH, Pasman JW, Bambang Oetomo S. Maturational changes in the amplitude-integrated EEG (aEEG) background pattern of preterm infants: a quantitative approach. Acta Paediatr $2007(96$ (supplement 456)):144.

68. Connell JA, Oozeer R, Dubowitz V. Continuous 4-channel EEG monitoring: a guide to interpretation, with normal values, in preterm infants. Neuropediatrics 1987;18(3):138-45.

69. Van Sweden B, Koenderink M, Windau G, Van de Bor M, Van Bel F, Van Dijk JG, et al.
Long-term EEG monitoring in the early premature: developmental and chronobiological aspects. Electroencephalogr Clin Neurophysiol 1991;79(2):94-100.

70. Goto K, Wakayama K, Sonoda H, Ogawa T. Sequential changes in electroencephalogram continuity in very premature infants. Electroencephalogr Clin Neurophysiol 1992;82(3):197-202.

71. Hayakawa M, Okumura A, Hayakawa F, Watanabe K, Ohshiro M, Kato Y, et al. Background electroencephalographic (EEG) activities of very preterm infants born at less than 27 weeks gestation: a study on the degree of continuity. Arch Dis Child Fetal Neonatal Ed 2001;84(3):F163-7.

72. Eyre JA, Nanei S, Wilkinson AR. Quantification of changes in normal neonatal EEGs with gestation from continuous five-day recordings. Dev Med Child Neurol 1988;30(5):599-607.

73. Bell AH, Greisen G, Pryds O. Comparison of the effects of phenobarbitone and morphine administration on EEG activity in preterm babies. Acta Paediatr 1993;82(1):35-9.

74. Stockard-Pope JE, Werner SS, Bickford R. Ontogenesis of EEG in Premature Infants. In: Stockard-Pope JE, Werner SS, Bickford R, editors. Atlas of Neonatal Electroencephalography. New York: Raven Press; 2006. p. 105-175. 



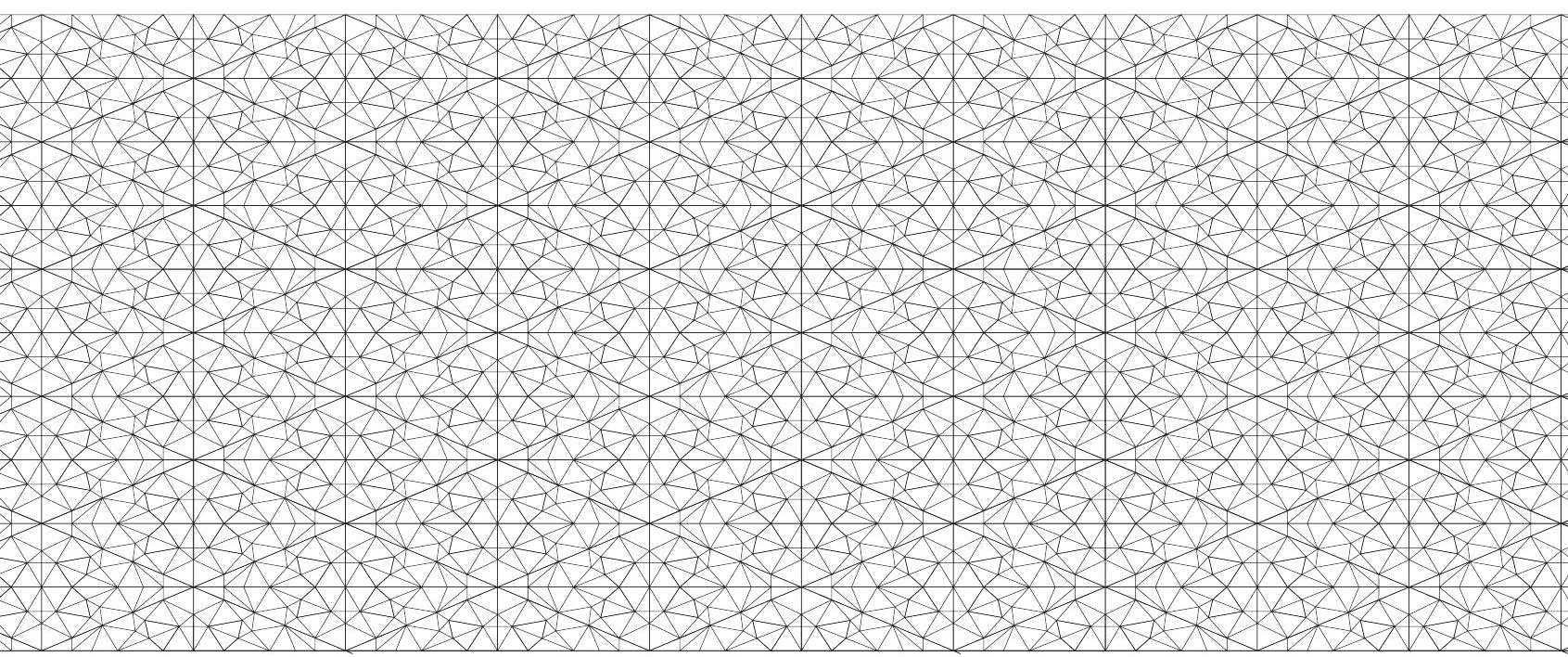




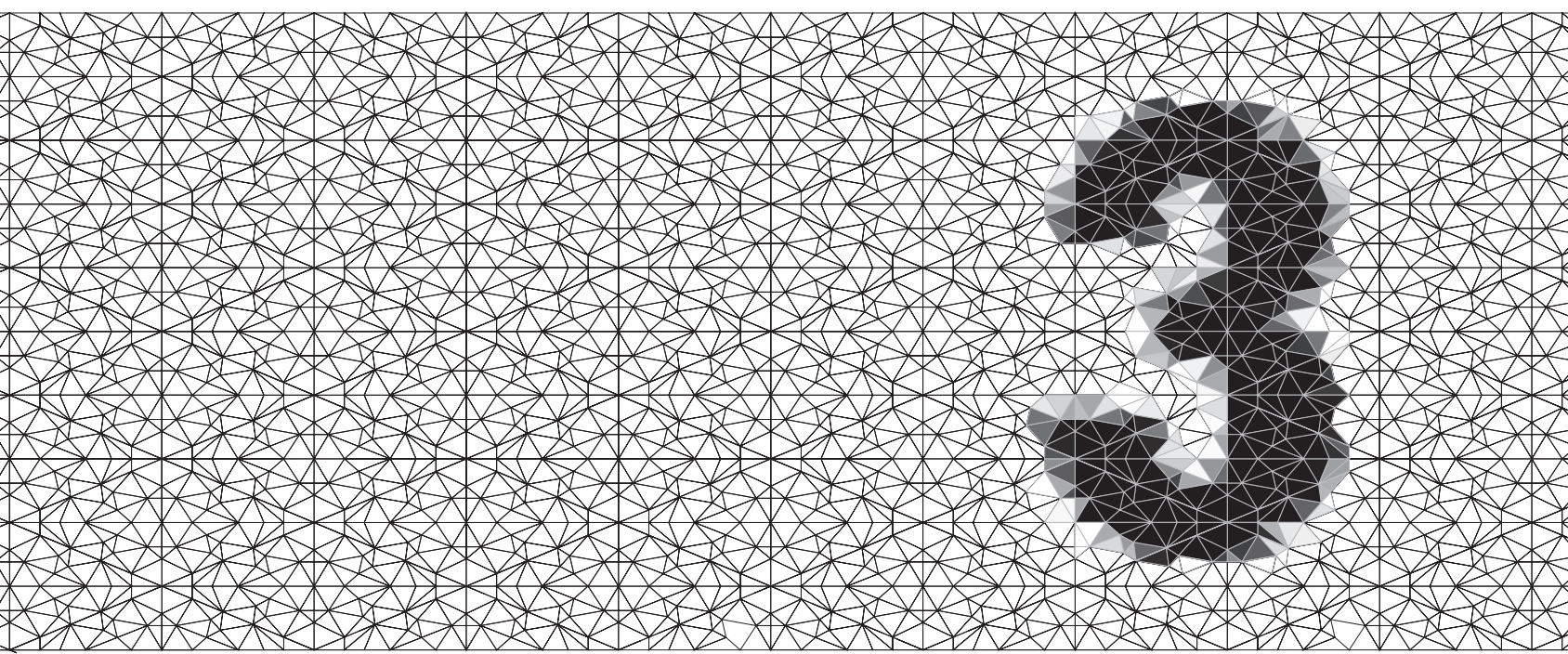

\title{
Quantitative analysis of maturational changes in EEG background activity in very preterm infants with a normal neurodevelopment at 1 year of age
}

\author{
Hendrik J. Niemarkt \\ Peter Andriessen \\ Chris H.L. Peters \\ Jaco W. Pasman \\ Luc J. Zimmermann
}

Sidarto Bambang Oetomo

Early Hum Dev. 2010 Apr;86(4):219-24. 


\section{Abstract}

\section{Background}

The electroencephalographic (EEG) background pattern of preterm infants changes with postmenstrual age (PMA) from discontinuous activity to continuous activity. However, changes in discontinuity have been investigated by visual analysis only.

\section{Aim}

To investigate the maturational changes in EEG discontinuity in healthy preterm infants using an automated EEG detection algorithm.

\section{Study design}

Weekly $4 \mathrm{~h}$ EEG recordings were performed in preterm infants with a gestational age (GA) between $<32$ weeks and normal neurological follow-up at 1 year. The channel $\mathrm{C}_{3}-\mathrm{C}_{4}$ was analyzed using an algorithm which automatically detects periods of EEG inactivity (interburst intervals). The interburst-burst ratio (IBR) percentage of EEG inactivity during a moving time window of $600 \mathrm{~s}$ ) and mean length of the interburst intervals were calculated. Using the IBR, discontinuous background activity (periods with high IBR) and continuous background activity (periods with low IBR) were automatically detected and their mean length during each recording was calculated. Data were analyzed with regression and multivariate analysis.

\section{Results}

79 recordings were performed in 18 infants. All recordings showed a cyclical pattern in EEG discontinuity. With advancing PMA, IBR $\left(R_{2}=0.64 ; p<0.001\right)$, interburst interval length $\left(R_{2}=0.43\right.$; $p<0.001)$ and length of discontinuous activity $\left(R_{2}=0.38 ; p<0.001\right)$ decreased, while continuous activity increased $\left(R_{2}=0.50 ; p<0.001\right)$. Multivariate analysis showed that all EEG discontinuity parameters were equally influenced by GA and postnatal age.

\section{Conclusion}

Analyzing EEG background activity in preterm infants is feasible with an automated algorithm and shows maturational changes of several EEG derived parameters. The cyclical pattern in IBR suggests brain organization in preterm infant. 


\section{Introduction}

The electroencephalographic (EEG) patterns of preterm infants are known to change as a function of postmenstrual age (PMA) [1, 2]. An important aspect of EEG maturation is the development in EEG background activity. In preterm infants with PMA $<34$ weeks the EEG consists of mainly two background patterns. First, tracé discontinue, in which bursts of EEG activity are alternated with tracing of low or no EEG activity (interburst intervals). Second, tracé continue, comprising continuous EEG activity without marked frequency or voltage changes in time and space [3-5].

Discontinuity has been investigated in different manners. The percentage of discontinuous tracings in an EEG recording has been assessed on visual recognition [6-8]. Other investigators measured the lengths of burst and interburst intervals, which were also detected by visual recognition of a neurophysiologist [3, 9-11].

Thus, investigating discontinuity with this visual approach may lead to some methodological issues. For example, exact definitions of discontinuous tracings, burst and IBI vary between studies. Besides, EEG analysis by a neurophysiologist is very time consuming and therefore only short EEG epochs were investigated. Other methodological issues concerning some of these studies are the cross-sectional design and inclusion of infants who suffer from neurological morbidity (like intra-ventricular hemorrhages) [9, 12], receive EEG depressing medication, like Phenobarbital [9] or have an adverse outcome [3]. These methodological issues may explain the observed variation in and between studies.

Modern digital EEG recording devices provide the opportunity to assess EEG recordings more quantitatively, which may lead to a more accurate description of preterm EEG background development. The objective of this study is to assess the maturational changes in EEG background activity in a longitudinal study of a cohort of healthy very preterm infants with normal follow up examination at 1 year, using an automated suppressed EEG activity detection algorithm: the interburst burst ratio (IBR).

\section{Methods}

\section{Study subjects}

The study was conducted in the NICU of Máxima Medical Centre in Veldhoven, The Netherlands during the period from May 2006 to July 2007. The hospital's ethics committee approved the study. Infants were enrolled after written informed consent from both parents.

During the study period 449 infants were admitted to our NICU. Of these, 95 infants were eligible for the study, based on 'first-day' inclusion criteria: preterm infants with a GA $<32$ weeks, birth weight appropriate for gestational age, Apgar score $\geq 6$ at $5 \mathrm{~min}$ and arterial umbilical $\mathrm{pH}$ $>7.00$, normal cerebral ultrasonography (intraventricular hemorrhage grade $\leq$ I) [13] and infants who were expected to stay longer than 2 weeks in our hospital.

Of the eligible population, 67 subjects were subsequently excluded in the first week because of cardiovascular instability, requiring volume expansion and/or inotropic medication, abnormalities on cranial ultrasound (intraventricular hemorrhage II-IV and all grades of periventricular leucomalacia) 
Table 1: Characteristics of study population $(n=18)$.

\section{Clinical characteristics}

\begin{tabular}{ll}
\hline Gestational age (wk) & $29.0 \pm 0,3$ \\
\hline Birth weight (g) & $1297 \pm 58$ \\
\hline Female rate (\%) & $39.5 \pm 1.3$ \\
\hline 5-min Apgar score & $7.3 \pm 0.1$ \\
\hline Umbilical arterial pH & 83 \\
\hline Tocolysis (\%) & 94 \\
\hline Antenatal corticoids (\%) & 28 \\
\hline Caesarean section (\%) & 1 (0-2) \\
\hline CRIB score (median and range) & $104 \pm 12$ \\
\hline Mental Bayley score at 12-m & $95 \pm 10$ \\
\hline Motor Bayley score at 12-m & Legend: CRIB, clinical risk index for babies; values expressed as mean \pm SD, percentage or median (range). \\
\hline \hline
\end{tabular}

[14] use of any sedative or anti-epileptic medication, subjects with recurrent apnea treated with doxapram, or no parental consent.

Thus, 28 infants we enrolled in the study. Of these, 10 subjects were excluded from analysis because they met exclusion criteria after the first week $(n=6)$, developed chronic lung disease $(n=2$, defined as the need for supplemental oxygen/ventilation at 36 weeks PMA) [15] or showed mental or motor Bayley Infant Developmental Scores $<85$ at 12 months of age $(n=2)$ [16].

Finally, 18 clinically stable preterm infants with at least 4 EEG recordings and a normal neurological follow-up at 12 month of age were analyzed in this paper. The characteristics of these subjects are shown in table 1.

Cranial ultrasonography was initially performed in all preterm infants in the first three days of life. Thereafter, studies were performed weekly until 1 month of age and repeated at term. Two of the subjects showed signs of germinal matrix hemorrhage, in two subjects transient flaring was observed only in the first week. All subjects showed normal indexes for the ventricles. All infants received caffeine at midnight with serum levels within therapeutic range. The clinical risk index for babies (CRIB score) was used to determine illness severity in the study population[17]. This score was obtained daily during hospital admission and the maximum score of the patient was noted. Infants who were enrolled were followed in our centre at 6 and 12 months of corrected age, using the Bayley Scales of Infant Developmental for mental and motor function at 12 month of age.

\section{Data acquisition}

Starting at the end of the first week of life, weekly 24-channel digital EEG recordings (NicoletOne; Viasys Healthcare, Conshohocken, PA, USA) were performed. Each recording was classified into the following age terminology during the perinatal period: gestational age (GA), postnatal age 
(PA), and postmenstrual age (PMA, obtained by adding GA and PA). After skin preparation (Nuprep Gel, D.O. Weaver, Aurora, CO, USA) Ag/AgCl cup electrodes, filled with a conductive paste (Ten2o, D.O. Weaver, Aurora, CO, USA), were placed according to the international 10-20 reduced montage system [18]. Impedance was kept below $10 \mathrm{k} \Omega$. All recordings took place from 8 to $12 \mathrm{am}$. During the 4 -h recording the infants were in prone or side position. Feeding and care for the infant was carried out according the normal routine of the NICU.

\section{Data analysis}

The raw EEG signal of the central channel ( $\left.\mathrm{C}_{3}-\mathrm{C}_{4}\right)$ was analyzed with a so-called 'burst-suppression' algorithm, provided by the NicoletOne monitor.

After filtering the EEG signal below 0.5 and above $8 \mathrm{~Hz}$, the algorithm identifies the beginning of an interburst interval when EEG power is below a power threshold of $40 \mu \mathrm{V}^{2}$. The end of the interburst interval is defined when the EEG power rises above a power threshold of $300 \mu \mathrm{V}^{2}$. These power thresholds correspond with a peak-wave amplitude of approximately $10 \mu \mathrm{V}$ and 25 $\mu \mathrm{V}$ respectively and are in agreement with the definition for interburst interval as mentioned by other authors $[1,10,19]$.

Subsequently, the algorithm calculates an interburst-burst ratio (IBR), which is defined as the percentage of time the EEG signal is in a suppression state (i.e. interburst interval) in a 600-s moving window. Thus, if the IBR value is $90 \%$, then the signal is in a suppression state (i.e. interburst interval) $90 \%$ of the time. In this way, high IBR represents discontinuous activity. On the other hand, low IBR values represent continuous activity.

The software application of the NicoletOne monitor calculates a mean length of interburst interval and IBR value every $15 \mathrm{~s}$.

Most artifacts are high frequent and short in duration. As the IBR algorithm filters frequencies above $8 \mathrm{~Hz}$ and calculates a mean over a fairly long time-window of $600 \mathrm{~s} \mathrm{IBR}$ data is not supposed to be greatly influenced by artifacts. Only artifacts related to repositioning the infant or replacing an electrode when impedance was above $10 \mathrm{k} \Omega$, were removed. This was less than $5 \%$ of the total data.

The IBR data were first analyzed visually. IBR is not a constant factor but fluctuates in time: periods with continuous EEG activity (low IBR values) are alternated with tracing of discontinuous activity (high IBR values) (figure 1). An algorithm was developed to split periods of relatively high IBR values from periods with low IBR values. The algorithm automatically segregated periods with high IBR values from periods with low IBR values using an arbitrary calculated threshold value, equivalent to $20^{\text {th }}$ percentile of IBR values. In this way we attempted to segregate the tracing into discontinuous (IBR $>2 \mathrm{O}^{\text {th }}$ percentile) and continuous activity (IBR $<2 \mathrm{O}^{\text {th }}$ percentile).

Thus, from each 4-h EEG recording, mean IBR, total length of discontinuous activity (IBR $>20^{\text {th }}$ percentile), total length of continuous activity (IBR $<20^{\text {th }}$ percentile), and mean interburst interval length during discontinuous activity were calculated.

\section{Statistical analysis}

Data were statistically analyzed with SPSS 13.1 (Inc, Chicago, IL, USA). The Pearson correlation coefficient $r$ was used to evaluate the correlation between age parameters (GA, PA, and PMA) and EEG discontinuity parameters (IBR, length of discontinuous activity, length of continuous activity 
Figure 1: From top to bottom: four $\mathrm{C} 3-\mathrm{C} 4$ consecutive recordings in a preterm infant with a gestational age of 28 weeks at the end of the first (postmenstrual age, PMA, 29 weeks), second (PMA, 30 weeks), third (PMA, 31 weeks) and fourth week (PMA, 32 weeks) after birth.

The $\mathrm{Y}$-axis shows the IBR values (expressed in percentage) as function of recording time (expressed in minutes). Note that even in the very preterm infant the IBR value changes periodically. The shaded areas are automated determined periods with IBR values > empirical threshold value, indicating discontinuous activity. The remaining (white) areas indicate continuous activity. With postmenstrual age, continuous activity increases.

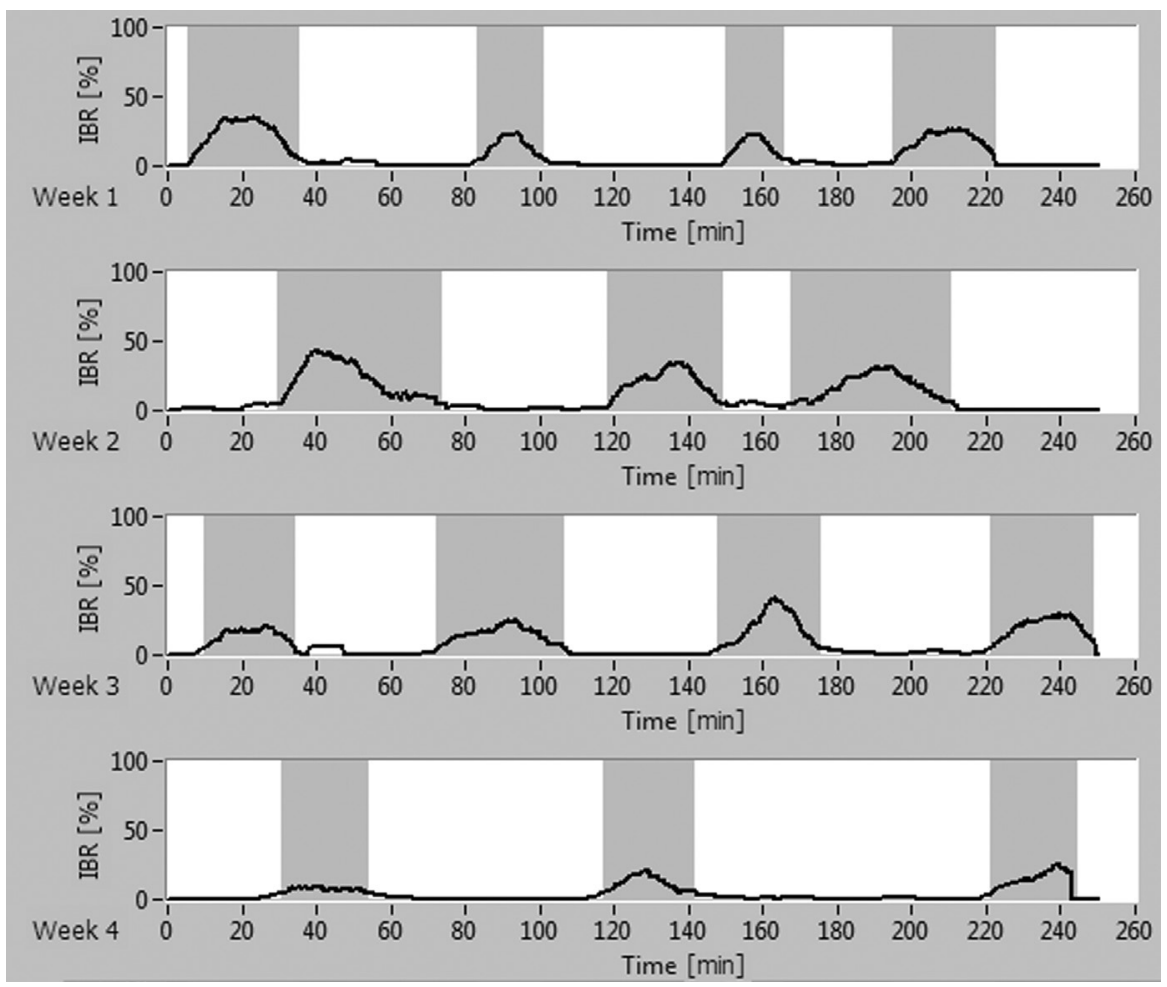

and interburst interval length). In addition, a multivariate analysis was performed to study the influence of GA and PA on the quantitative EEG parameters. To analyze the influence of PMA on EEG parameters, linear regression analysis was performed. Data was assessed on linearity. If there was non-linearity, log transformation was applied before linear regression analysis. Multivariate and linear-regression analysis results are shown with $\mathrm{R}^{2}$ and $\beta$ coefficients with $95 \%$ confidence intervals. Collinearity was studied by the Variance Inflation Factor (VIF), which measures the impact of collinearity among the variables in a regression model. VIF values that exceed 10 are regarded as indicating multicollinearity. A p-value $<0.05$ was considered statistically significant. 


\section{Results}

Eighteen clinically stable infants (GA $29.0 \pm 0.3$ week; birth weight $1297 \pm 58$ g; median CRIB-score 1) were studied and underwent four to six weekly EEG recordings ( 4 recordings in $n=13$ infants; 5 recordings in $n=3$ infants and 6 recordings in $n=2$ infants). All EEG recordings $(n=79)$ represented a large variability in PMA, ranging from 28 to 36 weeks. See table 1 for details of the study population.

In nearly all of the recordings, IBR showed a cyclic pattern of alternating discontinuous EEG activity (high IBR) and continuous activity (low IBR) (figure 1).

Eighteen recordings, performed in the first week of life, were studied to evaluate the effect of GA on the automated derived EEG parameters. Table 2 shows the Pearson correlation coefficients between GA and quantitative parameters (mean IBR, length discontinuous activity, length continuous activity, interburst length). With advancing GA, mean IBR decreased significantly ( $r=$ $-0.63 ; p<0.01)$. The length of continuous activity increased $(r=+0.62 ; p<0.01)$, while the length of the discontinuous activity decreased $(r=-0.60 ; p<0.05)$. Length of interburst interval decreased with GA $(r=-0.65 ; p<0.01)$.

The postnatal changes in quantitative EEG parameters were studied longitudinally in 18 individual subjects with normal postnatal clinical course and normal follow-up at one year (table 2). The correlations between EEG parameters and PA were similar to the correlations between the EEG parameters and GA.

In 16 of the 18 infants (89\%), mean IBR decreased. The length of discontinuous activity decreased and length of continuous activity increased in $15(83 \%)$ and 16 (89\%) patients, respectively. Interburst interval length decreased in $15(83 \%)$ infants.

To investigate whether GA or PA influenced EEG continuity multivariate analysis was performed, with the EEG parameters as dependent. As $\beta$-coefficients of PA and GA were similar, EEG parameters were equally influenced by PA as GA (table 3). As we observed no significant differences between the influence of PA and GA on the EEG parameters, the relation between the automated derived EEG parameters and PMA (GA+PA) was investigated.

As the relationship between PMA and all calculated parameters - except for interburst length - was not linear, log transformation was applied for IBR, length of discontinuity and continuous activity. Mean IBR decreased exponentially with PMA ( $\left.\log I B R=5.5-1.4 \times P M A ; R^{2}=0.64 ; p<0.001\right)$. The length of discontinuous activity exponentially decreased $\left(R^{2}=0.38 ; p<0.001\right)$ while the length of continuous activity exponentially increased $\left(R^{2}=0.50 ; p<0.001\right)$. There was a linear decrease in interburst length $\left(R^{2}=0.43 ; p<0.001\right)$ (figure 2 ).

As mean IBR is influenced by the length of discontinuous activity, continuous activity and interburst length, multivariate analysis was performed to analyze the influence of these parameters on IBR. All three parameters influenced independently mean IBR, with IBI length as most important parameter (table 4). 
Table 2: Correlation between automated derived EEG parameters and gestational age (GA) and postnatal age $(\mathrm{PA})$, respectively.

\begin{tabular}{lll} 
Pearson correlation coefficient & GA $(\mathbf{n}=18)$ & PA $(\mathbf{n}=79)$ \\
\hline mean IBR & $-0.63^{*}$ & $-0.52^{*}$ \\
length continuous activity & $-0.60^{*}$ & $-0.43^{*}$ \\
length discontinuous activity & $+0.62^{*}$ & $+0.44^{*}$ \\
interburst length & $-0.65^{*}$ & $-0.45^{*}$ \\
\hline
\end{tabular}

Legend: Correlation between the automated derived EEG parameters and gestational age (GA, 18 EEG recordings performed at the end of the first week) and postnatal age (PA, comprising all 79 recordings). Interburst burst ratio (IBR) *p value $<0.01$

Table 3: Multivariate analysis model.

\begin{tabular}{|c|c|c|c|}
\hline Dependent variables & $\ddot{R^{2}}$ & $\begin{array}{l}\ldots \ldots \ldots \text { Indepe } \\
\beta \text { B coefficient GA }\end{array}$ & $\begin{array}{l}\text { ables } \\
\text { …........... }\end{array}$ \\
\hline mean IBR & 0.52 & $-4.1[-5.4 ;-2.9]^{*}$ & $-4.2[5.3 ;-3.0]^{*}$ \\
\hline length discontinuous activity & 0.32 & $-4.7[-6.6 ;-2.7]^{*}$ & $-4.4[-6.1 ;-2.6]^{*}$ \\
\hline length continuous activity & 0.41 & $+9.9[+6.3 ;+13.6]^{*}$ & $+9.8[+6.3 ;+13.2]^{*}$ \\
\hline interburst length & 0.46 & $-0.48[-0.64 ;-0.32]^{*}$ & $-0.45[-0.59 ;-0.31]^{*}$ \\
\hline \multicolumn{4}{|c|}{$\begin{array}{l}\text { Legend: Pooled data of all } 79 \text { recordings were used to study the contribution of gestational age (GA) and postnatal age (PA) } \\
\text { on automated derived EEG parameters. Multivariate analysis model with GA and PA as independents variables ( } \beta \text { coefficient } \\
\text { expressed as mean and } 95 \% \text { confidence interval) and EEG characteristics as dependent outcome variables. } \\
{ }^{*} \text { p-value }<0.01\end{array}$} \\
\hline
\end{tabular}

Table 4: Multivariate model

\begin{tabular}{|c|c|c|}
\hline Independent & Standardized $\beta$ coefficient & VIF value \\
\hline Length discontinuous activity & $+0.18^{*}$ & 1.53 \\
\hline Length continuous activity & $-0.15^{*}$ & 1.37 \\
\hline interburst interval length & $+0.68^{*}$ & 1.64 \\
\hline \multicolumn{3}{|c|}{$\begin{array}{l}\text { Legend: A multivariate model was used to investigate the influence of discontinuous activity, continuous activity and } \\
\text { interburst interval on mean value of interburst burst ratio (IBR). All three parameters influenced mean BIR independently, } \\
\text { with mean interburst interval length as most important factor } \\
\text { Collinearity was studied by the Variance Inflation Factor (VIF value), which measures the impact of collinearity among the } \\
\text { variables in a regression model. VIF values }<10 \text { indicate that multicollinearity is not very likely. } \\
{ }^{*} \text { p-value }<0.01\end{array}$} \\
\hline
\end{tabular}




\section{Discussion}

To our knowledge, this is the first study where maturational changes in EEG background activity were studied by automated quantitative analysis of EEG's in very preterm infants with normal neurodevelopment at the age of one year. We observed periodic cycling of IBR in the first week after birth even in very preterm infants. IBR, length of discontinuous and continuous activity and interburst interval length correlated with PA and GA, respectively. These findings were consistent in the study population. When analyzing the development of these parameters longitudinally, a decrease in mean IBR and mean IBI length was found in 16 and 15 of the 18 infants, respectively.

Figure 2: 79 recordings, performed in 18 individual infants with 4-6 weekly recordings were used to study the effect of postmenstrual age (PMA) on four quantitative EEG parameters. a) Mean interburst burst ratio (IBR) decreased exponentially with PMA $\left(R^{2}=0.64 ; p<0.001\right)$. b) Interburst interval length showed a linear decrease with PMA $\left(R^{2}=0.43 ; p<0.001\right)$. c) Length of discontinuous activity exponentially decreased $\left(R^{2}=0.38 ; p<0.001\right)$ and d) Length of continuous activity exponentially increased $\left(R^{2}=0.50 ; p<0.001\right)$ with $P M A$.
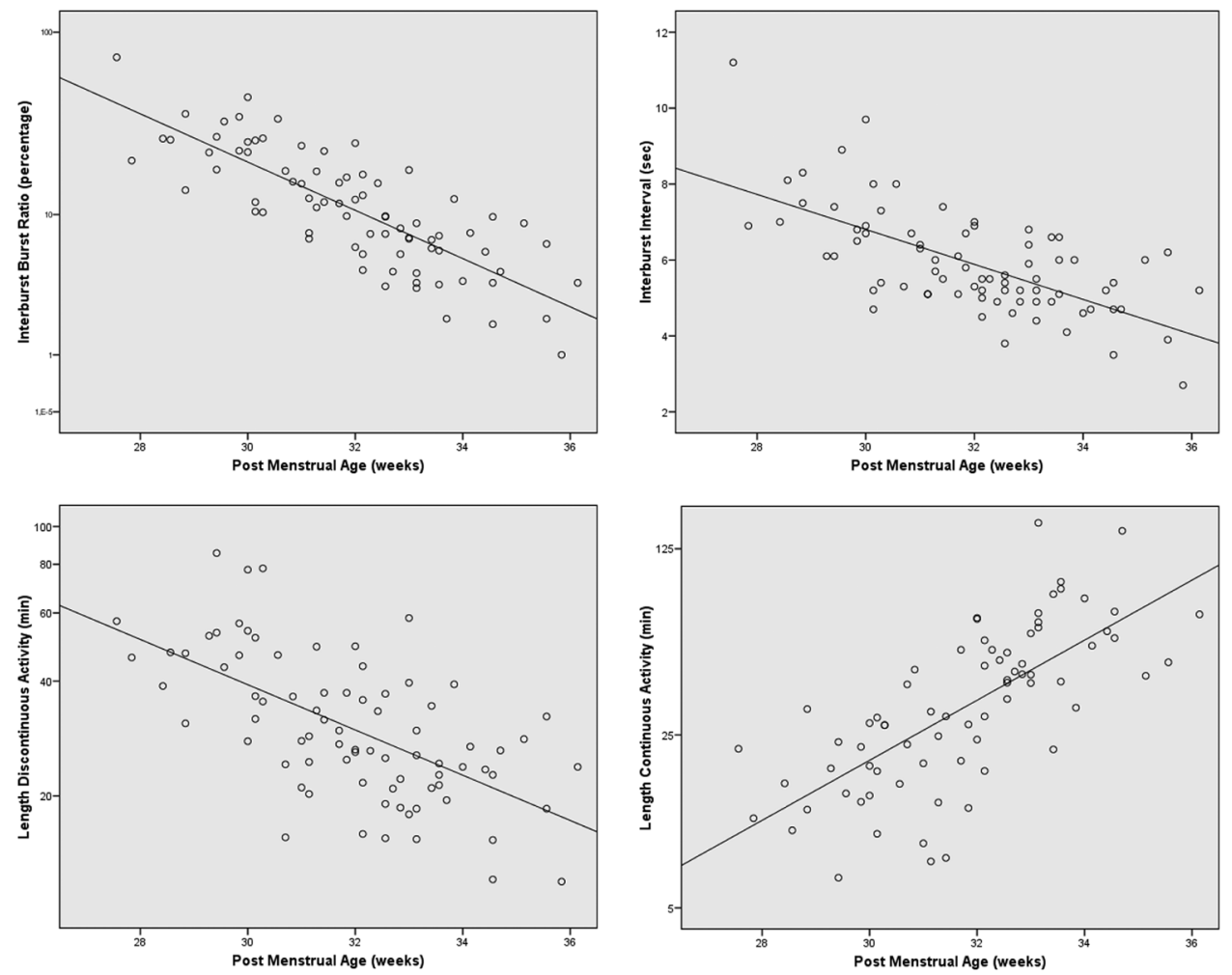

\section{Rest-activity cycling}

The IBR showed periodic variations within a 4 -h recording, even in very preterm infants. This confirms the concept of rest-activity cycles or rudimentary sleep-wake cycles in very preterm 
infants $[20,21]$. The absence of sleep-wake cycling in amplitude integrated EEG (aEEG) recordings in term asphyxiated infants is related to adverse neurological outcome [22]. If the absence of the rest-activity cycles in very preterm infants is a sign of pathology remains unclear. However, preterm infants with severe intra-ventricular hemorrhages do not show signs of sleep-wake cycles [5, 23]. Thus, the observation of rest- activity cycles indicates physiologic brain organization.

\section{Changes in discontinuity}

Overall discontinuity, as measured by mean IBR, decreased exponentially with increasing PMA. Two major changes in discontinuity were observed.

First, the length of discontinuous activity decreased exponentially with increasing PMA, with the highest decrease before 32 weeks PMA. This was also observed by others $[3,7,8,11]$. Conversely, the length of the continuous activity increases exponentially.

Second, within the periods of discontinuous activity, there were shorter periods of suppressed EEG activity. This is reflected in the decrease in length of the IBI intervals. This decrease was in a linear fashion and was also observed by other authors [9-11, 24]. However, the observed interburst interval lengths in our study were within a much smaller range, which may be explained by the automatic detection method and the strict inclusion criteria. Another explanation is that we started our recordings one week after birth in contrast to other studies. Recent data shows that continuity increases with $63 \%$ over the first week of life and may be related to the increase in cerebral blood flow during the first days of life [25]. Changes in EEG continuity during the first days post partum may reflect rather the adaptation to extra-uterine life than maturation of the brain. To our best knowledge, the maturational decrease in IBI length in serial EEG recordings of individual infants has not been studied yet.

Of all parameters mean IBR is the best predictor of PMA and represents the overall continuity of an EEG tracing. PMA explains $64 \%$ of the variation in mean IBR (figure 2). Mean IBR is determined by (consecutively) interburst interval length, length of discontinuous activity and length of continuous activity. Thus, the overall change in continuity is rather caused by a decrease in length in interburst intervals within periods of discontinuous activity than a decrease in length of the discontinuous activity itself.

The actual physiological explanation of the changes in continuity is not elucidated yet. It is suggested that the shortening of interburst interval length is related to maturation in excitatory synapses or loss of presumed sub-cortical systems responsible for the abrupt inhibition of cortical activity $[10,24,26]$. It is demonstrated that interburst interval length is related to adverse outcome and abnormalities on cranial ultrasonography. Benda demonstrated in a prospective study that prolonged interburst interval length (>30s) is related to intraventricular haemorrhages and fatal outcome [9]. Hahn, studying infants with GA >26 weeks and normal outcome at 3 years found interburst interval length shorter than $20 \mathrm{~s}$ [10]. Conde observed significantly longer interburst interval length in preterm infants with major ultrasound lesions [19]. To our knowledge, the maturational decrease in interburst interval length has not been studied longitudinally in very preterm infants with a normal neurodevelopment at 12 months. 


\section{Clinical relevance}

As cyclic variations in IBR may be an indication of physiologic brain organization and changes in EEG continuity are related to neurological outcome, an automated algorithm which detects maturational changes in EEG continuity may become useful as a tool to asses brain maturation in the clinical setting.

Indeed, Wikström found longer interburst interval length in a small group of preterm infants with brain injury using an automated interburst interval detection algorithm [27].

Bedside digital amplitude-integrated EEG-recorders are used increasingly in the neonatal intensive care unit. Most of these recorders also provide raw EEG data on which automated EEG continuity analysis can be performed. Automated analysis enables to perform a quantitative analysis in the daily routine of the neonatal intensive care unit. Eventually, bedside EEG devices could not only give information about neonatal convulsions but may also provide information about preterm brain development and identify factors which interfere with normal brain maturation.

\section{Methodological considerations}

The number of studied infants was relatively small. However, the aim of this study was to assess automated derived EEG parameters in a cohort of 'healthy' preterm infants without any additional pathologic conditions that might interfere with normal cerebral development. Therefore we applied strict inclusion and exclusion criteria for the study, resulting in this relatively small population. Of the eligible 95 infants, only 18 were included in the final analysis.

All very preterm infants showed normal neurodevelopment after 1 year of follow-up. Second, interburst intervals are frequently studied in multichannel EEG and in this study we used a single EEG channel ( $\left.\mathrm{C}_{3}-\mathrm{C}_{4}\right)$ for analyzing discontinuity. However, as interburst interval length is mostly defined as EEG inactivity in one or more channels, we assume this one-channel approach is appropriate to describe this aspect. It has been argued that single channel aEEG is not accurate enough to detect localized seizure activity [28]. However, the aim of this study was to describe the overall background electrical activity of the brain and for this purpose single channel measurement can be considered as an adequate method.

\section{Conclusion}

Discontinuity was quantitatively investigated by an automated algorithm in a healthy preterm population with normal follow up at one year. The EEGs showed a cyclic variation in mean IBR, indicating physiologic brain organization. IBR is exponentially correlated with PMA and is in particular determined by a decrease in interburst interval, length of discontinuous activity and an increase in length of continuous tracing. Automated analysis of discontinuity of the preterm brain may become useful to asses brain maturation. 


\section{Acknowledgements}

We would like to thank A. Smets, Department of Clinical Neurophysiology of the Máxima Medical Centre for his help in performing the EEG recordings.

We also would like to thank T. Katgert, Department of Psychology of the Máxima Medical Centre for performing the follow-up examinations in the studied infants. 


\section{References}

1. Niemarkt HJ, Andriessen P, Pasman J, Vles JS, Zimmermann LJ, Bambang Oetomo S. Analyzing EEG maturation in preterm infants: the value of a quantitative approach. J PerinatNeonatal Med 2008;1(3):131-44.

2. Selton D, Andre M, Hascoet JM. Normal EEG in very premature infants: reference criteria. Clin Neurophysiol 2000;111(12):2116-24.

3. Anderson CM, Torres F, Faoro A. The EEG of the early premature. Electroencephalogr Clin Neurophysiol 1985;60(2):95-105.

4. Holmes GL, Lombroso CT. Prognostic value of background patterns in the neonatal EEG. J Clin Neurophysiol 1993;10(3):323-52.

5. Lombroso CT. Neonatal polygraphy in full-term and premature infants: a review of normal and abnormal findings. J Clin Neurophysiol 1985;2(2):105-55.

6. Connell JA, Oozeer R, Dubowitz V. Continuous 4-channel EEG monitoring: a guide to interpretation, with normal values, in preterm infants. Neuropediatrics 1987;18(3):138-45.

7. Goto K, Wakayama K, Sonoda H, Ogawa T. Sequential changes in electroencephalogram continuity in very premature infants. Electroencephalogr Clin Neurophysiol 1992;82(3):197-202.

8. Van Sweden B, Koenderink M, Windau G, Van de Bor M, Van Bel F, Van Dijk JG, et al. Long-term EEG monitoring in the early premature: developmental and chronobiological aspects. Electroencephalogr Clin Neurophysiol 1991;79(2):94-100.

9. Benda Gl, Engel RC, Zhang YP. Prolonged inactive phases during the discontinuous pattern of prematurity in the electroencephalogram of very-low-birthweight infants. Electroencephalogr Clin Neurophysiol 1989;72(3):189-97.

10. Hahn JS, Monyer H, Tharp BR. Interburst interval measurements in the EEGs of premature infants with normal neurological outcome. Electroencephalogr Clin Neurophysiol 1989;73(5):410-8.

11. Hayakawa M, Okumura A, Hayakawa F, Watanabe K, Ohshiro M, Kato Y, et al. Background electroencephalographic (EEG) activities of very preterm infants born at less than 27 weeks gestation: a study on the degree of continuity. Arch Dis Child Fetal Neonatal Ed 2001;84(3):F163-7.

12. Biagioni E, Bartalena L, Boldrini A, Cioni G, Giancola S, Ipata AE. Background EEG activity in preterm infants: correlation of outcome with selected maturational features. Electroencephalogr Clin Neurophysiol 1994;91(3):154-62.

13. Volpe JJ. Neurology of the newborn. 5th Edition. Philadelphia: Elsevier; 2008.

14. de Vries LS, Eken P, Dubowitz LM. The spectrum of leukomalacia using cranial ultrasound. Behav Brain Res 1992;49(1):1-6.

15. Lal MK, Manktelow BN, Draper ES, Field DJ. Chronic lung disease of prematurity and intrauterine growth retardation: a populationbased study. Pediatrics 2003;111(3):483-7.

16. Bayley N. Manual for Bayley Scales of Infant Development. 2nd Edition. San Antonio, Texas: The Psychological Corporation; 1993.

17. The CRIB (clinical risk index for babies) score: $a$ tool for assessing initial neonatal risk and comparing performance of neonatal intensive care units. The International Neonatal Network. Lancet 1993;342(8865):193-8.

18. Tekgul H, Bourgeois BF, Gauvreau K, Bergin AM. Electroencephalography in neonatal seizures: comparison of a reduced and a full 10/20 montage. Pediatr Neurol 2005;32(3):155-61.

19. Conde JR, de Hoyos AL, Martinez ED, Campo CG, Perez AM, Borges AA. Extrauterine life duration and ontogenic EEG parameters in preterm newborns with and without major ultrasound brain lesions. Clin Neurophysiol 
2005;116(12):2796-809.

20. Curzi-Dascalova L, Figueroa JM, Eiselt $M$, Christova E, Virassamy A, d'Allest AM, et al. Sleep state organization in premature infants of less than 35 weeks' gestational age. Pediatr Res 1993;34(5):624-8.

21. Scher MS, Johnson MW, Holditch-Davis D. Cyclicity of neonatal sleep behaviors at 25 to 30 weeks' postconceptional age. Pediatr Res 2005;57(6):879-82.

22. Osredkar D, Toet MC, van Rooij $L G$, van Huffelen AC, Groenendaal F, de Vries LS. Sleepwake cycling on amplitude-integrated electroencephalography in term newborns with hypoxic-ischemic encephalopathy. Pediatrics 2005;115(2):327-32.

23. Dreyfus-Brisac C. Ontogenesis of sleep in human prematures after 32 weeks of conceptional age. Dev Psychobiol 1970;3(2):91-121.

24. Victor S, Appleton RE, Beirne M, Marson AG, Weindling AM. Spectral analysis of electroencephalography in premature newborn infants: normal ranges. Pediatr Res 2005;57(3):336-41.

25. West $C R$, Harding JE, Williams $C E$, Gunning MI, Battin MR. Quantitative electroencephalographic patterns in normal preterm infants over the first week after birth. Early Hum Dev 2006;82(1):43-51.

26. Vecchierini MF, d'Allest AM, Verpillat P. EEG patterns in 10 extreme premature neonates with normal neurological outcome: qualitative and quantitative data. Brain Dev 2003;25(5):330-7.

27. Wikstrom S, Ley D, Hansen-Pupp I, Rosen I, Hellstrom-Westas L. Early amplitude-integrated EEG correlates with cord TNF-alpha and brain injury in very preterm infants. Acta Paediatr 2008;97(7):915-9.

28. Shellhaas RA, Soaita Al, Clancy RR. Sensitivity of amplitude-integrated electroencephalography for neonatal seizure detection. Pediatrics 2007;120(4):770-7. 



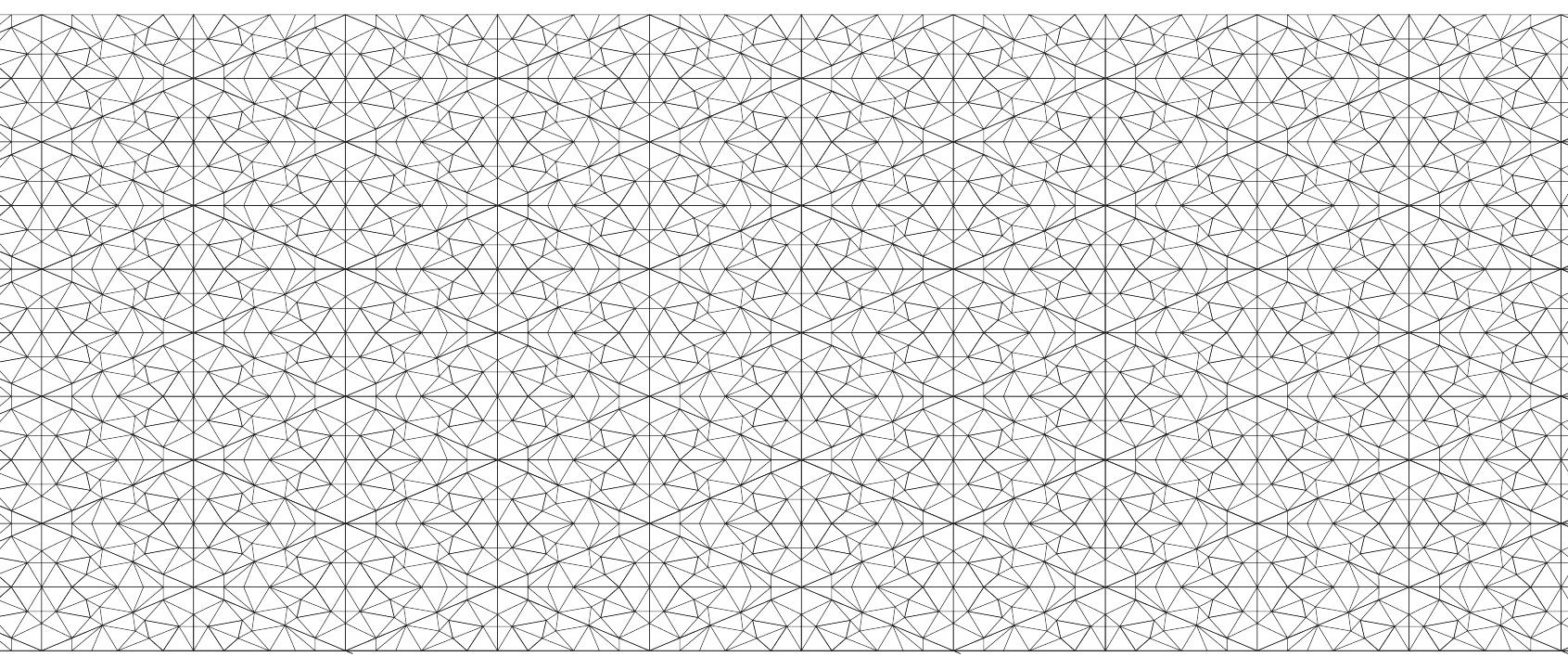




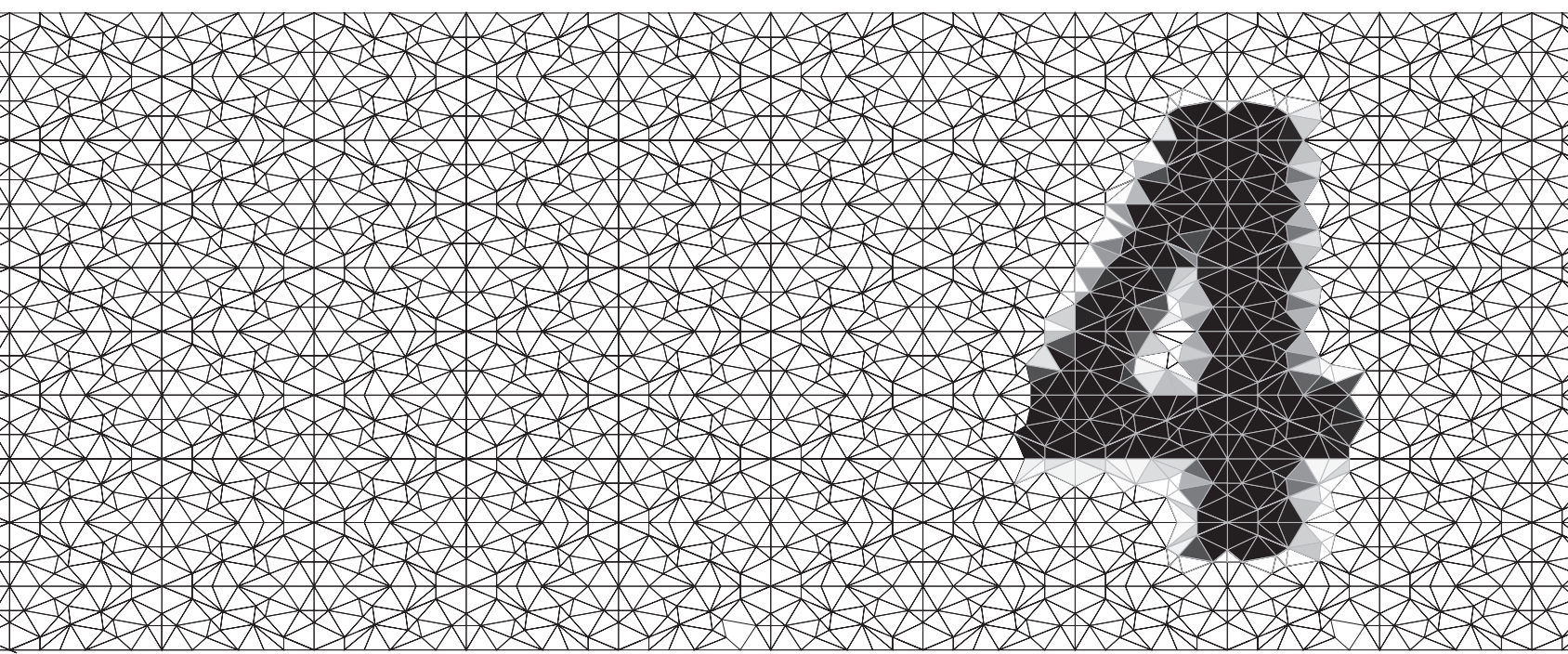

\title{
Automatic burst detection for the EEG of the preterm infant
}

\author{
Ward Jennekens \\ Loes S. Ruijs \\ Charlotte M.L. Lommen \\ Hendrik J. Niemarkt \\ Jaco W. Pasman \\ Vivianne H.J.M. van Kranen-Mastenbroek \\ Pieter F.F. Wijn \\ Carola van Pul \\ Peter Andriessen
}

Physiol Meas. 2011 Oct;32(10):1623-37. 


\section{Abstract}

To aid with prognosis and stratification of clinical treatment for preterm infants, a method for automated detection of bursts, interburst-intervals (IBIs) and continuous patterns in the electroencephalogram (EEG) is developed. Results are evaluated for preterm infants with normal neurological follow-up at 2 years.

The detection algorithm (MATLABR ${ }^{\circledast}$ ) for burst, IBI and continuous pattern is based on selection by amplitude, time span, number of channels and numbers of active electrodes. Annotations of two neurophysiologists were used to determine threshold values. The training set consisted of EEG recordings of four preterm infants with postmenstrual age (PMA, gestational age + postnatal age) of 29-34 weeks. Optimal threshold values were based on overall highest sensitivity. For evaluation, both observers verified detections in an independent dataset of four EEG recordings with comparable PMA. Algorithm performance was assessed by calculation of sensitivity and positive predictive value.

The results of algorithm evaluation are as follows: sensitivity values of $90 \% \pm 6 \%, 80 \% \pm 9 \%$ and $97 \% \pm 5 \%$ for burst, IBI and continuous patterns, respectively. Corresponding positive predictive values were $88 \% \pm 8 \%, 96 \% \pm 3 \%$ and $85 \% \pm 15 \%$, respectively. In conclusion, the algorithm showed high sensitivity and positive predictive values for bursts, IBIs and continuous patterns in preterm EEG. Computer-assisted analysis of EEG may allow objective and reproducible analysis for clinical treatment. 


\section{Introduction}

About $25-40 \%$ of preterm infants suffer from neurobehavioral problems at a later age[1, 2]. The pathogenesis of these problems is often still unclear, though theyare likely related to medical, environmental and iatrogenic conditions interfering with white matter development of the preterm brain [3].

Electroencephalography (EEG) is regarded as the gold standard in the assessment of cerebral function and is one of the most powerful tools for predicting neurological outcome in preterm and term infants $[4,5]$. The EEG can be described in terms of continuity, amplitude, frequency, inter-hemispheric synchrony, and presence of specific maturational patterns and transients [6-8] The progressive development of continuity with advancing postmenstrual age (PMA, gestational + postnatal age) provides the most immediate visually striking aspect of the EEG reflecting brain maturation. The changes in continuity comprise increasing burst duration, decreasing burst amplitude, decreasing length of the interburst intervals(IBIs) and increasing IBI amplitude, resulting in the appearance of continuous patterns $[4,5,7,9-12]$. The variety of these characteristics makes interpretation of tracings very complex. These difficulties of interpretation are increased by absence of agreement about definition of signal properties for quantification. The minimum duration of data segments usable for analysis remains unclear. The amplitude criteria defining discontinuities and the minimum number of electrodes under which EEG activity must be present to be able to reliably identify cerebral activity are other unsolved questions [13]. These differences mean that tracing interpretations vary from one author to another. Studies of cerebral maturation show comparable trends in changes of continuity, but values vary between different studies $[4,9,14-17]$.Other studies have shown that the manifestation of maturation related features during the first days of life is predictive of neurological outcome in preterm infants $[18,19]$. Therefore, a clear description of brain maturation of the cerebral healthy preterm infant is necessary. This could serve as a reference for the determination of pathological conditions that have an adverse influence on brain development, and for studying the effectiveness of possible therapeutic intervention strategies.

For an objective evaluation of electro-cortical maturation, detection algorithms may be very useful for neurophysiologists. Detection of EEG bursts can be performed using various activity measures, e.g. spontaneous signal variance [20] or the nonlinear energy operator [21]. These methods are mostly based on detection from single channel EEG recordings, optimized for longitudinal cerebral monitoring. Varying spatial configuration of EEG transients [22] would favor detection based on multi-channel measurements for in-detail study of maturation. Bursts can also be detected through identification of EEG features yielding optimal sensitivity by using (nonlinear) classifiers, e.g. neuronal networks [23], which were evaluated using data from full-term neonates with perinatal asphyxia. Generalization of classifier results, a nonlinear combination of signal features, from a study population with specific pathology remains difficult.

The aim of this study was to develop a simple method for automated detection of burst, IBI and continuous pattern in the EEG for description of the cerebral development of healthy preterm infants with a normal outcome at 2 years of age. The developed detection method was based on amplitude, duration and number of channels in which the electro-cortical activity is simultaneously present. First, detection parameter threshold values were determined using a training dataset. 
Second, the algorithm was evaluated using an independent dataset.

\section{Methods}

\section{Study subjects}

The EEG data were obtained from a comprehensive EEG research program conducted in the NICU of Máxima Medical Centre in Veldhoven, The Netherlands, during the period from August 2006 to June 2007. The study was approved by the hospital's ethics committee. Infants were enrolled after written informed consent from both parents. For details of the study group we refer to previous papers [24, 25]. In short, inclusioncriteria were gestational age $(G A)<30$ weeks, birth weight appropriate for $G A$, Apgar score $\geq 6$ at $5 \mathrm{~min}$ and arterial umbilical $\mathrm{pH}>7.00$, normal cerebral ultrasonography (no IVH grade2 or higher, no PVL), no congenital malformations, stable respiratory and cardiocirculatory conditions and absence of neurological medication (e.g. Fenobarbital, Midazolam, Morfine, Dopram) within $48 \mathrm{~h}$ from the recording. Infants showed normal neurological follow-up at 2 years of age (mental and motor BSID-II-NL > 85) [26]. For this study eight preterm infants were recruited with a PMA of 29-34 weeks. EEG recordings of four infants were used for the training set and four EEG recordings of infants with comparable PMA were used for the algorithm evaluation.

\section{Data acquisition}

Starting at the end of the first week of life, weekly digital EEG recordings (NicoletOne; Viasys Healthcare, Conshohocken, PA, USA) were performed. After skin preparation (Nuprep Gel, DO Weaver, Aurora, CO, USA), Ag/AgCl cup electrodes filled with conductive paste (Ten2o, DO Weaver, Aurora, CO, USA) were placed according to the international reduced 10-20 montage system [27], i.e. using $\mathrm{Fp}_{2}, \mathrm{Fp}_{1}, \mathrm{C}_{2}, \mathrm{C}_{3}, \mathrm{C}_{4}, \mathrm{~T}_{3}, \mathrm{~T}_{4}, \mathrm{O}_{1}$ and $\mathrm{O}_{2}$ electrodes. Impedance of the electrodes was maintained below $10 \mathrm{k} \Omega$. The digital EEG signal was sampled at 256 or $512 \mathrm{~Hz}$. Artifacts related to repositioning the infant or replacement of an electrode when impedance value was greater than $10 \mathrm{k} \Omega$ were removed. All recordings took place between 8-12 am and after feeding, when the infant was asleep in the incubator in prone or side position. The average recording time was $4 \mathrm{~h}$. During the measurements a video recording of the infant was made in order to relate patterns or artifacts in the EEG to the position or state of the infant.

\section{Burst detection}

The detection algorithm for burst, IBI and continuous pattern is based on selection by amplitude, time span, number of channels and numbers of electrodes. The algorithm has been written in the mathematical program MATLABR ${ }^{\circledR}$ (The MathWorks, MA, USA).

First, the EEG data are imported in Matlab and are filtered using fourth-order Butterworth filters. A high-pass filter with a cut-off frequency of $0.5 \mathrm{~Hz}$ is used for elimination of the dc-component and baseline shifts. A $50 \mathrm{~Hz}$ notch filter is applied to attenuate the interference signal of the mains power supply. The data are further filtered using a fourth order Butterworth low-pass filter at $32 \mathrm{~Hz}$ to remove high frequency artifacts. This filter attenuates high frequency content of the signal that does not correspond to EEG activity, while maintaining the information in the EEG frequency range. 
The next step is the detection of bursts and continuous activity. Based on previous studies[9, 28], we assumed that burst and continuous pattern have the same amplitude threshold and only differ in time span. Therefore, first the burst and the continuous pattern are detected as high-voltage activity. At the end of this procedure the division between burst and continuous pattern is made. The detection of burst and continuous pattern is shown schematically in figure 1.

Envelope values of the signals were determined for application of the amplitude threshold. The envelope values $\mathrm{EV}(i)$ were derived from the average signal power $P(i)$ by

$$
\mathrm{EV}(i)=\sqrt{2 P(i)}=\sqrt{\frac{2}{N_{W}} \sum_{i=1}^{N_{W}} x(i)^{2}},
$$

where $x(i)$ is the amplitude of the signal and NW a window with a length equal to the number of sample points in $1 \mathrm{~s}$ of data.

After calculation of the envelope values for each EEG-channel, a threshold called amplitudethreshold-high (ATH) was applied to all signals. Sample points with an envelope value equal to or above ATH were assigned 1, meaning high-voltage activity. Sample points with an envelope value below ATH were assigned zero.

Using these binary arrays, an overview of the number of channels which simultaneously contain high-voltage activity was obtained. If the number of channels was above a threshold called channelthreshold-high (CTH), the EEG-activity was classified as high-voltage activity.

To determine the onset and offset of burst and continuous pattern, the duration of determined periods of high-voltage activity is elongated to both sides until there is no remaining high-voltage activity in any channel. This is in agreement with the burst length defined by Biagioni [4] and Vecchierini $[4,17]$, where the beginning of the first and the end of the last wave were taken as onset and offset of the burst, respectively. Next, it was assumed that high-voltage activity is followed by periods of low-voltage activity, the IBI $[4,9,17,28]$. Minimum duration of an IBI is defined as $1 \mathrm{~S}$. Therefore, if two detected periods of high-voltage activity appear within $1 \mathrm{~s}$ after each other it is assumed that they belong together and form one period.

Detected segments of high-voltage activity are then checked whether they suffice the defined condition for the number of active electrodes. Since high-voltage activity is most likely an artifact when only one electrode is active, a minimum of two electrodes is required to be active for accurate high-voltage activity detection. If a period could not meet this requirement, the detected segment was rejected.

Finally, periods detected as high-voltage activity are divided into burst and continuous pattern based on the time threshold. Periods $\geq 20 \mathrm{~s}$ are classified as continuous patterns while periods $<20 \mathrm{~s}$ are classified as bursts $[9,28]$. 
Figure 1: Overview of detection procedure for bursts and continuous patterns.

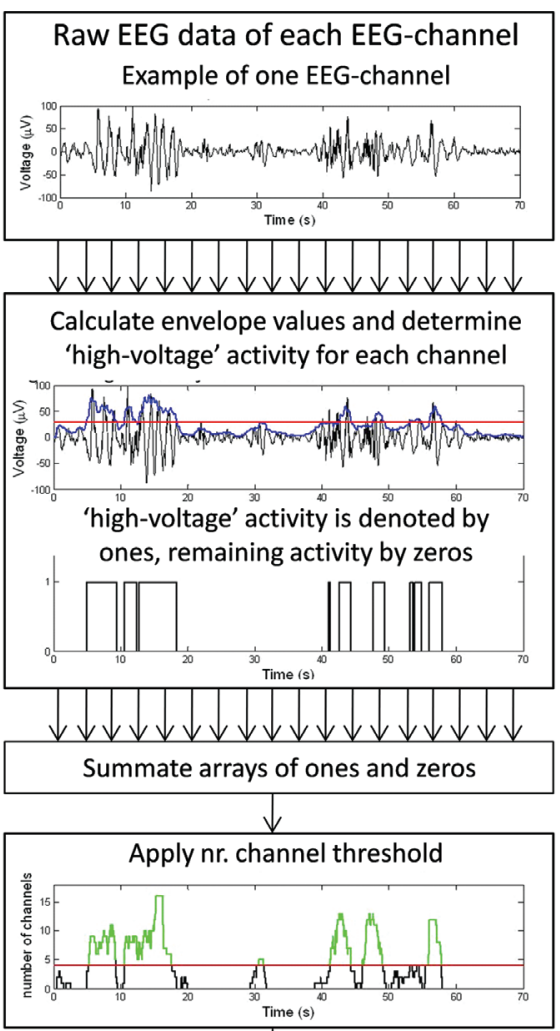

$\downarrow$

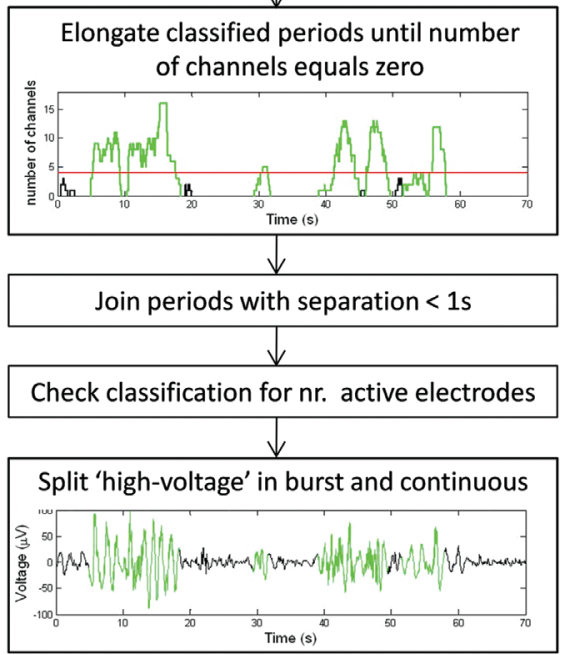

Application of amplitude-threshold (ATH) for each EEG-channel

\author{
From separate EEG-channel \\ analysis to overall EEG
}

\section{Application nr. EEG-channel} threshold (CTH)

\section{Elongation of classified periods}

Check for nr. electrodes threshold

\section{Application of time-threshold}

Legend: Overview of procedure for detection of bursts and continuous patterns. The procedure starts with analysis of each EEG-channel separately. At the end, an overall detection is made for all EEG-channels. 


\section{IBI detection}

The unclassified segments of EEG, between detected bursts and continuous patterns, are analyzed. The detection procedure is comparable to the burst detection; however the sequence of detection steps is different.

First, periods are determined which were not classified as burst or continuous pattern.

Subsequently, the envelope values of these periods are compared with an amplitude-thresholdlow (ATL). If the envelope value is lower than the ATL, the corresponding sample point was assigned a 1, stating a possible IBI. Otherwise a zero was assigned.

By applying a channel-threshold-low (CTL) to the array formed in the preceding step, periods of IBI were determined. Only if the number of channels was above this threshold, the activity was regarded as an IBI. The number of channels threshold value CTL for IBI was set to 18 , corresponding to inactivity of all nine electrodes, based on previous studies[4, 9, 14, 16].

Periods of detected IBI are finally checked for their duration. Because the minimum duration of an IBI had to be at least $1 \mathrm{~S}$, IBI shorter than $1 \mathrm{~s}$ were rejected.

\section{Training}

To determine the threshold values for pattern detection and to check the defined threshold values for consistency, annotations of two clinical neurophysiologists were used. The training set consisted of EEG records obtained from preterm infants of 29, 31, 32 and 34 weeks PMA, respectively. The observers annotated $1 \mathrm{~h}$ of each EEG record. Allowed annotations were burst, IBI, continuous and artifact (specified as muscle, eye, movement or other). The observers could view the video of the infant made during the EEG measurement simultaneously with the EEG record. Overall, annotations were made for $18 \mathrm{EEG}$-channels. Annotated events had a precision of $1 \mathrm{~s}$.

The threshold values of detection parameters regarding time span and number of electrodes remain as defined previously. Threshold values of amplitude (ATH and ATL) and number of EEGchannels $(\mathrm{CTH})$ were defined by determining the most optimal sensitivity value of the algorithm when compared with agreed annotations of the observers. The most optimal values are those threshold values in which the highest sensitivity rates could be reached for all three classifications, i.e. burst, IBI and continuous activity.

\section{Evaluation}

For evaluation of the algorithm the observers verified all detections. Events verified by the observers consisted of $|\mathrm{B}|$, burst, continuous patterns and undefined activity. EEG activity that was not classified as IBI, burst or continuous pattern was classified as undefined activity. For each event, the observers had to indicate whether they could or could not agree with the detection of the algorithm. The observers had to judge the detection as correct when the particular classification was present in the segment detected by the algorithm. The accuracy of the onset or offset of an event was not taken into account. If the observers did not agree with the detection, they had to indicate what type of activity the algorithm should have detected. If events were detected incorrectly due to the presence of artifacts, the observers were asked to specify the type of artifact.

A measure of the performance of the algorithm was obtained by calculation of the sensitivity and the positive predictive value (PPV) for each type of classification. EEG data used for evaluation 
Figure 2: ATH sensitivity of burst, IBI, and continuous pattern detection.
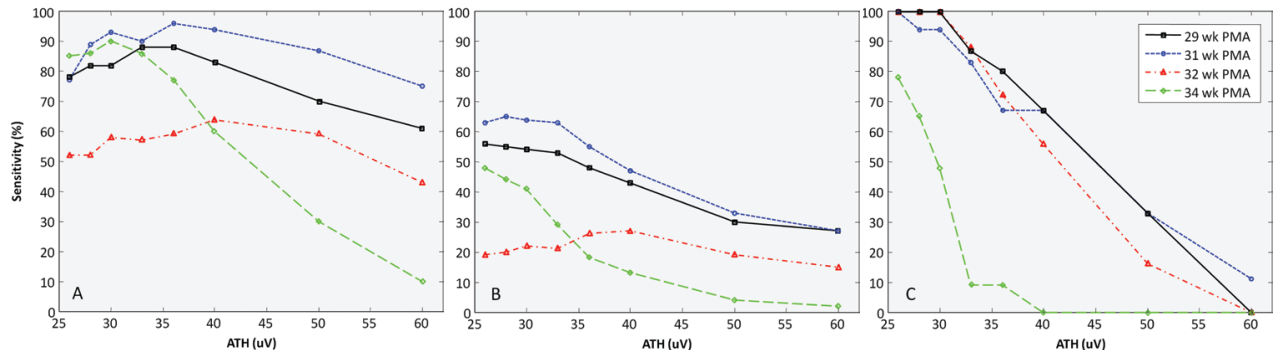

Legend: Sensitivity of detection algorithm for A) burst, B) IBI, and C) continuous pattern after application of different threshold values for amplitude-threshold-high (ATH) for detection of high-voltage-activity.

Figure 3: CTH sensitivity of burst, IBI, and continuous pattern detection.
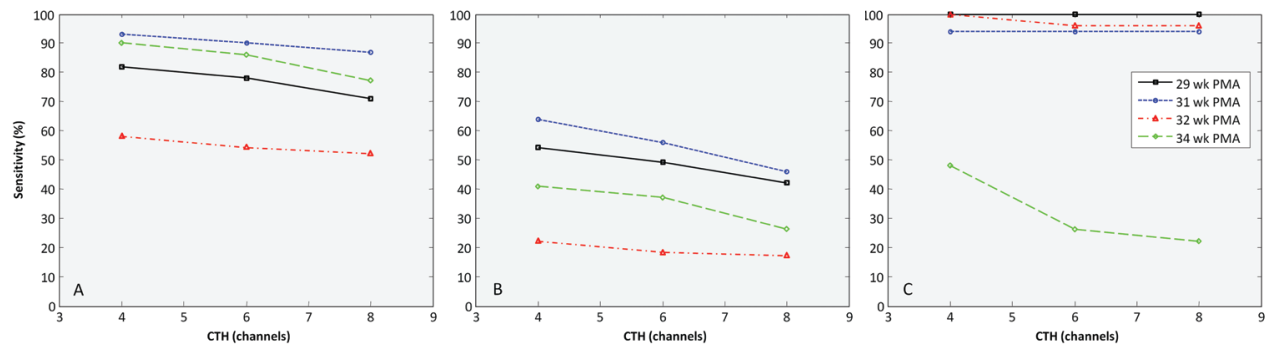

Legend: Resulting sensitivity of detection of A) bursts, B) IBI, and C) continuous patterns after application of different threshold values for number of channels (CTH) for detection of high-voltage-activity.

Figure 4: ATL sensitivity of IBI detection.
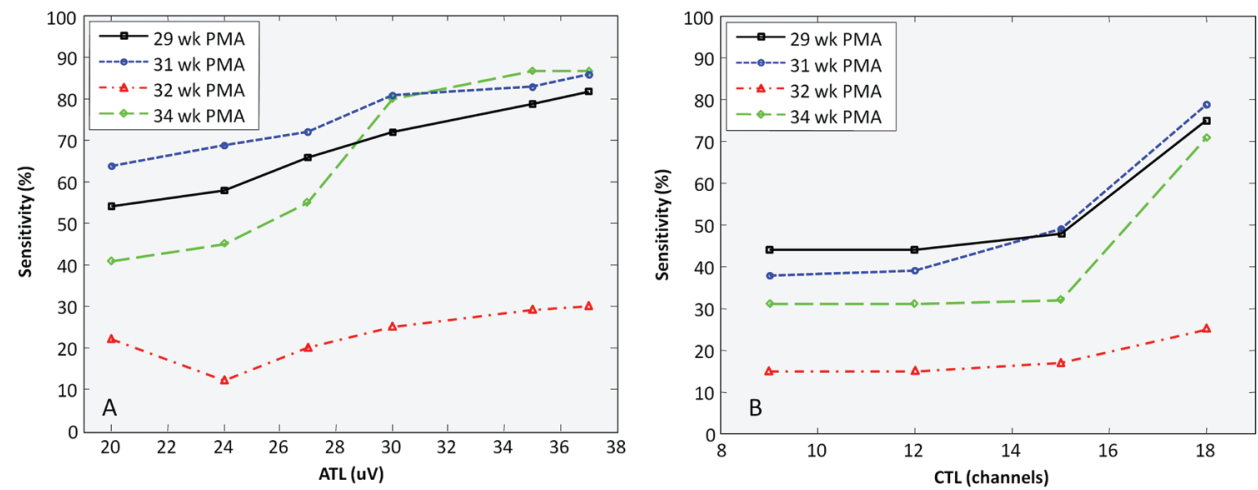

Legend: Resulting sensitivity of IBI classification after applying different threshold values for the amplitude threshold ATL for |BI detection.

consisted of four EEG recordings obtained from four different preterm infants with a comparable PMA as the training set. From each of the EEG recordings, two epochs of $10 \mathrm{~min}$ of data were selected at 30 and 60 min after start of registration. The two selections of an EEG had to contain 
all three classifications. One part had to be mainly discontinuous (representing sleep state) and the other mainly continuous (representing wake state). This was verified by the time percentages of events detected by the algorithm.

\section{Statistics}

Because the training set only consists of the agreed annotations of both observers, remaining parts of EEG are undefined. Therefore, only the number of true positives and false negatives can be determined and the number of false positives and true negatives is unknown. True positives are detections where observer1, observer2 and the algorithm agreed. False negatives are events annotated by both observers but missed by the algorithm. From the annotations, only sensitivity values (the percentage of actual annotations which are correctly identified) can be calculated, which are used for optimizing threshold parameters. Specificity and PPV cannot be determined.

For evaluation of the algorithm, a measure of the operation of the algorithm was obtained by calculation of the sensitivity and PPV for each type of classification, representing the percentage of correct detections. True positive was defined as an event detected by the algorithm and correctly assessed by both observers. A false positive was a detection of the algorithm with which the observers could not agree. For calculation of the sensitivity, false negatives were defined as events missed by the algorithm. These were indicated when the observers did not agree with the detection, when an event was correctly detected but within that detection another event was also present, or when an undefined event had to be classified as a burst, IBI or continuous pattern.

\section{Results}

\section{Training}

The $4 \mathrm{~h}$ training set consisted of 307 bursts, $451 \mathrm{lBls}$ and 35 continuous annotations. The examined threshold values for the amplitude threshold of the high-voltage activity, ATH, were 25 to $60 \mu \mathrm{V}$ in steps of $5 \mu \mathrm{V}$. Because the threshold values for the detection of high-voltage activity are applied in the first part of the algorithm, the value of this parameter influences the detection of burst, IBI as well as continuous pattern. The resulting sensitivity for detection of the three classifications is shown in figure 2 .

From figure 2 it can be seen that the optimal value of ATH for detection of burst and continuous pattern was approximately $30 \mu \mathrm{V}$. For burst and IBI, ATH values higher than $30 \mu \mathrm{V}$ showed a larger sensitivity; however the detection of continuous patterns decreases dramatically for larger amplitude threshold values.

The examined threshold values for the number of channels for high-amplitude activity (CTH) were four, six and eight channels. Two of the EEG-electrodes, i.e. $\mathrm{T}_{3}$ and $\mathrm{T}_{4}$, are present in three EEG-channels. Therefore, the minimum number of channels in which high amplitude activity needs to be present was four channels. The highest value for the number of channels was set at eight channels to prevent missing large parts of high-amplitude activity.

The resulting values of sensitivity of the detections with these threshold values are shown in figure 3. 
Table 1: Optimized threshold values for detection parameters.

\begin{tabular}{|c|c|c|c|c|}
\hline & Amplitude & Time span & Nr. Channels & Nr. Electrodes \\
\hline Burst & $>30 \mu \mathrm{V}$ & $<20 s$ & $\geq 4$ & $\geq 2$ \\
\hline$|\mathrm{IB}|$ & $<30 \mu \mathrm{V}$ & $>1 \mathrm{~s}$ & 18 & 9 \\
\hline Continuous & $>30 \mu \mathrm{V}$ & $\geq 20 \mathrm{~s}$ & $\geq 4$ & $\geq 2$ \\
\hline
\end{tabular}

Table 2: Classifications resulting from algorithm evaluation.

\begin{tabular}{|c|c|c|c|}
\hline PMA & Burst & IBI & Continuous \\
\hline 29 weeks & 67 & 100 & 8 \\
\hline 31 weeks & 53 & 63 & 12 \\
\hline 32 weeks & 71 & 68 & 9 \\
\hline 34 weeks & 42 & 43 & 14 \\
\hline Total & 233 & 274 & 43 \\
\hline
\end{tabular}

Legend: Number of detected burst, IBI and continuous patterns from analysis of the algorithm evaluation EEG records, shown per record/PMA.

Table 3: Evaluation of the burst detection algorithm.

\begin{tabular}{|c|c|c|c|c|c|c|}
\hline & \multicolumn{2}{|c|}{ Burst } & \multicolumn{2}{|c|}{ IBI } & \multicolumn{2}{|c|}{ Continuous } \\
\hline & SNS (\%) & PPV (\%) & SNS (\%) & PPV (\%) & SNS (\%) & PPV (\%) \\
\hline 29 weeks PMA & $86 / 86$ & 93 / 91 & $88 / 85$ & 97 / 97 & $100 / 100$ & $100 / 100$ \\
\hline 31 weeks PMA & $95 / 81$ & $77 / 83$ & 82 / 68 & $100 / 94$ & $100 / 100$ & 83 / 83 \\
\hline 32 weeks PMA & $97 / 85$ & 94 / 85 & 84 / 64 & 94 / 96 & $100 / 83$ & $100 / 60$ \\
\hline 34 weeks PMA & 95 / 97 & $100 / 79$ & $87 / 82$ & $91 / 95$ & 100 / 92 & $71 / 86$ \\
\hline mean \pm sd & $90 \pm 6$ & $88 \pm 8$ & $80 \pm 9$ & $96 \pm 3$ & $97 \pm 5$ & $85 \pm 15$ \\
\hline
\end{tabular}

Legend: Sensitivity (SNS) and positive predictive value (PPV) are shown for both observers using notation (obs1 / obs2).

From figure 3 it can be seen that the optimal value of the number of channels threshold

CTH for burst detection is four EEG-channels. For all three types of classification, the highest sensitivity was achieved at this value.

For determination of threshold values associated with IBI, only sensitivity values of the IBI detection needed to be analyzed. IBI detection is performed in the second part of the algorithm, after the detection of bursts and continuous patterns, and therefore not of influence on the first part of detection. Threshold values for ATL ranged from 20 to $35 \mu \mathrm{V}$. From histogram analysis it appeared that the amplitude of the IBI was higher than $20 \mu \mathrm{V}$ in most of the annotations. Therefore only values higher than $20 \mu \mathrm{V}$ were examined for ATL. Figure 2 shows that the optimal ATH was 
below $35 \mu \mathrm{V}$. Because ATL $<$ ATH, no values larger than $35 \mu \mathrm{V}$ had to be studied for ATL. The resulting values of sensitivity are shown in figure 4 .

The sensitivity of IBI detection increases for larger values of ATL, as can be seen from figure 4 . With the results of ATH optimization and the boundary condition of ATL $\angle A T H, 30 \mu \mathrm{V}$ was determined to be the optimal value for high-voltage activity and IBI detection. ATH was therefore defined as activity equal to and above $30 \mu \mathrm{V}$ while ATL was defined as activity below $30 \mu \mathrm{V}$.

An overview of the threshold values for all the detection parameters resulting from the sensitivity optimization is shown in table 1.

\section{Evaluation}

Evaluation of the algorithm resulted in detection of 234 bursts, 274 IBIs and 44 continuous segments, as indicated in table 2 . The results of the evaluation procedure described in the preceding section are shown in table 3 for each type of classification.

For burst detection, the mean sensitivity value of both observers was $90 \% \pm 6 \%$. Mean sensitivity for observer1 was $93 \% \pm 5 \%$, and mean sensitivity for observer2 was $87 \% \pm 7 \%(p=0.24)$. These values show that observer2 indicated that the algorithm missed bursts more often than observer1. False negatives, bursts missed by the algorithm, mainly consisted of continuous activity (45\%) or undefined events (39\%).

The mean PPV was $88 \% \pm 8 \%$. On average, observer1 agreed with $91 \% \pm 10 \%$ of the detected bursts and observer 2 agreed with $85 \% \pm 5 \%$ of the bursts $(p=0.34)$. For observer1, three records had a PPV above 90\% whilst observer2 had only one. A large difference in PPV for both observers was seen for the infant with a PMA of 34 weeks (100\% and $79 \%$, respectively). A large number of false positives, $46 \%$ on average, were erroneously detected by the algorithm due to the presence of movement or muscle artifacts.

For IBI detection, the mean sensitivity was $80 \% \pm 9 \%$. Similar to burst detection, observer2 indicated that IBIs were missed by the algorithm more often than observer1, which resulted in a lower sensitivity value for all records. Mean sensitivity was $85 \% \pm 3 \%$ for observer 1 and $75 \% \pm 10 \%$ for observer2 $(p=0.08)$. The records of 31 and 32 weeks PMA show the largest difference in sensitivity between both observers. False negatives of IBI detection mostly originated from undefined events, approximately $60 \%$ according to the observers. The number of bursts and continuous patterns that should have been classified as IBI were approximately $20 \%$ each, according to both observers.

The mean PPV value was $96 \% \pm 3 \%$, indicating high agreement with detected IBI. On average, the PPV was $96 \% \pm 4 \%$ for observer1 and $96 \% \pm 1 \%$ for observer2 $(p=1.0)$.

According to the observers, the small amount of incorrectly detected IBI should be classified as burst or undefined event. Observer1 indicated that $20 \%$ of the false positives should have been burst and $80 \%$ undefined. For observer2 this was $50 \%$ and $33 \%$, respectively.

Evaluation of continuous pattern detection shows a mean sensitivity of $97 \% \pm 5 \%$. It should however be noted that the number of evaluated continuous patterns (43) is smaller than the number of bursts and IBIs ( 233 and 274, respectively). Observer 1 had a mean sensitivity value of $100 \% \pm 0 \%$ while the average sensitivity value of observer2 was $94 \% \pm 8 \%(p=0.22)$. Observer2 indicated that a continuous event was missed in the analysis of two records, at 32 and 34 weeks PMA. These two missed continuous patterns originated from one IBI and one undefined event. 
The overall mean PPV was $85 \% \pm 5 \%$. On average, observer1 agreed with $86 \% \pm 14 \%$ and observer2 agreed with $82 \% \pm 17 \%$ of the detections $(p=0.63$ ). For both observers, three PPVs were above $80 \%$. The other records, not the same for both observers, had a PPV of $71 \%$ and $60 \%$, respectively. Both observers agreed that continuous patterns which were detected incorrectly by the algorithm should mainly be subdivided into several bursts and IBIs. A few continuous patterns were detected incorrectly due to the presence of movement- and muscle-artifacts.

Finally, the characteristics of detected events from the evaluation dataset were studied. From

Figure 5: Characteristics of detected events from evaluation datasets.
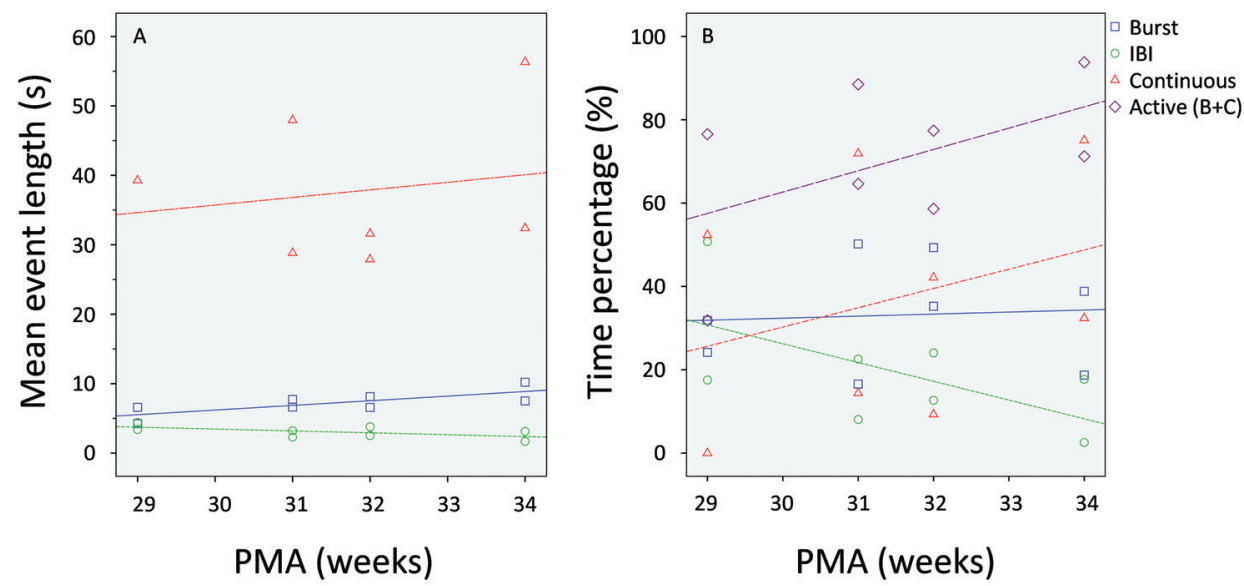

Legend: A) Mean event length for burst ( $\square)$, IBI (O) and continuous activity $(\triangle)$ as detected in the datasets used for evaluation of the algorithm, consisting of two 10-minute EEG segments per PMA. Linear regression of event length as function of PMA, indicated by the regression lines, shows $r=0.76(p=0.03)$ for burst, $r=0.61(p=0.11)$ for IBI and $r=0.18$ ( $p=0.70)$ for continuous activity. B) Time percentage of total recording occupied by detected events for burst, IBI and continuous activity, also showing total activity $(\diamond)$, consisting of combined burst and continuous events. Linear regression of time percentage as function of PMA, indicated by the regression lines, shows $r=0.07(p=0.86)$ for burst, $r=0.60(p=0.12)$ for IBI, $r=0.32$ ( $p=0.45)$ for continuous activity and $\mathrm{r}=0.51(\mathrm{p}=0.20)$ for total activity.

figure 5a, showing mean event length as function of PMA, it can be seen that burst length increases significantly with age ( $p=0.03$ ), while IBI length shows a decreasing trend (not significant: $p=0.11$ ). No change in continuous segment length was observed $(p=0.70)$.

Because the number of detected events (table 2 ) and the mean event length vary with age, the total time percentage occupied by each event also changes as function of PMA, as shown in figure $5 b$. From this figure, it can be seen that the percentage of burst activity in the recordings does not change with PMA, while the IBI percentage shows a decreasing trend $(p=0.12)$. Continuous activity shows low correlation with PMA $(p=0.45)$. However, when observing overall activity (defined as burst plus continuous events), an increasing trend can be seen $(p=0.20)$. The large variation in time percentage values (figure $5 b$ ) results from the selection criterion used for the evaluation segments. 


\section{Discussion}

The aim of our study was to develop an automatic detection method for classification of periods of burst, interburst and continuous activity, serving as a tool for the neurophysiologist to quantify the development of the EEG of healthy preterm infants. An algorithm was developed which classifies the EEG of preterm infants with PMA between 29 and 34 weeks. Detection parameters consisted of amplitude, time span, number of channels and number of electrodes involved with the event. Threshold values for the detection parameters were set based on both literature and training datasets with annotations of two independent observers. The algorithm was evaluated by examining the detections of the algorithm of four EEG recordings, different from the training set, by the same two observers.

There are few studies describing detection of EEG features in preterm infants. Palmu et al. compared visual and automated burst detection in single-channel EEG recordings (11 $\mathrm{min}$ ) of 12 extremely preterm (GA: 23-27 weeks) and 6 very preterm (GA: 28-30 weeks) infants, acquired during first 3 days of life [21]. No information about follow-up results was presented. Automated detection was performed using the nonlinear energy operator. Pfürtscheller et al. detected bursts using a threshold for spontaneous signal variance in single-channel EEG recordings ( $2-3 \mathrm{~h}$ ) obtained from 15 healthy preterm infants (GA:31-35 weeks, PMA:35-37 weeks) [20]. Both these methods are based on detection from single-channel recordings, optimized for longitudinal cerebral monitoring. Varying spatial configuration of EEG transients may favor detection based on multi-channel measurements for in-detail study of maturation [22]. Löfhede et al. studied full term neonates with perinatal asphyxia. Burst detection was performed in eight-channel EEG recordings (6-40 min) using a selection of EEG features optimized with various (nonlinear) classifier methods [23, 29, 30]. Training was performed with one dataset, while evaluation consisted of analyzing six datasets. Due to the different study population, i.e. asphyxia versus normal and full-term versus preterm, direct translation of the results of this study is not possible. Furthermore, straightforward interpretation of classifier results, a nonlinear combination of signal features, can be difficult.

For training of the algorithm, annotations of two independent observers were used.

Overall, annotations made by the observers were comparable. However, when viewing the annotations in detail, e.g. with respect to the onset and offset of events, burst and IBI resulted in a fair agreement $(66 \% \pm 3 \%$ and $70 \% \pm 6 \%$, respectively), while the annotations of continuous patterns had a relatively low agreement $(55 \% \pm 1 \%)$. According to the neurophysiologists, this was mostly due to a different interpretation of tracé alternant, representing either continuous or burst-interburst activity. Although Palmu et al. report high interobserver agreement for burst classification with three observers, $86 \%$ for extremely preterm infants and $81 \%$ for very preterm infants, in general moderate interobserver agreement is often seen for visual EEG interpretation [31, 32]. Therefore, only annotations with agreement of both observers were used to train the algorithm.

In our study, evaluation of the developed algorithm showed that both observers agreed with more than $80 \%$ of all classifications. For burst detection, sensitivity is above $95 \%$ for PMA $>30$ weeks. These results compare well to sensitivity values obtained by Palmu for burst detection in extremely preterm infants. There is sparse data from other publications describing EEG burst detection performance in general, and algorithm sensitivity values in specific. Vairavan performed 
burst detection on MEG data of 12 healthy full-term neonates using Hilbert phase-based detection, which resulted in a low sensitivity of $54 \%$ [33].

For the detection of $|\mathrm{B}|$, the overall mean sensitivity value obtained in our study was also above $80 \%$. Highest sensitivity values were scored for the continuous pattern; both observers had a mean sensitivity value above $94 \%$. Within the values of PPV and sensitivity for each of the events, no trends relating to age were visible. The high mean values of sensitivity and PPV are promising for the usability of the algorithm to detect bursts, IBIs and continuous patterns for cerebral healthy preterm infants.

For burst detection, a large number of false positives, $46 \%$ on average, were incorrectly detected due to the presence of muscle or movement artifacts. Muscle artifacts are often classified as bursts or continuous patterns due to the presence of high-voltage activity. They could be detected based on the relatively high frequency content compared to burst activity. Movement artifacts could be identified by analyzing the simultaneously recorded polysomnographic channels. Incorporating artifact detection and rejection into the developed algorithm could improve PPV.

For IBI detection, analysis of false negatives showed that activity was often falsely classified as undefined ( $58 \%$ of false negatives). Because these false negatives were adjacent to segments of correctly detected IBI, it was concluded that the algorithm only detected partial IBI. There are several possibilities for improvement of IBI detection. Lowering the IBI channel threshold or increasing the IBI amplitude threshold might improve IBI detection, but a more thorough analysis of (more) annotations is recommended. Another option could be to compare undefined segments to adjacent detections, which resembles visual inspection because the detections are then based on interpretation of the continuous signal. After comparison, it can be decided if an undefined event belongs to an IBI.

For detection of continuous patterns, improvement should be focused on reduction of the number of false positives. According to the observers, $90 \%$ of the incorrectly detected continuous patterns should have been subdivided into burst and IBI ( $46 \%$ and $44 \%$ of false positives, respectively). This was most likely caused by the presence of tracé alternant, which resembles a continuous pattern due to relatively high IBI amplitude and low burst amplitude.

In the literature it is described that tracé alternant is visible at the age of 34 weeks PMA [7]; however the pattern changes from tracé discontinu to tracé alternant are gradual and therefore difficult to analyze and quantify. The gradual changes suggest that age-dependent threshold values might improve the algorithm, especially if a larger age range would be studied.

\section{Limitations}

First, the limited range of PMA makes extrapolation of the results to a larger age range difficult.

Studying infants with increasing PMA will provide more information about necessity of age dependent threshold values for the detection parameters and will validate the algorithm for a larger age range.

Second, the number of datasets used for training and evaluation was relatively low; however the number of annotated and detected events within these datasets was high. The training set consisted of 307 bursts, 451 IBIs and 35 continuous segments. Evaluation of the algorithm resulted in detection of 234 bursts, $274 \mathrm{IBI}$ and 44 continuous segments. These numbers of datasets and 
bursts for training and evaluation of our algorithm are comparable to the numbers used in burst detection studies mentioned previously $[21,23,34]$. Annotations of more children of the same GA and annotations of more observers could increase detection robustness.

Third, in this study the asynchrony of bursts, age-dependent transients and sleep-wake cycles were not taken into account. Burst asynchrony could influence detection sensitivity due to the applied channel threshold, possibly resulting in under-detection. In the literature it was found that $80-100 \%$ of bursts are synchronous for the preterm younger than 30 weeks PMA [7]. After 30 weeks PMA burst synchrony decreases to $50-90 \%$ where after it increases again to $100 \%$ at 40 weeks PMA. According to the observers, asynchronous bursts were not present in the datasets included in this study. We focused on the detection of burst, IBI and continuous activity because these relate to normal maturation as well as being possible indicators of pathology, e.g. perinatal asphyxia [35, 36].

Maturation of cerebral activity is characterized by continuity and age-dependent transients, e.g. delta brush, theta wave, temporal saw tooth. For identification of these transients within detected EEG bursts, a shape analysis algorithm could be applied [37]. We did not address sleep behavior of preterm infants; therefore between-state differences were not analyzed. To maximize the robustness of the method, we refrained from performing analysis on sub-segments, in order to reduce bias based on user information and to avoid possible errors in sample selection.

Fourth, because the exact onset and offset of events were not taken into account with the evaluation of the algorithm, absolute quantification of burst, interburst and continuous segment length from detected events remains a confining factor. Because detections are based on objective thresholds, the algorithm delivers reproducible results. Therefore, application of our algorithm in studies of maturation, e.g. focused on relative continuity changes with age, is feasible. Analysis of event detections from the evaluation datasets shows that the percentage of discontinuous activity (burst + IBI) decreases from approximately $63 \%$ at 29 weeks to $43 \%$ at 34 weeks. These findings correspond to studies by Herbertz et al., showing that for the infant younger than 30 weeks PMA most recordings will consist of only discontinuous EEG [38]. The percentage of discontinuous activity decreases with increasing PMA from about $64 \%$ at $27-28$ weeks to approximately $45 \%$ at 31-32 weeks and is no longer present in any state after 46 weeks PMA.

In conclusion, a simple algorithm was developed detecting bursts, IBIs and continuous patterns in the EEG of preterm infants. Evaluation showed high sensitivity and PPVs for the algorithm. Computer-assisted analysis of EEG may allow an objective and reproducible analysis for prognosis and stratification of clinical treatment. 
1. Anderson P, Doyle LW. Neurobehavioral outcomes of school-age children born extremely low birth weight or very preterm in the 1990s. Jama 2003;289(24):3264-72.

2. Bhutta AT, Cleves MA, Casey PH, Cradock MM, Anand KJ. Cognitive and behavioral outcomes of school-aged children who were born preterm: a meta-analysis. Jama 2002;288(6):728-37.

3. Volpe JJ. Brain injury in premature infants: a complex amalgam of destructive and developmental disturbances. Lancet Neurol 2009;8(1):110-24.

4. Biagioni E, Bartalena L, Boldrini A, Cioni G, Giancola S, Ipata AE. Background EEG activity in preterm infants: correlation of outcome with selected maturational features. Electroencephalogr Clin Neurophysiol 1994;91(3):154-62.

5. Holmes GL, Lombroso CT. Prognostic value of background patterns in the neonatal EEG. J Clin Neurophysiol 1993;10(3):323-52.

6. KundelHL, PolanskyM. Measurement of observer agreement. Radiology 2003;228(2):303-8.

7. Lombroso CT. Neonatal polygraphy in full-term and premature infants: a review of normal and abnormal findings. J Clin Neurophysiol 1985;2(2):105-55.

8. Tharp BR, Cukier F, Monod N. The prognostic value of the electroencephalogram in premature infants. Electroencephalogr Clin Neurophysiol 1981;51(3):219-36.

9. Hayakawa M, Okumura A, Hayakawa F, Watanabe K, Ohshiro M, Kato Y, et al. Background electroencephalographic (EEG) activities of very preterm infants born at less than 27 weeks gestation: a study on the degree of continuity. Arch Dis Child Fetal Neonatal Ed 2001;84(3):F163-7.

10. Scher MS. Normal electrographic-polysomnographic patterns in preterm and fullterm infants. Semin Pediatr Neurol 1996;3(1):2-12.

11. Stockard-Pope JE, Werner SS, Bickford R. Ontogenesis of EEG in Premature Infants. In: Stockard-Pope JE, Werner SS, Bickford R, editors. Atlas of Neonatal Electroencephalography. New York: Raven Press; 2006. p. 105-175.

12. Binnie CD, Cooper R, Maguiere F, Osselton JW, Prior PF, Tedman B. Neurophysiology of the neonatal period. In: Clinical Neurophysiology, Volume 2, EEG, Pediatric Neurophysiology, Special Techniques and Applications. Amsterdam: Elsevier; 2003.

13. Vecchierini MF, Andre M, d'Allest AM. Normal EEG of premature infants born between 24 and 30 weeks gestational age: terminology, definitions and maturation aspects. Neurophysiol Clin 2007;37(5):311-23.

14. Victor S, Appleton RE, Beirne M, Marson AG, Weindling AM. Spectral analysis of electroencephalography in premature newborn infants: normal ranges. Pediatr Res 2005;57(3):336-41.

15. PaulK, Krajca V, Roth Z, Melichar J, Petranek S Comparison of quantitative EEG characteristics of quiet and active sleep in newborns. Sleep Med 2003;4(6):543-52.

16. Selton D, Andre M, Hascoet JM. Normal EEG in very premature infants: reference criteria. Clin Neurophysiol 2000;111(12):2116-24.

17. Vecchierini MF, d'Allest AM, Verpillat P. EEG patterns in 10 extreme premature neonates with normal neurological outcome: qualitative and quantitative data. Brain Dev 2003;25(5):330-7.

18. Hellstrom-Westas L, Klette H, ThorngrenJerneck K, Rosen I. Early prediction of out come with aEEG in preterm infants with large intraventricular hemorrhages. Neuropediatrics 2001;32(6):319-24.

19. Wikstrom S, Ley D, Hansen-Pupp I, Rosen I, Hellstrom-Westas L. Early amplitude-integrated EEG correlates with cord TNF-alpha and brain 
injury in very preterm infants. Acta Paediatr 2008;97(7):915-9.

20. Pfurtscheller K, Bauernfeind G, Muller-Putz GR, Urlesberger B, Muller W, Pfurtscheller G. Correlation between EEG burst-to-burst intervals and HR acceleration in preterm infants. Neurosci Lett 2008;437(2):103-6.

21. Palmu K, Wikstrom S, Hippelainen E, Boylan G, Hellstrom-Westas L, Vanhatalo S. Detection of 'EEG bursts' in the early preterm EEG: visual vs. automated detection. Clin Neurophysiol 2010;121(7):1015-22.

22. Vanhatalo S, Kaila K. Development of neonatal EEG activity: from phenomenology to physiology. Semin Fetal Neonatal Med 2006;11(6):471-8.

23. Lofhede J, Degerman J, Lofgren N, Thordstein $M$, Flisberg A, Kjellmer l, et al. Comparing a supervised and an unsupervised classification method for burst detection in neonatal EEG. Conf Proc IEEE Eng Med Biol Soc 2008;2008:3836-9.

24. Niemarkt HJ, Andriessen P, Peters CH, Pasman JW, Blanco CE, Zimmermann LJ, et al. Quantitative analysis of amplitude-integrated electroencephalogram patterns in stable preterm infants, with normal neurological development at one year. Neonatology 2010;97(2):175-82.

25. Niemarkt HJ, Andriessen P, Peters $\mathrm{CH}$, Pasman JW, Zimmermann LJ, Bambang Oetomo S. Quantitative analysis of maturational changes in EEG background activity in very preterm infants with a normal neurodevelopment at 1 year of age. Early Hum Dev 2010;86(4):219-24.

26. Bayley N. Manual for Bayley Scales of Infant Development. 2nd Edition. San Antonio, Texas: The Psychological Corporation; 1993.

27. Tekgul H, Bourgeois BF, Gauvreau K, Bergin AM. Electroencephalography in neonatal seizures: comparison of a reduced and a full 10/20 montage. Pediatr Neurol 2005;32(3):155-61.

28. Conde JR, de Hoyos AL, Martinez ED, Campo
CG, Perez AM, Borges AA. Extrauterine life duration and ontogenic EEG parameters in preterm newborns with and without major ultrasound brain lesions. Clin Neurophysiol 2005;116(12):2796-809.

29. Lofhede J, Lofgren N, Thordstein M, Flisberg A, Kjellmer I, Lindecrantz K. Classification of burst and suppression in the neonatal electroencephalogram. J Neural Eng 2008;5(4):402-10.

30. Lofhede J, Lofgren N, Thordstein M, Flisberg A, Kjellmer I, Lindecrantz K. Comparison of three methods for classifying burst and suppression in the EEG of post asphyctic newborns. Conf Proc IEEE Eng Med Biol Soc 2007;2007:5136-9.

31. Abend NS, Gutierrez-Colina A, Zhao H, Guo $R$, Marsh E, Clancy RR, et al. Interobserver reproducibility of electroencephalogram interpretation in critically ill children. J Clin Neurophysiol;28(1):15-9.

32. Gerber PA, Chapman KE, Chung SS, Drees C, Maganti RK, Ng YT, et al. Interobserver agreement in the interpretation of EEG patterns in critically ill adults. J Clin Neurophysiol 2008;25(5):241-9.

33. Vairavan S, Eswaran H, Haddad N, Rose DF, PreissI H, Wilson JD, et al. Detection of discontinuous patterns in spontaneous brain activity of neonates and fetuses. IEEE Trans Biomed Eng 2009;56(11 Pt 2):2725-9.

34. Pfurtscheller K, Muller-Putz GR, Urlesberger B, Dax J, Muller W, Pfurtscheller G. Synchronous occurrence of EEG bursts and heart rate acceleration in preterm infants. Brain Dev 2005;27(8):558-63.

35. Holmes G, Rowe J, Hafford J, Schmidt R, Testa $M$, Zimmerman A. Prognostic value of the electroencephalogram in neonatal asphyxia. Electroencephalogr Clin Neurophysiol 1982;53(1):60-72.

36. Sinclair DB, Campbell M, Byrne P, Prasertsom W, Robertson CM. EEG and long-term outcome 
of term infants with neonatal hypoxicischemic encephalopathy. Clin Neurophysiol 1999;110(4):655-9.

37. Mitchell TJ, Inder TE, Neil JJ. Automated interpretation of premature EEG using Bayesian methods. In: Pediatric Academic Society/ASPR 2011; 2011; Denver; 2011.

38. Herbertz S, Pulzer F, Gebauer C, Panhofer M, Robel-Tillig E, Knupfer M. The effect of maturation and sedation on amplitude-integrated electroencephalogram of the preterm neonate: results of a prospective study. Acta Paediatr 2006;95(11):1394-9. 



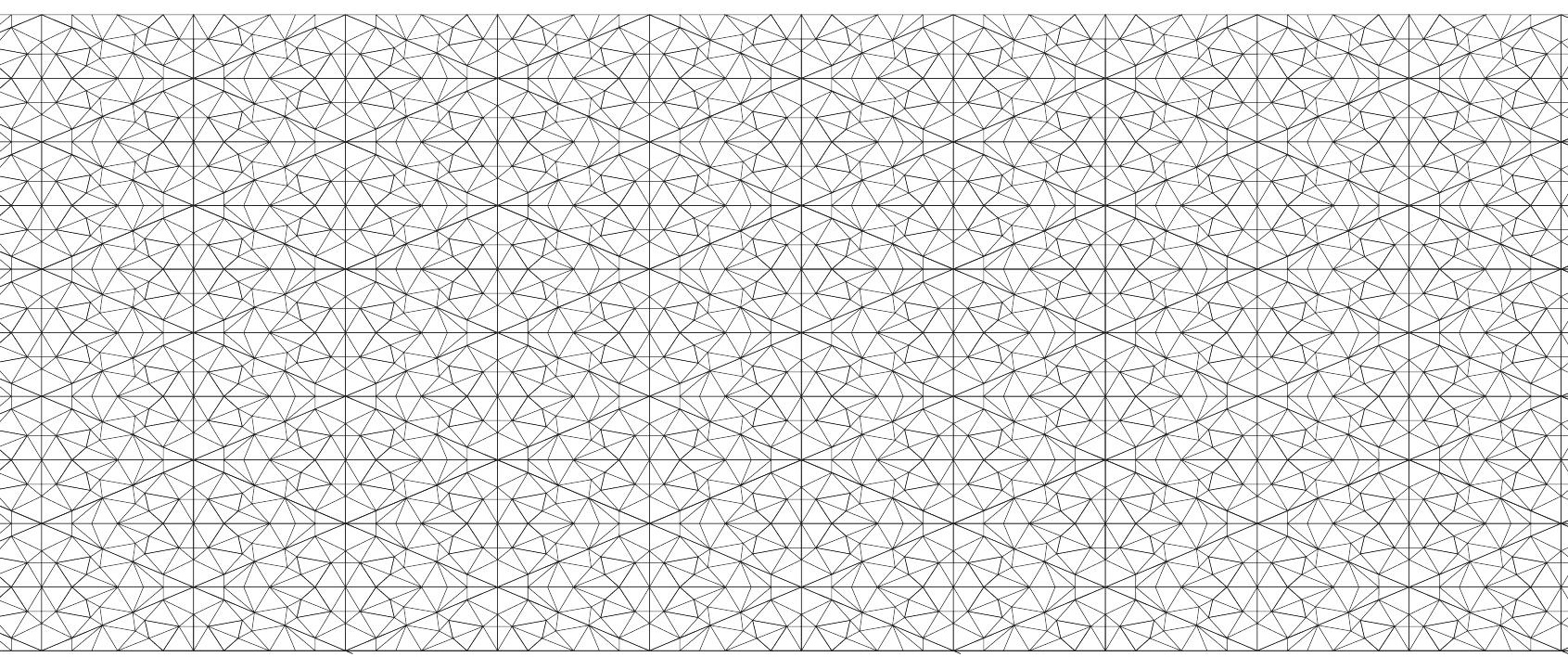




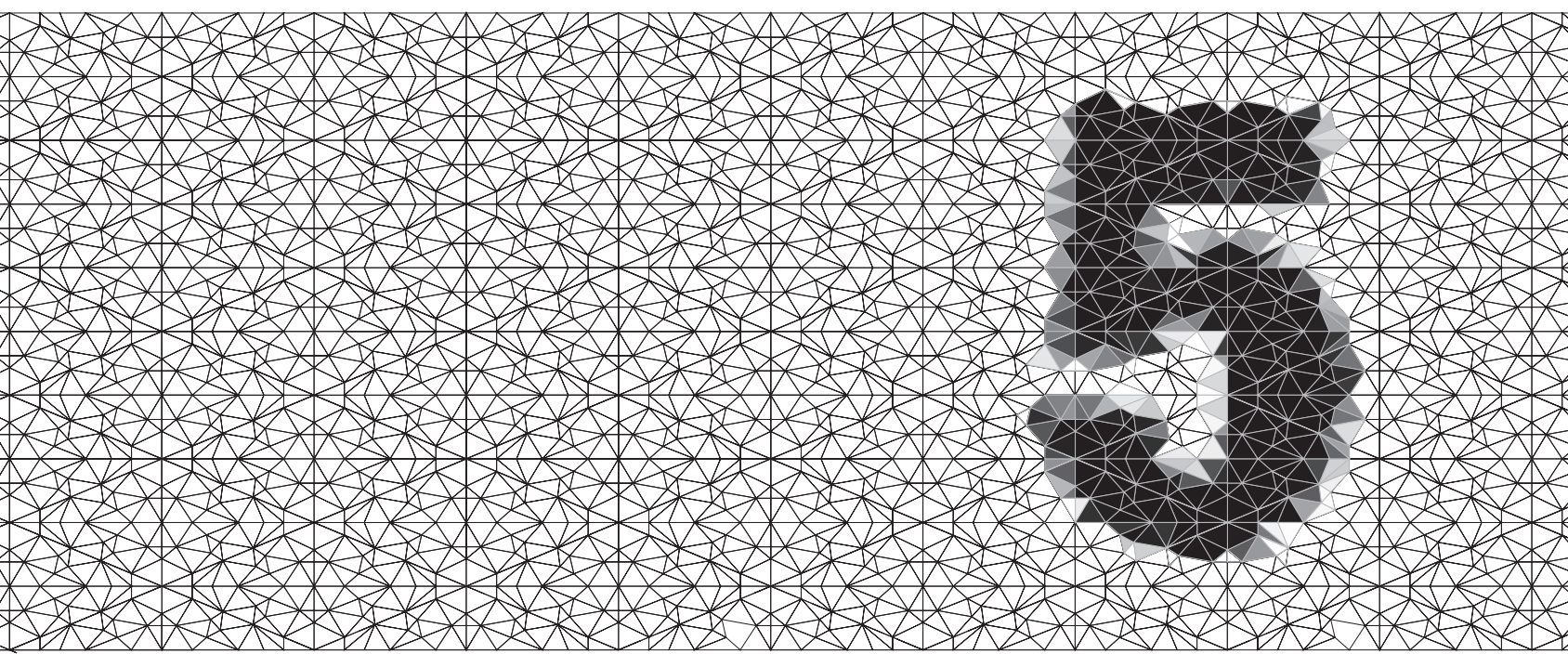

\title{
Maturational Changes in Automated EEG Spectral Power Analysis in Preterm Infants
}

\author{
Hendrik J. Niemarkt \\ Ward Jennekens \\ Jaco W. Pasman \\ Titia Katgert \\ Carola Van Pul \\ Antonio W.D. Gavilanes \\ Boris W. Kramer \\ Luc J. Zimmermann \\ Sidarto Bambang Oetomo \\ Peter Andriessen
}

Pediatr Res. 2011 Nov;70(5):529-534. 


\section{Abstract}

Our study aimed at automated power spectral analysis of the EEG in preterm infants to identify changes of spectral measures with maturation. Weekly (10-20 montage) 4-h EEG recordings

were performed in 18 preterm infants with $G A<32$ wk and normal neurological follow-up at $2 \mathrm{y}$, resulting in 79 recordings studied from $27+4$ to $36+3 \mathrm{wk}$ of postmenstrual age (PMA, GA + postnatal age).

Automated spectral analysis was performed on 4-h EEG recordings. The frequency spectrum was divided in delta $1(0.5-1 \mathrm{~Hz})$, delta $2(1-4 \mathrm{~Hz})$, theta $(4-8 \mathrm{~Hz})$, alpha $(8-13 \mathrm{~Hz})$, and beta $(13-30$ $\mathrm{Hz}$ ) band. Absolute and relative power of each frequency band and spectral edge frequency were calculated. Maturational changes in spectral measures were observed most clearly in the centrotemporal channels. With advancing PMA, absolute powers of delta 1 to 2 and theta decreased. With advancing PMA, relative power of delta 1 decreased and relative powers of alpha and beta increased, respectively.

In conclusion, with maturation, spectral analysis of the EEG showed a significant shift from the lower to the higher frequencies. Computer analysis of EEG will allow an objective and reproducible analysis for long-term prognosis and/or stratification of clinical treatment. 


\section{Introduction}

Advances in the care of very preterm infants have led to an increased survival [1]. However, a considerable number of these infants experience neurological deficits later in life, even in the absence of neuroimaging abnormalities [2, 3]. The exact etiology of these developmental deficits remains to be clarified, but it is suggested that medical, environmental, and iatrogenic conditions may interfere with white matter development of the vulnerable preterm brain [4]. Therefore, brain function monitoring in preterm infants during their stay in the NICU may be valuable in detecting conditions that interfere with brain development [5]. It is a challenge to develop effective monitoring and therapeutic strategies to protect the preterm brain.

The EEG is regarded as the gold standard in the assessment of cerebral function. Assessing changes in EEG are useful in the prediction of long-term outcome [6]. Although the acute and chronic EEG changes are mainly nonspecific regarding type of damage, they correlate with later neurological and cognitive function [7]. In preterm infants developing white matter damage, acute EEG findings include decreased continuity, lower amplitude of background activity, and epileptic seizure activity [8]. The chronic EEG changes associated with white matter injury and abnormal neurological development include delayed maturation and disorganized pattern with the presence of abundant positive Rolandic sharp waves [9, 10]. In addition, EEG patterns of preterm infants change with postmenstrual age (PMA) [11, 12]. In the very preterm infant, the EEG background activity is characterized by discontinuity, instability, and fragmentation [13]. The greater the prematurity, the more marked are these EEG aspects. These characteristics make the interpretation of the tracings very complex.

As EEG has reproducible patterns in normal very preterm infants, this constitutes a basis for a quantitative analysis of EEG for future neurological prognosis in very preterm infants [14]. Today, digital EEG recorders provide the opportunity to analyze the EEG quantitatively. Scher et al. [15] calculated quantitatively several specific EEG sleep measures with the objective to combine these measures into a single "dysmaturity index," e.g. which best expresses an infant's EEG sleep behavior relative to the average full-term infant. Among other sleep EEG measures, spectral power analysis of the EEG has the potential to discriminate sleep state between preterm and term infants. By using automated spectral analysis, Victor et al. [16] studied 75-min EEG recordings performed in 53 preterm infants $<30 \mathrm{wk}$ of gestation (no follow-up available) during the first $4 \mathrm{~d}$ of life. West et al. [17] analyzed changes in continuity, amplitude, and spectral edge frequency (SEF) with an automated algorithm in 60-min EEG recordings, performed daily in 63 preterm infants < 32 wk of gestation (no follow-up available) during the first week of life. These studies suggest that spectral power analysis of the EEG is useful for brain monitoring and show consistent changes in several quantitative EEG measures over time in preterm infants, indicating functional cerebral maturation. Among limitations of the last two studies are that relatively short recordings were performed during the first days of life with a small range in PMA and no data on long-term follow-up available. As the recordings of these studies were performed during the first week of life, the results may merely reflect the perinatal transition phase rather than postnatal maturation.

The aim of this study was to investigate if neurophysiologic maturation in very preterm infants can be described by automated spectral power analysis of whole 4-h EEG recordings and to identify 
specific changes of spectral power measures with maturation. We performed serial (weekly) 4-h EEG recordings in healthy preterm infants with a $\mathrm{GA}<32 \mathrm{wk}$ and a normal neurodevelopmental follow-up at 2 y of corrected age.

\section{Methods}

\section{Study subjects}

This study was part of a comprehensive EEG research program of Máxima Medical Centre in Veldhoven, The Netherlands, and conducted in the NICU from May 2006 to July 2007. The hospital's ethics committee approved the study. Infants were enrolled after written informed consent from both parents. For details of the study group, we refer to earlier articles $[18,19]$.

During the study period, 95 preterm infants were eligible for the study, on the basis of "firstday" inclusion criteria: preterm infants with a GA $<32$ wk, birth weight appropriate for GA, Apgar score $\geq 6$ at $5 \mathrm{~min}$, arterial umbilical $\mathrm{pH}>7.00$, normal cerebral ultrasonography $<48 \mathrm{~h}$ after birth, and infants who were expected to stay longer than 4 wk in our hospital. Of the eligible population, 67 subjects were subsequently excluded at the end of the first week because of cardiovascular instability (requiring volume expansion and/or inotropic drugs), abnormalities on cranial ultrasound (intraventricular hemorrhage II-IV and all grades of periventricular leucomalacia) [20], and use of any sedative or antiepileptic medication, subjects with recurrent apnea treated with doxapram or no parental consent.

Thus, 28 infants were enrolled in the study. Of these, 10 subjects were excluded from analysis because they met exclusion criteria after the first week $(n=8)$ or showed delayed development at corrected age of $24 \mathrm{mo}(n=2)$. Finally, 18 clinically stable preterm infants with at least 4 weekly EEG recordings and a normal neurological follow-up at corrected age of $24 \mathrm{mo}$ (Bayley Scales of Infant Developmental II for mental and motor function) were analyzed in this article. The characteristics of these subjects are shown in table 1 .

\section{Data acquisition}

Starting at the end of the first week of life, weekly digital EEG recordings (NicoletOne; Viasys Healthcare, Conshohocken, PA) were performed. After skin preparation (Nuprep Gel, D.O. Weaver, Aurora, $\mathrm{CO}$ ), $\mathrm{Ag} / \mathrm{AgCl}$ cup electrodes, filled with a conductive paste (Ten20, D.O. Weaver), were placed according to the international 10 to 20 reduced montage system (figure 1) [21]. The digital EEG signal was sampled at $256 \mathrm{~Hz}$ and stored on a hard disk. Artifacts related to repositioning the infant or replacement of an electrode were removed when impedance value was $<10 \mathrm{k}$ [Omega] or total spectral power value was above empirical threshold value of $10 \times 10^{3} \mu \mathrm{V}^{2}(<5 \%$ of data). All recordings took place from 0800 to $1200 \mathrm{~h}$. During the 4 -h recording, the infants were in prone or side position. Feeding and care for the infant was performed according to the normal routine of the NICU. 
Table 1: Characteristics of study population $(\mathrm{n}=18)$.

\section{Clinical characteristics}

\begin{tabular}{ll}
\hline Gestational age (wk) & $29.0 \pm 0.3$ \\
\hline Birth weight (g) & $1297 \pm 58$ \\
\hline Female rate (\%) & 39 \\
\hline 5-min Apgar score & $8.5 \pm 1.3$ \\
\hline Umbilical arterial pH & $7.28 \pm 0.09$ \\
\hline Tocolysis (\%) & 83 \\
\hline Antenatal corticoids (\%) & 94 \\
\hline Caesarean section (\%) & 28 \\
\hline lowest pH, first week & $7.26+0.04$ \\
\hline lowest glucose (mmol/l), first week & $3.0 \pm 1.1$ \\
\hline MDI at 24-mo & $106 \pm 12$ \\
\hline PDI at 24-mo & $91 \pm 11$ \\
\hline Legend: values expressed as mean \pm SD, percentage or median (range). During the first week of life, the lowest pH and serum \\
glucose value of each subject was noted and expressed as mean \pm SD. Mental Developmental Index (MDI); Psychomotor \\
\hline Developmental Index (PDI).
\end{tabular}

Figure 1: The international 10-20 reduced EEG montage system for neonates.

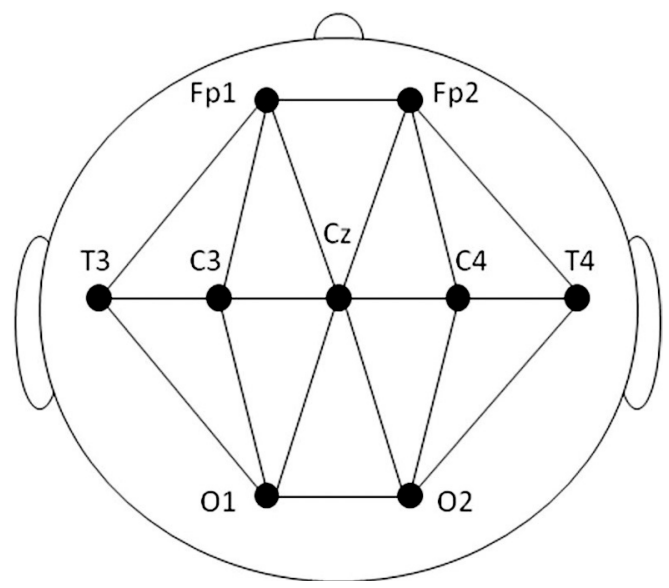

Legend: From the EEG montage the following channels were derived: frontal channel $\mathrm{Fp}_{1}-\mathrm{Fp}_{2}$; central channel $\mathrm{C}_{3}$ - $\mathrm{C}_{4}$; occipital channel $\mathrm{O}_{1}-\mathrm{O}_{2}$; left centro-temporal $\mathrm{C}_{3}-\mathrm{T}_{3}$; right centro-temporal $\mathrm{C}_{4}-\mathrm{T}_{4}$.

\section{Data analysis}

Spectral analysis was performed using Nicolette EEG analyzer software. Data were filtered using 
Figure 2: Regression plots for total spectral power as function of PMA for the different channels.
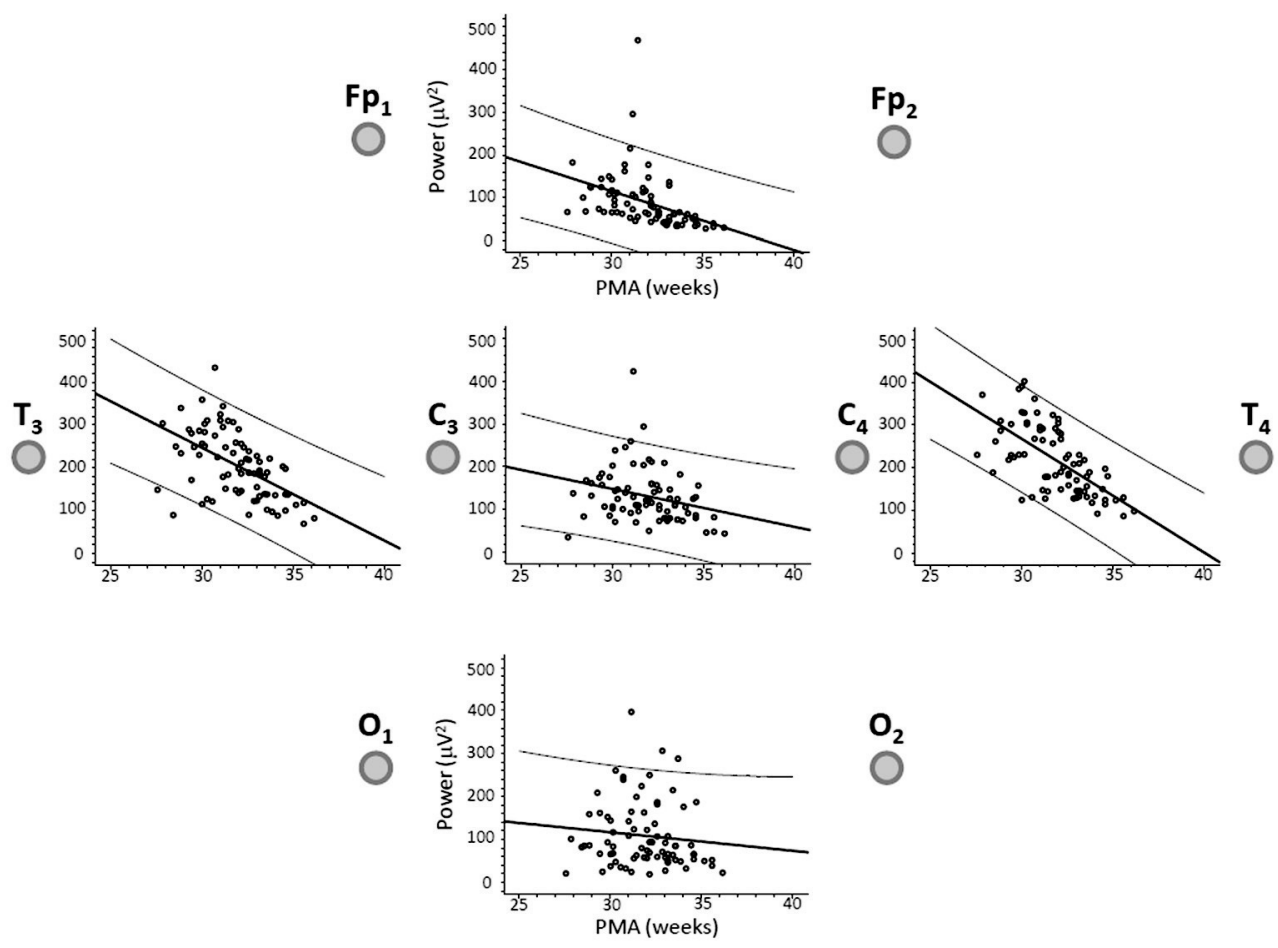

Legend: The relationship between PMA ( $\mathrm{x}$-axis, weeks) and total spectral power ( $\mathrm{y}$-axis, $\mu \mathrm{V}^{2}$ ) is shown for 5 channels. The electrode positions are indicated by dots and accessory channel identification (frontal channel $\mathrm{Fp}_{1}-\mathrm{Fp}_{2}$; central channel $\mathrm{C}_{3}-\mathrm{C}_{4}$; occipital channel $\mathrm{O}_{1}-\mathrm{O}_{2}$ and the left and right centrotemporal channels $\mathrm{C}_{3}-\mathrm{T}_{3}$ and $\mathrm{C}_{4}-\mathrm{T}_{4}$ ). Linear regression lines are shown with 95\% confidence intervals. Except for the occipital channel, the other channels showed significant slopes of the regression lines (b-coefficient $\pm S D)$ : $F p_{1}-F_{2^{\prime}}-13 \pm 4(p<0.01) ; C_{3}-C_{4^{\prime}}-9 \pm 4(p<0.05) ; C_{3}-T_{3^{\prime}}-21 \pm 4(p<0.01) ; C_{4}-T_{4^{\prime}}-25 \pm 4 \mu V^{2} / w k(p<0.01)$.

a $0.5 \mathrm{~Hz}$ high-pass filter and $30 \mathrm{~Hz}$ low-pass filter. Subsequently, data were divided into 4-s half overlapping segments. Each segment was multiplied by a Hamming window before fast

Fourier transform. The frequency spectrum and frequency band powers were determined for each segment and averaged over four consecutive segments, yielding a 10-s time resolution. Finally, the frequency information was averaged over the complete 4-h recording, including periods of continuity and discontinuity, to obtain mean EEG frequency parameters per EEG recording.

Five channels were used for analysis (figure 1): frontal channel, Fp1-Fp2; central channel, $\mathrm{C}_{3}-\mathrm{C}_{4}$; occipital channel, $\mathrm{O}_{1}-\mathrm{O}_{2}$, and the left and right centrotemporal channels, $\mathrm{C}_{3}-\mathrm{T}_{3}$ and $\mathrm{C}_{4}-\mathrm{T}_{4}$. The frequency spectrum was divided into the following bands: delta $1(0.5-1 \mathrm{~Hz})$; delta $2(1-4 \mathrm{~Hz})$; theta (4-8 Hz); alpha (8-13 Hz); and beta $(13-30 \mathrm{~Hz})$.

Three aspects of the transformed signal were calculated automatically: 1 ) absolute power (defined as the integral of all powers within the frequency band, expressed in $\mu V^{2}$ ), 2) relative power (defined as the ratio of absolute band power to total power of all bands, expressed in percentage); and 3) SEF (defined as the frequency that delimits $95 \%$ of the power between 0.5 and $30 \mathrm{~Hz}$ ). 


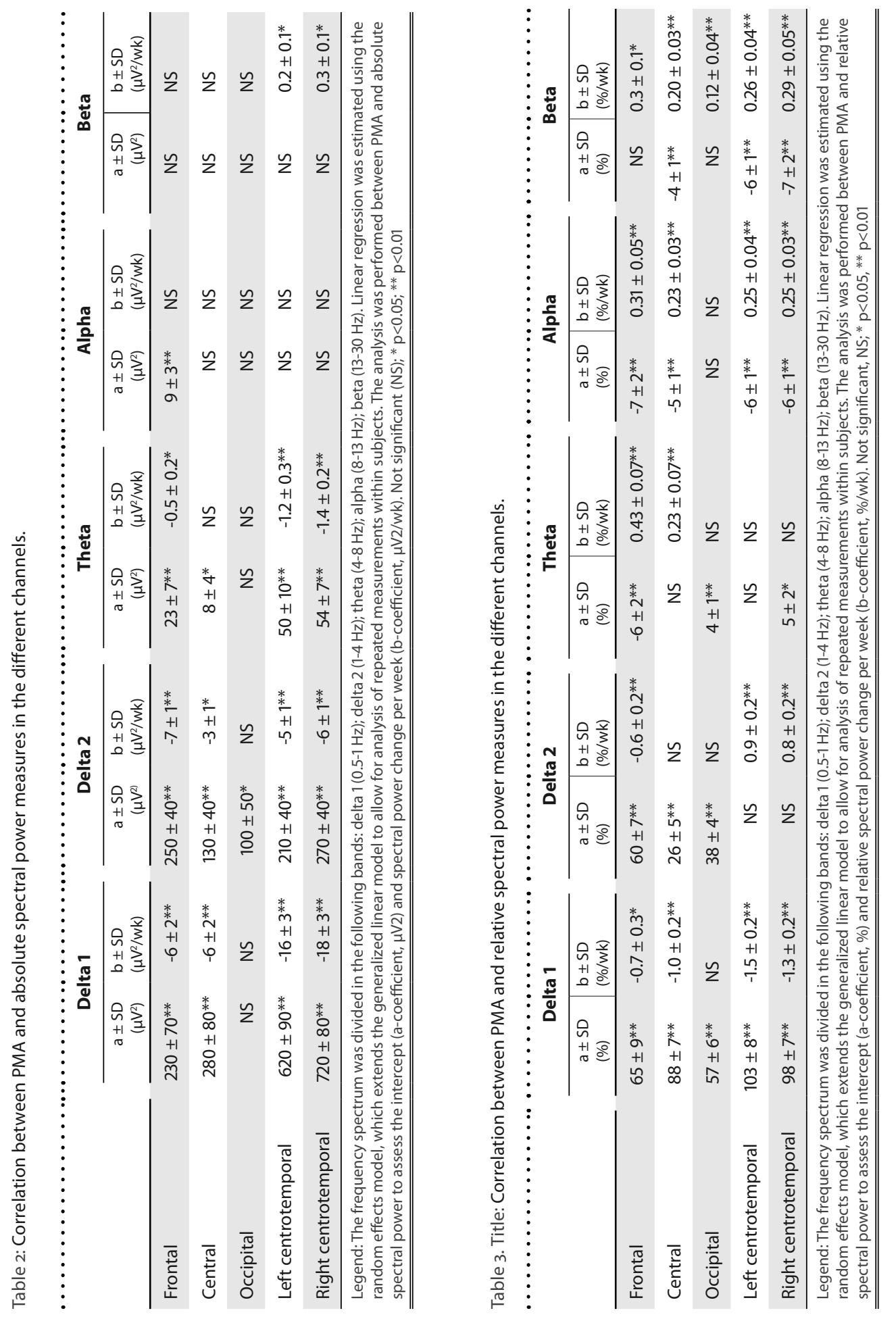


Table 4: Correlation between PMA and spectral edge frequency in the different channels.

\begin{tabular}{|c|c|c|}
\hline & \multicolumn{2}{|c|}{ Spectral Edge Frequency } \\
\hline & $\mathrm{a} \pm \mathrm{SD}(\mathrm{Hz})$ & $\mathrm{b} \pm \mathrm{SD}(\mathrm{Hz} / \mathrm{wk})$ \\
\hline Frontal & NS & $0.4 \pm 0.1^{* *}$ \\
\hline Central & $-7 \pm 2^{* *}$ & $0.37 \pm 0.07^{* *}$ \\
\hline Occipital & NS & NS \\
\hline Left centrotemporal & $-7 \pm 2^{* *}$ & $0.37 \pm 0.07^{* *}$ \\
\hline Right centrotemporal & $-7 \pm 2^{* *}$ & $0.36 \pm 0.07^{* *}$ \\
\hline \multicolumn{3}{|c|}{$\begin{array}{l}\text { Linear regression was estimated using the random effects model, which extends the generalized linear model to allow for } \\
\text { analysis of repeated measurements within subjects. Analysis was performed to assess the intercept (a-coefficient, } \mathrm{Hz} \text { ) and } \\
\text { frequency change per week (b-coefficient, } \mathrm{Hz} / \mathrm{wk} \text { ). Not significant, NS; }{ }^{* *} \mathrm{p}<0.01\end{array}$} \\
\hline
\end{tabular}

\section{Statistical analysis}

Data with a normal distribution were expressed as mean $\pm S D$, otherwise data were expressed as median and interquartile range (IQR). Data were statistically analyzed with SPSS 13.1 (SPSS Inc, Chicago,IL). The relations between PMA and EEG spectral measures were evaluated using multilevel analysis. Linear regression was estimated using the random effects model, which extends the generalized linear model to allow for analysis of repeated measurements between and within subjects. Regression analysis results are shown with the $a$ - and b-coefficient $(a$, intercept; $b$, slope of regression line). A $p$ value $<0.05$ was considered statistically significant.

\section{Results}

Eighteen clinically stable infants (GA, $29.0 \pm 0.3 \mathrm{wk}$; birth weight, $1297 \pm 58 \mathrm{~g}$ ) were studied weekly and underwent four to six EEG recordings (four recordings in $n=13$ infants; five recordings in $n=3$ infants; and six recordings in $n=2$ infants). All 79 EEG recordings (representing a large variability in PMA, $27+4$ to $36+3$ weeks) were pooled into one dataset and analyzed with the generalized linear model. All EEG recordings showed the background patterns generally found in preterm infants: tracé continue (continuous EEG activity of similar voltage and frequency) and tracé discontinue (alternating segments of activity and quiescence). None of the EEG recordings showed evidence of neonatal seizures.

\section{Absolute power}

Figure 2 illustrates the changes of total spectral power with PMA for the different channels. In the frontal (Fp1-Fp2) channel, absolute spectral power measures in the delta and theta band decreased with PMA (table 2). In the central channel ( $\left.C_{3}-C_{4}\right)$, absolute delta spectral powers decreased, while theta, alpha, and beta powers remained unchanged. In the occipital channel, no changes in absolute spectral power measures were observed. In both centrotemporal channels ( $C_{3}-T_{3}$ and $\left.\mathrm{C}_{4}-\mathrm{T}_{4}\right)$, absolute delta and theta spectral powers decreased, while beta power increased. The 
greatest spectral power change per week was observed for the delta 1 spectral power measure in the centrotemporal channels. In general, absolute spectral power in the lower frequency range $(<8 \mathrm{~Hz})$ decreased with PMA.

\section{Relative power}

In the frontal channel, relative delta power decreased, while theta, alpha, and beta powers increased with PMA (table 3). In the central channel, relative delta 1 power decreased, while theta, alpha, and beta relative spectral power measures increased with PMA, respectively. Except for a slight increase in relative beta power, other relative spectral powers remained unchanged in the occipital channel. In both centrotemporal channels, relative delta 1 power decreased, while delta 2, alpha, and beta increased with PMA. In general, relative spectral power measures showed a shift toward the higher frequency ranges with increasing PMA.

\section{Spectral edge frequency}

SEF increased with PMA in all channels, with the exception of the occipital channel (table 4).

\section{Discussion}

We investigated spectral power changes with an automated algorithm based on fast Fourier transform in serial whole 4-h EEG recordings performed during the first 4 to $6 \mathrm{wk}$ after birth. The preterm infants were clinically stable at time of the recording, and assessment at 24 mo showed a normal neurodevelopmental outcome. The main findings are that in normal preterm infants with increasing PMA, absolute spectral power in the lower frequency range decreases and relative spectral power measures show a shift toward the higher frequency ranges and SEF increases.

In general, our observation of decrease in absolute spectral power in the delta $1(0.5-1 \mathrm{~Hz})$ and delta $2(1-4 \mathrm{~Hz})$ band is consistent with others. Okumura et al. [22] demonstrated in 10 clinical stable preterm infants (29-34 wk of gestation, normal follow-up at $18 \mathrm{mo}$ ) a decrease of delta 1 spectral power in all channels with advancing age. Bell et al. [23] studied 20 healthy preterm infants (26-32 wk of gestation, normal follow- up at $18 \mathrm{mo}$ ) and demonstrated a decrease of absolute delta 1 and 2 spectral power with advancing age in the frontal $\left(\mathrm{F}_{3}-\mathrm{C}_{3}\right.$ and $\left.\mathrm{F}_{4}-\mathrm{C}_{4}\right)$ and parietal $\left(\mathrm{C}_{3}-\mathrm{P}_{3}\right.$ and $\left.\mathrm{C}_{4}-\mathrm{P}_{4}\right)$ channels. Scher et al. [24] studied maturational trends of EEG sleep measures in 56 healthy preterm infants (26-33 wk of gestation, normal follow-up at $24 \mathrm{mo}$ ) and demonstrated a decrease in absolute delta power during active sleep with advancing age. In the very preterm, EEG burst of slow delta waves are characteristic features which progressively disappear at 33 to $34 \mathrm{wk}$ of gestation $[13,14$, 25]. The persistence of slow delta waves after 33 to $34 \mathrm{wk}$ of gestation is related to adverse outcome [26]. These studies and the present work suggest that maturation may be assessed by changes in the lower spectral frequency band $(0.5-4 \mathrm{~Hz})$ of the EEG.

Similar to Okumura et al. [27], we observed a decrease in absolute theta power with PMA in the frontal and centrotemporal channels. This observation is in agreement with the visual interpretation of the preterm EEG. High-voltage rhythmic temporal theta bursts appear frequently at 27 to 28 wk of PMA and diminish at 34 wk of PMA or older [12-14]. These high-amplitude theta rhythms 
are characteristic of the temporal localization for very premature infants and are considered physiological [28]. A decrease in theta spectral power with increase of PMA is considered an indicator of normality, while persistence of theta spectral power after $32 \mathrm{wk}$ is associated with unfavorable neurological outcome [29].

Consistent with the article of Okumura et al. [27], we did not find significant differences in absolute spectral power changes in the alpha or beta frequency band. In contrast, others found absolute spectral power changes in the higher frequencies $(8-30 \mathrm{~Hz})$ with increase of PMA [23]. The differences in findings may be related to differences in EEG montage or selecting procedure of subsegments $[23,27]$.

As total spectral power between subjects may vary considerably, spectral values are frequently normalized for total power and expressed as relative spectral power measures. We observed a decrease in relative delta 1 power and an increase in relative delta 2 power with increase of PMA in centrotemporal channels, respectively. The relative theta power increased in the central and frontal channels. The relative alpha and beta power increased in nearly all channels. Only the relative alpha spectral power remained unchanged in the occipital channel. In general, the changes in relative spectral power are in accordance with the study of Bell et al. [23]. Other studies did not calculate relative spectral powers $[22,24,27]$.

Our observations of spectral power analysis correspond with the EEG pattern of maturing preterm infants in whom EEG change from high-amplitude low-frequency waves to low-amplitude high-frequency waves $[11,12,30]$. Total spectral power decreases with age with a shift from the lower to the higher frequency content of the EEG. The absolute and relative delta spectral power measures decrease, with increase of relative alpha and beta spectral power. As alpha and beta waves are complex EEG waveforms, these might be related to formation of connection between the cortex and subcortical nuclei and reticular formation, which mature postnatally [31].

In contrast to the majority of articles [22, 23, 27, 32-36], we studied maturational changes of spectral power analysis quan titatively and automated on whole 4 -h recordings, including several sleep-wake cycles. From a previous article using the same subjects, we observed periodic variation in background activity with alternating periods of discontinuity and continuity, suggestive for two to four sleep-wake cycles per 4-hrecording [19]. Bell et al. [23,33] performed spectral power analysis on eight 4 -s epochs in each recording. Okumura et al. [22,27] analyzed six 10-s epochs in each recording. Okumura et al. selected EEG epochs where continuous patterns with maximal expression of high-voltage slow waves of preterm infants were continuously observed, whereas Bell et al. analyzed the EEG epochs during periods of relative continuity. Paul et al. [34,36] selected 5-min periods during active and quiet sleep. Thus, analyzed epochs are small and selected by qualitative (visual) criteria that differ between studies. Remarkably, despite these differences in analysis, we found comparable spectral power data. We consider the automated method of spectral analysis on whole 4-h EEG recordings as performed in this study as highly objective, less time consuming to perform in daily clinical practice, and generating comparable data.

In general, the amount of changes in total spectral power measures with increasing PMA was higher in the centrotemporal channels than in the other channels (figure 2; table 2). This may be in agreement with imaging observations of the preterm brain, demonstrating that gyral development in parietotemporal area precedes that of other regions [37]. A correlation between some aspects 
of EEG maturation and gyration of the preterm brain is suggested [38]. With increasing PMA, the interburst interval shortened and cortical folding increased. For other spectral power measures, the correlation was less clear. Topographic mapping of changes in spectral power measures is an interesting technique to localize brain activity but is beyond the scope of this article [39].

Integrating spectral power analysis of the EEG during bedside continuous amplitude-integrated EEG monitoring in an NICU is an interesting option to assess brain maturation. For amplitudeintegrated EEG, most advocate lead placement at the biparietal areas ( $\mathrm{P}_{3}-\mathrm{P}_{4}$ channel), which overlies a vascular watershed [7]. The closest corresponding electrode pair in neonatal EEG using the 10 to 20 montage is the adjacent bicentral area, represented by $C_{3}-C_{4}$ [23]. As the spectral changes were most clearly seen in the centrotemporal channels, bilateral centrotemporal or parietotemporal channels may be valuable in continuous (spectral power) monitoring. Automated spectral power analysis of the EEG in preterm infants may provide easily accessible bedside information about brain function to the neonatologist and help to identify possible factors that interfere with normal brain development. Spectral power of low frequencies differs significantly between the burst episodes of healthy and asphyxiated infants [35]. Lower SEF measures are shown in infants with later cerebral white matter injury on MRI [40]. Less spectral beta energy is demonstrated in preterm infants who received skin-to-skin contact compared with a control group of preterm infants [41]. In preterm infants, a correlation between increased power in the higher frequency range and normal behavioral outcome at $5 \mathrm{y}$ of age is demonstrated [32]. Recently, a relation between increased delta activity and chorioamnionitis-related brain injury is demonstrated in preterm lambs [42].

There are several methodological limitations of the study. First, the number of very preterm infants is relatively small. Of 95 eligible preterm infants, 28 enrolled the study and after later exclusion of 10 (sick or delayed neurodevelopment at 2 y of age), 18 (20\%) were actually analyzed in this study. The aim of this study was to assess automated-derived spectral power EEG measures in healthy preterm infants without any additional pathologic conditions that might interfere with normal cerebral development. We consider a strength of the selection procedure that all included preterm infants showed normal neurodevelopment at $2 y$ of age and that spectral power values are obtained for "normal" preterm infants. Second, for this study, signal artifacts related to repositioning of electrodes or nursing care for the infants were removed based on an empirical impedance and spectral power threshold value. An acceptable percentage of remaining artifacts was confirmed visually. Third, our study did not address sleep stateof preterm infants, and between-state differences were not analyzed. To maximize robustness of the method, we refrained from performing analysis on subsegments, to reduce bias based on user information and to avoid possible errors in sample selection. Finally, evaluating the complex and subtle maturation aspects of the neonatal EEG requires specific neurophysiological expertise. Automated power spectral analysis must be seen as an aid in estimating neurophysiological maturation in preterm infants.

In conclusion, we performed automated spectral analysis of serial 4-h EEG recordings in 18 healthy very preterm infantswith a normal follow-up at $2 \mathrm{y}$ of age. Neurophysiological maturation of preterm infants can be demonstrated by automated spectral analysis. Maturational EEG changes assessed by amplitude spectral analysis are most prominent in the centrotemporal channels with a decrease of absolute delta frequencies $(0.5-4 \mathrm{~Hz})$. Relative spectral power measures show a shift from the lower toward the higher frequency ranges. The potential of computer-assisted analyses 
of serial EEG samples may be that using signal analytic features not readily identified visually and may provide an indicator of good prognosis or poor developmental outcome.

\section{Acknowledgments}

We thank Saskia Houterman (epidemiologist, MMC Academy, Veldhoven) for her statistical advice. 


\section{References}

1. Cooke RW. Preterm mortality and morbidity over 25 years. Arch Dis Child Fetal Neonatal Ed 2006;91(4):F293-4.

2. Larroque $B$, Ancel $P Y$, Marret $S$, Marchand $L$, Andre M, Arnaud C, et al. Neurodevelopmental disabilities and special care of 5-year-old children born before 33 weeks of gestation (the EPIPAGE study): a longitudinal cohort study. Lancet 2008;371(9615):813-20.

3. Perlman JM. Cognitive and behavioral deficits in premature graduates of intensive care. Clin Perinatol 2002;29(4):779-97.

4. Gressens $P$, Rogido $M$, Paindaveine $B$, Sola $A$. The impact of neonatal intensive care practices on the developing brain. J Pediatr 2002;140(6):646-53.

5. Volpe JJ. Electroencephalography may provide insight into timing of premature brain injury. Pediatrics 2009;124(3):e542-4.

6. Watanabe K, Hayakawa F, Okumura A. Neonatal EEG: a powerful tool in the assessment of brain damage in preterm infants. Brain Dev 1999;21(6):361-72.

7. Hellstrom-Westas L, Rosen I. Electroencephalography and brain damage in preterm infants. Early Hum Dev 2005;81(3):255-61.

8. Kidokoro H, Okumura A, Hayakawa F, Kato T, Maruyama K, Kubota T, et al. Chronologic changes in neonatal EEG findings in periventricular leukomalacia. Pediatrics 2009;124(3):e468-75.

9. Hayakawa F, Okumura A, Kato T, Kuno K, Watanabe K. Disorganized patterns: chronic-stage EEG abnormality of the late neonatal period following severely depressed EEG activities in early preterm infants. Neuropediatrics 1997;28(5):272-5.

10. Vermeulen $R J$, Sie $L T$, Jonkman EJ, Strijers $R L$, Lafeber HN, Uitdehaag BM, et al. Predictive value of EEG in neonates with periventricular leukomalacia. Dev Med Child Neurol 2003;45(9):586-90.

11. Andre M, Lamblin MD, d'Allest AM, CurziDascalova L, Moussalli-Salefranque F, TSNT, et al. Electroencephalography in premature and full-term infants. Developmental features and glossary. Neurophysiol Clin 2010;40(2):59-124.

12. ScherMS. Ontogeny of EEG sleep from neonatal through infancy periods. Handb Clin Neurol 2008;98:111-29.

13. Selton D, Andre M, Hascoet JM. Normal EEG in very premature infants: reference criteria. Clin Neurophysiol 2000;111(12):2116-24.

14. Vecchierini $M F$, d'Allest $A M$, Verpillat $P$. EEG patterns in 10 extreme premature neonates with normal neurological outcome: qualitative and quantitative data. Brain Dev 2003;25(5):330-7.

15. Scher MS, Jones BL, Steppe DA, Cork DL, Selt man HJ, Banks DL. Functional brain maturation in neonates as measured by EEG-sleep analyses. Clin Neurophysiol 2003;114(5):875-82.

16. Victor S, Appleton RE, Beirne M, Marson AG, Weindling AM. Spectral analysis of electroencephalography in premature newborn infants: normal ranges. Pediatr Res 2005;57(3):336-41.

17. West $C R$, Harding JE, Williams $C E$, Gunning MI, Battin MR. Quantitative electroencephalographic patterns in normal preterm infants over the first week after birth. Early Hum Dev 2006;82(1):43-51.

18. Niemarkt $\mathrm{HJ}$, Andriessen $P$, Peters $\mathrm{CH}$, Pasman $J W$, Blanco CE, Zimmermann LJ, et al. Quantitative analysis of amplitude-integrated electroencephalogram patterns in stable preterm infants, with normal neurological development at one year. Neonatology 2010;97(2):175-82.

19. Niemarkt $\mathrm{HJ}$, Andriessen $\mathrm{P}$, Peters $\mathrm{CH}$, Pasman $J W$, Zimmermann LJ, Bambang Oetomo S. 
Quantitative analysis of maturational changes in EEG background activity in very preterm infants with a normal neurodevelopment at 1 year of age. Early Hum Dev 2010;86(4):219-24.

20. de Vries $L S$, Eken P, Dubowitz LM. The spectrum of leukomalacia using cranial ultrasound. Behav Brain Res 1992;49(1):1-6.

21. Tekgul H, Bourgeois BF, Gauvreau K, Bergin AM. Electroencephalography in neonatal seizures: comparison of a reduced and a full 10/20 montage. Pediatr Neurol 2005;32(3):155-61.

22. Okumura A, Kubota T, Toyota N, Kidokoro H, Maruyama K, Kato T, et al. Amplitude spectral analysis of maturational changes of delta waves in preterm infants. Brain Dev 2003;25(6):406-10.

23. Bell AH, McClure BG, McCullagh PJ, McClelland $R J$. Variation in power spectral analysis of the EEG with gestational age. J Clin Neurophysiol 1991;8(3):312-9.

24. Scher MS, Steppe DA, Banks DL, Guthrie RD, Sclabassi RJ. Maturational trends of EEG-sleep measures in the healthy preterm neonate. Pediatr Neurol 1995;12(4):314-22.

25. Flores Guevara R, Giannuzzi R, Nosralla Mde $O$, Vignolo P, Moriette G, Maier MA. Positive slow waves in the EEG of premature infants between 24 and 36 weeks of conceptional age. Clin Neurophysiol 2008;119(1):180-9.

26. Hayakawa F, Okumura A, Kato T, Kuno K, Watanabe K. Dysmature EEG pattern in EEGs of preterm infants with cognitive impairment: maturation arrest caused by prolonged mild CNS depression. Brain Dev 1997;19(2):122-5.

27. Okumura A, Kubota T, Tsuji T, Kato T, Hayakawa F, Watanabe K. Amplitude spectral analysis of theta/alpha/beta waves in preterm infants. Pediatr Neurol 2006;34(1):30-4.

28. Hughes JR, Fino JJ, Hart LA. Premature temporal theta (PT theta). Electroencephalogr Clin Neurophysiol 1987;67(1):7-15.

29. Biagioni E, Bartalena L, Boldrini A, Cioni G,
Giancola S, Ipata AE. Background EEG activity in preterm infants: correlation of outcome with selected maturational features. Electroencephalogr Clin Neurophysiol 1994;91(3):154-62.

30. Vecchierini MF, Andre M, d'Allest AM. Normal EEG of premature infants born between 24 and 30 weeks gestational age: terminology, definitions and maturation aspects. Neurophysiol Clin 2007;37(5):311-23.

31. Scher MS, Turnbull J, Loparo K, Johnson MW. Automated state analyses: proposed applications to neonatal neurointensive care. J Clin Neurophysiol 2005;22(4):256-70.

32. Thordstein M, Flisberg A, Lofgren N, Bagenholm $R$, Lindecrantz K, Wallin BG, et al. Spectral analysis of burst periods in EEG from healthy and post-asphyctic full-term neonates. Clin Neurophysiol 2004;115(11):2461-6.

33. Bell AH, McClure BG, McCullagh PJ, McClelland RJ. Spectral edge frequency of the EEG in healthy neonates and variation with behavioural state. Biol Neonate 1991;60(2):69-74.

34. PaulK, Krajca V, Roth Z, Melichar J, Petranek S. Comparison of quantitative EEG characteristics of quiet and active sleep in newborns. Sleep Med 2003;4(6):543-52.

35. Richards JE, Parmelee AH, Jr., Beckwith L. Spectral analysis of infant EEG and behavioral outcome at age five. Electroencephalogr Clin Neurophysiol 1986;64(1):1-11.

36. Paul K, Krajca V, Roth Z, Melichar J, Petranek S. Quantitative topographic differentiation of the neonatal EEG. Clin Neurophysiol 2006;117(9):2050-8.

37. Dubois J, Benders M, Cachia A, Lazeyras F, Ha-Vinh Leuchter R, Sizonenko SV, et al. Mapping the early cortical folding process in the preterm newborn brain. Cereb Cortex 2008;18(6):1444-54.

38. Biagioni E, Frisone MF, Laroche S, Kapetanakis BA, Ricci D, Adeyi-Obe M, et al. Maturation of cerebral electrical activity and development of 
cortical folding in young very preterm infants.

Clin Neurophysiol 2007;118(1):53-9.

39. Pereda E, de La Cruz DM, Manas S, Garrido JM, Lopez S, Gonzalez JJ. Topography of EEG complexity in human neonates: effect of the postmenstrual age and the sleep state. Neurosci Lett 2006;394(2):152-7.

40. Inder $T E$, Buckland L, Williams $C E$, Spencer C, Gunning MI, Darlow BA, et al. Lowered electroencephalographic spectral edge frequency predicts the presence of cerebral white matter injury in premature infants. Pediatrics 2003;111(1):27-33.

41. ScherMS, Ludington-HoeS, Kaffashi F, Johnson MW, Holditch-Davis D, Loparo KA. Neurophysiologic assessment of brain maturation after an 8-week trial of skin-to-skin contact on preterm infants. Clin Neurophysiol 2009;120(10):1812-8.

42. Gavilanes AW, Gantert M, Strackx E, Zimmermann LJ, Seeldrayers S, Vles JS, et al. Increased EEG delta frequency corresponds to chorioamnionitis-related brain injury. Front Biosci (Schol Ed) 2010;2:432-8. 


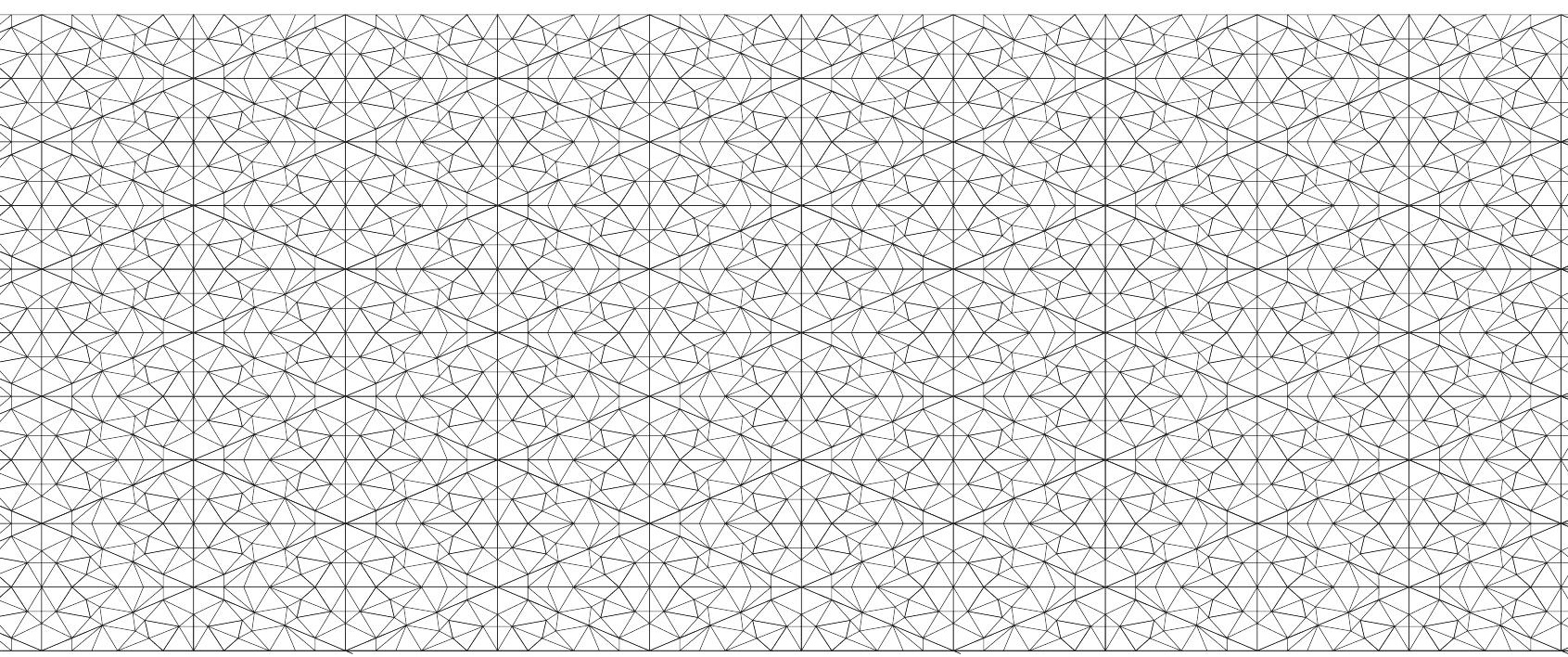




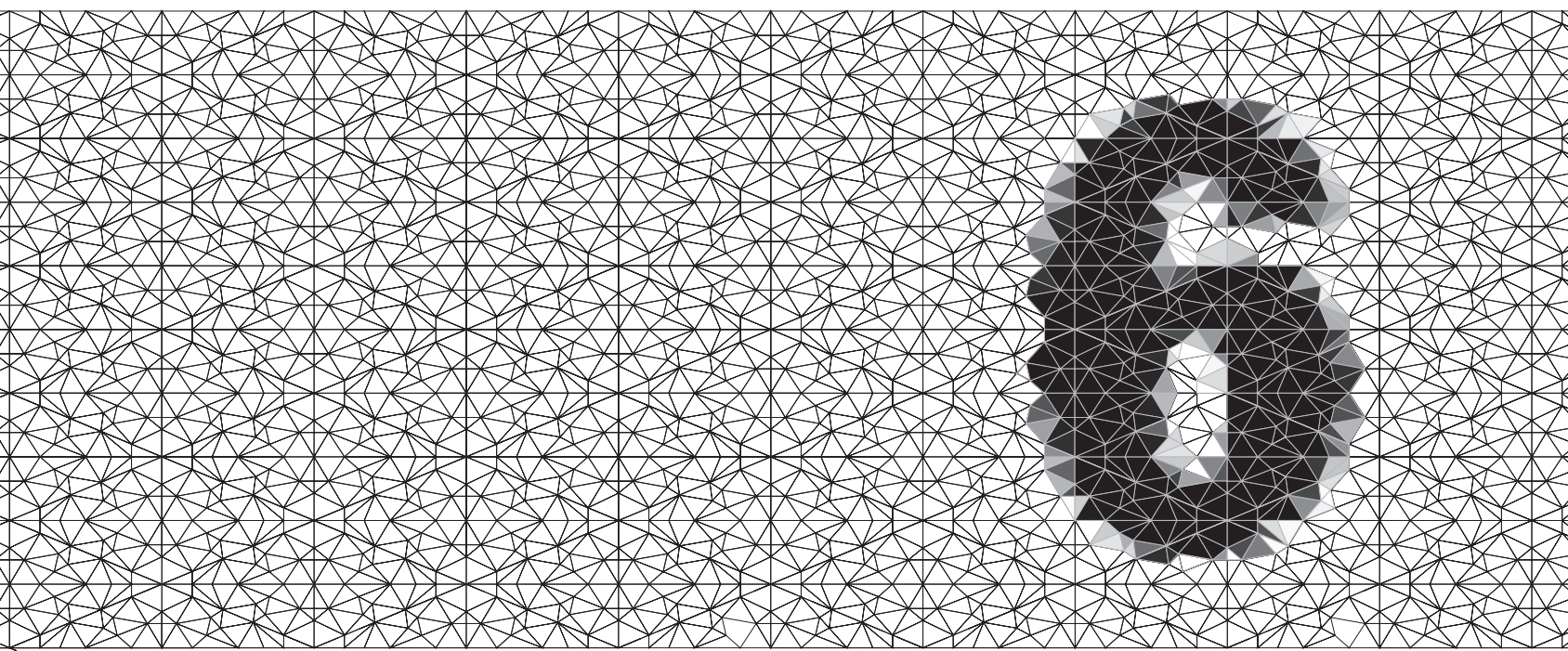

\section{Topography of maturational changes in EEG burst spectral power of the preterm infant with a normal follow-up at 2 years of age}

Ward Jennekens Hendrik J Niemarkt Marjolein Engels Jaco W Pasman Carola van Pul Peter Andriessen 


\section{Abstract}

\section{Objective}

To quantify the EEG burst frequency spectrum of preterm infants by automated analysis and to describe the topography of maturational change in spectral parameters.

\section{Methods}

18 preterm infants $<32$ weeks gestation and normal neurological follow-up at 2 years, underwent weekly 4-hour EEG recordings (10-20 system). The recordings $(n=77)$ represent a large variability in postmenstrual age (PMA, $28-36$ weeks). We applied an automated burst detection algorithm and performed spectral analysis. The frequency spectrum was divided in $\delta_{1}(0.5-1 \mathrm{~Hz}), \delta_{2}(1-4 \mathrm{~Hz}), \theta$ (4-8 $\mathrm{Hz}), a(8-13 \mathrm{~Hz})$ and $\beta(13-30 \mathrm{~Hz})$ band. Spectral parameters were evaluated as function of PMA by regression analysis. Results were interpolated and topographically visualized.

\section{Results}

The majority of spectral parameters show significant change with PMA. Highest correlation is found for $\delta$ and $\theta$ band. Absolute band powers decrease with increasing PMA, while relative $\alpha$ and $\beta$ powers increase. Maturational change is largest in frontal and temporal region.

\section{Conclusions}

Topographic distribution of maturational changes in spectral parameters corresponds with studies showing ongoing gyration and postnatal white matter maturation in frontal and temporal lobes.

\section{Significance}

Computer analysis of EEG may allow objective and reproducible analysis for long-term prognosis and/or stratification of clinical treatment. 


\section{Introduction}

Advances in neonatal intensive care of the high-risk preterm infant have led to increased survival. A significant number of these infants suffer from neurodevelopmental delays and problems in school. [1-3]. The most important determinant for future development is related to cerebrovascular injury in the (pre)term infant, cerebral hemorrhage and ischemic injury. Serial cranial ultrasound or magnetic resonance imaging may reveal parenchymal hemorrhage or white matter changes in the first weeks of life and have an important value in predicting outcome [4]. However, in the absence of cerebral lesions, the value of these imaging modalities are limited [5].

Assessing changes in electroencephalography $(E E G)$ is important in evaluating the status of the central nervous system and is useful in future prediction of outcome $[6,7]$. Although the acute and chronic EEG changes are mainly non-specific regarding type of damage, they correlate with later neurological and cognitive function $[6,8,9]$. In preterm infants developing white matter damage, acute EEG findings include decreased continuity, lower amplitude of background activity and epileptic seizure activity [10]. The chronic EEG change associated with white matter injury and abnormal neurological development including delayed maturation, is a disorganized pattern with the presence of abundant positive Rolandic sharp waves [10, 11]. Poor short-term outcome (death or severe intraventricular hemorrhage) in preterm infants is associated with absence of sleep-wake cycling [12]. Furthermore, it has been shown that spectral frequency aspects of the EEG are related to brain injury and neurodevelopmental outcome [13-15].

As the EEG has constant and reproducible patterns in normal very preterm infants, this constitutes a basis for the development of a quantitative analysis method of EEG for future neurological prognosis in preterm infants $<28$ weeks of gestation $[16,17]$. The normal preterm EEG is characterized by two main background patterns. Tracé continue comprises continuous EEG activity of similar voltage and frequency, whilst tracé discontinue comprises alternating segments of activity and quiescence which is also known as EEG discontinuity [18]. The periods of quiescence are characterized by low voltage and termed the interburst intervals (IBI). With advancing gestational age, IBI become shorter so that the proportion of time without EEG activity decreases [19, 20]. Electrocortical activity matures with increasing gestational age as seen by changes in amplitude and spectral frequencies $[16,17,20,21]$.

Generally, spectral frequency analysis of EEG is performed on selected epochs of EEG, including periods of burst and IBI [22-25]. Hence, changes in the ratio of burst and IBI with advancing age will affect the measured frequency spectrum of the EEG signal. The aim of this study was to quantify the frequency spectrum of burst activity in the EEG of healthy preterm infants, hereby excluding IBI periods. With this method, we expect to increase sensitivity for maturational changes in physiological transients of cortical activity. We performed a longitudinal study of weekly 4-hr EEG recordings in 18 preterm infants with a gestational age $<32$ weeks and a normal neurodevelopmental follow-up at 2 years of age. First, EEG bursts were selected using an automated burst detection algorithm and spectral parameters of the bursts were determined. Second, the relation between spectral parameters and postmenstrual age was analyzed. Third, the regional distribution of maturational change in spectral parameters was investigated. 


\section{Methods}

\section{Study subjects}

This study was part of a neonatal EEG research program of the Máxima Medical Centre in Veldhoven, The Netherlands, conducted in the NICU from May 2006 to July 2007. The hospital's ethics committee approved the study. Infants were enrolled after written informed consent from both parents. For details of the study group we refer to earlier papers $[19,26]$.

During the study period 449 infants were admitted to our NICU. Of these, 95 infants were eligible for the study, based on 'first-day' inclusion criteria: preterm infant with a GA $<32$ weeks, birth weight appropriate for gestational age, Apgar score $\geq 6$ at 5 minutes and arterial umbilical $\mathrm{pH}>7.00$ and normal cerebral ultrasonography (intraventricular hemorrhage grade $\leq \mathrm{I}$ ) [27]. Of the eligible population, 67 subjects were subsequently excluded in the first week because of cardiovascular instability, abnormalities on cranial ultrasound (intraventricular hemorrhage II-IV and all grades of periventricular leucomalacia) [28], use of any sedative or anti-epileptic medication, subjects with recurrent apnea treated with doxapram, or no parental consent.

Thus, 28 infants enrolled the study. Of these, 10 subjects were excluded from analysis because they met exclusion criteria after the first week or showed delayed development at 24 months of age. Finally, 18 clinically stable preterm infants with four to five serial EEG recordings and a normal neurological follow-up at 24 month of age (Bayley Scales of Infant Developmental for mental and motor function $>85$ ) were analyzed in this paper. The characteristics of these subjects are shown in table 1.

\section{Data acquisition}

Starting at the end of the first week of life, weekly digital EEG recordings (NicoletOne; Viasys Healthcare, Conshohocken, PA, USA) were performed. After skin preparation (Nuprep Gel, D.O. Weaver, Aurora, CO, USA) Ag/AgCl cup electrodes, filled with a conductive paste (Ten2O, D.O. Weaver, Aurora, $\mathrm{CO}, \mathrm{USA}$ ) were placed according the international 10-20 reduced montage system using $\mathrm{Fp}_{2}, \mathrm{Fp}_{1}, \mathrm{CZ}_{2} \mathrm{C}_{3}, \mathrm{C}_{4}, \mathrm{~T}_{3}, \mathrm{~T}_{4}, \mathrm{O}_{1}$, and $\mathrm{O}_{2}$ electrodes [29]. Impedance of the electrodes was maintained below $10 \mathrm{k} \Omega$. The digital EEG signal was sampled at $256 \mathrm{~Hz}$. Artifacts related to repositioning the infant or replacement of an electrode when impedance was above $10 \mathrm{k} \Omega$ were removed $(<5 \%$ of total data).

All recordings took place between 8-12 am and after feeding, when the infant was asleep in the incubator in prone or side position. Feeding and care for the infant was carried out according the normal routine of the NICU. The average recording time was four hours. During the measurements a video recording of the infant was made in order to relate patterns or artifacts in the EEG to the position or state of the infant.

\section{Data analysis}

Thirteen channels were used for analysis. Transverse channels: frontal Fp1-Fp2; central C3-C4; occipital $\mathrm{O}_{1}-\mathrm{O}_{2}$ and the left and right temporal $\mathrm{C}_{3}-\mathrm{T}_{3}$ and $\mathrm{C}_{4}-\mathrm{T}_{4}$. Longitudinal channels: frontaltemporal Fp1-T3 and Fp2-T4; frontal-central Fp1- $\mathrm{C}_{3}$ and $\mathrm{Fp}_{2}-\mathrm{C}_{4}$; temporal-occipital $\mathrm{T}_{3}-\mathrm{O}_{1}$ and $\mathrm{T}_{4}-\mathrm{O}_{2}$; central-occipital $\mathrm{C}_{3}-\mathrm{O}_{1}$ and $\mathrm{C}_{4}-\mathrm{O}_{2}$. 
Table 1: Characteristics of study population $(n=18)$.

\section{Clinical characteristics}

\begin{tabular}{|c|c|}
\hline Gestational age (wk) & $29.0 \pm 0,3$ \\
\hline Birth weight (g) & $1297 \pm 58$ \\
\hline Male/Female (\#, \%) & $11(61 \%) / 7(39 \%)$ \\
\hline 5-min Apgar score & $8.5 \pm 1.3$ \\
\hline Umbilical arterial pH & $7.28 \pm 0.09$ \\
\hline Tocolysis (\%) & 83 \\
\hline Antenatal corticoids (\%) & 94 \\
\hline Caesarean section (\%) & 28 \\
\hline Lowest pH, first week & $7.26 \pm 0.04$ \\
\hline Lowest glucose (mmol/l), first week & $3.0 \pm 1.1$ \\
\hline MDI at 24-mo & $106 \pm 12$ \\
\hline PDI at 24-mo & $91 \pm 11$ \\
\hline
\end{tabular}

Data were filtered using $0.5 \mathrm{~Hz}$ high-pass and $30 \mathrm{~Hz}$ low-pass $4^{\text {th }}$ order Butterworth filters. After filtering, bursts were segregated from IBI using an optimized amplitude-, time- and channelthreshold based algorithm [30]. Bursts were defined as activity with an amplitude $>30 \mu \mathrm{V}$, simultaneously occurring in $\geq 4$ channels and lasting $\leq 20 \mathrm{~s}$. Likewise, continuous activity was defined as bursts $>20 \mathrm{~s}$. Subsequently, spectral analysis of cortical activity was performed using Matlab ${ }^{\oplus}$ (The MathWorks, Massachusetts, USA). Data were divided into 4-s segments from the center of each burst. Each segment was multiplied by a Hamming window before Fourier transform. The frequency spectrum was determined for each segment, and was divided in $\delta_{1}(0.5-1 \mathrm{~Hz}), \delta_{2}(1-4$ $\mathrm{Hz}), \theta(4-8 \mathrm{~Hz}), a(8-13 \mathrm{~Hz})$ and $\beta(13-30 \mathrm{~Hz})$ band.

Three aspects of the transformed signal were calculated: (1) absolute power (defined as the integral of all powers within the frequency band, expressed in $\left.\mu \mathrm{V}^{2}\right)$, (2) relative power (defined as the ratio of absolute band power to total power of all bands, expressed in percentage); (3) spectral edge frequency (SEF) (defined as the frequency that delimits $95 \%$ of the power between $0.5-30 \mathrm{~Hz}$ ). Segments containing large artifacts were removed by applying a total power amplitude threshold, based on histogram analysis ( $<5 \%$ of data). Finally, the obtained parameters were averaged over all bursts for each channel to obtain mean burst spectral parameters per channel and per measurement.

\section{Statistical analysis}

Data with a normal distribution are expressed as a mean \pm SD, otherwise data are expressed as a median and interquartile range (IQR). Statistical analysis was performed with SPSS 13.1 (Inc, 
Table 2: Regression analysis for total power and SEF evaluated for 28 and 36 weeks PMA.

\begin{tabular}{|c|c|c|c|c|}
\hline & \multicolumn{2}{|c|}{ Total Power $(\mu \mathrm{V} 2)$} & \multicolumn{2}{|c|}{ SEF (Hz) } \\
\hline & 28 wks & 36 wks & 28 wks & 36 wks \\
\hline Frontal & $303(243-378)^{*}$ & $61(48-78)^{*}$ & $29(26-32)^{*}$ & $43(39-49)^{*}$ \\
\hline Central & $266(211-336)^{*}$ & $135(105-174)^{*}$ & $20(19-22)^{*}$ & $37(33-40)^{*}$ \\
\hline Occipital & 141 (103-192) & $113(81-157)$ & $21(19-24)$ & $26(23-29)$ \\
\hline Left CT & $533(446-638)^{*}$ & $178(146-215)^{*}$ & $22(20-24)^{*}$ & $36(33-40)^{*}$ \\
\hline Right CT & $593(513-685)^{*}$ & $165(141-192)^{*}$ & $22(20-24)^{*}$ & $37(34-41)^{*}$ \\
\hline
\end{tabular}

Legend: Regression results for total power and SEF evaluated for 28 and 36 weeks PMA. Linear regression was estimated using the random effects model, which extends the generalized linear model to allow for analysis of repeated measurements within subjects. Results are shown for frontal (Fp1-Fp1), central (C3-C4), occipital (O1-O2), left centro-temporal (C3-T3) and right centro-temporal (C4-T4) channels. Values are expressed as median with $95 \%$ confidence intervals. Significance of the regression analysis is indicated by: ${ }^{*} p<0.01 ;+p<0.05$.

Figure 1: Detection results for burst and interburst (IBI) as function of PMA.
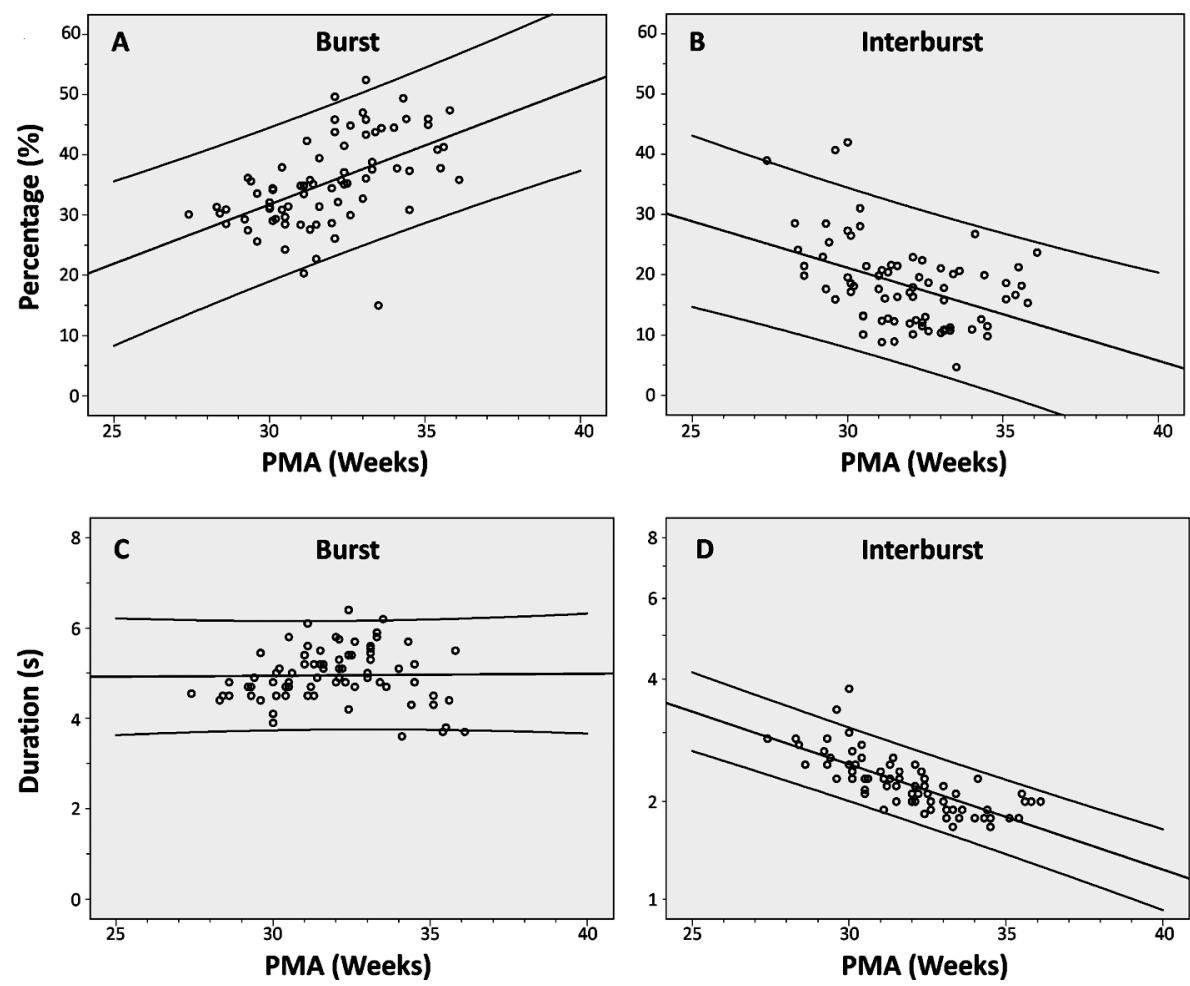

Legend: Top row shows the percentage of recording time containing detected bursts $(A)$ and IBI (B). Bottom row shows the median duration of burst (C) and IBI (D) for each measurement. Detection results are indicated by open dots. Linear regression lines are shown with $95 \%$ confidence intervals. Linear regression yields $R=0.53(p<0.01)$ for burst percentage, $R$ $=0.43(p<0.01)$ for IBI percentage, $R=0.02$ (NS) for burst duration and $R=0.76(p<0.01)$ for IBI duration. Note that the $y$-axis is logarithmic for interburst duration. 
Chicago, IL, USA). The relation between PMA and EEG spectral parameters (absolute and relative power of the different frequency bands, total power and SEF) in the different leads was evaluated. Spectral data was log-transformed, as raw spectral data had a positive skew. To correct for repeated measurements, linear regression was estimated using the random effects model, which extends the generalized linear model to allow for analysis of repeated measurements. Spectral properties estimated from linear regression models based on generalized estimating equations, can be interpreted similarly to those derived from a simple linear regression model. Regression analysis results are shown with $p$-value and $B$ coefficient (slope of regression line), and displayed with $95 \%$ confidence intervals. A p-value $<0.05$ was considered statistically significant. To visualize the regional distribution of changes in spectral parameters, statistically significant $B$ coefficients were mapped onto a 2-dimensional head model marking the reduced 10-20 system channel locations using biharmonic spline interpolation in Matlab [31].

\section{Results}

Eighteen clinically stable infants (GA $29.0 \pm 0.3 \mathrm{wk}$; birth weight $1297 \pm 58 \mathrm{~g}$; see table 1 ) underwent four to five weekly EEG recordings ( 4 recordings in $n=13$ infants; 5 recordings in $n=5$ infants). The EEG recordings $(n=77)$, representing a large variability in PMA ( $28-36$ weeks), were pooled into one dataset. All EEG recordings showed tracé continue (continuous EEG activity of similar voltage and frequency) and tracé discontinue (alternating segments of activity and quiescence) background patterns generally found in preterm infants. None of the EEG recordings showed evidence of neonatal seizures.

Applying the burst detection algorithm to the dataset resulted in 56.294 burst segments $(\leq 20$ s), 7103 continuous segments ( $20 \mathrm{~s}$ ) and $57.839 \mathrm{IBI}$ segments. The percentage of total recording time containing detected burst and IBI was correlated to PMA, as shown in figures $1 \mathrm{a}$ and $1 \mathrm{~b}$. From this figure, it can be seen that burst percentage increases with PMA, while IBI percentage decreases. The percentage of detected continuous segments was not correlated with PMA. Burst duration did not change with PMA (figure 1c). Median burst duration was 5 (IQR:3-8) s. Continuous duration was also not correlated to PMA, and median continuous duration was 32 (IQR: $25-46$ ) s. IBI duration decreased with PMA, from a median duration of $3 \mathrm{~s}$ at 27 weeks to $2 \mathrm{~s}$ at 36 weeks, as shown in figure 1d. Burst- and continuous segments with a power exceeding $10.000 \mu \mathrm{V}^{2}$ were considered artifacts ( $<5 \%$ of data) and therefore excluded from further analysis.

After log-transformation of burst spectral parameters, linear regression was performed using a random effects model to correct for repeated measurements. A total of 156 variables (12 spectral parameters for 13 channels) were analyzed. Of these variables, 115 (74\%) showed a significant correlation. Tables 2 and 3 shows the results of our regression analysis for total power, SEF and absolute band powers, evaluated for 28 and 36 weeks PMA. Regression analysis of total power as function of PMA is shown for all 13 observed channels in figure 2. For each channel, regression plots are placed according to (anatomical) position as defined by the international 10-20 system. This figure clearly demonstrates that the slope of the regression line, indicating change of total power, depends on channel position. Subsequently, we interpolated the slopes (indicating change 


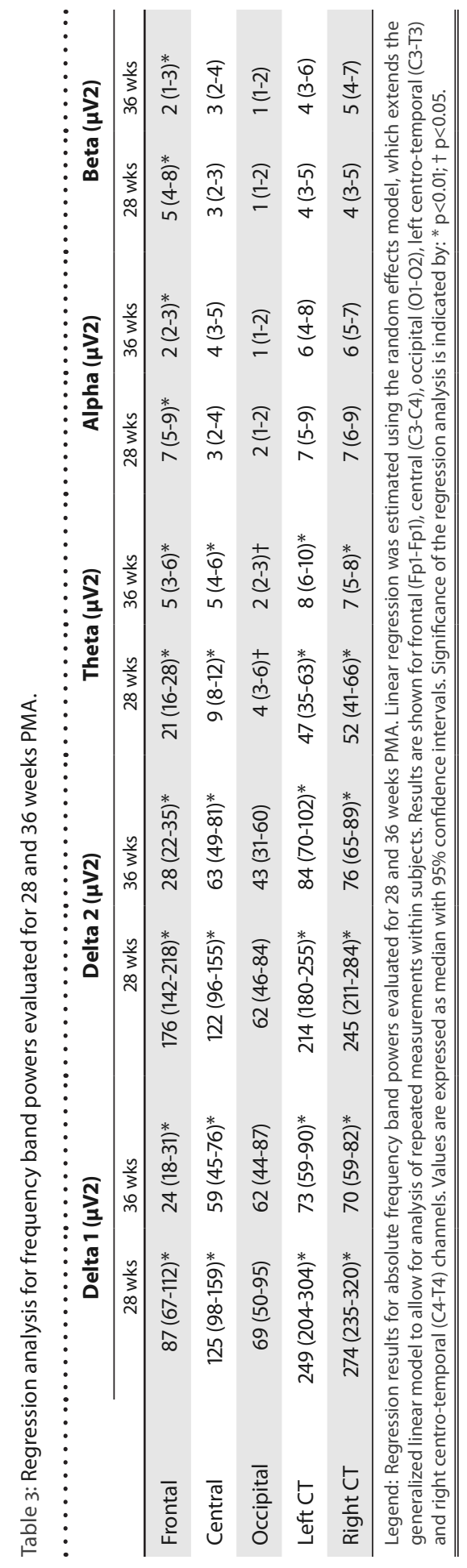

Figure 3: Topographic distribution of change in total burst power.

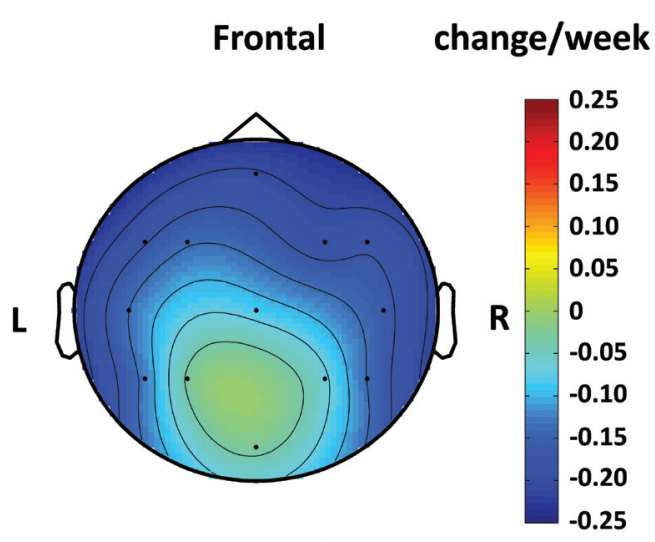

\section{Occipital}

Legend: Interpolated change in total power per week (i.e. slope of regression lines, log total power change per week, expressed in week- ${ }^{-1}$, the magnitude of change is indicated by the vertical color bar (red = positive, blue = negative change). Dark blue areas indicate the largest changes. Note that all observed total power changes are negative, i.e. total power decreases with PMA.

Figure 4: Topographic distribution of change in burst spectral edge frequency.

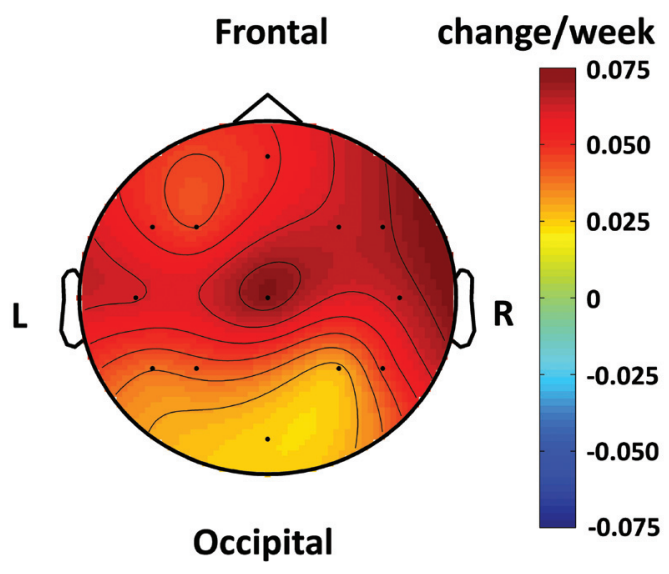

Legend: Interpolated change in spectral edge frequency (SEF) per week (log SEF change per week, expressed in week-1), the magnitude of change is indicated by the vertical color bar (red = positive, blue = negative change). Red areas indicate the largest changes. Note that all observed SEF changes are positive, i.e. SEF increases with PMA. 
Figure 2: Regression plots for total burst power as function of PMA, placed according to international 10-20 system.

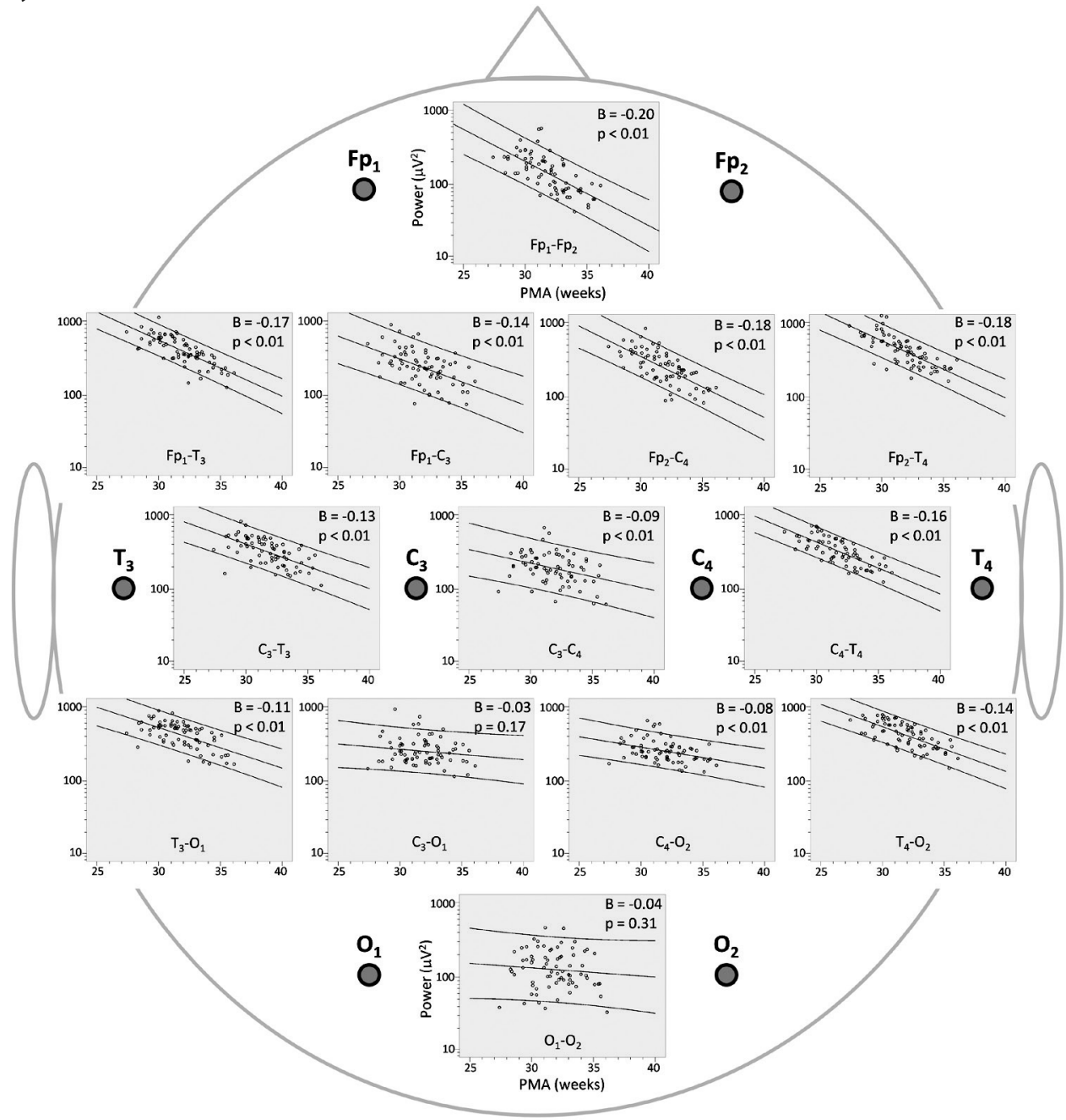

Legend: The relationship between absolute postmenstrual age (PMA, $x$-axis, weeks) and total burst power $\left(y\right.$-axis, $\left.\mu V^{2}\right)$ is shown for all 13 channels of the reduced 10-20 system (electrode positions are indicated by the labeled gray dots). Linear regression line is shown with $95 \%$ confidence intervals. Note that the $x$-axis is linear and the $y$-axis is logarithmic. For each channel, the slope of the regression line (B) and the significance level ( $p$ ) are indicated.

per week) within the 10-20 system and visualized the results in a topographical distribution (figure 3). From this figure, it can be seen that the decrease in total power is largest in the frontal and temporal regions. Likewise, this analysis method was used to visualize the change per week for all burst spectral parameters. 
Figure 5: Topographic distribution of change in absolute burst spectral power.
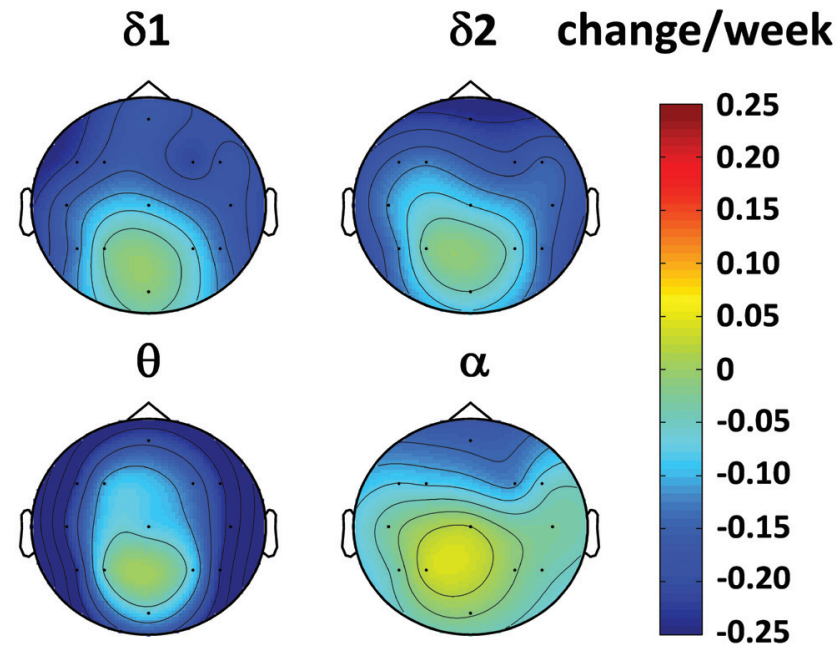

Legend: Interpolated change in absolute band power per week (i.e. slope of regression lines, log absolute power change per week, expressed in week-1), the magnitude of change is indicated by the vertical color bar (red = positive, blue = negative change). The $\beta$ band power showed no significant correlation, and was therefore omitted from this figure. Dark blue areas indicate the largest changes.

Figure 6: Topographic distribution of change in relative burst spectral power.
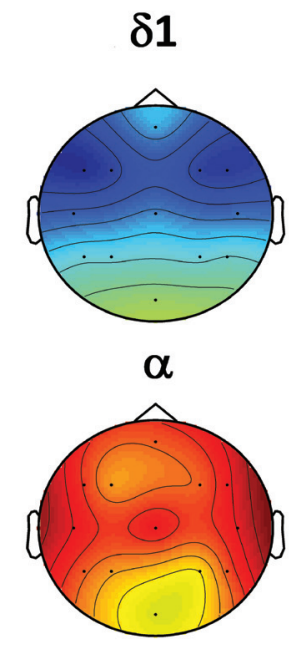

$\delta 2$ change/week
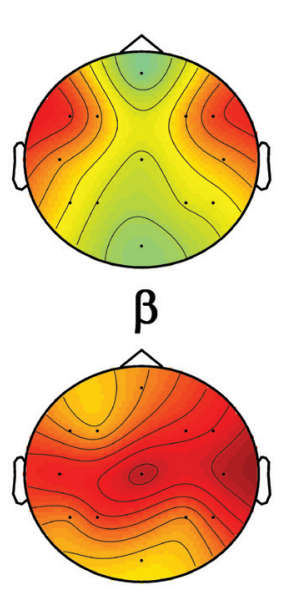

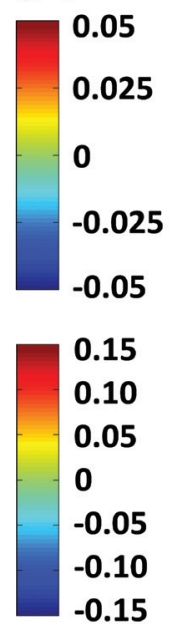

Legend: Interpolated change in relative band power per week (i.e. slope of regression lines, log relative spectral power, expressed in week-1), indicated by the vertical color bars ( $r e d=$ positive, blue = negative change). Note that the top color bar indicates the change in $\delta 1$ and $\delta 2$, and the bottom color bar, using a different scale, indicates the change in $a$ and $\beta$ band. Also note that the relative $\theta$ band power showed no significant correlation, and was therefore omitted from this figure. Dark blue and dark red areas indicate the largest changes. 


\section{Spectral edge frequency analysis}

SEF increased with PMA in all channels (figure 4). The largest changes are observed in the centrotemporal regions.

\section{Absolute spectral power analysis}

Figure 5 shows the result for absolute $\delta_{1}, \delta_{2}, \theta$ and band powers. The absolute a band power showed no significant correlation, and was therefore omitted from this figure. Absolute spectral band powers decrease with PMA. The largest changes are observed in the fronto-temporal regions.

\section{Relative spectral power analysis}

Figure 6 shows the result for relative $\delta_{1}, \delta_{2}$, $a$ and $\beta$ band powers. The relative $\theta$ band power showed no significant correlation, and was therefore omitted from this figure. Relative $\delta_{1}$ band powers decreases with PMA while relative $\delta 2, a$ and $\beta$ power increase with PMA. The largest changes are observed in the fronto-temporal regions.

\section{Discussion}

An important methodological distinction between our study and other studies describing maturation of the EEG is the focus on analysis of burst activity. Generally, spectral analysis of EEG activity is performed on selected epochs of EEG which include periods of burst and IBI [22-25]. For the infant younger than 30 weeks PMA, most recordings will consist of discontinuous EEG [32]. The percentage of discontinuous activity decreases with increasing PMA [33, 34]. Changes in the ratio of burst and IBI with advancing age will affect the measured frequency spectrum of the EEG signal. By only selecting periods of burst activity for analysis, using objective selection criteria, this bias is prevented. The burst detection algorithm used for this study is based on burst amplitude, channel and time thresholds[30]. A second distinction is the inclusion of longitudinal observations, i.e. weekly EEG measurements for each infant, whereas most studies describing maturation of cerebral activity measure each infant on only one occasion [22, 24, 25, 35-37]. With these data, both the inter- and intra-individual variation and change can be statistically accounted for. To include these repeated measurements in the linear regression we applied the random effects model, a multi-level statistical method which extends the generalized linear model to allow for analysis of repeated measurements. Finally, we visualized the regional distribution of change in burst spectral power with age, i.e. a measure for the rate of maturation, in contrast with other studies directly mapping EEG spectral properties [36, 38].

By selecting only burst periods for analysis, we expected to increase sensitivity for maturational changes in cortical activity transients compared to non-specific analysis. Referring to our previous publication [23], comparison of both methods indeed shows higher significance levels for total power in the central channel and theta power in the frontal, central and temporal channels. Significance levels resulting for regression analysis for the other spectral parameters were comparable between methods. Spectral information from the total data set was normally distributed, while spectral information from the isolated burst activity required logarithmic transformation before analysis. 
In general, maturation of electro-cortical activity is characterized by 1 ) increasing continuity and 2) changes in morphology of burst activity, i.e. amplitude and spectral power and 3) organization of sleep-states $[20,39]$. This study focused on changes in continuity and morphology of EEG activity.

\section{Changes in EEG continuity}

In our study, continuity of cortical activity increased with PMA. The percentage of recording time containing detected bursts increased from $25 \%$ at 27 weeks to $40 \%$ at 36 weeks, while the IBI percentage was shown to decrease from $25 \%$ at 27 weeks to $10 \%$ at 36 weeks. Comparable changes were observed by Hahn et al. [40] and Victor et al. [37]. Hahn showed the percentage of continuous activity, i.e. EEG without IBI, to increase from approximately $20 \%$ at 30 weeks to $40 \%$ at 37 weeks. The IBI percentage decreases from $10 \%$ at 26 weeks to $<1 \%$ at 37 weeks. These very low IBI percentages may be explained by the strict IBI definition applied by Hahn $(<15 \mu \mathrm{V}$ and $\geq 2 \mathrm{~S}$ as opposed to $<30 \mu \mathrm{V}$ and $\geq 1 \mathrm{~s}$ in our study). Victor et al. found the IBI percentage to decrease from $54 \%$ at 26 weeks to $19 \%$ at 30 weeks, using the same amplitude criterion as in our study, however without including a minimum IBI duration.

We observed no significant relation between burst duration and PMA for the total study population, with a median burst duration of $5(\mathrm{IQR}: 3-8) \mathrm{s}$. These findings are in agreement with Anderson et al. [33]. In contrast, other authors observed increasing burst duration with PMA [20, 35]. As the burst detection algorithm used in our study was validated for a PMA range of 29-34 weeks [30], we analyzed a subset of our data with PMA $<34$ weeks. In this subset, we found the median burst duration to increase from $4 \mathrm{~s}$ at 27 weeks PMA to $6 \mathrm{~s}$ at 34 weeks PMA. From figure $1 \mathrm{c}$, burst duration can be seen to decrease for PMA > 34 weeks, explaining the regression results for the total PMA range. Examining detection results shows an increase in undefined segments starting at 34 weeks (from approximately $10 \%$ to $25 \%$ ), which may be explained by maturational changes in burst amplitude unaccounted for in detection thresholds of the burst detection algorithm.

IBI duration decreased with PMA, from a median duration of $3 \mathrm{~s}$ at 27 weeks to $2 \mathrm{~s}$ at 36 weeks, consistent with other studies $[20,33,35,37,40]$. Hahn et al. showed a decrease from approximately $4 \mathrm{~s}$ at 26-27 weeks to $2 \mathrm{~s}$ at term (38-40 weeks). Biagioni similarly demonstrated a decreasing maximum IBI duration, from $31 \mathrm{~s}$ at 27 weeks to $15 \mathrm{~s}$ at 33 weeks. Victor and Scher did not provide quantitative values.

\section{Changes in burst morphology}

In this study, we quantified changes in burst morphology through amplitude (total burst power) and spectral parameters (superimposed activity). Spectral burst parameters consist of absolute and relative frequency band power, and the SEF.

Total burst power decreased in all - except the occipital - channels, with maximum changes in fronto-temporal region. Total power decreases from approximately $300 \mu \mathrm{V}^{2}$ at 28 weeks to $100 \mu \mathrm{V}^{2}$ at 35 weeks in this region. These changes are in accordance with Scher et al. and Wallois [41, 42].

Absolute power in the delta-1 and delta-2 frequencies decreased in all - except the occipital channels. These changes are comparable to the findings of other studies which analyze random sub-segments of data [22, 24, 43] , or complete EEG recordings [23]. In contrast, we focused of physiological transients of EEG activity. Biagioni showed a decreasing maximum delta wave 
amplitude, which is directly related to delta power, between 27 and 34 weeks PMA [35]. We observed a decrease in absolute theta power with PMA in the frontal and both temporal channels, which is partly in agreement with Okumura et al. who found a decrease in absolute theta power in all channels [24]. Changes in theta power may be attributed to temporal saw-tooth waveforms, i.e. rhythmic bursts of sharp theta activity. These waves typically appear at 26 weeks, attain their maximum at 29-30 weeks PMA and disappear at 32 weeks PMA. The temporal waves in the occipital region (26-28 weeks PMA) precede the temporal wave in the temporal region and seem to mature earlier (decrease in amplitude and increase in frequency) [39]. We observed a decrease in absolute alpha power with PMA in the frontal region, in agreement with Okumura et al. Finally, we found no significant (linear) correlation between absolute beta power and PMA. After extending our procedure to include non-linear analysis, we observed a quadratic relationship between beta power and PMA, in accordance with Bell et al. [22]. This may indicate maturation of complex (non-linear) inter-neuronal networks. Bell et al. [22] observed this development to coincide with the formation of connections between subcortical nuclei and reticular formation with the cerebral cortex.

Relative delta-1 power decreased and relative delta-2 power increased with PMA in the central and temporal channels. Relative alpha power increased in all - except the occipital - channels and relative beta power increased in all channels with a maximum increase in the centrotemporal region. These observations are in accordance with results by Bell et al. [22]. The increase of relative alpha and beta power may be attributed to development of delta-brush waveforms, i.e. delta waves with superimposed rapid alpha and beta activity, usually on the ascending slope. These specific waveforms are first observed around 26 weeks PMA in vertex and central region, and increase in number until 32 weeks PMA, when they are observed in temporal and occipital regions [39, 43, 44].

SEF increased with PMA in all leads, with the largest change in centro-temporal region and the smallest change in the occipital region, indicating a shift towards higher frequencies in accordance with other studies[45, 46]. Observed changes in SEF are consistent with changes in absolute and relative band power; the SEF changes due to an increase in relative alpha and beta power.

\section{Topographic mapping of burst maturation}

The visualization of EEG signals by means of topographic mapping was first described by Ueno and Matsuoka [47] and Duffy et al [48]. Ishiwa et al. applied topographic mapping to determine the distribution of EEG power, and its age dependency, during quiet and active sleep in preterm infants [36]. Mandelbaum et al. demonstrated the feasibility of applying topographic EEG mapping to reliably identify neurological injury in newborns [38]. Our study differs from previous topographic mapping studies by determining the regional distribution of change in burst spectral power with age, i.e. a measure for the rate of maturation, as opposed to directly mapping EEG spectral properties.

Overall, we observed the largest maturational changes in the frontal and temporal regions, while the smallest changes were seen in the central and occipital region. For preterm infants $<30$ weeks PMA, cortical activity predominates in the vertex, central and occipital regions. In addition, a relative quiescence of temporal EEG activity was noted, and may reflect underdevelopment of frontal and temporal regions of the brain [43]. From these observations, for infants $>30$ weeks PMA the largest postnatal maturational changes are expected in frontal and temporal regions. Biagioni et al. showed a strong correlation between burst-related maturational features of the EEG and 
cortical folding [49]. Topographic distribution of maturational changes for investigated spectral frequency parameters as found in our study are consistent with the timeline of cerebral gyration, where cortical folding begins in the central region, and progresses in occipito-rostral direction [50]. Battin et al. demonstrated that gyral development in the central area (sulcus) precedes that of other regions, with the anterior temporal region being the least well developed [51]. Our findings also correspond with MRI studies of white matter development, showing ongoing postnatal maturation in the frontal and temporal lobes [52]. From 28 weeks onwards, organizational events occur in the cerebral cortex with alignment of neurons, development of dendrites and the formation of synapses[53-55]. Additionally, Yakolev and Lecours demonstrated myelination progressing more rapidly in the forebrain after birth [56].

\section{Limitations}

First, the number of infants included in our study is relatively low. However, the aim of this study was to assess automated EEG analysis in a cohort of healthy preterm infants without pathologic conditions that might interfere with normal cerebral development. All preterm infants showed normal neurodevelopment after 2 years of follow-up. Despite the relatively low number of infants included, the majority of the spectral parameters show significant change with PMA.

Second, the possible inclusion of artifacts in the analysis may alter the determined frequency spectra. Artifacts related to repositioning the infant or replacement of an electrode when impedance was above $10 \mathrm{k} \Omega$ were removed. Remaining large artifacts were removed using an amplitude threshold $\left(10.000 \mu \mathrm{V}^{2}\right)$ based on histogram analysis and evaluation of corresponding high amplitude outliers. In total, less than $5 \%$ of the data was considered to contain artifacts. As we did not want to interfere with the automated nature of the study, and intended to study long recordings, we did not scrutinize the signal for small artifacts. The contribution of remaining artifacts is assumed to be independent of PMA, and will therefore not introduce changes in observed trends.

Third, burst length was not taken into account for frequency analysis. Partial inclusion of interburst periods within the analysis window might be possible in our study. IBI have lower power and a different frequency content compared to burst periods [57,58], which could lead to changes in determined power spectra. As continuity increases with PMA [32], the influence of the interburst periods on measured burst power spectra will decrease with PMA. A possible solution would be to obtain a spectral estimate for burst periods of short duration through an autoregressive method, e.g. Yule-Walker [59].

Finally, in this study we did not differentiate between sleep-states for determination of spectral powers. The 4-hour datasets contains on average 2 to 4 sleep-wake cycles, as determined previously [19]. To maximize robustness and minimize selection bias, we performed automated analysis of maturational EEG features obtained from the complete recordings, with the exception of previously defined artifact periods.

In conclusion, we performed automated and quantitative spectral analysis of serial EEG recordings in 18 healthy premature infants with a normal follow-up at 2 years of age. The EEG activity of preterm infants as seen in our study changes with increasing age. It starts with mainly discontinuous high-power, low-frequency activity and, as age progresses, the EEG becomes progressively more continuous and power decreases over the entire frequency range. As low-frequency components 
decrease most, there is a relative shift towards higher frequencies. This effect also leads to an increased SEF. The changes in the EEG frequency spectrum may be caused by the development of neuronal connectivity in the brain, increasing network complexity [53-55]. Additionally, analysis of bursts in the EEG signal showed the topographic distribution of maturational changes in the investigated spectral frequency parameters. These novel findings correspond with MRI studies showing ongoing postnatal gyration and white matter maturation in the frontal and temporal lobes. With reference values for the topography of maturational EEG changes in preterm infants with normal follow-up, effects of developmental delay or perinatal asphyxia may become detectable and quantifiable in an early stage. Computer analysis of EEG may then allow objective and reproducible patterns for long-term prognosis and/or stratification of clinical treatment.

\section{Acknowledgements}

We thank Saskia Houterman (Epidemiologist, MMC Academy, Veldhoven, The Netherlands) for her statistical advice. No financial support was from extramural sources. 


\section{References}

1. Doyle LW. Evaluation of neonatal intensive care for extremely low birth weight infants in Victoria over two decades: I. Effectiveness. Pediatrics 2004;113(3 Pt 1):505-9.

2. Wilson-Costello D, Friedman $H$, Minich $N$, Fanaroff AA, Hack M. Improved survival rates with increased neurodevelopmental disability for extremely low birth weight infants in the 1990s. Pediatrics 2005;115(4):997-1003.

3. Wilson-Costello D, Friedman H, Minich $N$, Siner B, Taylor G, Schluchter M, et al. Improved neurodevelopmental outcomes for extremely low birth weight infants in 2000-2002. Pediatrics 2007;119(1):37-45.

4. Horsch S, Skiold B, Hallberg B, Nordell B, Nordell $A$, Mosskin M, et al. Cranial ultrasound and MRI at term age in extremely preterm infants. Arch Dis Child Fetal Neonatal Ed 2010;95(5):F310-4.

5. Leijser LM, de Bruine FT, van der Grond J, Steggerda SJ, Walther FJ, van Wezel-Meijler $G$. Is sequential cranial ultrasound reliable for detection of white matter injury in very preterm infants? Neuroradiology 2010;52(5):397-406.

6. Hellstrom-Westas $L$, Rosen I. Electroencephalography and brain damage in preterm infants. Early Hum Dev 2005;81(3):255-61.

7. West $C R$, Harding JE, Williams $C E$, Gunning MI, Battin MR. Quantitative electroencephalographic patterns in normal preterm infants over the first week after birth. Early Hum Dev 2006;82(1):43-51.

8. Hayakawa F, Okumura A, Kato T, Kuno K, Watanabe K. Disorganized patterns: chronic-stage EEG abnormality of the late neonatal period following severely depressed EEG activities in early preterm infants. Neuropediatrics 1997;28(5):272-5.

9. Watanabe K, Hayakawa F, Okumura A. Neonatal EEG: a powerful tool in the assessment of brain damage in preterm infants. Brain Dev
1999:21(6):361-72.

10. Kidokoro H, Okumura A, Hayakawa F, Kato T, Maruyama K, Kubota T, et al. Chronologic changes in neonatal EEG findings in periventricular leukomalacia. Pediatrics 2009;124(3):e468-75.

11. Vermeulen $R J$, Sie $L T$, Jonkman EJ, Strijers $R L$, Lafeber HN, Uitdehaag BM, et al. Predictive value of EEG in neonates with periventricular leukomalacia. Dev Med Child Neurol 2003;45(9):586-90.

12. Bowen $J R$, Paradisis $M$, Shah D. Decreased aEEG continuity and baseline variability in the first 48 hours of life associated with poor short-term outcome in neonates born before 29 weeks gestation. Pediatr Res 2010;67(5):538-44.

13. Gavilanes AW, Gantert M, Strackx E, Zimmermann LJ, Seeldrayers S, Vles JS, et al. Increased EEG delta frequency corresponds to chorioamnionitis-related brain injury. Front Biosci (Schol Ed) 2010;2:432-8.

14. Richards JE, Parmelee AH, Jr., Beckwith L. Spectral analysis of infant EEG and behavioral outcome at age five. Electroencephalogr Clin Neurophysiol 1986;64(1):1-11.

15. Thordstein M, Flisberg A, Lofgren N, Bagenholm $R$, Lindecrantz $K$, Wallin $B G$, et al. Spectral analysis of burst periods in EEG from healthy and post-asphyctic full-term neonates. Clin Neurophysiol 2004;115(11):2461-6.

16. Selton D, Andre M, Hascoet JM. Normal EEG in very premature infants: reference criteria. Clin Neurophysiol 2000;111(12):2116-24.

17. Vecchierini MF, d'Allest AM, Verpillat P. EEG patterns in 10 extreme premature neonates with normal neurological outcome: qualitative and quantitative data. Brain Dev 2003;25(5):330-7.

18. Holmes GL, Lombroso CT. Prognostic value of background patterns in the neonatal EEG. J Clin Neurophysiol 1993;10(3):323-52. 
19. Niemarkt HJ, Andriessen P, Peters CH, Pasman JW, Zimmermann LJ, Bambang Oetomo S. Quantitative analysis of maturational changes in EEG background activity in very preterm infants with a normal neurodevelopment at 1 year of age. Early Hum Dev 2010;86(4):219-24.

20. Vecchierini MF, Andre M, d'Allest AM. Normal EEG of premature infants born between 24 and 30 weeks gestational age: terminology, definitions and maturation aspects. Neurophysiol Clin 2007;37(5):311-23.

21. Selton $D$, Andre $M$, Debruille $C$, Deforge $H$, Fresson J, Hascoet JM. EEG at 6 weeks of life in very premature neonates. Clin Neurophysiol 2010;121(6):818-22.

22. Bell AH, McClure BG, McCullagh PJ, McClelland $R J$. Variation in power spectral analysis of the EEG with gestational age. J Clin Neurophysiol 1991;8(3):312-9.

23. Niemarkt HJ, Jennekens W, Pasman JW, Katgert T, Van Pul C, Gavilanes AW, et al. Maturational changes in automated EEG spectral power analysis in preterm infants. Pediatr Res 2011;70(5):529-34.

24. Okumura A, Kubota T, Toyota N, Kidokoro H, Maruyama K, Kato T, et al. Amplitude spectral analysis of maturational changes of delta waves in preterm infants. Brain Dev 2003;25(6):406-10.

25. Okumura A, Kubota T, Tsuji T, Kato T, Hayakawa F, Watanabe K. Amplitude spectral analysis of theta/alpha/beta waves in preterm infants. Pediatr Neurol 2006;34(1):30-4.

26. Niemarkt HJ, Andriessen P, Peters CH, Pasman JW, Blanco CE, Zimmermann LJ, et al. Quantitative analysis of amplitude-integrated electroencephalogram patterns in stable preterm infants, with normal neurological development at one year. Neonatology 2010;97(2):175-82.

27. Papile LA, Burstein J, Burstein R, Koffler $H$. Incidence and evolution of subependymal and intraventricular hemorrhage: a study of infants with birth weights less than 1,500 gm. J Pediatr 1978;92(4):529-34.

28. de Vries $L S$, Eken $P$, Dubowitz LM. The spectrum of leukomalacia using cranial ultrasound. Behav Brain Res 1992;49(1):1-6.

29. Tekgul H, Bourgeois BF, Gauvreau K, Bergin AM. Electroencephalography in neonatal seizures: comparison of a reduced and a full 10/20 montage. Pediatr Neurol 2005;32(3):155-61.

30. Jennekens $W$, Ruijs LS, Lommen CM, Niemarkt HJ, Pasman JW, van Kranen-Mastenbroek $\mathrm{VH}$, et al. Automatic burst detection for the EEG of the preterm infant. Physiol Meas 2011;32(10):1623-37.

31. Sandwell DT. Biharmonic spline interpolation of GEOS-3 and SEASAT altimeter data. Geophys Res Lett 1987;14(2):139-42.

32. Herbertz S, Pulzer F, Gebauer C, Panhofer M, Robel-Tillig E, Knupfer M. The effect of maturation and sedation on amplitude-integrated electroencephalogram of the preterm neonate: results of a prospective study. Acta Paediatr 2006;95(11):1394-9.

33. Anderson CM, Torres F, Faoro A. The EEG of the early premature. Electroencephalogr Clin Neurophysiol 1985;60(2):95-105.

34. Van Sweden B, Koenderink M, Windau G, Van de Bor M, Van Bel F, Van Dijk JG, et al. Long-term EEG monitoring in the early premature: developmental and chronobiological aspects. Electroencephalogr Clin Neurophysiol 1991;79(2):94-100.

35. Biagioni E, Bartalena L, Boldrini A, Cioni G, Giancola S, Ipata AE. Background EEG activity in preterm infants: correlation of outcome with selected maturational features. Electroencephalogr Clin Neurophysiol 1994;91(3):154-62.

36. Ishiwa S, Ogawa T, Sonoda H. Developmental characteristics of topographic EEG in the newborn using an autoregressive model. Brain Topogr 1991;4(1):23-30.

37. Victor S, Appleton RE, Beirne M, Marson AG, 
Weindling AM. Spectral analysis of electroencephalography in premature newborn infants: normal ranges. Pediatr Res 2005;57(3):336-41.

38. Mandelbaum DE, Krawciw N, Assing E, Ostfeld B, Washburn D, Rosenfeld D, et al. Topographic mapping of brain potentials in the newborn infant: the establishment of normal values and utility in assessing infants with neurological injury. Acta Paediatr 2000;89(9):1104-10.

39. Niemarkt HJ, Andriessen P, Pasman J, Vles JS, Zimmermann LJ, Bambang Oetomo S. Analyzing EEG maturation in preterm infants: the value of a quantitative approach. J PerinatNeonatal Med 2008;1(3):131-44.

40. Hahn JS, Monyer H, Tharp BR. Interburst interval measurements in the EEGs of premature infants with normal neurological outcome. Electroencephalogr Clin Neurophysiol 1989;73(5):410-8.

41. Scher MS, Steppe DA, Banks DL, Guthrie RD, Sclabassi RJ. Maturational trends of EEG-sleep measures in the healthy preterm neonate. Pediatr Neurol 1995;12(4):314-22.

42. Wallois F. Synopsis of maturation of specific features in EEG of premature neonates. Neurophysiol Clin 2010;40(2):125-6.

43. Scher MS. Normal electrographic-polysomnographic patterns in preterm and fullterm infants. Semin Pediatr Neurol 1996;3(1):2-12.

44. Andre $M$, Lamblin MD, d'Allest AM, CurziDascalova L, Moussalli-Salefranque F, TSNT, et al. Electroencephalography in premature and full-term infants. Developmental features and glossary. Neurophysiol Clin 2010;40(2):59-124.

45. Bell AH, McClure BG, McCullagh PJ, McClelland $R J$. Spectral edge frequency of the EEG in healthy neonates and variation with behavioural state. Biol Neonate 1991;60(2):69-74.

46. Szeto HH. Spectral edge frequency as a simple quantitative measure of the maturation of electrocortical activity. Pediatr Res 1990;27(3):289-92.
47. Ueno S, Matsuoka S. Topographic display of slow wave types of EEG abnormality in patients with brain lesions. Jpn Med Electr Bio-Eng 1976;14:118-25.

48. Duffy FH, Burchfiel JL, Lombroso CT. Brain electrical activity mapping (BEAM): a method for extending the clinical utility of EEG and evoked potential data. Ann Neurol 1979;5(4):309-21.

49. Biagioni E, Frisone MF, Laroche S, Kapetanakis BA, Ricci D, Adeyi-Obe M, et al. Maturation of cerebral electrical activity and development of cortical folding in young very preterm infants. Clin Neurophysiol 2007;118(1):53-9.

50. Dubois J, Benders M, Cachia A, Lazeyras F, Ha-Vinh Leuchter R, Sizonenko SV, et al. Mapping the early cortical folding process in the preterm newborn brain. Cereb Cortex 2008;18(6):1444-54.

51. Battin MR, Maalouf EF, Counsell SJ, Herlihy AH, Rutherford MA, Azzopardi D, et al. Magnetic resonance imaging of the brain in very preterm infants: visualization of the germinal matrix, early myelination, and cortical folding. Pediatrics 1998;101(6):957-62.

52. Dudink J, Lequin $M$, van Pul C, Buijs J, Conneman N, van Goudoever J, et al. Fractional anisotropy in white matter tracts of very-low-birth-weight infants. Pediatr Radiol 2007;37(12):1216-23.

53. Molliver ME, Kostovic I, van der Loos H. The development of synapses in cerebral cortex of the human fetus. Brain Res 1973;50(2):403-7.

54. Purpura DP. Dendritic differentiation in human cerebral cortex: normal and aberrant developmental patterns. Adv Neurol 1975;12:91-134.

55. Volpe JJ. Neurology of the newborn. 5th Edition. Philadelphia: Elsevier; 2008.

56. Yakovlev PI, A.R. L. The myelogenetic cycle of regional maturation of the brain. In: Yakovlev PI, Lecours AR, editors. Regional development of the brain in early life. Oxford: Blackwell; 1967. p. 3-70. 
57. Eiselt M, Schendel M, Witte H, Dorschel J, CurziDascalova L, D'Allest AM, et al. Quantitative analysis of discontinuous EEG in premature and full-term newborns during quiet sleep. Electroencephalogr Clin Neurophysiol 1997;103(5):528-34.

58. Palmu K, Wikstrom S, Hippelainen E, Boylan $G$, Hellstrom-Westas L, Vanhatalo S. Detection of 'EEG bursts' in the early preterm EEG: visual vs. automated detection. Clin Neurophysiol 2010;121(7):1015-22.

59. Pardey J, Roberts S, Tarassenko L. A review of parametric modelling techniques for EEG analysis. Med Eng Phys 1996;18(1):2-11. 



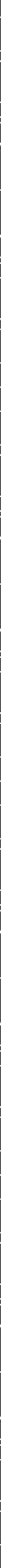




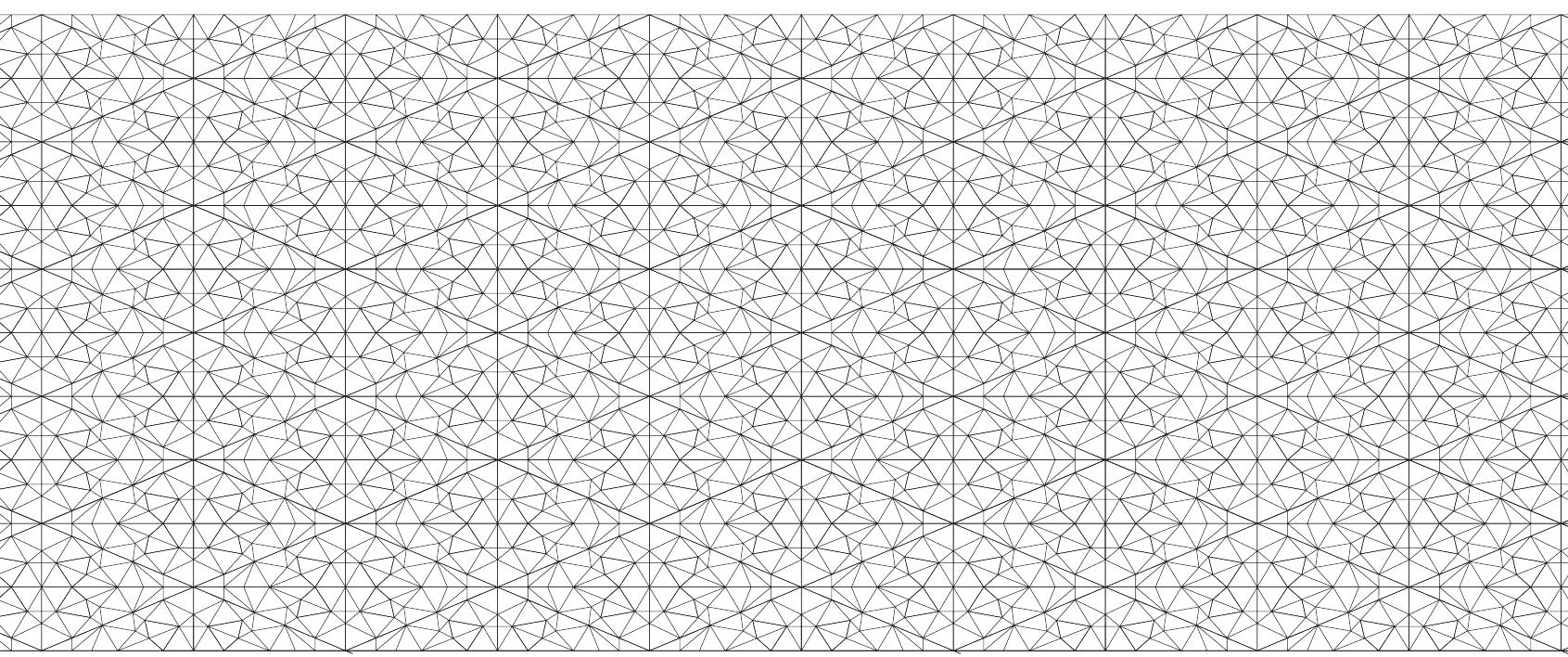




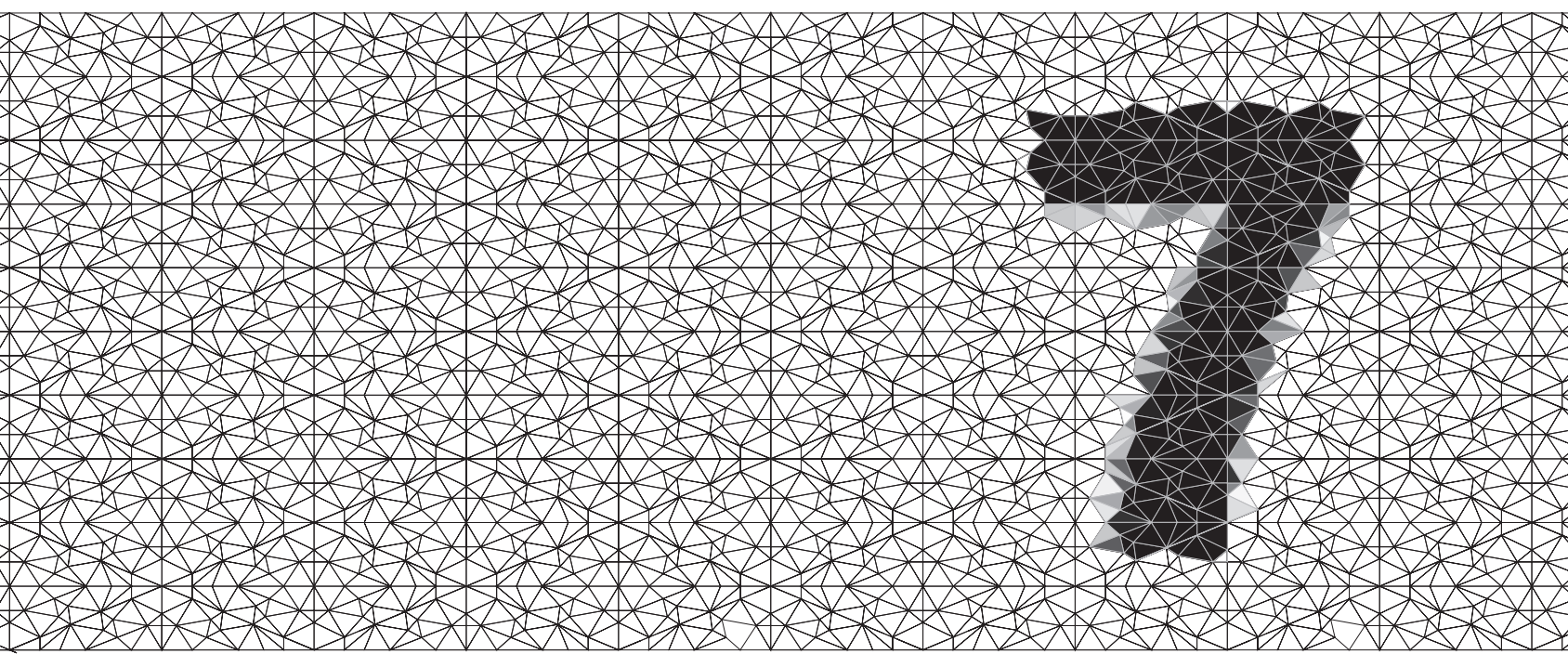

\section{Quantitative analysis of amplitude-integrated electroencephalogram patterns in stable preterm infants, with normal neurological development at one year}

Hendrik J. Niemarkt Peter Andriessen Chris H.L. Peters Jaco W. Pasman Carlos E. Blanco

Luc J. Zimmermann Sidarto Bambang Oetomo 


\section{Abstract}

\section{Background}

The amplitude-integrated EEG (aEEG) is feasible for monitoring cerebral activity in preterm infants. However, quantitative data on normal patterns in these infants are limited.

\section{Objective}

To study maturational aEEG changes in a cohort of stable preterm infants by automated quantification.

\section{Methods}

In a cohort of stable preterm infants with gestational age $(G A)<32$ weeks and normal neurological follow-up at 1 year, weekly $4 \mathrm{~h}$ EEG recordings were performed. aEEG traces were obtained from channel $C_{3}-C_{4}$. The upper margin amplitude (UMA), lower margin amplitude (LMA) and bandwidth (BW) were quantitatively calculated using an expert software system. In addition, the relative duration of discontinuous background pattern (discontinuous background defined as activity with $L M A<5 \mu \mathrm{V}$, expressed as DC-\%) was calculated.

\section{Results}

79 aEEG recordings (4-6 recordings/infant) were obtained in 18 infants. Analysis of the first week recordings demonstrated a strong positive correlation between GA and LMA, while DC-\% decreased significantly. Longitudinally, all infants showed increase of LMA. Multivariate analysis showed that GA and postnatal age (PA) both contributed independently and equally to LMA and DC-\%. We found a strong correlation between postmenstrual age $(G A+P A)$ and LMA and DC-\%, respectively.

\section{Conclusion}

To our knowledge, this is the first study were aEEG development was studied by automated quantification of aEEG characteristics in a cohort of stable preterm infants with a normal neurological development at 1 year of age.LMA and DC-\% are simple quantitative measures of neurophysiologic development and may be used to evaluate neurodevelopment in infants. 


\section{Introduction}

In the neonatal intensive care unit (NICU), amplitude-integrated electroencephalography (aEEG) is mainly used for the evaluation of brain function and the detection of convulsions in full-term hypoxic-encephalopatic infants[1]. The aEEG background patterns have proven to be of prognostic value in these infants [2].

The aEEG is also feasible for monitoring cerebral activity in preterm infants during intensive care management $[3,4]$. Compared to aEEG studies in asphyxiated term babies, knowledge concerning normal aEEG activity in preterm infants is still limited. Furthermore, normal aEEG background patterns in preterm infants are more diverse and related to gestational age (GA) and mature with increasing postmenstrual age (PMA). Changes in amplitude of the tracing's lower margin, bandwidth, and continuity (defined as electrical variation in the tracing)and the presence or absence of sleep-wake cycling have been used to describe aEEG maturation [5,6]. However, as most of the standard equipment presents aEEG data only graphically, the background pattern, lower margin amplitude (LMA), upper margin amplitude(UMA) and bandwidth (BW) of the aEEG have been determined by visual inspection of the tracing only. For example, earlier studies estimated LMA and BW by drawing horizontal lines through the tenth highest and tenth lowest amplitudes points in 2-min epochs $[7,8]$ or by drawing 'weighed lines' through the upper and lower edges of the recordings [9]. Both methods result in a rough estimation of the LMA and BW. Recently, new digital (a)EEG recording devices present the aEEG not only graphically but also provide quantitative data of several aEEG characteristics.

Before using aEEG on a large scale in extremely low birth weight infants, normal patterns and reference values of LMA, UMA and BW need to be established. Therefore, our objective was to assess changes of aEEG activity prospectively in healthy preterm infants with a $G A<32$ weeks, by analyzing quantitatively LMA, UMA, BW and duration of discontinuous pattern of (DC-\%) the aEEG.

\section{Methods}

\section{Study Subjects}

The aEEG study was part of a 24-channel neonatal EEG study and conducted in the NICU of Máxima Medical Centre in Veldhoven, The Netherlands during the period from May 2006 to October 2007. The hospital's ethics committee approved the study. Infants were enrolled in the study after written informed consent from the parents.

During the study period, 449 infants were admitted to our NICU (figure 1). Of these, 95 infants were eligible for the study, based on 'first-day' inclusion criteria: preterm infants with a $\mathrm{GA}<32$ weeks, birth weight appropriate for GA, Apgar score $\geq 6$ at $5 \mathrm{~min}$ and arterial umbilical pH $>7.00$, normal cerebral ultrasonography (intraventricular hemorrhage grade $\leq \mathrm{I}$ ) [10] and infants who were expected to stay longer than 2 weeks in our hospital.

Of the eligible population, 67 subjects were subsequently excluded in the first week because of cardiovascular instability, requiring volume expansion and/or inotropic medication, abnormalities on cranial ultrasound (intraventricular hemorrhage II-IV and all grades of periventricular leucomalacia 
[11]), use of any sedative or anti-epileptic medication, subjects with recurrent apnea treated with doxapram, or no parental consent.

Thus, 28 infants enrolled in the study. Of these, 10 subjects were excluded from analysis because they met exclusion criteria after the first week $(n=6)$, developed chronic lung disease $(n=2$, defined as the need for supplemental oxygen/ventilation at 36weeks' PMA [12]) or showed mental or motor Bayley Infant Developmental Scores $<85$ at 12 months of age $(n=2)$. Finally, 18 clinically stable preterm infants with at least 4 EEG recordings and a normal neurological follow-up at 12 months of age were analyzed in this paper.

The characteristics of these subjects are shown in table 1. Cranial ultrasonography was initially performed in all preterm infants in the first 3 days of life. Thereafter, studies were performed weekly until 1 month of age and repeated at term. Two of the subjects showed signs of germinal

Figure 1: Overview of inclusion and exclusion. Eighteen clinically stable preterm infants with a normal neurological follow-up at 12 month were included.

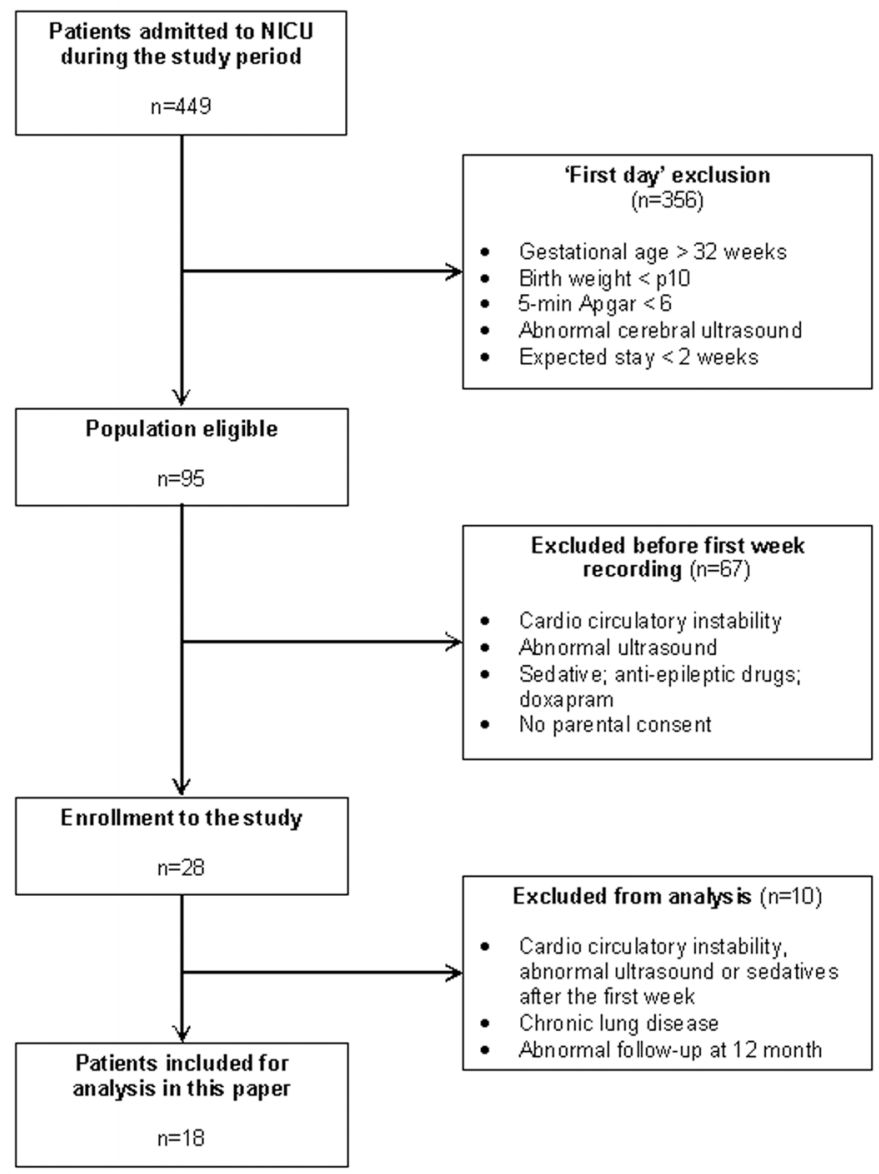


Table 1: Title: Characteristics of study population $(n=18)$.

\section{Clinical characteristics}

\begin{tabular}{ll}
\hline Gestational age (wk) & $29.0 \pm 0,3$ \\
\hline Birth weight (g) & $1297 \pm 58$ \\
\hline Female rate (\%) & 39 \\
\hline 5-min Apgar score & $8.5 \pm 1.3$ \\
\hline Umbilical arterial pH & $7.3 \pm 0.1$ \\
\hline Tocolysis (\%) & 83 \\
\hline Antenatal corticoids (\%) & 94 \\
\hline Caesarean section (\%) & 28 \\
\hline CRIB score (median and range) & $1(0-2)$ \\
\hline Mental Bayley score at 12-m & $104 \pm 12$ \\
\hline Motor Bayley score at 12-m & $95 \pm 10$ \\
\hline Legend: CRIB, clinical risk index for babies; values expressed as mean \pm SD, percentage or median (range). \\
\hline \hline
\end{tabular}

Table 2: Correlation between gestational age and quantitative aEEG parameters.

\begin{tabular}{|c|c|c|}
\hline & Pearson correlation coefficient & p-value \\
\hline LMA & +0.66 & $\mathrm{p}<0.01$ \\
\hline UMA & -0.02 & ns \\
\hline BW & -0.13 & ns \\
\hline DC-\% & -0.71 & $p<0.01$ \\
\hline
\end{tabular}

Legend: Data from 18 preterm infants with recordings performed in the first week of life. Lower margin amplitude (LMA), upper margin amplitude (UMA); bandwith (BW); relative duration discontinuous background, defined as activity with LMA < $5 \mu \mathrm{V}$ and expressed as DC-\%.

matrix hemorrhage, in 2 subjects transient flaring was observed only in the first week. All subjects showed normal indexes for the ventricles. All infants received caffeine at midnight with serum levels within therapeutic range. The clinical risk index for babies (CRIB score) was used to determine illness severity in the study population [13]. Infants for the study were followed in our centre at 6 and 12 months of corrected age, using the Bayley Scales of Infant Development for mental and motor function at 12 months of age.

\section{Data Acquisition and Analysis}

Starting at the end of the first week of life, weekly 24-channeldigital EEG recordings (NicoletOne; Viasys Healthcare, Conshohocken,Pa., USA) were performed as part of a more comprehensive neonatal EEG study. Each recording was classified into the following age terminology during the perinatal period: GA, postnatal age (PA), and postmenstrual age (PMA) (obtained by adding GA 
and PA). After skin preparation (Nuprep Gel; D.O. Weaver Co., Aurora, Colo., USA) Ag/AgCl cup electrodes, filled with a conductive paste (Ten2o; D.O. Weaver Co.), were placed according to the international 10-20 reduced montage system [14]. The impedance of the signal was $<10 \mathrm{k} \Omega$. The raw aEEG signal of channel $\mathrm{C}_{3}-\mathrm{C}_{4}$ (central region) was processed by filtering, rectifying and smoothing as described by Rosén [15]. Thereafter, the resulting aEEG signal was analyzed.

All recordings took place from 8 to 12 a.m. During the 4-hourrecording the infants were in prone or side position. Feeding and care for the infant were carried out according to the normal routine of the NICU.

The NicoletOne monitor produces quantitative output of LMA and UMA values. This data output is available as a text file with a time resolution of $1 \mathrm{~s}$. To obtain amplitude values which are in accordance with standard clinical aEEG traces (time resolution of $15 \mathrm{~s}$ ), resampling of data was performed to $15 \mathrm{~s}$. Extreme UMA values $(>100 \mu \mathrm{V})$, which were assumed to be related to artifacts using the recording (touching of the electrodes, movement), were removed. Thus, less than $5 \%$ of the data values were removed.

From the resampled aEEG, the following parameters were calculated: LMA, UMA and BW (BW=UMA-LMA). Continuous (C) tracings were defined as tracings where the LMA was $>5 \mu \mathrm{V}$ and discontinuous $(D C)$ tracings as tracings where the LMA was $<5 \mu \mathrm{V}$ [16]. Discontinuity of the aEEG tracings was quantitatively determined by calculating the time percentage during which the LMA was $<5 \mu \mathrm{V}$ and expressed as DC-\%.

\section{Statistical Analysis}

Data were statistically analyzed with SPSS 13.1 (SPSS; Inc., Chicago, III., USA). The Pearson correlation coefficient $r$ was used to evaluate the correlation between age parameters (GA, PA and PMA) and aEEG parameters (LMA, UMA, BW). In addition, to compare the influence of GA and PA on the quantitative aEEG parameters, a multivariate analysis was used. Multivariate and linear regression analysis results are shown wit $R^{2}$ and $\beta$ coefficients with $95 \%$ confidence intervals. A p value $<0.05$ was considered statistically significant.

\section{$\underline{\text { Results }}$}

Eighteen clinically stable infants (GA $29.0 \pm 0.3$ weeks; birth weight 1,297 \pm 58 g; median CRIB score 1) were studied and underwent $4-6$ weekly aEEG recordings. All aEEG recordings $(n=79)$ represented a large variability in PMA, ranging from 28 to 36 weeks. See table 1 for details of the population.

All recordings showed a continuous (C) or discontinuous (DC) background pattern. In all recordings, signs of periodic BW changes were observed suggestive for an alternating sleep and awake state [17-19]. None of the (a)EEG recordings showed evidence of neonatal seizure.

Eighteen aEEG recordings, performed in the first week of life, were studied to evaluate the effect of GA on the quantitative aEEG parameters. With advancing GA, LMA increased and DC-\% decreased. Table 2 shows the Pearson correlation coefficients between GA and quantitative aEEG parameters (UMA, LMA, BW, and DC-\%). In contrast, GA was not correlated with UMA and BW.

Postnatal changes in aEEG parameters were studied using all EEG recordings. In all infants, 
LMA increased with PA. In all but one, DC-\% decreased with PA. To discriminate whether GA or PA influences aEEG development, a multivariate model with quantitative aEEG parameters as dependent variable was applied. Multivariate analysis demonstrated that GA and PA contributed independently to LMA (LMA $=-6.7+0.35 \times \mathrm{GA}+0.39 \times \mathrm{PA} \mu \mathrm{V} ; \mathrm{p}<0.001)$ and $\mathrm{DC}-\mathrm{\%}(\mathrm{DC}-\%=345.8-9.0$ $\times$ GA-9.7 $\times$ PA \%; $p<0.001$ ). GA and PA accounted for 58 and $65 \%$ of the observed variation in LMA and $D C-\%$, respectively. Multivariate analysis showed a small but significant contribution of GA and PA on BW, accounting for less than $20 \%$ of the estimated variance in BW.

Figure 2:
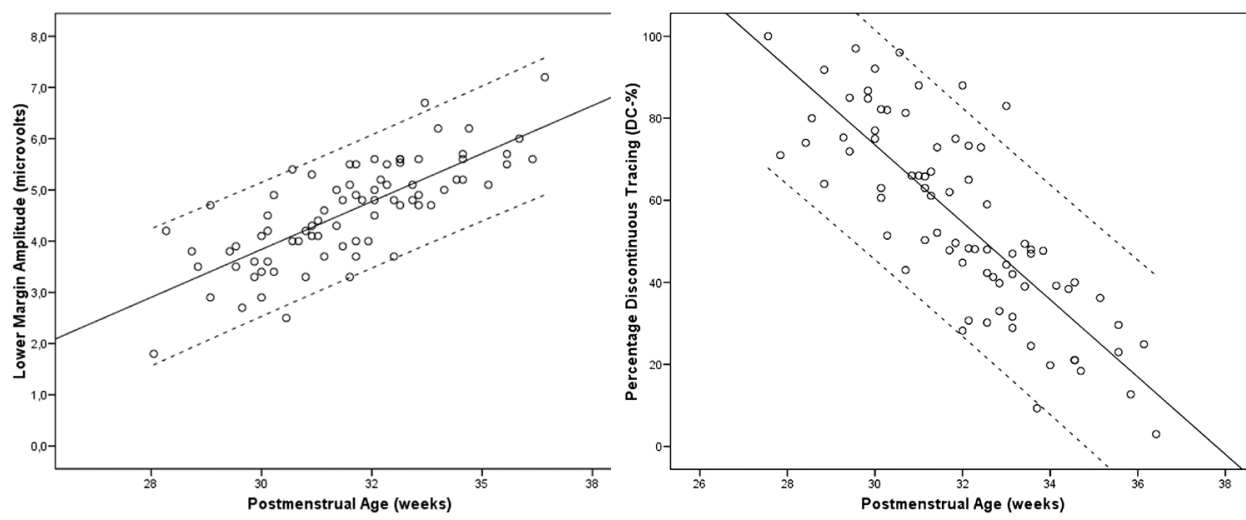

Legend: All seventy-nine recordings were used to study the relation between postmenstrual age (gestational age + postnatal age) and lower margin amplitude and the relative duration of discontinuous pattern DC-\%. Lower margin amplitude (correlation coefficient $r+0,76, p<0.001$ ) and relative duration of discontinuous pattern DC-\% (correlation coefficient $r-0.81, p<0.001$ ) changes as function of postmenstrual age. The mean values with $95 \%$ confidence interval are depicted.

Although the contribution of PA on LMA and DC-\%was somewhat stronger than the contribution of $G A$, this was of no statistical significance. Therefore, the relation between quantitative aEEG characteristics and PMA (GA + PA) was investigated in the pooled data set. Linear regression analysis demonstrated a significant correlation between PMA and LMA ( $r=0.76 ; p<0.001$ ) (figure 2a) and a significant negative correlation between PMA and DC- $\%(r=-0.81 ; p<0.001)$ (figure $2 b)$.

\section{Discussion}

This is the first study where maturational aEEG aspects are studied by automated quantification in a cohort of clinically stable preterm infants and normal neurologicalfollow-up at 1-year. We observed a positive correlation between LMA and age parameters GA, PA or PMA.DC-\% is negatively correlated with age parameters GA, PA or PMA. 


\section{LMA and DC-\% as Quantitative Parameters}

The positive correlation between LMA and age parameters is in agreement with other authors who found an increase in LMA with PMA. Sisman et al. [6] studied the LMA during high and low activity periods, respectively. These investigators observed an increase in LMA during low activity periods from approximately $3 \mu \mathrm{V}$ at PMA 25-28 weeks to approximately $5 \mu \mathrm{V}$ at PMA 33-35 weeks. This observation is comparable with our results. In this study, LMA was visually determined during high activity periods (active sleep) and during low activity periods (quiet sleep). However, how these high and low activity periods were defined and identified is not clear from the paper. Therefore the reproducibility of this technique is limited. In addition, we assume that a mean LMA value taken over the whole recording represents the cerebral activity more accurately.

Burdjalov [5] also found increase in LMA with PMA. However, the aEEG amplitude of lower border was incorporated in a scoring system but not exactly quantified in this study. Kuint [20] studied different aspects of aEEG maturation in preterm infants. They showed an increase in LMA with PMA from approximately $2 \mu \mathrm{V}$ in a group of infants with a PMA of 24-30 weeks to approximately $5 \mu \mathrm{V}$ in a group of infants with aPMA of 35-41 weeks. However, in this study, LMA was measured only during the high activity periods. In addition, the exact method of measuring LMA was not explained.

We observed a significant decrease of EEG discontinuity with PMA. Connell et al. [21] investigated the amount of discontinuous tracing in first week raw EEG recordings of preterm infants. These investigators found a decrease in discontinuous activity from $27 \%$ at 28 weeks GA to $10 \%$ at 38 weeks. Eyre et al. [22] also investigated first week EEG recordings and found a decrease in discontinuity from $35 \%$ at 28 weeks GA to $10 \%$ at 36 weeks. However, both authors used different definitions of discontinuous EEG activity and definitions were not quantitative, making this technique difficult to reproduce. In 2 studies by the group of Olischar et al. [23] and Klebermass et al. [24] discontinuity was assessed in aEEG tracings of preterm infants. They visually distinguished 2 types of discontinuous tracing in 10-min epochs. The median duration of discontinuous low-voltage tracing (LMA $<3$ $\mu \mathrm{V}$ and UMA between 15 and $30 \mu \mathrm{V}$ ) decreased with GA from approximately $55 \%$ at 24 weeks to approximately $7 \%$ at $28-29$ weeks. The duration of the discontinuous high voltage tracing (LMA 3-5 $\mu \mathrm{V}$ and UMA 20-40 $\mu \mathrm{V}$ ) did not change significantly.

Recently, different authors used automated techniques to analyze aEEG and EEG in preterm infants. West et al. [25] studied different aspects of the EEG of preterm infants during the first days of life and found that continuity and median amplitude increased over the first week.

As the recordings were only performed during the first week, we speculate that the changes found in this study might be related with the early neonatal transitory phase and adaptation to the extra-uterine environment, rather than postnatal maturation of the brain. To control for the effects of this early extra-uterine adaptation, we started with recording at the end of the first week of life.

Wikstrom et al. [26] investigated aEEG recordings of very preterm infants performed at the first days of life. They demonstrated that minimum and maximum aEEG amplitudes are correlated with neonatal brain injury. However, the calculated minimum aEEG amplitudes did not correspond with the LMA of standard aEEG tracings. This might be caused by the fact that the data were not sampled with a resolution of $1 \mathrm{~s}$ while the standard aEEG recorders provide data with a resolution of $15 \mathrm{~s}$. 


\section{Electroencephalography and aEEG}

The change in LMA and DC-\% reflects the maturation of the conventional EEG in the preterm infant. In these infants, the EEG background pattern changes gradually from discontinuous trace, which consists of periods of EEG activity (burst) alternated with periods of EEG quiescence(inter-bursts), to continuous trace, consisting of periods with continuous EEG activity [27, 28]. This gradual change is characterized by shortening in relative duration of the discontinuous periods and - within the discontinuous periods - by shortening of inter-burst lengths and increase in burst lengths.

As LMA represents the minimum EEG activity during15 $s$ (after filtering, and smoothing and rectifying), the increase in LMA represents the decrease in inter-burst length, the increase in burst length or higher EEG activity during inter-burst periods [29]. Thus, the increase in LMA is related to an increase in EEG continuity. Indeed, in the aEEG classification system of Hellström-Westas et al. [16], the continuous and discontinuous background patterns in term infants are distinguished by LMA ( $<5$ and $>5 \mu \mathrm{V}$,respectively). As these background patterns are commonly used in the clinical setting, we also quantified the time percentage of discontinuous tracing (DC-\%). As expected, the correlation between LMA and DC-\%is very high.

The exact etiology of changes in EEG and aEEG continuity remains to be elucidated. It is suggested that the shortening of inter-burst and increase in burst length are related to the maturation of excitatory synapses and/or the loss of presumed subcortical systems responsible for the abrupt inhibition of cortical activity [30,31]. Others suggested that changes in EEG continuity are related to cortical folding in the preterm brain [32]. However, in a translational study by Tucker et al. [33], a similar increase in LMA was found in rat pups, whose brain is not gyrated.

As changes in EEG continuity in preterm infants are related to neurosonographic and clinical abnormalities and outcome $[34,35]$, aEEG continuity is an interesting subject to study.

\section{Maturational Aspects}

To investigate the contribution of GA and PA, we performed a multivariate analysis. We observed that GA and PA independently influenced most aEEG characteristics. However, there was no significant difference between the amount of influence of GA and PA on LMA. This is in contrast with a semi quantitative aEEG study by Klebermass et al. [24], who postulated that extra-uterine aEEG development in preterm infants was accelerated. Sisman et al. [6] found indications for accelerated aEEG developmentin more immature infants as well. This contrast may be explained by the fact that we did not include small-for-gestational-age infants. Scherjon et al. [36] described small-forgestational-age infants who had an accelerated neurophysiologic development. This was related to adverse neurocognitive outcome at 5 years. Accelerated brain development is suggested to be caused by an adaptation of the immature brain to non-physiologic environmental circumstances in the NICU $[24,37,38]$.

The individual regression lines showed that in all - except for 2- infants the LMA increases and DC-\% decreases with age. Detailed analysis of the medical records of these 2 infants did not reveal any particular pre-, peri- or postnatal circumstances.

As aEEG characteristics were not influenced differently by GA and PA, their relation with PMA was investigated. Indeed, a linear relation between PMA and LMA and DC- $\%$ was demonstrated. PMA explained $58 \%$ of the variance in LMA and $65 \%$ of the variance in DC- $\%$. At visual inspection, 
we observed periodic variations in BW even in very preterm infants. This may be a sign of sleep wake cycling $[17,19,39]$. We did not try to quantify the periodic variations in BW. However, this may be of interest for a subsequent study.

\section{Limitations}

First, the aim of this study was to collect EEG data in a cohort of preterm infants without any additional pathologic conditions that might interfere with normal cerebral development. Therefore, we applied strict inclusion and exclusion criteria for the study, resulting in a relatively small population. However, all infants included in the study were normal at 1 year of follow-up. Second, the investigated aEEG characteristics were derived from the (central) $C_{3}-C_{4}$ channel and not the conventional parietal $\mathrm{P}_{3}-\mathrm{P}_{4}$ channel. This may lead to differences in aEEG tracings. However, the parietal and central brain regions are anatomically and functionally closely related to each other. Third, the recordings with $4 \mathrm{~h}$ duration were relatively short compared to the long-term application of aEEG in the NICU. However, all recordings were performed at the same time of day. Therefore, we think that potential diurnal variation between recordings was minimized. Also, the recordings incorporated more than 3 sleep cycles, thereby minimizing confounding effects of ultradian cyclicity on the recordings.

\section{Conclusion}

We analyzed different aEEG parameters by automatic quantification in relation to maturation in a cohort of clinically stable preterm infants with normal neurological follow-up at 1 year. LMA and DC-\% showed a strong correlation with PMA. LMA and DC-\% are simple quantitative markers of neurophysiologic development and may be used to assess neurodevelopment in infants. It is interesting to investigate if infants with perinatal complications and abnormal follow-up show a different LMA or DC-\% change with PMA or show values outside the $95 \%$ confidence interval. In this way quantitative aEEG analysis may become of value in predicting neurological outcome.

\section{Acknowledgments}

We would like to thank Ad Smets, Department of Clinical Neurophysiology of the Máxima Medical Centre, for his advice and assistance on performing the EEG recordings. We also would like to thank T. Katgert, Department of Psychology of the Máxima Medical Centre, for performing the follow-up examinations in the studied infants. 


\section{References}

1. de Vries LS, Hellstrom-Westas L. Role of cerebral function monitoring in the newborn. Arch Dis Child Fetal Neonatal Ed 2005;90(3):F201-7.

2. de Vries LS, Toet MC. Amplitude integrated electroencephalography in the full-term newborn. Clin Perinatol 2006;33(3):619-32, vi.

3. Hellstrom-Westas L, Klette H, ThorngrenJerneck K, Rosen I. Early prediction of outcome with aEEG in preterm infants with large intraventricular hemorrhages. Neuropediatrics 2001;32(6):319-24.

4. Olischar M, Klebermass K, Kuhle S, Hulek M, Messerschmidt A, Weninger M. Progressive posthemorrhagic hydrocephalus leads to changes of amplitude-integrated EEG activity in preterm infants. Childs Nerv Syst 2004;20(1):41-5.

5. Burdjalov VF, Baumgart S, Spitzer AR. Cerebral function monitoring: a new scoring system for the evaluation of brain maturation in neonates. Pediatrics 2003;112(4):855-61.

6. Sisman J, Campbell DE, Brion LP. Amplitudeintegrated EEG in preterm infants: maturation of background pattern and amplitude voltage with postmenstrual age and gestational age. J Perinatol 2005;25(6):391-6.

7. Verma UL, Archbald F, Tejani NA, Handwerker $S M$. Cerebral function monitor in the neonate. I: Normal patterns. Dev Med Child Neurol 1984;26(2):154-61.

8. Viniker DA, Maynard DE, Scott DF. Cerebral function monitor studies in neonates. Clin Electroencephalogr 1984;15(4):185-92.

9. Thornberg E, Thiringer K. Normal pattern of the cerebral function monitor trace in term and preterm neonates. Acta Paediatr Scand 1990;79(1):20-5.

10. Volpe JJ. Neurology of the newborn. 5th Edition. Philadelphia: Elsevier; 2008.

11. de Vries $L S$, Eken $P$, Dubowitz $L M$. The spectrum of leukomalacia using cranial ultrasound.
Behav Brain Res 1992;49(1):1-6.

12. Lal MK, Manktelow BN, Draper ES, Field DJ. Chronic lung disease of prematurity and intrauterine growth retardation: a populationbased study. Pediatrics 2003;111(3):483-7.

13. The CRIB (clinical risk index for babies) score: $a$ tool for assessing initial neonatal risk and comparing performance of neonatal intensive care units. The International Neonatal Network. Lancet 1993;342(8865):193-8.

14. Tekgul H, Bourgeois BF, Gauvreau K, Bergin AM. Electroencephalography in neonatal seizures: comparison of a reduced and a full 10/20 montage. Pediatr Neurol 2005;32(3):155-61.

15. Rosen I. The physiological basis for continuous electroencephalogram monitoring in the neonate. Clin Perinatol 2006;33(3):593-611, v.

16. Hellstrom-Westas L, Rosen I, De Vries LS, Greisen G. Amplitude-integrated EEG classification and interpretation in preterm and term infants. Neoreviews 2006;7(2):e76-e86.

17. Kuhle S, Klebermass K, Olischar M, Hulek M, Prusa AR, Kohlhauser C, et al. Sleep-wake cycles in preterm infants below 30 weeks of gestational age. Preliminary results of a prospective amplitude-integrated EEG study. Wien Klin Wochenschr 2001;113(7-8):219-23.

18. Olischar M, Klebermass $K$, Waldhoer T, Pollak A, Weninger M. Background patterns and sleep-wake cycles on amplitude-integrated electroencephalography in preterms younger than 30 weeks gestational age with peri-/ intraventricular haemorrhage. Acta Paediatr 2007;96(12):1743-50.

19. Scher MS, Johnson MW, Holditch-Davis D. Cyclicity of neonatal sleep behaviors at 25 to 30 weeks' postconceptional age. Pediatr Res 2005;57(6):879-82.

20. Kuint J, Turgeman A, Torjman A, Maayan-Metzger A. Characteristics of amplitude-integrated 
electroencephalogram in premature infants. J Child Neurol 2007;22(3):277-81.

21. Connell JA, Oozeer R, Dubowitz V. Continuous 4-channel EEG monitoring: a guide to interpretation, with normal values, in preterm infants. Neuropediatrics 1987;18(3):138-45.

22. Eyre JA, Nanei S, Wilkinson AR. Quantification of changes in normal neonatal EEGs with gestation from continuous five-day recordings Dev Med Child Neurol 1988;30(5):599-607.

23. Olischar M, Klebermass K, Kuhle S, Hulek $M$, Kohlhauser C, Rucklinger E, et al. Reference values for amplitude-integrated electroencephalographic activity in preterm infants younger than 30 weeks' gestational age. Pediatrics 2004;113(1 Pt 1):e61-6.

24. Klebermass K, Kuhle S, Olischar M, Rucklinger E, Pollak A, Weninger M. Intra- and extrauterine maturation of amplitude-integrated electroencephalographic activity in preterm infants younger than 30 weeks of gestation. Biol Neonate 2006;89(2):120-5.

25. West CR, Harding JE, Williams CE, Gunning MI, Battin MR. Quantitative electroencephalographic patterns in normal preterm infants over the first week after birth. Early Hum Dev 2006;82(1):43-51.

26. Wikstrom S, Ley D, Hansen-Pupp I, Rosen I, Hellstrom-Westas L. Early amplitude-integrated EEG correlates with cord TNF-alpha and brain injury in very preterm infants. Acta Paediatr 2008;97(7):915-9.

27. Holmes GL, Lombroso CT. Prognostic value of background patterns in the neonatal EEG. $J$ Clin Neurophysiol 1993;10(3):323-52.

28. Selton D, Andre M, Hascoet JM. Normal EEG in very premature infants: reference criteria. Clin Neurophysiol 2000;111(12):2116-24.

29. Hayakawa M, Okumura A, Hayakawa F, Watanabe K, Ohshiro M, Kato $Y$, et al. Background electroencephalographic (EEG) activities of very preterm infants born at less than 27 weeks gestation: a study on the degree of continuity. Arch Dis Child Fetal Neonatal Ed 2001;84(3):F163-7.

30. Vecchierini MF, d'Allest $A M$, Verpillat P. EEG patterns in 10 extreme premature neonates with normal neurological outcome: qualitative and quantitative data. Brain Dev 2003;25(5):330-7.

31. Victor S, Appleton RE, Beirne M, Marson AG, Weindling AM. Spectral analysis of electroencephalography in premature newborn infants: normal ranges. Pediatr Res 2005;57(3):336-41.

32. Biagioni E, Frisone MF, Laroche S, Kapetanakis BA, Ricci D, Adeyi-Obe M, et al. Maturation of cerebral electrical activity and development of cortical folding in young very preterm infants. Clin Neurophysiol 2007;118(1):53-9.

33. Tucker AM, Aquilina K, Chakkarapani E, Hobbs CE, Thoresen M. Development of amplitude-integrated electroencephalography and interburst interval in the rat. Pediatr Res 2009;65(1):62-6.

34. Benda Gl, Engel RC, Zhang YP. Prolonged inactive phases during the discontinuous pattern of prematurity in the electroencephalogram of very-low-birthweight infants. Electroencephalogr Clin Neurophysiol 1989;72(3):189-97.

35. Conde JR, de Hoyos AL, Martinez ED, Campo CG, Perez AM, Borges AA. Extrauterine life duration and ontogenic EEG parameters in preterm newborns with and without major ultrasound brain lesions. Clin Neurophysiol 2005;116(12):2796-809.

36. Scherjon S, Briet J, Oosting H, Kok J. The discrepancy between maturation of visual-evoked potentials and cognitive outcome at five years in very preterm infants with and without hemodynamic signs of fetal brain-sparing. Pediatrics 2000;105(2):385-91.

37. Gressens $P$, Rogido $M$, Paindaveine $B$, Sola A. The impact of neonatal intensive care practices on the developing brain. J Pediatr 2002;140(6):646-53.

38. Scher MS, Jones BL, Steppe DA, Cork DL, 
Seltman HJ, Banks DL. Functional brain maturation in neonates as measured by EEG-sleep analyses. Clin Neurophysiol 2003;114(5):875-82.

39. Curzi-Dascalova L, Figueroa JM, Eiselt $M$, Christova E, Virassamy A, d'Allest AM, et al. Sleep state organization in premature infants of less than 35 weeks' gestational age. Pediatr Res 1993;34(5):624-8. 


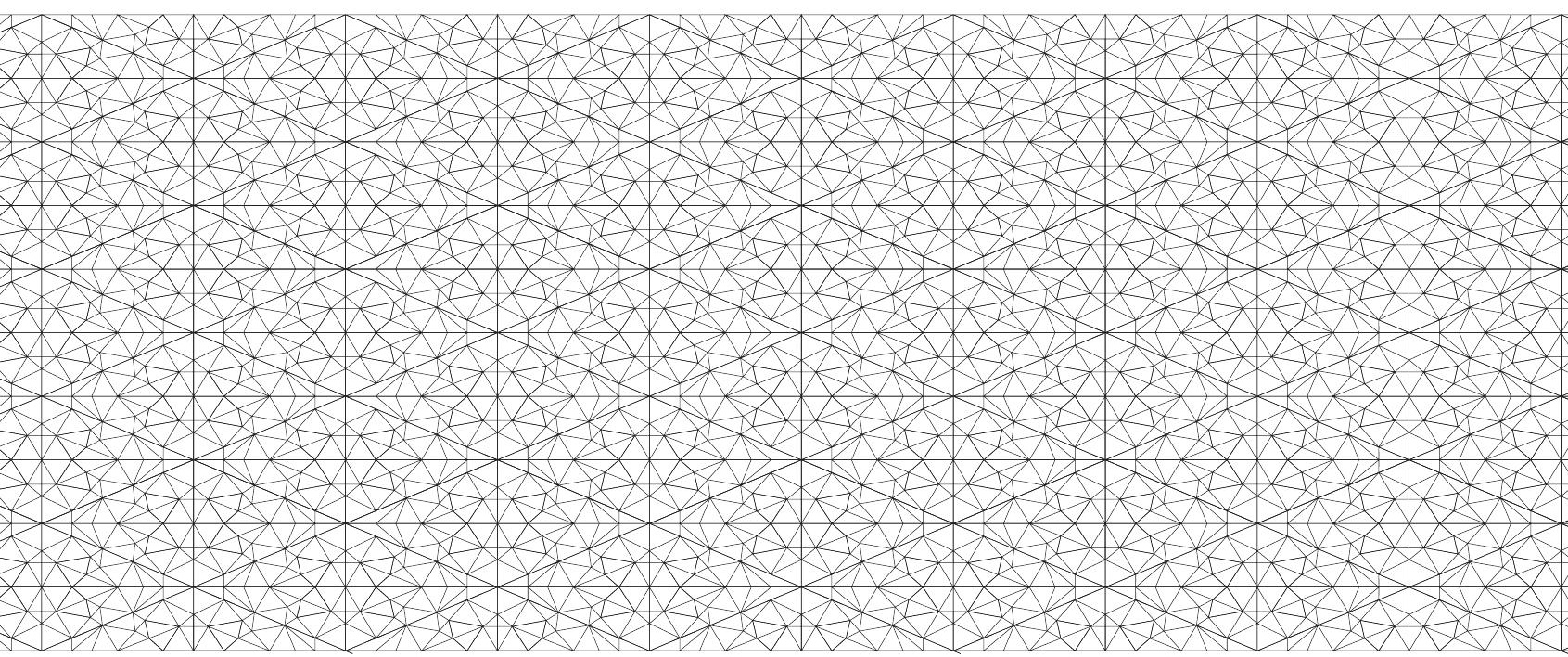




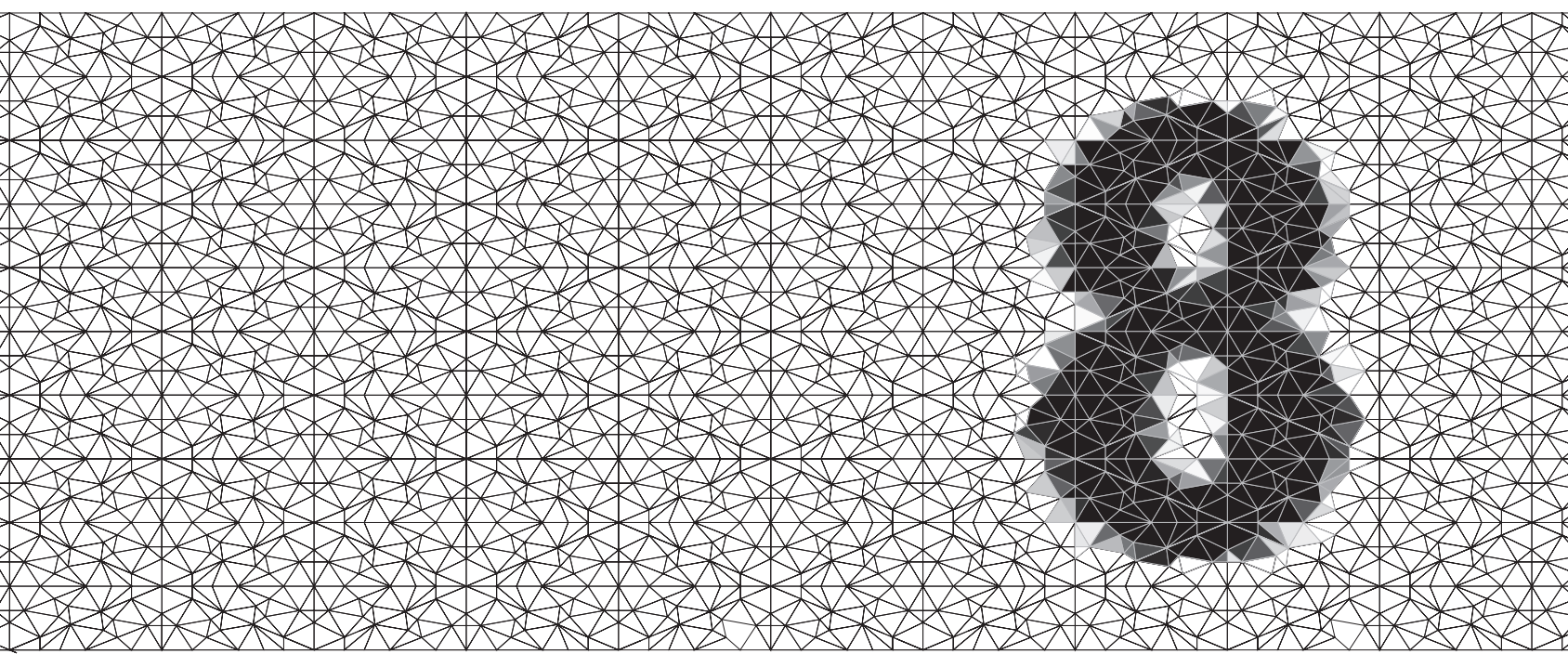

\section{Multi-channel amplitude-integrated EEG characteristics in preterm infants with a normal neurodevelopment at two years of corrected age}

Hendrik J. Niemarkt

Ward Jennekens Imke A. Maartens Tessa Wassenberg Marijke van Aken Titia Katgert Boris W. Kramer Antonio W.D. Gavilanes Luc J. Zimmermann Sidarto Bambang Oetomo Peter Andriessen 


\section{Abstract}

\section{Aim}

To analyze quantitatively multi-channel amplitude-integrated EEG (aEEG) characteristics and assess regional differences.

\section{Methods}

We investigated 40 preterm infants (postmenstrual age, PMA: range 27-37 weeks) with normal followup at 24 months of age, at a median postnatal age of 8 days using 4-h EEG recordings according to the international 10-20 system reduced montage. Nine ( 3 transverse and 6 longitudinal) channels were selected and converted to aEEG registrations. For each aEEG registration, lower margin amplitude (LMA), upper 32 margin amplitude (UMA) and bandwidth (UMA-LMA) were calculated.

\section{Results}

In all channels PMA and LMA showed strong positive correlations. Below 32 weeks of PMA, LMA was $\leq 5 \mu \mathrm{V}$. Linear regression analysis showed a maximum LMA difference between channels of approximately 2 and $1 \mu \mathrm{V}$ at 27 and 37 weeks of PMA, respectively. The lowest are LMA values in the occipital channel and the 36 highest values are in centro-occipital channels. In the frontal, centro-temporal and centro-occipital channels, UMA and bandwidth changed with PMA. No differences in LMA, UMA and bandwidth were found between hemispheres. Skewness of LMA values strongly correlated with PMA, positive skewness indicating an immature brain (PMA $\leq 32$ weeks) and negative skewness a maturing (PMA>32 weeks) brain.

\section{Conclusions}

We detected symmetric increase of aEEG characteristics, indicating symmetric brain maturation of the left and right hemispheres. Our findings demonstrate the clinical potential of computer-assisted analyses of aEEG recordings in detecting maturational features which are not readily identified visually. This may provide an objective and reproducible method for assessing brain maturation and long-term prognosis. 


\section{Introduction}

\section{Background}

The survival of preterm infants has increased due to improved care in the delivery room and neonatal intensive care unit [1]. However, it has become clear that there is an increased rate of neurodevelopmental abnormalities in preterm infants, even in the absence of ultrasonographic abnormalities $[2,3]$. Studying electrophysiological maturation in preterm infants may be a useful tool to detect neurodevelopmental delay $[4,5]$.

Conventional electroencephalography (EEG) is the gold standard in the assessment of electrophysiological cerebral function. A nine-electrode reduced $10-20$ montage system is a sensitive tool for identification of neonatal seizures and assessment of background characteristics of neonatal electroencephalography [6]. Typical neonatal EEG patterns (e.g. changes in continuity, specific waveforms, sleep-wake cycling) change as a function of gestational age (GA) as well as post-menstrual age (PMA, GA + postnatal age) [7]. Electrophysiological brain maturation in human newborns has been studied by several groups, mostly using multi-channel EEG recordings $[8,9]$.

Amplitude-integrated EEG (aEEG) is a technique in which the original signal of a single or two channel derivation is highly processed and expressed in a simplified manner, making it more easy to interpret to non-EEG experts [10]. Key features of the aEEG technique include signal amplification, band pass filtering, signal processing and display at a speed of $6 \mathrm{~cm} / \mathrm{h}$, ideal for long-term monitoring [11]. Single channel aEEG has shown to be a useful tool for continuous bedside monitoring of neonatal seizure and background patterns in asphyxiated full-term infants [12, 13]. The aEEG is also increasingly being used in preterm infants [14-17]. Several studies have provided normative data of aEEG characteristics (e.g. lower and upper margin amplitude; bandwidth) in preterm infants at different GA and PMA $[15,16]$. As with advancing PMA an increase of continuity is noted, lower and upper margin amplitude of the aEEG are related to PMA $[18,19]$. The lower margin amplitude of the aEEG increases with advancing PMA and is substantially lower than in term infants[15]. However, assessment of aEEG recordings is mainly based on visual interpretation of the aEEG pattern [20]. Today, digital EEG recorders provide the opportunity towards a computer-assisted signal analysis of EEG characteristics showing the aEEG traces simultaneously of several channels. Hence, the maturational change in lower and upper margin amplitude of the aEEG can be assessed quantitatively. In a previous study we showed the feasibility to study quantitatively single-channel aEEG characteristics and to correlate these with age [21]. For single channel aEEG, most advocate lead placement at the biparietal area ( $\left.\mathrm{P}_{3}-\mathrm{P}_{4}\right)$, which overlies a vascular watershed zone [10]. Using multi-channel aEEG, asymmetry in background was observed in full-term infantswith unilateral brain damage [22] and resulted in higher detection of subclinical seizures [23]. Although regional differences and interhemispheric asymmetry are observed in the raw preterm EEG [7], no study compared multi-channel aEEG tracings of the reduced 10-20 montage system using computerassisted signal analysis in preterm infants.

The aim of this study is to investigate if aEEG characteristics (e.g. lower and upper margin amplitude and bandwidth) show differences between brain regions and how these features mature with PMA. To evaluate this we performed multi-channel (reduced 10-20 montage) EEG recordings in stable preterm infants with a normal neurodevelopmental follow-up at 24 months of corrected age. 


\section{Materials and methods}

\section{Study subjects}

This study was part of a comprehensive EEG research program of Máxima Medical Center in Veldhoven, The Netherlands and conducted in the Department of Pediatrics. The hospital's ethics committee approved the study. Infants were enrolled in the study after written informed consent was obtained from the parents. Eligible for this study were appropriate-for-gestational age birth weight and clinically stable infants with a GA between 26 and 37 weeks. Preterm infants with a GAb32 weeks were recruited from the NICU between May 2006 and March 2009. Preterm infants with a GA between 32 and 37 weeks were recruited from the neonatal ward between September 2008 and March 2009.

During the study periods, 687 infants were admitted to the NICU $(n=630)$ or the neonatal ward $(n=57)$. Of these, 230 infants were eligible for the study, based on "first day" inclusion criteria: GA < 37 weeks, birth weight appropriate for GA (Np10), Apgar score $\geq 6$ at $5 \mathrm{~min}$ and arterial umbilical pH $>$ 7.0. Of the eligible population, 179 subjects were subsequently excluded in the first week because of cardiovascular instability requiring volume expansion and/or inotropic medication (hypotension, defined as mean blood pressure $<$ GA), abnormalities on cranial ultrasound (intraventricular hemorrhage $\geq$ grade II or periventricular leukomalacia), meningitis, use of any sedative, analgesics or anti-epileptic medication, subjects with recurrent apnea treated with doxapram, mortality or no parental consent.

Thus, 51 infants were enrolled in the study. Of these, 11 infants were excluded from analysis because they met exclusion criteria (see above, criteria) after the first week or showed developmental delay at 24 months of corrected age (see below, follow-up). Hence, 40 stable preterm infants with an uneventful clinical course ( 24 recruited from the NICU and 16 recruited from the neonatal ward) and a normal follow-up at 24 months of corrected age were analyzed in this paper. The characteristics of the study group are shown in Table 1.

Cranial ultrasonography was performed in all infants in the first week of life. In the subgroup $<32$ weeks gestation, cranial ultrasonography was performed weekly until 1 month of age and repeated at term. In 10 subjects of the study population, intraventricular hemorrhage grade I was observed. In 6 subjects of the study population, transient periventricular echodensities were detected with a duration of $<1$ week. The clinical risk index for babies was used to estimate illness severity of the study population [24].

\section{Follow-up of the study subjects}

The subgroup of very preterm infants with a GA $<32$ weeks $(n=24)$ was followed in our perinatal center at 6, 12 and 24 months of corrected age, using the Bayley Scales of Infant Development for mental and motor function at 12 and 24 months of corrected age. The subgroup of preterm infants with a $G A \geq 32(n=16)$ was considered to be a low-risk group. The low-risk group showed normal psychomotor development at the age of 3, 6, 12 and 24 months according to a standardized Dutch screening measure (VanWiechen Schedule), used at children health-care centers to monitor motor behavior, speech and communication skills [25]. 
Table 1: Demographics of the study population $(n=33)$.

\begin{tabular}{|c|c|}
\hline Gestational age (wk) & $30.4 \pm 2.9$ \\
\hline Postmenstrual age of study (wk) & $31.5 \pm 2.9$ \\
\hline Birth weight (g) & $1564 \pm 506$ \\
\hline 5-min Apgar score & $8.4 \pm 1.2$ \\
\hline Umbilical arterial pH & $7.27 \pm 0.08$ \\
\hline Caesarean section (\%) & $23 \%$ \\
\hline Female (\%) & $44 \%$ \\
\hline CRIB score (median and range) & $1(0-4)$ \\
\hline Mean pH & $7.32 \pm 0.07$ \\
\hline Mean Base Excess (mmol/l) & $-1.8 \pm 4.9$ \\
\hline Mean glucose (mmol/l) & $5.4 \pm 2.0$ \\
\hline MDI at 24 months & $106 \pm 12$ \\
\hline PDI at 24 months & $91 \pm 11$ \\
\hline \multicolumn{2}{|c|}{$\begin{array}{l}\text { CRIB: Clinical risk index for babies; values expressed as mean } \pm \text { SD, percentage or median (range). Mean pH, base excess and } \\
\text { mean glucose levels were calculated from } 300 \text { and } 359 \text { blood samples, respectively (obtained during the first week of life). } \\
\text { Mental developmental index (MDI) and psychomotor developmental index (PDI) was available for the } 22 \text { preterm infants } \\
\text { with a gestational age }<32 \text { weeks. }\end{array}$} \\
\hline
\end{tabular}

\section{Data acquisition}

The infants were investigated at a median postnatal age of 8 days (range, 6-11) using a multichannel EEG recording (NicoletOne ${ }^{\mathrm{TM}}$, Viasys Healthcare, Conshohocken, PA, USA). Each recording was classified into the following age terminology during the perinatal period: gestational age (GA), postmenstrual age (PMA) and postnatal age 166 (PA). After skin preparation (Nuprep Gel, D.O. Weaver, Aurora, CO, USA), Ag/AgCl cup electrodes, filled with a conductive paste (Ten2o, D.O.Weaver, Aurora, CO, USA), were placed on the head. The international reduced 10-20 montage system for neonates was used with combined transverse and longitudinal montage (figure 1) [6]. The inter-electrode distances of the transverse montage (frontal, Fp1- Fp2; occipital, O1-O2; centrotemporal left, $\mathrm{C}_{3}-\mathrm{T}_{3}$; centro-temporal right $\mathrm{C}_{4}-\mathrm{T}_{4}$ ) were equal to $20 \%$ of the diameter of the head. The inter-electrode distances of $\mathrm{C}_{3}-\mathrm{C}_{4}$ (central) and the longitudinal montage (centro-frontal left, $\mathrm{C}_{3}-\mathrm{Fp} 1$; centro-frontal right, $\mathrm{C}_{4}-\mathrm{Fp}_{2}$; centro-occipital left, $\mathrm{C}_{3}-\mathrm{O}_{1}$; centro-occipital right, $\mathrm{C}_{4}-\mathrm{O}_{2}$ ) were equal to $40 \%$ of the diameter of the head. Impedance value was verified during each recording and was $<10 \mathrm{k} \Omega$. All recordings took place between 8 and 12 am between rounds of nursing care. All subjects were in prone or side position. Feeding and care for the infant was carried out according the normal routine of the NICU or neonatal ward.

\section{Quantitative data analysis}

The entire raw EEG signal was converted into an aEEG signal using the clinical software system, provided by the NicoletOne ${ }^{\mathrm{TM}}$ device and exported as text files for off-line analysis. Extreme UMA 
values of $>300 \mu \mathrm{V}$, (which constituted b1\% of total data values), were assumed to be related to artifacts during the recording (touching of the electrodes, movement) and were therefore removed from analysis. The median length of the recordings was $221 \mathrm{~min}$ (range, 162-261) from which the following data were derived: 1 . lower margin amplitude (LMA): the lower edge of the tracing; 2. upper margin amplitude (UMA): the upper edge of the tracing; 3. bandwidth: difference between UMA and LMA.

Figure 1:

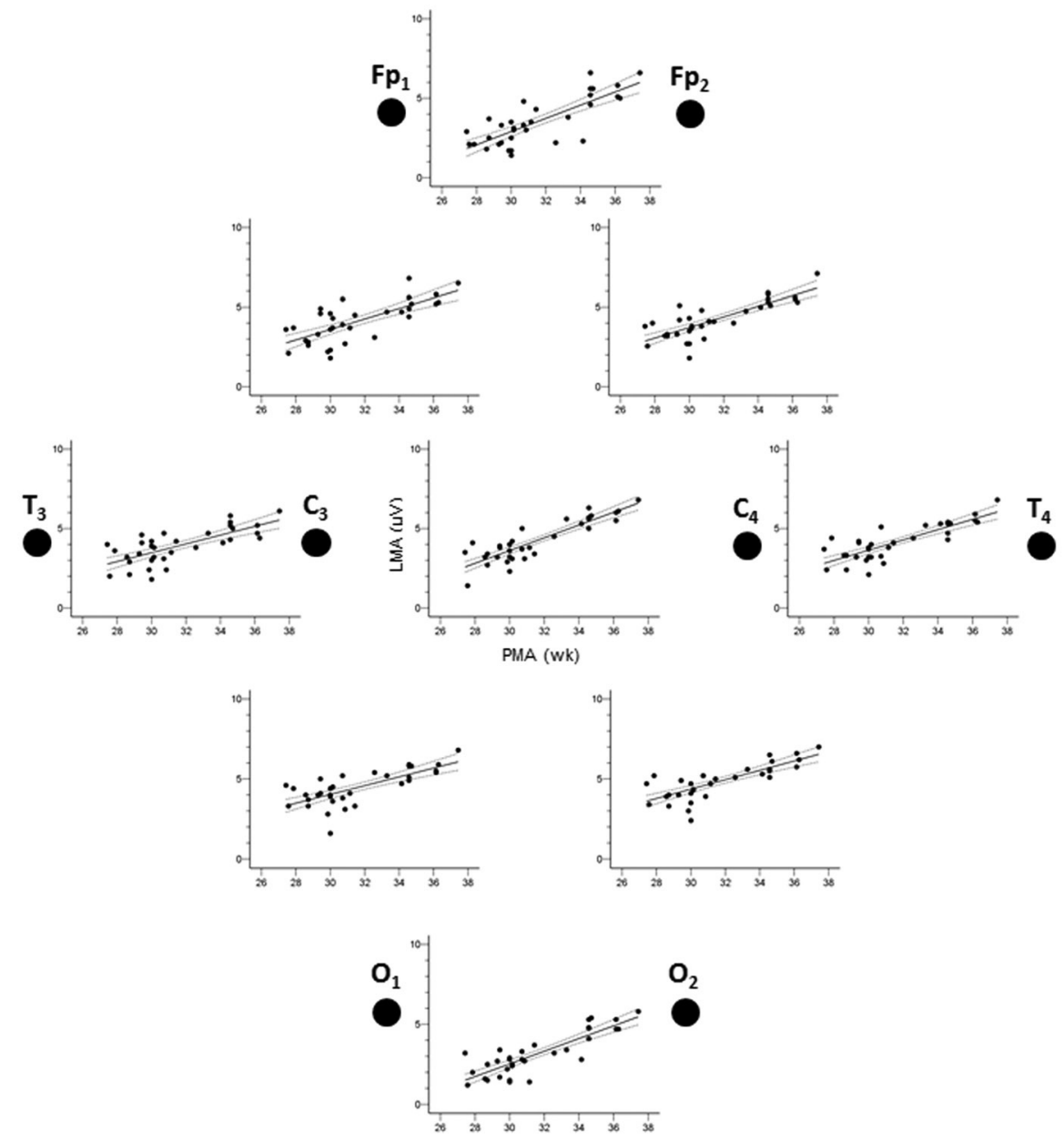

Figure 1 shows the regression lines and $95 \%$ confidence limits between postmenstrual age ( $x$-axis PMA, wk) and lower margin amplitude ( $y$-axis LMA, expressed in $\mu \mathrm{V}$ ) values for all channels. The inter-electrode distances of the transverse montage (frontal, $\mathrm{Fp}_{1}-\mathrm{Fp}_{2}$; occipital, $\mathrm{O}_{1}-\mathrm{O}_{2^{\prime}}$ c centro-temporal left, $\mathrm{C}_{3}-\mathrm{T}_{3^{\prime}}$ c centro-temporal right $\mathrm{C}_{4}-\mathrm{T}_{4^{\prime}}$ ) were equal to $20 \%$ of the diameter of the head. The inter-electrode distances of $\mathrm{C}_{3}-\mathrm{C}_{4}$ (central) and the longitudinal montage (centro-frontal left, $\mathrm{C}_{3}-\mathrm{Fp}_{1}$; centrofrontal right, $\mathrm{C}_{4}-\mathrm{Fp}_{2}$; centro-occipital left, $\mathrm{C}_{3}-\mathrm{O}_{1}$; centro-occipital right, $\mathrm{C}_{4}-\mathrm{O}_{2}$ ) were equal to $40 \%$ of the diameter of the head. The regression diagrams of the channels are plotted between the leads, indicated by the black dots. 
These aEEG characteristics were calculated for nine channels. Three aEEG channels (occipital channel $\mathrm{O}_{1}-\mathrm{O}_{2}$; central channel $\mathrm{C}_{3}-\mathrm{C}_{4}$; frontal channel Fp1-Fp2) measured the electrical activity over the occipital, central and frontal regions, respectively. In addition, hemispheric electrical activity was measured by three left (centro-occipital $\mathrm{C}_{3}-\mathrm{O}_{1}$; centro-temporal $\mathrm{C}_{3}-\mathrm{T}_{3}$; centro-frontal $\mathrm{C}_{3}-\mathrm{Fp}_{1}$ ) and three right hemispheric aEEG channels (centro-occipital $\mathrm{C}_{4}-\mathrm{O}_{2}$; centro-temporal $\mathrm{C}_{4}-\mathrm{T}_{4}$; centro-frontal $\mathrm{C}_{4}-\mathrm{Fp}_{2}$ ) (figure 1).

\section{Statistical analysis}

Statistical analysis was performed using SPSS for Windows version 13.0 (Inc, Chicago, IL, USA). Data with a normal distribution are expressed as a mean $\pm \mathrm{SD}$, otherwise data are expressed as a median and inter quartile range (IQR). As the amplitude values within the aEEG were not all normally distributed, median values of UMA, LMA and bandwidth were calculated for each subject. The calculated median values were normally distributed and used for linear regression analysis. The Pearson's correlation coefficient $r$ was used to evaluate the correlation between PMA and the aEEG characteristics (LMA, UMA and bandwidth). Linear regression analysis results are shown with $a$ - and b-coefficient (a, intercept; b, slope of regression line) and $95 \%$ confidence interval to study the influence of PMA on the quantitative aEEG characteristics.

Statistical significance was accepted with $p$-values less than 0.05 .

\section{Results}

We included 40 stable premature infants in this study, with a mean GA of 30.5 weeks (range 26-36 weeks) and mean PMA of 31.6 weeks (range 27-37 weeks) (Table 1). All aEEGs showed a variety of discontinuous and continuous background patterns without characteristic signs of neonatal seizures.

\section{Lower margin amplitude (Table 2)}

We observed strong positive correlations (varying between+0.70 and $+0.87 ; p<0.01$ ) between PMA and LMA for all nine channels. In general, below 32 weeks of PMA, LMA values were $\leq 5 \mu \mathrm{V}$ (figure 1). The lowest LMA values were found for the occipital channel, the highest values for the centrooccipital channels. The regression values calculated for 27 weeks of PMA indicated a difference of approximately $2 \mu \mathrm{V}$ between the occipital and centro-occipital channel. Likewise, at 27 weeks of PMA we found a difference of approximately $1 \mu \mathrm{V}$ between the occipital and central channel. The differences in LMA values became smaller at 37 weeks of PMA, with LMA values varying between approximately 5 to $6 \mu \mathrm{V}$.

The b-coefficient, indicating LMA change per week, varied between 0.3 and $0.4 \mu \mathrm{V} /$ week and was not different between the channels.

\section{Upper margin amplitude (Table 3)}

We observed strong positive correlations (varying between +0.54 and $+0.59 ; p<0.01$ ) between PMA and UMA for the frontal, centro-frontal and centro-occipital channels. The regression values calculated for 27 and 37 weeks of PMA were comparable between these chan-nels, with values 
Table 2: Linear regression analysis between PMA and lower margin amplitude

\begin{tabular}{|c|c|c|c|c|}
\hline & $\begin{array}{l}\text { Pearson } \\
\text { correlation }\end{array}$ & $\begin{array}{l}\text { Regression } \\
\text { value at } \mathbf{2 7} \text { wk } \\
\text { [95\% confidence } \\
\text { interval] }\end{array}$ & $\begin{array}{l}\text { Regression } \\
\text { value at } \mathbf{3 7} \mathbf{w k} \\
{[95 \% \text { confidence }} \\
\text { interval] }\end{array}$ & $\begin{array}{l}\text { b-coefficient } \\
\text { [95\% confidence } \\
\text { interval] }\end{array}$ \\
\hline Frontal (Fp1-Fp2) & $0.79\left(^{*}\right)$ & $1.7[1.0 ; 2.3]$ & $5.8[5.1 ; 6.5]$ & $0.42[0.30 ; 0.53]$ \\
\hline Central (C3-C4) & $0.88\left(^{*}\right)$ & $2.4[2.0 ; 2.8]$ & $6.4[5.9 ; 6.9]$ & $0.40[0.32 ; 0.48]$ \\
\hline Occipital (01-O2) & $0.85\left(^{*}\right)$ & $1.3[0.9 ; 1.8]$ & $5.3[4.7 ; 5.9]$ & $0.40[0.31 ; 0.49]$ \\
\hline Centro-frontal right (C4-Fp2) & $0.81\left(^{*}\right)$ & $2.7[2.3 ; 3.2]$ & $6.0[5.5 ; 6.6]$ & $0.33[0.24 ; 0.42]$ \\
\hline Centro-frontal left (C3-Fp1) & $0.73\left(^{*}\right)$ & $2.6[2.0 ; 3.2]$ & $5.9[5.2 ; 6.6]$ & $0.33[0.22 ; 0.44]$ \\
\hline Centro-temporal right (C4-T4) & $0.82\left(^{*}\right)$ & $2.7[2.2 ; 3.1]$ & $5.9[5.4 ; 6.4]$ & $0.32[0.24 ; 0.41]$ \\
\hline Centro-temporal left (C3-T3) & $0.72\left(^{*}\right)$ & $2.6[2.1 ; 3.2]$ & $5.4[4.8 ; 6.0]$ & $0.28[0.18 ; 0.37]$ \\
\hline Centro-occipital right (C4-O2) & $0.78\left(^{*}\right)$ & $3.5[3.0 ; 4.0]$ & $6.4[5.9 ; 6.9]$ & $0.29[0.21 ; 0.38]$ \\
\hline Centro-occipital left (C3-O1) & $0.72\left({ }^{*}\right)$ & $3.2[2.7 ; 3.7]$ & $5.9[5.4 ; 6.5]$ & $0.27[0.18 ; 0.37]$ \\
\hline \multicolumn{5}{|c|}{$\begin{array}{l}\text { Table } 2 \text { shows the Pearson correlation coefficient and linear regression analysis between postmenstrual age (PMA) and lower } \\
\text { margin amplitude (LMA) values of the aEEG for nine different channels. The regression values (LMA, } \mu \mathrm{V} \text { ) with } 95 \% \text { confidence } \\
\text { intervals are calculated for } 27 \text { and } 37 \text { weeks of gestation, respectively. The b-coefficient (slope) refers to the change of LMA } \\
\text { per week ( } \mu \mathrm{V} / \mathrm{wk} \text { ). } \\
\text { (*) P-value }<0.005 \text {. }\end{array}$} \\
\hline
\end{tabular}

Table 3: Linear regression analysis between PMA and upper margin amplitude

\begin{tabular}{|c|c|c|c|c|}
\hline & $\begin{array}{l}\text { Pearson } \\
\text { correlation }\end{array}$ & $\begin{array}{l}\text { Regression } \\
\text { value at } \mathbf{2 7} \mathbf{w k} \\
\text { [95\% confidence } \\
\text { interval] }\end{array}$ & $\begin{array}{l}\text { Regression } \\
\text { value at } \mathbf{3 7} \text { wk } \\
\text { [95\% confidence } \\
\text { interval] }\end{array}$ & $\begin{array}{l}\text { b-coefficient } \\
{[95 \% \text { confidence }} \\
\text { interval] }\end{array}$ \\
\hline Frontal (Fp1-Fp2) & $0.52(*)$ & $35[31 ; 39]$ & $23[19 ; 28]$ & $-1.2[-1.9 ;-0.5]$ \\
\hline Central (C3-C4) & 0.17 (NS) & $\mathrm{n} / \mathrm{a}$ & $\mathrm{n} / \mathrm{a}$ & $\mathrm{n} / \mathrm{a}$ \\
\hline Occipital (01-O2) & 0.04 (NS) & $\mathrm{n} / \mathrm{a}$ & $\mathrm{n} / \mathrm{a}$ & $\mathrm{n} / \mathrm{a}$ \\
\hline Centro-frontal right (C4-Fp2) & $0.59\left(^{*}\right)$ & $35[31 ; 39]$ & $21[16 ; 25]$ & $-1.5[-2.2 ;-0.7]$ \\
\hline Centro-frontal left (C3-Fp1) & $0.53\left(^{*}\right)$ & $32[28 ; 36]$ & $19[15 ; 24]$ & $-1.2[-2.0 ;-0.5]$ \\
\hline Centro-temporal right (C4-T4) & $0.55(*)$ & $43[37 ; 49]$ & $21[14 ; 28]$ & $-2.2[-3.4 ;-1.0]$ \\
\hline Centro-temporal left (C3-T3) & $0.57\left(^{*}\right)$ & $42[35 ; 48]$ & $19[11 ; 26]$ & $-2.3[-3.5 ;-1.1]$ \\
\hline Centro-occipital right (C4-O2) & 0.28 (NS) & $\mathrm{n} / \mathrm{a}$ & $\mathrm{n} / \mathrm{a}$ & $\mathrm{n} / \mathrm{a}$ \\
\hline Centro-occipital left (C3-O1) & 0.24 (NS) & $\mathrm{n} / \mathrm{a}$ & $\mathrm{n} / \mathrm{a}$ & $\mathrm{n} / \mathrm{a}$ \\
\hline
\end{tabular}

Table 3 shows the Pearson correlation coefficient and linear regression analysis between postmenstrual age (PMA) and upper margin amplitude (UMA) values of the aEEG for nine different channels. The regression values (UMA, $\mu \mathrm{V}$ ) with $95 \%$ confidence intervals are calculated for 27 and 37 weeks of gestation, respectively. The b-coefficient (slope) refers to the change of UMA per week ( $\mu \mathrm{V} / \mathrm{wk})$.

$\left(^{*}\right)$ P-value $<0.005 ;\left(^{* *}\right)$ P-value $<0.05$; NS refers to not significant; $n / a$ not applicable 
Table 4: Linear regression analysis between PMA and bandwidth

\begin{tabular}{|c|c|c|c|c|}
\hline & $\begin{array}{l}\text { Pearson } \\
\text { correlation }\end{array}$ & $\begin{array}{l}\text { Regression } \\
\text { value at } \mathbf{2 7} \text { wk } \\
\text { [95\% confidence } \\
\text { interval] }\end{array}$ & $\begin{array}{l}\text { Regression } \\
\text { value at } \mathbf{3 7} \mathbf{w k} \\
\text { [95\% confidence } \\
\text { interval] }\end{array}$ & $\begin{array}{l}\text { b-coefficient } \\
\text { [95\% confi- } \\
\text { dence interval] }\end{array}$ \\
\hline Frontal (Fp1-Fp2) & $0.62\left(^{*}\right)$ & $32[29 ; 36]$ & $18[14 ; 22]$ & $-1.5[-2.1 ;-0.8]$ \\
\hline Central (C3-C4) & 0.26 (NS) & $\mathrm{n} / \mathrm{a}$ & $\mathrm{n} / \mathrm{a}$ & $\mathrm{n} / \mathrm{a}$ \\
\hline Occipital (O1-O2) & 0.11 (NS) & $\mathrm{n} / \mathrm{a}$ & $\mathrm{n} / \mathrm{a}$ & $\mathrm{n} / \mathrm{a}$ \\
\hline Centro-frontal right (C4-Fp2) & $0.67\left(^{*}\right)$ & $32[28 ; 35]$ & $15[10 ; 19]$ & $-1.7[-2.4 ;-1.0]$ \\
\hline Centro-frontal left (C3-Fp1) & $0.62\left(^{*}\right)$ & $28[25 ; 32]$ & $13[9 ; 18]$ & $-1.5[-2.2 ;-0.8]$ \\
\hline Centro-temporal right (C4-T4) & $0.60\left(^{*}\right)$ & $40[33 ; 46]$ & $15[8 ; 22]$ & $-2.5[-3.6 ;-1.3]$ \\
\hline Centro-temporal left (C3-T3) & $0.61(*)$ & $38[32 ; 44]$ & $13[6 ; 21]$ & $-2.5[-3.6 ;-1.3]$ \\
\hline Centro-occipital right (C4-O2) & 0.31 (NS) & $\mathrm{n} / \mathrm{a}$ & $\mathrm{n} / \mathrm{a}$ & $\mathrm{n} / \mathrm{a}$ \\
\hline Centro-occipital left (C3-O1) & 0.34 (NS) & $\mathrm{n} / \mathrm{a}$ & $\mathrm{n} / \mathrm{a}$ & $\mathrm{n} / \mathrm{a}$ \\
\hline \multicolumn{5}{|c|}{$\begin{array}{l}\text { Table } 4 \text { shows the Pearson correlation coefficient and linear regression analysis between postmenstrual age (PMA) an } \\
\text { bandwidth values of the aEEG for nine different channels. The regression values (bandwidth, } \mu \mathrm{V} \text { ) with } 95 \% \text { confidenc } \\
\text { intervals are calculated for } 27 \text { and } 37 \text { weeks of gestation, respectively. The b-coefficient (slope) refers to the change o } \\
\text { bandwidth per week ( } \mu \mathrm{V} / \mathrm{wk} \text { ). } \\
\left({ }^{*}\right) \text { P-value }<0.005 ;\left(^{* *}\right) \text { P-value }<0.05 \text {; NS refers to not significant; } \text { n/a not applicable. }\end{array}$} \\
\hline
\end{tabular}

ranging from 33 to 42 and $19-22 \mu \mathrm{V}$, respectively. The b-coefficient, indicating UMA change per week, varied between -1.3 and $-2.2 \mu \mathrm{V} /$ week and was not different between the channels.

\section{Bandwidth (Table 4)}

Like the upper margin amplitude, we observed strong positive correlations (varying between +0.59 and +0.66; $\mathrm{p}<0.01$ ) between PMA and bandwidth for the frontal, centro-frontal and centro-occipital channels. The regression values calculated for 27 and 37 weeks of PMA were comparable between these channels, with values ranging from 29 to 39 and $13-17 \mu \mathrm{V}$, respectively. The b-coefficient, indicating bandwidth change per week, varied between -1.5 and $-2.4 \mu \mathrm{V} /$ week and was not different between the channels.

\section{Quantification of aEEG patterns}

The statistical distribution of amplit ude values within each aEEG recording was expressed in skewness of data. Figure 2 shows the correlation between PMA and skewness of LMA values of the central channel $\left(\mathrm{C}_{3}-\mathrm{C}_{4}\right)$. In general, skewness was between +1 and -1 , indicating a normal distribution. However, below 32 weeks of gestation a positive skewness was observed, while a negative skewness was found above 32 weeks of gestation. In general, all channels showed significant relationships between PMA and skewness (median regression coefficient, -0.70 ; range -0.51 to-0.87). Hence, very immature aEEG patterns can be quantified by a relative low baseline (LMA values of $b_{5} \mu \mathrm{V}$ ) with incidental bursts of activity (reflected by positive skewness), while aEEG 
patterns towards term are characterized by a higher baseline (LMA values $\mathrm{N}_{5} \mu \mathrm{V}$ ) and incidental decreases of activity (reflected by negative skewness) (figure 3).

Figure 2:

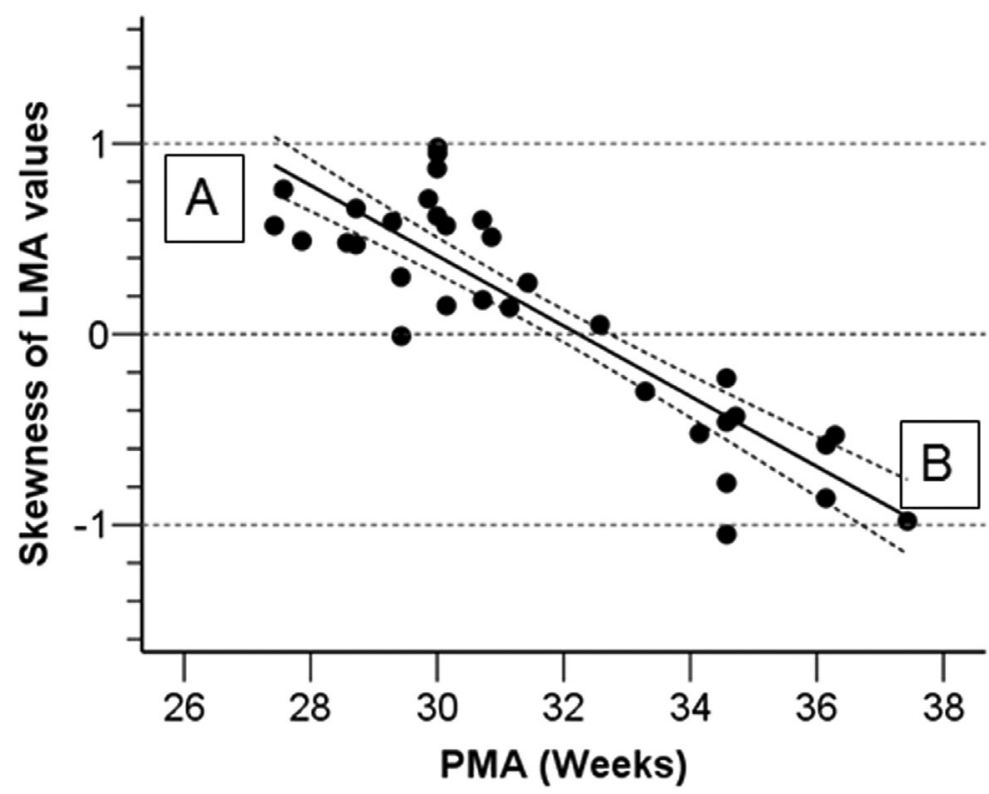

Figure 2 shows the correlation (regression coefficient, - 0.87) between postmenstrual age (PMA, wk) and statistical distribution of lower amplitude values (expressed in skewness values, no dimension) of the central channel $\left(C_{3}-C_{4}\right)$ for each of the 40 aEEG recordings. In general, below 32 weeks of gestation a positive skewness was observed ( $A$, see also Figure 3 ), whilst a negative skewness was found above 32 weeks of gestation ( $B$, see also Figure 3 ).

\section{Discussion}

This is the first study, where multi-channel aEEG aspects were studied by computer analysis. In all channels we observed an increase of continuous electrocortical background activity, reflected by LMA, with PMA. From 27 to 37 weeks of PMA, LMA increased approximately $3-4 \mu \mathrm{V}$. The lowest LMA values were found in the occipital and frontal channels, the highest values in the centrooccipital channels. The central $\left(\mathrm{C}_{3}-\mathrm{C}_{4}\right)$ channel, close to the biparietal $\left(\mathrm{P}_{3}-\mathrm{P}_{4}\right)$ lead placement of single channel aEEG, showed changes in LMA values with PMA, but not in UMA or bandwidth. We found symmetric changes in the aEEG characteristics between the left and right hemispheres. In addition, skewness of LMA values strongly correlates with PMA, a positive skewness indicating an immature brain and a negative skewness a maturing brain. 
Figure 3:
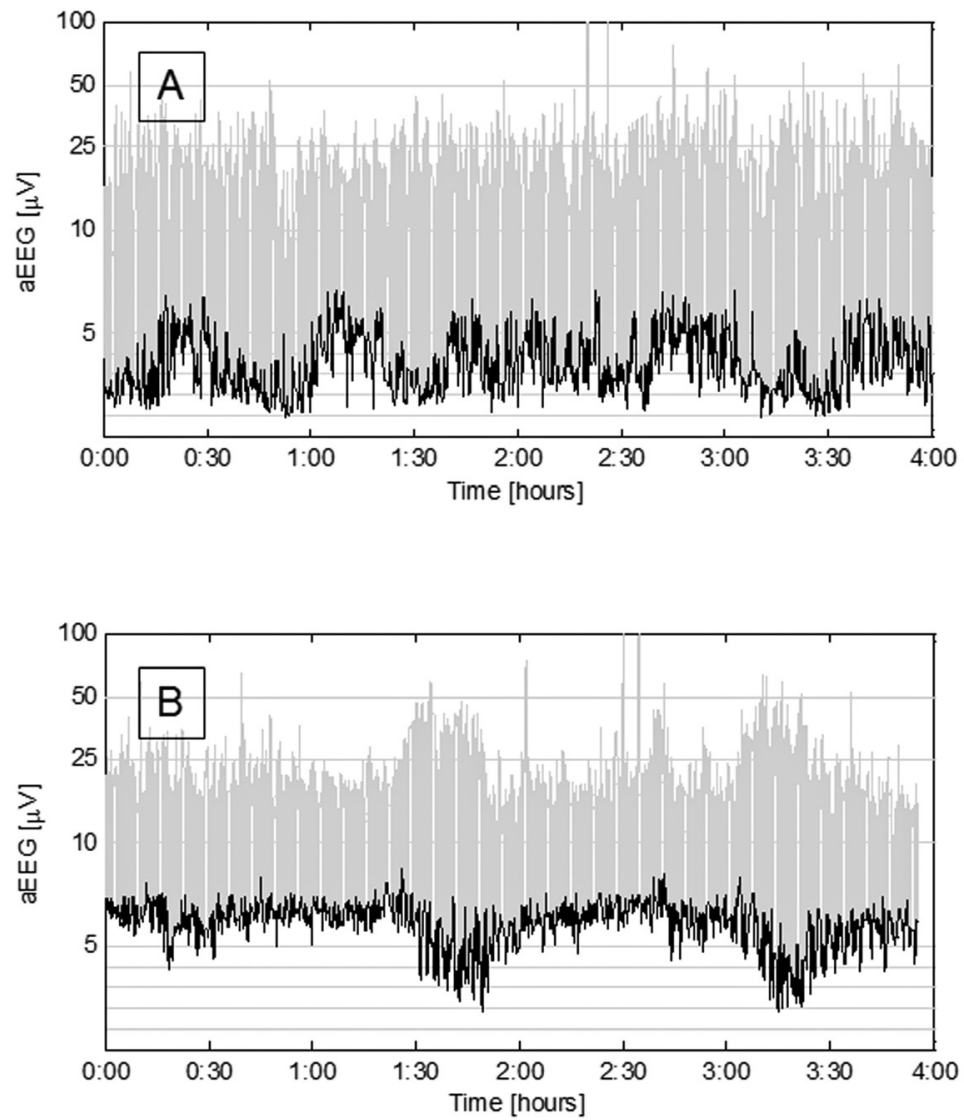

Figure 3 illustrates the differences in aEEG pattern of preterm infants by lower amplitude values. Note, that the $y$-axis is semi-logarithmic, linear between 0-10 $\mu \mathrm{V}$ and logarithmic between 10-100 $\mu \mathrm{V}$. Very immature aEEG patterns (panel A) can be quantified by a relative low baseline (LMA values of $1-2 \mu \mathrm{V}$ ) with incidentally burst of activity (reflected by positive skewness), while aEEG patterns towards term (panel B) is characterized by a higher baseline (LMA values of 6-7 $\mu \mathrm{V}$ ) and incidentally decreases of activity (reflected by negative skewness). Pattern B is consistent with sleep-wake cycles.

\section{aEEG maturation}

Studies on electrophysiological human brain maturation show that the preterm brain matures from a discontinuous trace (with short bursts of activity alternated with long periods of interburst intervals in which there is very low electrical activity) to a continuous trace (with many bursts of activity and short periods of interburst intervals) [26]. On aEEG this transition is reflected by a rise in LMA and the development of sleep-wake cycling [10]. A discontinuous trace shows little EEG activity and therefore a lower LMA in aEEG signal than a continuous trace [16, 27].We demonstrated that this transition in background activity of the aEEG with age is reflected very well by changes in skewness of LMA values. There are several hypotheses about why continuity and LMA increase 
with increasing PMA. Biagioni et al. suggested that increasing electrical activity is related to the cortical folding process [8]. Other authors suggested that increasing EEG continuity advances with the maturation of excitatory synapses [28].

Although several studies found an overall increase of EEG continuity with advancing PMA, there remains a great variability in reference values for voltages and duration of normal background patterns. This is especially true for EEG studies, in which mainly qualitative or semi-quantitative techniques have been used. Olischar et al. used a variable distinction of a discontinuous low voltage pattern $(<3 \mu \mathrm{V})$ in very preterm infants (24-25 weeks) and a discontinuous high voltage pattern $(3-5 \mu \mathrm{V})$ in preterm infants of $26-27$ weeks gestation [15]. Also we found LMA values $\leq 5 \mu \mathrm{V}$ in preterm infants with a PMA $<32$ weeks.

Notably, we did not find consistent correlations between PMA and UMA or bandwidth for the central channel. Thus, single-channel aEEG over the biparietal areas (close to the central channel) may not reflect all maturational changes. This is in agreement with a study from Sisman et al. [16]. UMA and bandwidth are important characteristics often used in the assessment of sleep-wake cycling in which short periods of a tracing are assessed $[29,30]$. The longitudinal centro-temporal or centro-frontal channels are better candidates to assess changes in bandwidth.

\section{Regional differences}

We detected differences of 1 to $2 \mu \mathrm{V}$ between the channels, with the lowest LMA values in the frontal and occipital channels. As the clinical aEEG is displayed linearly in the $0-10 \mu \mathrm{V}$ range, differences of $1 \mu \mathrm{V}$ can be noted visually. Higher LMA values in the central region may indicate more continuous electrocortical activity compared to the occipital and frontal regions. These observations may partly be explained by differences in inter-electrode distances [31]. In the reduced 10-20 montage system for neonates the inter-electrode distance between the occipital or frontal electrodes, and central electrodes $\left(\mathrm{C}_{3}-\mathrm{C}_{4}\right)$ is 20 and $40 \%$ of the diameter of the head, respectively. However, as no differences in LMA values were found between the centro-temporal (equal to $20 \%$ of diameter) and centro-frontal (40\%) channels, we assume that inter-electrode distances may only partly explain our observations.

Differences of LMA values between channels may indicate differences in brain maturation. MRI studies show that the earliest gyration takes place at the central area and that the maturation of the occipital region precedes that of the other brain regions [32, 33]. We did not perform MRI in our subjects and therefore cannot draw straight conclusions about the relationship of sulcus maturation and aEEG.

\section{Symmetry}

Besides discontinuity and upper and lower margin amplitudes, inter-hemispheric asynchrony or asymmetry have been suggested to be useful characteristics in assessing brain dysmaturity and brain dysfunction [34-36]. Where inter-hemispheric synchrony suggests resemblance of brain activity in time, inter-hemispheric symmetry gives information about resemblance in frequency and amplitude between left and right [35]. Analysis of EEG recordings in full-term infants has shown that cerebral activity is generally symmetric, while marked amplitude asymmetries during substantial part of the recording are indicative of severe pathology [37]. 
We examined symmetry as measured by aEEG by taking into consideration the entire background. The difference of mean LMAbetween hemispheres with increasing PMA was assessed. Our data showed a symmetrical increase of LMA and decrease in UMA or band-width with PMA between left and right hemispheres. This is in accordance with previous EEG studies who found symmetric EEG signals in neurologically healthy infants $[35,38]$.

This is the first study to assess symmetry in brain maturation using automated multi-channel aEEG. We confirm the finding that overall brain maturation in neurologically stable preterm infants is symmetrical for both hemispheres. Research needs to be extended to preterm infants with brain injury, because this may have clinical implications. Indeed, in term newborns with unilateral brain damage two-channel aEEG recordings are recommended [22]. If preterm infants with brain injury have marked asymmetry on aEEG, we advocate using the reduced 10-20 EEG montage system to improve identification.

\section{Methodological considerations}

First, the number of preterm infants is relatively small. Of 230 eligible preterm infants, 51 enrolled in the study and after exclusion of 11 (complicated neonatal course or delayed neurodevelopment at 2 years of age), 40 were finally analyzed. The aim of this study was to assess aEEG characteristics in healthy preterm infants without any additional pathologic conditions that might interfere with normal cerebral development. This is an important issue, as severity of illness and hypotension are associated with suppression of aEEG background activity [39]. In addition, carbon dioxide and plasma glucose level influence EEG activity in extremely preterm infants, and values considered to be within normal physiologic ranges are associated with the best EEG background [40]. Hence, we consider that the selection procedure, including only preterm infants who were clinically stable with normal blood pressure values and glucose, $\mathrm{pH}$ and base excess levels within the physiologic range as a strength. Thus, aEEG characteristics are obtained in the "healthy" preterm infant.

Second, the subjects showed normal neurodevelopment at 2 years of age. The high risk preterm infants with a gestational age $<32$ weeks showed normal follow-up according to Bayley Scales of Infant Development for mental and motor function at 24 months of corrected age. Although the low-risk infants were not assessed by the Bayley Scales, we consider a normal psychomotor development at the age of 3, 6, 12 and 24 months according to the Van Wiechen screening test adequate enough to exclude abnormal neurodevelopmental outcome.

Third, this study did not address sleep state of preterm infants. There was no differentiation between sleep states during quantitative analysis. To maximize robustness of the method we refrained from performing analysis on subsegments. Finally, for ethical reasons no (extra) simultaneous registration of $\mathrm{P}_{3}-\mathrm{P}_{4}$ was performed during the reduced $10-20$ montage EEG. However, as the closest corresponding electrode pair of the reduced 10-20 montage system is the adjacent central area, represented by the electrode pair $\mathrm{C}_{3}-\mathrm{C}_{4}$, we assume little or no differences between these channels [41]. 


\section{Conclusion}

By means of an automated analysis algorithm we quantitatively assessed several characteristics of the aEEG in clinical stable preterm infants with a normal follow-up at two years of corrected age. In all brain regions we observed a robust increase of LMA values with PMA. We observed symmetric increase of aEEG characteristics, indicating symmetric brain maturation of the left and right hemispheres.

With advancing PMA, the skewness of LMA values within the aEEG changes from a positive to a negative distribution, thereby reflecting maturation of background activity. Our findings demonstrate the clinical potential of computer-assisted analyses of aEEG recordings in detecting maturational (signal analytic) features which are no readily identified visually. This may provide an objective and reproducible method for assessing brain maturation and long-term prognosis. 


\section{$\underline{\text { References }}$}

1. Cooke RW. Preterm mortality and morbidity over 25 years. Arch Dis Child Fetal Neonatal Ed 2006;91(4):F293-4.

2. Larroque B, Ancel PY, Marret S, Marchand L, Andre M, Arnaud C, et al. Neurodevelopmental disabilities and special care of 5-year-old children born before 33 weeks of gestation (the EPIPAGE study): a longitudinal cohort study. Lancet 2008;371(9615):813-20.

3. Perlman JM. Neurobehavioral deficits in premature graduates of intensive care--potential medical and neonatal environmental risk factors. Pediatrics 2001;108(6):1339-48.

4. Klebermass $K$, Olischar M, Waldhoer T, Fuiko $R$, Pollak A, Weninger M. Amplitude-integrated EEG pattern predicts further outcome in preterm infants. Pediatr Res 2011;70(1):102-8.

5. Volpe JJ. Electroencephalography may provide insight into timing of premature brain injury. Pediatrics 2009;124(3):e542-4.

6. Tekgul H, Bourgeois BF, Gauvreau K, Bergin AM. Electroencephalography in neonatal seizures: comparison of a reduced and a full 10/20 montage. Pediatr Neurol 2005;32(3):155-61.

7. Andre M, Lamblin MD, d'Allest AM, CurziDascalova L, Moussalli-Salefranque F, TSNT, et al. Electroencephalography in premature and full-term infants. Developmental features and glossary. Neurophysiol Clin 2010;40(2):59-124.

8. Biagioni E, Frisone MF, Laroche S, Kapetanakis BA, Ricci D, Adeyi-Obe M, et al. Maturation of cerebral electrical activity and development of cortical folding in young very preterm infants. Clin Neurophysiol 2007;118(1):53-9.

9. ScherMS. Ontogeny of EEG sleep from neonatal through infancy periods. Handb Clin Neurol 2008;98:111-29.

10. Hellstrom-Westas L, Rosen I. Continuous brain-function monitoring: state of the art in clinical practice. Semin Fetal Neonatal Med
2006;11(6):503-11.

11. Rosen I. The physiological basis for continuous electroencephalogram monitoring in the neonate. Clin Perinatol 2006;33(3):593-611, v.

12. al Naqeeb N, Edwards AD, Cowan FM, AzzopardiD. Assessment of neonatal encephalopathy by amplitude-integrated electroencephalography. Pediatrics 1999;103(6 Pt 1):1263-71.

13. Toet MC, van der Meij W, de Vries LS, Uiterwaal CS, van Huffelen KC. Comparison between simultaneously recorded amplitude integrated electroencephalogram (cerebral function monitor) and standard electroencephalogram in neonates. Pediatrics 2002;109(5):772-9.

14. Klebermass K, Kuhle S, Olischar M, Rucklinger E, Pollak A, Weninger M. Intra- and extrauterine maturation of amplitude-integrated electroencephalographic activity in preterm infants younger than 30 weeks of gestation. Biol Neonate 2006;89(2):120-5.

15. Olischar M, Klebermass K, Kuhle S, Hulek M, Kohlhauser C, Rucklinger E, et al. Reference values for amplitude-integrated electroencephalographic activity in preterm infants younger than 30 weeks' gestational age. Pediatrics 2004;113(1 Pt 1):e61-6.

16. Sisman J, Campbell DE, Brion LP. Amplitudeintegrated EEG in preterm infants: maturation of background pattern and amplitude voltage with postmenstrual age and gestational age. J Perinatol 2005;25(6):391-6.

17. West $C R$, Harding JE, Williams CE, Gunning MI, Battin MR. Quantitative electroencephalographic patterns in normal preterm infants over the first week after birth. Early Hum Dev 2006;82(1):43-51.

18. Verma UL, Archbald F, Tejani NA, Handwerker SM. Cerebral function monitor in the neonate. I: Normal patterns. Dev Med Child Neurol 1984;26(2):154-61. 
19. Viniker DA, Maynard DE, Scott DF. Cerebral function monitor studies in neonates. Clin Electroencephalogr 1984;15(4):185-92.

20. Burdjalov VF, Baumgart S, Spitzer AR. Cerebral function monitoring: a new scoring system for the evaluation of brain maturation in neonates. Pediatrics 2003;112(4):855-61.

21. Niemarkt HJ, Andriessen P, Peters CH, Pasman JW, Blanco CE, Zimmermann LJ, et al. Quantitative analysis of amplitude-integrated electroencephalogram patterns in stable preterm infants, with normal neurological development at one year. Neonatology 2010;97(2):175-82.

22. van Rooij $L G$, de Vries $L S$, van Huffelen $A C$, Toet MC. Additional value of two-channel amplitude integrated EEG recording in fullterm infants with unilateral brain injury. Arch Dis Child Fetal Neonatal Ed 2010;95(3):F160-8.

23. Bourez-Swart MD, van Rooij L, Rizzo C, de Vries LS, Toet MC, Gebbink TA, et al. Detection of subclinical electroencephalographic seizure patterns with multichannel amplitude-integrated EEG in full-term neonates. Clin Neurophysiol 2009;120(11):1916-22.

24. The CRIB (clinical risk index for babies) score: $a$ tool for assessing initial neonatal risk and comparing performance of neonatal intensive care units. The International Neonatal Network. Lancet 1993;342(8865):193-8.

25. Brouwers-de Jong EA, Burgmeijer RJF, Laurent de Angelo MS. Ontwikkelingsonderzoek op het consultatiebureau: handboek bij het vernieuwde Van Wiechenonderzoek. Assen: Van Gorcum; 1996.

26. Niemarkt HJ, Andriessen P, Pasman J, Vles JS, Zimmermann LJ, Bambang Oetomo S. Analyzing EEG maturation in preterm infants: the value of a quantitative approach. J PerinatNeonatal Med 2008;1(3):131-44.

27. Hellstrom-Westas L. Continuous electroencephalography monitoring of the preterm infant. Clin Perinatol 2006;33(3):633-47, vi.
28. Vecchierini MF, Andre M, d'Allest AM. Normal EEG of premature infants born between 24 and 30 weeks gestational age: terminology, definitions and maturation aspects. Neurophysiol Clin 2007;37(5):311-23.

29. Kuint J, Turgeman A, Torjman A, MaayanMetzger A. Characteristics of amplitude-integrated electroencephalogram in premature infants. J Child Neurol 2007;22(3):277-81.

30. Osredkar D, Toet MC, van Rooij $L G$, van Huffelen AC, Groenendaal F, de Vries LS. Sleepwake cycling on amplitude-integrated electroencephalography in term newborns with hypoxic-ischemic encephalopathy. Pediatrics 2005;115(2):327-32.

31. Quigg $M$, Leiner D. Engineering aspects of the quantified amplitude-integrated electroencephalogram in neonatal cerebral monitoring. J Clin Neurophysiol 2009;26(3):145-9.

32. Dubois J, Benders M, Borradori-Tolsa C, Cachia A, Lazeyras F, Ha-Vinh Leuchter R, et al. Primary cortical folding in the human newborn: an early marker of later functional development. Brain 2008;131(Pt 8):2028-41.

33. van der Knaap MS, van Wezel-Meijler G, Barth PG, Barkhof F, Ader HJ, Valk J. Normal gyration and sulcation in preterm and term neonates: appearance on MR images. Radiology 1996;200(2):389-96.

34. Holmes GL, Lombroso CT. Prognostic value of background patterns in the neonatal EEG. J Clin Neurophysiol 1993;10(3):323-52.

35. Scher MS. Normal electrographic-polysomnographic patterns in preterm and fullterm infants. Semin Pediatr Neurol 1996;3(1):2-12.

36. Tharp BR. Electrophysiological brain maturation in premature infants: an historical perspective. J Clin Neurophysiol 1990;7(3):302-14.

37. Tharp BR, Cukier F, Monod N. The prognostic value of the electroencephalogram in premature infants. Electroencephalogr Clin Neurophysiol 1981;51(3):219-36. 
38. Selton D, Andre M, Hascoet JM. Normal EEG in very premature infants: reference criteria. Clin Neurophysiol 2000;111(12):2116-24.

39. ter Horst $H J$, Jongbloed-Pereboom $M$, van Eykern LA, Bos AF. Amplitude-integrated electroencephalographic activity is suppressed in preterm infants with high scores on illness severity. Early Hum Dev 2011;87(5):385-90.

40. Wikstrom S, Lundin F, Ley D, Pupp IH, Fellman V, Rosen I, et al. Carbon dioxide and glucose affect electrocortical background in extremely preterm infants. Pediatrics 2011;127(4):e1028-34.

41. Wusthoff CJ, Shellhaas RA, Clancy RR. Limitations of single-channel EEG on the forehead for neonatal seizure detection. J Perinatol 2009;29(3):237-42. 



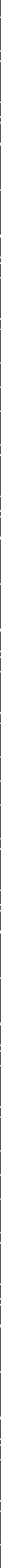




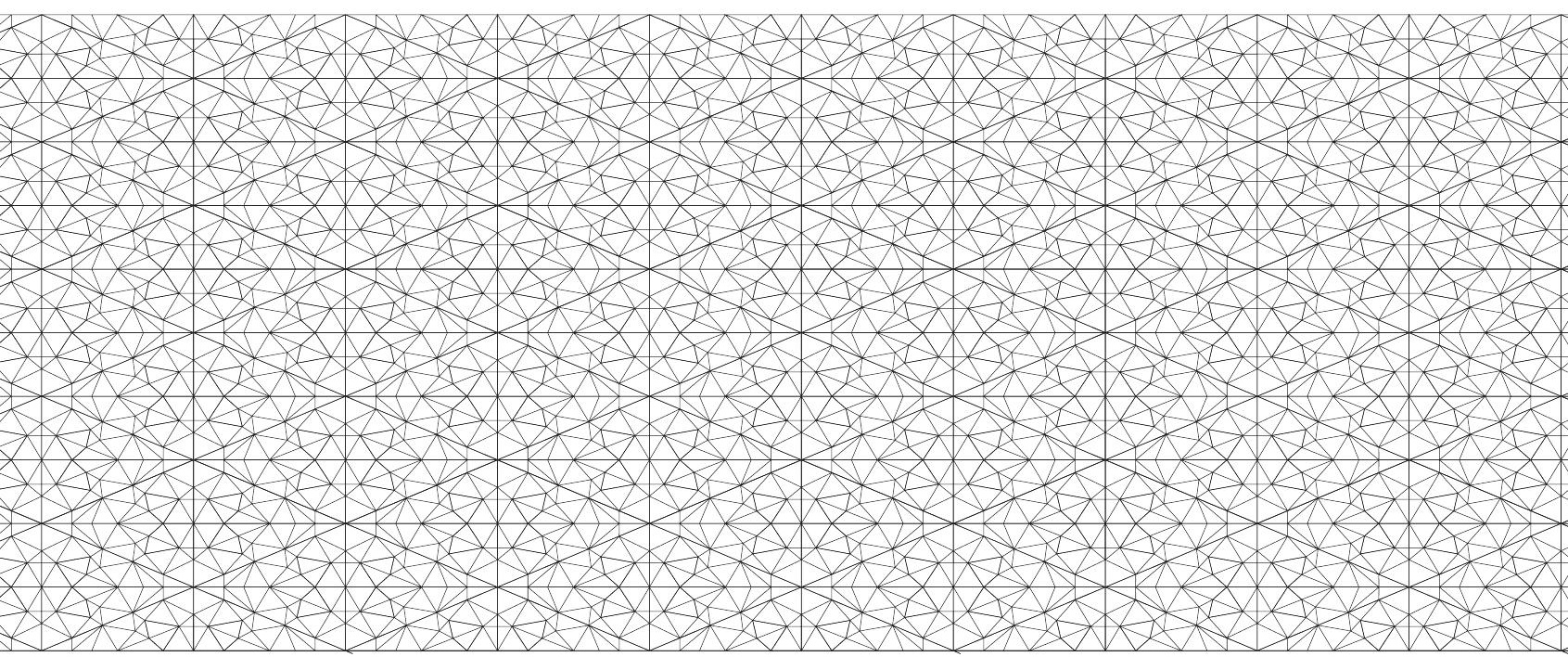




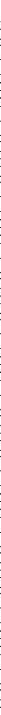

Discussion and future perspectives 


\section{$\underline{\text { Discussion and future perspectives }}$}

In this research project we performed serial multi-channel 4-hour electroencephalogram (EEG) recordings in a well selected preterm population. Serial EEG monitoring proved to be feasible in our NICU. Electrode placement took place without notable stress for the infant and acceptable impedances could almost always be reached. During the recording, electrodes remained at fixed position and impedance even decreased a little. EEG recording did not significantly interfere with daily routine. No complications of electrode placement or EEG recording were observed. One parent withdrew consent after enrollment in the study.

In our opinion, the strength of the study was related to inclusion criteria for defining a population of (healthy) preterm infants, the serial 4-hour EEG recordings and the follow-up data at the age of two years. The study project described in this thesis had two main objectives. First, we wanted to investigate if electrocortical maturation can be assessed by quantitative automated analysis. Second, we wanted to know which automated EEG markers are best to describe electrocortical maturation.

In chapter 2, we reviewed the maturation of the EEG in preterm infants and discussed which EEG aspects are suitable for quantitative automated analyses.

In chapter 3, we used the interburst-burst ratio (IBR) algorithm, provided by the Nicolette ${ }^{\circledR}$ EEG recorder to detect discontinuous and continuous background tracings. We found an increase in length of continuous tracings and a decrease in length of discontinuous tracings with progressing postmenstrual age. This was in concordance with earlier studies in which visual assessment of EEG tracings was performed. We observed - even in preterm infants of 27 weeks of gestation - a clear cycling pattern in EEG activity, suggestive for a rudimentary sleep-wake pattern. Besides, interburst intervals were automatically detected. Interburst interval length decreased with postmenstrual age. Mean IBR, which incorporates the length of discontinuous tracing as well as the length of interburst, may serve as an overall measure of discontinuity. IBR decreased with progressing postmenstrual age.

To detect EEG bursts and interburst intervals, we developed and validated an in-house algorithm in chapter 4. The optimal amplitude threshold to segregate burst from interburst interval was empirically determined at $30 \mu \mathrm{V}$. Bursts could be detected with a sensitivity of $90 \pm 6 \%$ and a positive predictive value of $88 \pm 8 \%$, while interburst intervals were detected with a sensitivity of $80 \pm 9 \%$ and a positive predictive value of $96 \pm 3 \%$. The optimal detection level for bursts and interburst intervals was $30 \mu \mathrm{V}$. Burst length did not significantly change with increasing postmenstrual age, while interburst length decreased (chapter 6 ). The length of the interburst intervals detected with our in-house algorithm showed a stronger correlation with postmenstrual age than the length of intervals detected with the Nicolette ${ }^{\circledast}$ monitor.

We studied the maturational change in EEG frequency aspects by automated spectral analysis in 4 -hour recordings in chapter $\mathbf{5}$. With progressing postmenstrual age, absolute delta powers and theta powers decreased. Relative delta 1 power decreased and relative alfa and beta power increased with postmenstrual age. Spectral edge frequency (defined as the frequency which delimits $95 \%$ of the power between 0.5 and $30 \mathrm{~Hz}$ ) increased with age. All the maturational changes in spectral frequency were most prominent in the centro-temporal channels. 
In chapter 6 we performed spectral analysis on the bursts after automated burst detection with our in house algorithm of chapter 4 . In this study we also paid more attention to the regional differences. Absolute burst powers decreased with postmenstrual age, while relative alfa and beta burst powers increased. Changes were most prominent on the temporal and frontal areas of the brain which corresponds with MRI studies showing ongoing gyration and postnatal white matter maturation in frontal and temporal lobes.

In chapter 7, automated analysis of the amplitude-integrated EEG (aEEG) background pattern was performed. With advancing postmenstrual age, an increase in lower margin amplitude (LMA) was observed, while bandwidth (BW) and upper margin amplitude (UMA) did not change significantly. Also, the time percentage of discontinuous background pattern decreased with progressing postmenstrual age. Our findings were in accordance with studies, in which aEEG was evaluated visually. Moreover, aEEG changes were equally influenced by gestational age and postnatal age. This negates the concept of differences between intra- and extra-uterine aEEG maturation in preterm infants, which was proposed by several investigators [1-4] but could not be reproduced by others [5].

In chapter 8, regional differences in aEEG maturation were studied in a group of patients with a wider postmenstrual age range than the earlier studies. Differences between the brain regions were small, with maximum difference in LMA of $2 \mu \mathrm{V}$. There was symmetry between the right and left hemisphere. Remarkably, BW and UMA changes were observed in some brain regions. Besides, the maturational increase in LMA and development of sleep-wake cycling, which lead to a change in the (asymmetric) distribution of LMA values or skewness, could be assessed by automated analysis. The skewness of LMA values showed a strong correlation with postmenstrual age. This is a good example of a maturational effect, which cannot be recognized by visual inspection only.

The second objective was related to evaluate which automated EEG parameters are appropriate to describe electrocortical maturation. Table 1 summarizes several EEG and amplitude-integrated EEG parameters which were found to correlate with postmenstrual age. Interburst interval length, aEEG lower margin amplitude (LMA), skewness of LMA, absolute and relative delta power, and relative alfa and beta power showed the highest correlation with postmenstrual age. Interburst interval length and LMA represent changes in EEG discontinuity. LMA and corresponding skewness represent the change in discontinuity and the evolvement of the sleep wake cycle. The spectral EEG changes describe the maturational increase in EEG frequencies. In several studies multiple EEG channels were investigated. Although maturational changes were found in almost all the channels, the central $\left(\mathrm{C}_{3}-\mathrm{C}_{4}\right)$ and de centro-temporal $\left(\mathrm{C}_{3}-\mathrm{T}_{3} ; \mathrm{C}_{4}-\mathrm{T}_{4}\right)$ channels were found to be superior in estimating neurophysiologic maturation.

\section{Considerations}

During our project we encountered several theoretical and practical challenges and limitations.

First, the definition of a healthy preterm neonate and normal neurophysiologic development of preterm infants may be a contradiction in terms as prematurity is a pathologic condition in 
Table 1: Different parameters of electrocortical maturation.

\begin{tabular}{|c|c|c|c|c|}
\hline Marker & Location & Analysis technique & Value & p value \\
\hline $\begin{array}{l}\text { Interburst-Burst-Ratio } \\
\text { (IBR) }\end{array}$ & $\mathrm{C} 3-\mathrm{C} 4$ & Logarithmic regression & $R^{2}=0.64$ & $\mathrm{p}<0.001$ \\
\hline $\begin{array}{l}\text { Interburst Interval (IBI) } \\
\text { (Nicolette) }\end{array}$ & $\mathrm{C} 3-\mathrm{C} 4$ & Linear regression & $R^{2}=0.43 / r=-0,66$ & $\mathrm{p}<0.001$ \\
\hline $\begin{array}{l}\text { Length discontinuous } \\
\text { activity }\end{array}$ & $\mathrm{C} 3-\mathrm{C} 4$ & Linear regression & $R^{2}=0.38 / r=-0.68$ & $p<0.001$ \\
\hline $\begin{array}{l}\text { Length continuous } \\
\text { activity }\end{array}$ & $\mathrm{C} 3-\mathrm{C} 4$ & Linear regression & $R^{2}=0.50 / r=0.70$ & $\mathrm{p}<0.001$ \\
\hline $\begin{array}{l}\text { Interburst interval (IBI) } \\
\text { length }\end{array}$ & $\mathrm{C} 3-\mathrm{C} 4$ & Linear regression & $r=0.76$ & $P<0.01$ \\
\hline Burst length & $\mathrm{C} 3-\mathrm{C} 4$ & Linear regression & $r=0.02$ & NS \\
\hline \multirow[t]{3}{*}{ Absolute delta 1 power } & $\mathrm{C} 3-\mathrm{C} 4$ & Multilevel & $\begin{array}{l}a=280 \pm 80 \\
b=-6 \pm 2\end{array}$ & $\mathrm{p}<0.01$ \\
\hline & C3-T3 & Multilevel & $\begin{array}{l}a=620 \pm 90 \\
b=-16 \pm 3\end{array}$ & $p<0.01$ \\
\hline & C4-T4 & Multilevel & $\begin{array}{l}a=720 \pm 80 \\
b=-18 \pm \pm 3\end{array}$ & $\mathrm{p}<0.01$ \\
\hline \multirow[t]{3}{*}{ Absolute delta 2 power } & $\mathrm{C} 3-\mathrm{C} 4$ & Multilevel & $\begin{array}{l}a=130 \pm 40 \\
b=-3 \pm 1\end{array}$ & $\mathrm{p}<0.01$ \\
\hline & C3-T3 & Multilevel & $\begin{array}{l}a=210 \pm 40 \\
b=-5 \pm 1\end{array}$ & $\mathrm{p}<0.01$ \\
\hline & C4-T4 & Multilevel & $\begin{array}{l}a=270 \pm 40 \\
b=-6 \pm 1\end{array}$ & $p<0.01$ \\
\hline \multirow[t]{3}{*}{ Relative delta 1} & $\mathrm{C} 3-\mathrm{C} 4$ & Multilevel & $\begin{array}{l}a=88 \pm 7 \\
b=-1.0 \pm 0.2\end{array}$ & $p<0.01$ \\
\hline & C3-T3 & Multilevel & $\begin{array}{l}a=103 \pm 8 \\
b=-1.50 .2\end{array}$ & $\mathrm{p}<0.01$ \\
\hline & C4-T4 & Multilevel & $\begin{array}{l}a=98 \pm 7 \\
b=1.30 .2\end{array}$ & $\mathrm{p}<0.01$ \\
\hline \multirow[t]{2}{*}{ Relative alfa } & $\mathrm{C} 3-\mathrm{C} 4$ & Multilevel & $\begin{array}{l}a=-5 \pm 1 \\
b=0.23 \pm 0.03\end{array}$ & $\mathrm{p}<0.01$ \\
\hline & C3-T3 & Multilevel & $\begin{array}{l}a=-6 \pm 1 \\
b=0.25 \pm 0.04\end{array}$ & $\mathrm{p}<0.01$ \\
\hline
\end{tabular}




\begin{tabular}{|c|c|c|c|c|}
\hline Marker & Location & Analysis technique & Value & p value \\
\hline & C4-T4 & Multilevel & $a=-6 \pm 1$ & $\mathrm{p}<0.01$ \\
\hline & & & $b=0.25 \pm 0.03$ & \\
\hline \multirow[t]{6}{*}{ Relative beta } & C3-C4 & Multilevel & $a=-4 \pm 1$ & $p<0.01$ \\
\hline & & & $b=0.20 \pm 0.03$ & \\
\hline & C3-T3 & Multilevel & $a=-6 \pm 1$ & $p<0.01$ \\
\hline & & & $b=0.26 \pm 0.04$ & \\
\hline & C4-T4 & Multilevel & $a=-7 \pm 2$ & $p<0.01$ \\
\hline & & & $b=0.29 \pm 0.05$ & \\
\hline Lower Margin aEEG & $\mathrm{C} 3-\mathrm{C} 4$ & Pearson & $r=+0.66$ & $p<0.01$ \\
\hline Amplitude (LMA) & & & $r=+0.87$ & \\
\hline Upper Margin aEEG & Fp1-Fp2 & Pearson & $r=-0.54$ & $p<0.01$ \\
\hline \multicolumn{5}{|l|}{ Amplitude (UMA) } \\
\hline Bandwidth of aEEG (BW) & Fp1-Fp2 & Pearson & $r=-0.62$ & $p<0.01$ \\
\hline $\begin{array}{l}\text { Percentage Discontinu- } \\
\text { ous aEEG tracing (DC-\%) }\end{array}$ & $\mathrm{C} 3-\mathrm{C} 4$ & Pearson & $r=-0.71$ & $p<0.01$ \\
\hline Skewness of LMA & C3-C4 & Pearson & $r=-0.87$ & $p<0.01$ \\
\hline
\end{tabular}

itself. To overcome this, we applied stringent criteria in the selection of infants. Of the 95 eligible infants, 18 were included in the study. The clinical course of these infants was uneventful during the neonatal period, and the infants showed a normal follow up at 2 years of corrected age. The negative predictive value of the Bayley Scales Mental Development Index for cognitive functioning at school age is 0.99 [6]. Because of our stringent selection criteria and follow-up, we consider the study population as healthy. However, strict selection criteria have lead to a relatively small study population and therefore to rather large confidence intervals of normal values.

Second, the electroencephalogram of the preterm infant is not only influenced by the state of brain development, but also by many clinical conditions, e.g. medication [7, 8], blood pressure [9, 10], blood glucose, carbon dioxide [11, 12] or sleep state [13]. Beside these factors, major changes in the EEG are observed during the first days of life, which may reflect merely the adaptation to extra-uterine life[14]. Therefore, it may be difficult to decide whether an EEG parameter is attributed to brain development or is flawed by the current clinical condition. To detect chronic stage EEG abnormalities, i.e. dysmature EEG patterns, which are related with adverse neurocognitive outcome, serial recordings of a single patient are indicated [15]. When using the EEG for assessment of brain development, we advise to assess serial EEG recordings, which encompasses at least 3 sleep-wake cycles, and not only rely on recordings performed in the first days of life.

Third, gender may influence brain development and therefore electrocortical maturation. Indeed, there are sex differences in the EEG of term infants. Infra-slow activity is larger in boys, 
while higher frequencies are larger in girls [16]. This suggests advanced neuro-maturation in girls. This is also demonstrated in visual evoked potential studies, where girls showed shorter (more mature) latencies [17]. Beside functional differences, MRI studies have shown gender differences. Term boys have higher gray matter volumes [18]. This was also observed in preterm boys [19]. In contrast, in a study by Kapellou et al. preterm boys showed slower cortical development, which was associated with neurodevelopmental impairment at 2 years of age [20]. To date, sex differences have not been demonstrated in the EEG of preterm infants. Most of our studies had a male to female distribution of 60 to $40 \%$. Due to low number of infants included in our study we did not investigate sex differences.

Fourth, in our study population we did not observe any clinical or electrical seizure activity. In other recent EEG studies in preterm infants, electrocortical seizure activity was observed in varying percentages from 3 to $22 \%$ [21-23]. Seizure activity was linked to large intraventricular hemorrhages and adverse outcome. As we strictly selected our study population, the absences of seizures is comprehensible.

Fifth, artifacts may have influenced results in automated EEG analysis. Especially in infants EEG artifacts are a major concern (table 1). The EEG recorder filtered the raw EEG between $0.3 \mathrm{~Hz}$ and $30 \mathrm{~Hz}$, and contained a $50 \mathrm{~Hz}$ notch filter. The different analysis algorithms also filtered EEG on frequency aspects. However, artifacts still influenced results. For that reason, data with extreme amplitude values were automatically trimmed before analysis in some studies (chapter 5 and 6). This was never more than $5 \%$ of the total data. In a recent study by Schumacher et al. automated spectral analysis was performed in preterm infants at the first days of live [24]. Automated trimming of the data by $5 \%$ led to similar results as did visually editing of the EEG before analysis. However, artifact removal has to be performed with caution as preterm EEG activity contains higher and more variable EEG amplitudes than in adult patients [25]. Besides, this technique still has flaws, as artifacts with normal frequency and normal (wide) amplitude range are still incorporated in the definitive analysis. In order to detect and to remove artifacts, simultaneous recording of ECG, respiratory rate, muscular activity and video recording is advised [25]. The data of these signals (especially ECG and respiratory rate which are already monitored in the NICU) may be incorporated in an automated detection algorithm.

Sixth, amplitude-integrated EEG (aEEG) generally uses the parietal channel ( $\left.P_{3}-P_{4}\right)$. As we performed EEG with electrodes placed according to the 10-20 reduced montage system, $\mathrm{P}_{3}$ and $\mathrm{P}_{4}$ were left out. As aEEG parameters are dependent on the location they are registered, results cannot be extrapolated to standard $\mathrm{P}_{3}-\mathrm{P}_{4}$ aEEG recordings. Indeed, in a study by Quigg et al. mean aEEG values turned out to be $\pm 1,5 \mu \mathrm{V}$ higher at $\mathrm{C}_{3}-\mathrm{C}_{4}$ than $\mathrm{P}_{3}-\mathrm{P}_{4}$ [26]. However, because the central channel $\left(\mathrm{C}_{3}-\mathrm{C}_{4}\right)$ seems to be superior to detect convulsions in neonates, this may be the primary site for aEEG recording in the future $[27,28]$. The Brainz ${ }^{\circledast}$ monitor, which is a frequently used multichannel aEEG recorder, already makes use of electrodes placed at the $C_{3}$ and $C_{4}$ position. Other recent aEEG studies also made use of the $C_{3}$ and $C_{4}$ electrode positions $[29,30]$.

Moreover, quantitative analysis of the aEEG background pattern may be misleading as aEEG voltage may be influenced by scalp edema and inter-electrode distance. Furthermore, the lower margin amplitude (LMA) of the aEEG may be influenced by extra-cranial activity like ECG or high frequency ventilation, which leads to a "baseline-drift" of the LMA [31]. However, although the inter- 
Table 2: Mechanisms of disruption of brain maturation of preterm infants.

Encephalopathy of prema- Extra-uterine environment turity

\begin{tabular}{|c|c|c|c|}
\hline Mechanism & $\begin{array}{l}\text { Insults, related to the pre- } \\
\text { term birth, damage the brain, } \\
\text { which can be undetectable } \\
\text { by cerebral ultrasound. This } \\
\text { brain damage impairs further } \\
\text { brain development. }\end{array}$ & $\begin{array}{l}\text { Premature birth and the } \\
\text { (stressful) extra uterine envi- } \\
\text { ronment disrupts normal brain } \\
\text { development }\end{array}$ & $\begin{array}{l}\text { Preterm brain develop- } \\
\text { ment is secondary } \\
\text { influenced by the } \\
\text { extra-uterine environ- } \\
\text { ment }\end{array}$ \\
\hline MRI evidence & $\begin{array}{l}\text { Diffuse Excessive High Signal } \\
\text { Intensity (DEHSI) is related to } \\
\text { altered white matter organi- } \\
\text { zation }[20,49] \\
\text { White matter damage is } \\
\text { related to altered brain de- } \\
\text { velopment and neurological } \\
\text { outcome }[33,50]\end{array}$ & $\begin{array}{l}\text { In absence of white matter } \\
\text { abnormalities preterm infants } \\
\text { show at term age: } \\
\text { Less cortical gray matter and } \\
\text { white matter at term age [33] } \\
\text { Altered thalamic development } \\
\text { [32] } \\
\text { Altered cortical development } \\
\text { [34] } \\
\text { Brain development is dis- } \\
\text { rupted by Stress [37] } \\
\text { Neuroprotective strategies, } \\
\text { simulating intra-uterine envi- } \\
\text { ronment and reducing stress, } \\
\text { are linked to improved brain } \\
\text { development [38, 40] }\end{array}$ & $\begin{array}{l}\text { Brain development is } \\
\text { disrupted by } \\
\text { CLD [44] } \\
\text { Dexamethason [41] } \\
\text { Sepsis/NEC [51, 52] }\end{array}$ \\
\hline EEG evidence & $\begin{array}{l}\text { Absent aEEG cycling with the } \\
\text { first } 24 \mathrm{~h} \text { related to outcome } \\
\text { [30] } \\
\text { EEG discontinuity first } 48 \mathrm{~h} \\
\text { related to outcome [23] } \\
\text { Early EEG and aEEG disconti- } \\
\text { nuity related to outcome [53] }\end{array}$ & $\begin{array}{l}\text { Neuroprotective strategies } \\
\text { are related to accelerated EEG } \\
\text { maturation }[38,39,54]\end{array}$ & $\begin{array}{l}\text { EEG dysmaturity and } \\
\text { outcome are related to } \\
\text { CLD [46] } \\
\text { Nutrition [42] }\end{array}$ \\
\hline
\end{tabular}

Medical conditions

Medical conditions 
electrode distances of the centro-temporal and the centro-temporal channel significantly differ we found no difference in LMA between these two leads. Therefore, we suggest that inter-electrode distance only partly influences LMA. Besides, as visual interpretation of the aEEG background is also based on quantitative criteria, the conditions mentioned above may also disturb visual analysis. Nevertheless, to overcome the risk of disturbances on quantitative aEEG analysis, aEEG criteria, less influenced by shift of electrodes or baseline-drift, such as LMA skewness, can be analyzed. LMA skewness showed a very high correlation with PMA.

Finally, as mentioned before, the EEG of preterm infants is influenced by acute stage abnormalities (like hemorrhages, hypotension and carbon dioxide levels) and maturation. As the goal of our project was to develop a technique to monitor brain development, the focus was on EEG maturation and not on acute stage abnormalities. The brain development of preterm infants is at risk to be impaired. Even in the absence of intraventricular hemorrhages or periventricular leucomalacia, several studies demonstrate an impaired development in preterm infants. The degree of abnormalities in brain development is related to adverse neurocognitive outcome [32-34]. The mechanism of delayed brain development in the absence of detectable lesions remains to be clarified (table 2):

a. It is hypothesized that insults associated to preterm birth (hypoxia-ischemia, inflammation) damage the vulnerable immature brain and disrupt brain development, and that brain injury and impaired brain development are intertwined [35]. This damage may not be detected by cerebral ultrasonography. In MRI studies, diffuse excessive high signal intensity lesions of the white matter (DEHSI) have been linked to this 'encephalopathy of prematurity' concept [20]. However, as DEHSI is a subjective phenomenon with poor inter- and intra-observer agreement and is not clearly related to neurocognitive outcome, this concept is disputed by others [36]. The 'encephalopathy of prematurity' concept is supported by functional brain arguments: chronic stage EEG abnormalities are often preceded by acute stage abnormalities and EEG abnormalities in the first week of life are associated to neurodevelopmental outcome [15].

b. An alternative explanation for disruption normal brain maturation of preterm infants may be related to the extra-uterine environment. Maturing of a preterm infant in a stressful extrauterine environment may impair development. Even in preterm infants without white matter damage, gestational age remains the most predictive factor for outcome [32-34]. In EEG studies, extra-uterine life may accelerate (a)EEG maturation [1-4]. It has been demonstrated that stress impairs preterm brain development [37]. Neuro-protective strategies, aiming at simulating the intra-uterine environment and reduce stress, lead to a more physiologic EEG maturation $[38,39]$ and may protect brain development $[38,40]$.

c. Furthermore, it is plausible that several extra-uterine factors (e.g. dexamethason [41], nutrition and growth [42, 43], sepsis and chronic lung disease [44]) disrupt brain development [45]. In EEG studies, chronic dysmature EEG patterns are linked to this clinical conditions and neurocognitive outcome $[15,42,46,47]$.

It is conceivable that all three mechanisms of impaired brain development in preterm infants are complementary rather than contradictory to each other. Automated EEG analysis may help to diagnose brain maturation and to segregate normal from abnormal development. Our findings 
demonstrate the clinical potential of computer-assisted analyses of aEEG recordings in detecting maturational (signal analytic) features which are not readily identified visually.

Because of the multi-factorial influences on preterm brain maturation the best timing of EEG recording has to be determined. EEG recordings during the first days of life, a period crucial for brain development, reflects the adaptation to extra-uterine life rather than electrophysiological maturation [14]. As the secondary impact of extra-uterine life on brain development may take place at a later period, chronic stage EEG abnormalities (e.g. EEG dysmaturity) may be missed. Therefore, it seems inadequate to just monitor EEG during the first days of life. In a study of preterm infants $<30$ weeks of gestation, the pattern of EEG dysmaturity of sequential EEGs was associated with adverse neurological outcome [21]. Recently, Klebermass et al. demonstrated that aEEG patterns of preterm infants $<30$ weeks gestation correlated best with outcome if they were performed during the second week of life [48]. As we do not know exactly the etiology and mechanisms of (normal) brain maturation or the appropriate EEG characteristics, we advise to perform EEG recordings during the first days of life and perform weekly sequential recordings subsequently. Computer-assisted EEG analysis may provide an objective and reproducible method for assessing brain maturation and long-term prognosis.

\section{Future perspective}

Automated analysis makes the preterm EEG accessible for the neonatologist. It may lead to a more objective and uniform interpretation of the EEG and provide insight into preterm brain development. The amplitude-integrated EEG (aEEG) is a good example of computer-assisted analysis of the EEG and is increasingly being used in the preterm population. Indeed, an increasing amount of studies correlate preterm aEEG patterns with specific pathologic conditions and neurocognitive outcome [29, 30, 48, 53,55-58]. However, in these studies the background patterns are still analyzed by visual inspection and by different criteria. This leads to inter-observer variability, especially by non-experts [48]. The automated techniques which are performed in this research project may help in assessing aEEG background in an objective manner.

The following issues are important for future maturational EEG studies of preterm infants in the NICU:

1. As hemispheric symmetry and synchrony of the EEG is a sign of maturation and focal brain pathology (seizure activity) can be observed in more detail multi-channel (a)EEG analysis, I suggest to make use of a multi-channel (a)EEG monitor. Examples of devices are the Brainz ${ }^{\circledR}$ monitor (two channel, $\mathrm{C}_{3}-\mathrm{P}_{3}$ and $\mathrm{C}_{4}-\mathrm{P}_{4}$ ) and NicoletteOne (multi-channel, depend on montage of EEG). Our findings suggest that the central $\left(\mathrm{C}_{3}-\mathrm{C}_{4}\right)$ and both centro-temporal channels $\left(\mathrm{C}_{3}-\mathrm{T}_{3}\right.$ and $\left.\mathrm{C}_{4}-\mathrm{T}_{4}\right)$ are the best channels to monitor electrocortical maturation. Which channels are most practical to use may be subject for further research.

2. As the aEEG monitors record the raw EEG and process it, other automated raw EEG analyzing techniques, like interburst length measurement and spectral analyses may be implemented in the analysis. This will provide more information about neurophysiologic maturation. Indeed, the Brainz ${ }^{\circledast}$ monitor also displays an EEG continuity measure (defined as the time percentage 
during which EEG activity is above a certain threshold amplitude) [23]. The first efforts have been made to combine aEEG recording and spectral analysis [59]. The ideal (future) aEEG monitor displays the aEEG pattern, the original EEG signal, as well as several quantitative aEEG and EEG parameters. The following parameters show high correlation with postmenstrual age and represent important aspects of EEG maturation: lower margin amplitude with corresponding skewness and bandwidth (aEEG); interburst interval length (EEG) and limited spectral power information (absolute delta 1 power and/or relative alfa power). The parameters should be displayed with a time resolution of several hours to take into account several sleep-wake cycles.

3. In order to give more detailed estimation of neurophysiologic maturation, a multivariate logistic model which incorporates different EEG and aEEG aspects may be valuable. I propose to use the computer-assisted (a)EEG parameters which are found in our studies - containing amplitude and frequency criteria - for training a multivariate model [60]. Such a multivariate model for estimating electrocortical maturation may be tested and validated externally in a population of healthy preterm infants born in another perinatal centre.

4. Sleep-wake cycling is observed in very preterm infants [61-63]. This cycling may influence results of automated EEG analysis. The development of an automated sleep stage detection algorithm for preterm infants may be of value in a two-fold way: first, as sleep wake cycling is a sign of brain development and maturation [13, 30, 64], automated recognition of sleep wake cycling may assist in the assessment the brain function. Second, it may help in the automated selection of analyzed EEG epochs. Traditionally, analyzed EEG epochs are handpicked and results may be influenced by sleep state. When EEG patterns are always analyzed during a fixed sleep state or at least the sleep state is known, results will be more precise and show less variation. An automated sleep detecting algorithm for very preterm infants has to contain detection of so-called rapid eye movements and discontinuous EEG patterns, because these measures are related to early rudimentary sleep states $[61,63]$. Also, the ECG and the respiratory rate, which are already monitored at the NICU, can be implemented in a bedside (a)EEG monitor in order to detect sleep state and/or seizure activity.

If the present limitations of a computer-assisted and automated analysis of (amplitude-integrated) EEG become more 'mature' by future research and external validation, automated EEG analysis may become integrated in the intensive care of preterm infants. It may serve as a valuable tool for detection of pathologic conditions that interfere with normal neurodevelopment, for the prognosis of neurologic outcome and for the evaluation of neuroprotective strategies. 


\section{$\underline{\text { References }}$}

1. Klebermass K, Kuhle S, Olischar M, Rucklinger E, Pollak A, Weninger M. Intra- and extrauterine maturation of amplitude-integrated electroencephalographic activity in preterm infants younger than 30 weeks of gestation. Biol Neonate 2006;89(2):120-5.

2. LeeHJ, Kim HS, Kim SY, Sim GH, Kim ES, Choi CW, et al. Effects of postnatal age and aminophylline on the maturation of amplitude-integrated electroencephalography activity in preterm infants. Neonatology 2010;98(3):245-53.

3. Sisman J, Campbell DE, Brion LP. Amplitudeintegrated EEG in preterm infants: maturation of background pattern and amplitude voltage with postmenstrual age and gestational age. J Perinatol 2005;25(6):391-6.

4. Soubasi V, Mitsakis K, Nakas CT, Petridou S, Sarafidis K, Griva M, et al. The influence of extrauterine life on the aEEG maturation in normal preterm infants. Early Hum Dev 2009;85(12):761-5.

5. Kato T, Okumura A, Hayakawa F, Tsuji T, Natsume J, Watanabe K. Evaluation of brain maturation in pre-term infants using conventional and amplitude-integrated electroencephalograms. Clin Neurophysiol 2011;122(10):1967-72.

6. Hack M, Taylor HG, Drotar D, Schluchter M, Cartar L, Wilson-Costello D, et al. Poor predictive validity of the Bayley Scales of Infant Development for cognitive function of extremely low birth weight children at school age. Pediatrics 2005; 116(2):333-41.

7. Bernet V, Latal B, Natalucci G, Doell C, Ziegler A, Wohlrab G. Effect of sedation and analgesia on postoperative amplitude-integrated EEG in newborn cardiac patients. Pediatr Res 2010;67(6):650-5.

8. Supcun S, Kutz P, Pielemeier W, Roll C. Caffeine increases cerebral cortical activity in preterm infants. J Pediatr 2010;156(3):490-1.

9. Victor S, Marson AG, Appleton RE, Beirne M, Weindling AM. Relationship between blood pressure, cerebral electrical activity, cerebral fractional oxygen extraction, and peripheral blood flow in very low birth weight newborn infants. Pediatr Res 2006;59(2):314-9.

10. West $C R$, Groves $A M$, Williams $C E$, Harding JE, Skinner JR, Kuschel CA, et al. Early low cardiac output is associated with compromised elec troencephalographic activity in very preterm infants. Pediatr Res 2006;59(4 Pt 1):610-5.

11. Victor S, Appleton RE, Beirne M, Marson AG, Weindling AM. Effect of carbon dioxide on background cerebral electrical activity and fractional oxygen extraction in very low birth weight infants just after birth. Pediatr Res 2005;58(3):579-85.

12. Wikstrom S, Lundin F, Ley D, Pupp IH, Fellman V, Rosen I, et al. Carbon dioxide and glucose affect electrocortical background in extremely preterm infants. Pediatrics 2011;127(4):e1028-34.

13. ScherMS. Ontogeny of EEG sleep from neonatal through infancy periods. Handb Clin Neurol 2008;98:111-29.

14. West $C R$, Harding JE, Williams $C E$, Gunning MI, Battin MR. Quantitative electroencephalographic patterns in normal preterm infants over the first week after birth. Early Hum Dev 2006;82(1):43-51.

15. Okumura A, Hayakawa F, Kato T, Kuno K, Watanabe K. Developmental outcome and types of chronic-stage EEG abnormalities in preterm infants. Dev Med Child Neurol 2002;44(11):729-34.

16. Thordstein $M$, Lofgren $N$, Flisberg A, Lindecrantz K, Kjellmer I. Sex differences in electrocortical activity in human neonates. Neuroreport 2006;17(11):1165-8. 
17. Malcolm CA, McCulloch DL, Shepherd AJ. Pattern-reversal visual evoked potentials in infants: gender differences during early visual maturation. Dev Med Child Neurol 2002;44(5):345-51.

18. Gilmore $\mathrm{JH}$, Lin W, Prastawa MW, Looney $C B$, Vetsa YS, Knickmeyer RC, et al. Regional gray matter growth, sexual dimorphism, and cerebral asymmetry in the neonatal brain. $J$ Neurosci 2007;27(6):1255-60.

19. Dubois J, Benders M, Cachia A, Lazeyras F, Ha-Vinh Leuchter R, Sizonenko SV, et al. Mapping the early cortical folding process in the preterm newborn brain. Cereb Cortex 2008;18(6):1444-54.

20. Counsell SJ, Shen Y, Boardman JP, Larkman $D J$, Kapellou O, Ward P, et al. Axial and radial diffusivity in preterm infants who have diffuse white matter changes on magnetic resonance imaging at term-equivalent age. Pediatrics 2006;117(2):376-86.

21. Le Bihannic A, Beauvais K, Busnel A, de Barace $C$, Furby A. Prognostic value of EEG in very premature newborns. Arch Dis Child Fetal Neonatal Ed 2011.

22. Shah DK, Zempel J, Barton T, Lukas K, Inder TE. Electrographic seizures in preterm infants during the first week of life are associated with cerebral injury. Pediatr Res 2011;67(1):102-6.

23. West CR, Harding JE, Williams CE, Nolan M, Battin MR. Cot-side electroencephalography for outcome prediction in preterm infants: observational study. Arch Dis Child Fetal Neonatal Ed 2011;96(2):F108-13.

24. Schumacher EM, Westvik AS, Larsson PG, Lindemann R, Westvik J, Stiris TA. Feasibility of long-term continuous EEG monitoring during the first days of life in preterm infants: an automated quantification of the EEG activity. Pediatr Res 2011;69(5 Pt 1):413-7.

25. Walls-Esquivel E, Vecchierini MF, Heberle C, Wallois F. Electroencephalography (EEG) recording techniques and artefact detection in early premature babies. Neurophysiol Clin 2007;37(5):299-309.

26. Quigg M, Leiner D. Engineering aspects of the quantified amplitude-integrated electroencephalogram in neonatal cerebral monitoring. J Clin Neurophysiol 2009;26(3):145-9.

27. Bourez-Swart MD, van Rooij L, Rizzo C, de Vries LS, Toet MC, Gebbink TA, et al. Detection of subclinical electroencephalographic seizure patterns with multichannel amplitude-integrated EEG in full-term neonates. Clin Neurophysiol 2009;120(11):1916-22.

28. Shellhaas RA, Soaita Al, Clancy RR. Sensitivity of amplitude-integrated electroencephalography for neonatal seizure detection. Pediatrics 2007;120(4):770-7.

29. Kato T, Okumura A, Hayakawa F, Tsuji T, Natsume J, Hayakawa M. Amplitude-integrated electroencephalography in preterm infants with cystic periventricular leukomalacia. Early Hum Dev 2011;87(3):217-21.

30. Kidokoro H, Kubota T, Hayashi N, Hayakawa M, Takemoto K, Kato $Y$, et al. Absent cyclicity on aEEG within the first $24 \mathrm{~h}$ is associated with brain damage in preterm infants. Neuropediatrics 2010;41(6):241-5.

31. Toet MC, Lemmers PM. Brain monitoring in neonates. Early Hum Dev 2009;85(2):77-84.

32. Ball G, Boardman JP, Rueckert D, Aljabar P, Arichi T, Merchant N, et al. The Effect of Preterm Birth on Thalamic and Cortical Development. Cereb Cortex 2011.

33. Inder TE, Warfield SK, Wang H, Huppi PS, Volpe JJ. Abnormal cerebral structure is present at term in premature infants. Pediatrics 2005;115(2):286-94.

34. Kapellou O, Counsell SJ, Kennea N, Dyet L, Saeed N, Stark J, et al. Abnormal cortical development after premature birth shown by altered allometric scaling of brain growth. PLoS Med 2006;3(8):e265. 
35. Volpe JJ. The encephalopathy of prematurity-brain injury and impaired brain development inextricably intertwined. Semin Pediatr Neurol 2009;16(4):167-78.

36. Hart A, Whitby E, Wilkinson S, Alladi S, Paley M, Smith M. Neuro-developmental outcome at 18 months in premature infants with diffuse excessive high signal intensity on MR imaging of the brain. Pediatr Radiol 2011;41(10):1284-92.

37. Smith GC, Gutovich J, Smyser C, Pineda R, Newnham C, Tjoeng TH, et al. Neonatal intensive care unit stress is associated with brain development in preterm infants. Ann Neurol 2011.

38. Als H, Duffy FH, McAnulty GB, Rivkin MJ, Vajapeyam S, Mulkern RV, et al. Early experience alters brain function and structure. Pediatrics 2004;113(4):846-57.

39. Scher MS, Ludington-Hoe S, Kaffashi F, Johnson MW, Holditch-Davis D, Loparo KA. Neurophysiologic assessment of brain maturation after an 8-week trial of skin-to-skin contact on preterm infants. Clin Neurophysiol 2009;120(10):1812-8.

40. Milgrom J, Newnham C, Anderson PJ, Doyle LW, Gemmill AW, Lee K, et al. Early sensitivity training for parents of preterm infants: impact on the developing brain. Pediatr Res 2010;67(3):330-5.

41. Murphy BP, Inder TE, Huppi PS, Warfield $S$, Zientara GP, Kikinis $R$, et al. Impaired cerebral cortical gray matter growth after treatment with dexamethasone for neonatal chronic lung disease. Pediatrics 2001;107(2):217-21.

42. Okumura A, Hayakawa M, Oshiro M, Hayakawa F, Shimizu T, Watanabe K. Nutritional state, maturational delay on electroencephalogram, and developmental outcome in extremely low birth weight infants. Brain Dev 2010;32(8):613-8.

43. Ramenghi LA, Fumagalli M, Bassi L, Groppo M, De Carli A, Fanaro S, et al. Brain maturation of preterm newborn babies: new insights. J
Pediatr Gastroenterol Nutr 2007;45 Suppl 3:S143-6.

44. Thompson DK, Warfield SK, Carlin JB, Pavlovic $M$, Wang $H X$, Bear $M$, et al. Perinatal risk factors altering regional brain structure in the preterm infant. Brain 2007;130(Pt 3):667-77.

45. Perlman JM. Neurobehavioral deficits in premature graduates of intensive care--potential medical and neonatal environmental risk factors. Pediatrics 2001;108(6):1339-48.

46. Hahn JS, Tharp BR. Winner of the Brazier Award. The dysmature EEG pattern in infants with bronchopulmonary dysplasia and its prognostic implications. Electroencephalogr Clin Neurophysiol 1990;76(2):106-13.

47. ScherMS, Richardson GA, Salerno DG, Day NL, Guthrie RD. Sleep architecture and continuity measures of neonates with chronic lung disease. Sleep 1992;15(3):195-201.

48. Klebermass $K$, Olischar M, Waldhoer T, Fuiko R, Pollak A, Weninger M. Amplitude-integrated EEG pattern predicts further outcome in preterm infants. Pediatr Res 2011;70(1):102-8.

49. Skiold B, Horsch S, Hallberg B, Engstrom $M$, Nagy Z, Mosskin M, et al. White matter changes in extremely preterm infants, a populationbased diffusion tensor imaging study. Acta Paediatr 2010;99(6):842-9.

50. Woodward LJ, Anderson PJ, Austin NC, Howard $K$, Inder TE. Neonatal MRI to predict neurodevelopmental outcomes in preterm infants. $N$ Engl J Med 2006;355(7):685-94.

51. Hart AR, Whitby EH, Clark SJ, Paley MN, Smith MF. Diffusion-weighted imaging of cerebral white matter and the cerebellum following preterm birth. Dev Med Child Neurol 2010;52(7):652-9.

52. Shah DK, Doyle LW, Anderson PJ, Bear M, Daley AJ, Hunt RW, et al. Adverse neurodevelopment in preterm infants with postnatal sepsis or necrotizing enterocolitis is mediated by white matter abnormalities on magnetic resonance 
imaging at term. J Pediatr 2008;153(2):170-5, $175 \mathrm{e} 1$.

53. Wikstrom S, Ley D, Hansen-Pupp I, Rosen I, Hellstrom-Westas L. Early amplitude-integrated EEG correlates with cord TNF-alpha and brain injury in very preterm infants. Acta Paediatr 2008;97(7):915-9.

54. Guzzetta A, Baldini S, Bancale A, Baroncelli $L$, Ciucci F, Ghirri P, et al. Massage accelerates brain development and the maturation of visual function. J Neurosci 2009;29(18):6042-51.

55. Hellstrom-Westas L, Klette H, ThorngrenJerneck K, Rosen I. Early prediction of outcome with aEEG in preterm infants with large intraventricular hemorrhages. Neuropediatrics 2001;32(6):319-24.

56. Olischar M, Klebermass K, Kuhle S, Hulek M, Messerschmidt A, Weninger M. Progressive posthemorrhagic hydrocephalus leads to changes of amplitude-integrated EEG activity in preterm infants. Childs Nerv Syst 2004;20(1):41-5.

57. Chalak LF, Sikes NC, Mason MJ, Kaiser JR. Low-voltage aEEG as predictor of intracranial hemorrhage in preterm infants. Pediatr Neurol 2011;44(5):364-9.

58. El-Dib M, Massaro AN, Glass P, Bulas D, Badrawi $N$, Orabi A, et al. Early amplitude integrated electroencephalography and outcome of very low birth weight infants. Pediatr Int 2011;53(3):315-21.

59. Kobayashi K, Mimaki N, Endoh F, Inoue T, Yoshinaga H, Ohtsuka Y. Amplitude-integrated EEG colored according to spectral edge frequency. Epilepsy Res 2011.

60. Murray A, Glaria AP, Pearson DT. Monitoring EEG frequency and amplitude during cardiac surgery. Anaesthesia 1986;41(2):173-7.

61. Curzi-Dascalova L, Figueroa JM, Eiselt $M$, Christova E, Virassamy A, d'Allest AM, et al. Sleep state organization in premature infants of less than 35 weeks' gestational age. Pediatr Res 1993;34(5):624-8.
62. Olischar M, Klebermass K, Waldhoer T, Pollak A, Weninger M. Background patterns and sleep-wake cycles on amplitude-integrated electroencephalography in preterms younger than 30 weeks gestational age with peri-/ intraventricular haemorrhage. Acta Paediatr 2007;96(12):1743-50.

63. Scher MS, Johnson MW, Holditch-Davis D. Cyclicity of neonatal sleep behaviors at 25 to 30 weeks' postconceptional age. Pediatr Res 2005;57(6):879-82.

64. Kuhle S, Klebermass K, Olischar M, Hulek M, Prusa AR, Kohlhauser $C$, et al. Sleep-wake cycles in preterm infants below 30 weeks of gestational age. Preliminary results of a prospective amplitude-integrated EEG study. Wien Klin Wochenschr 2001;113(7-8):219-23. 



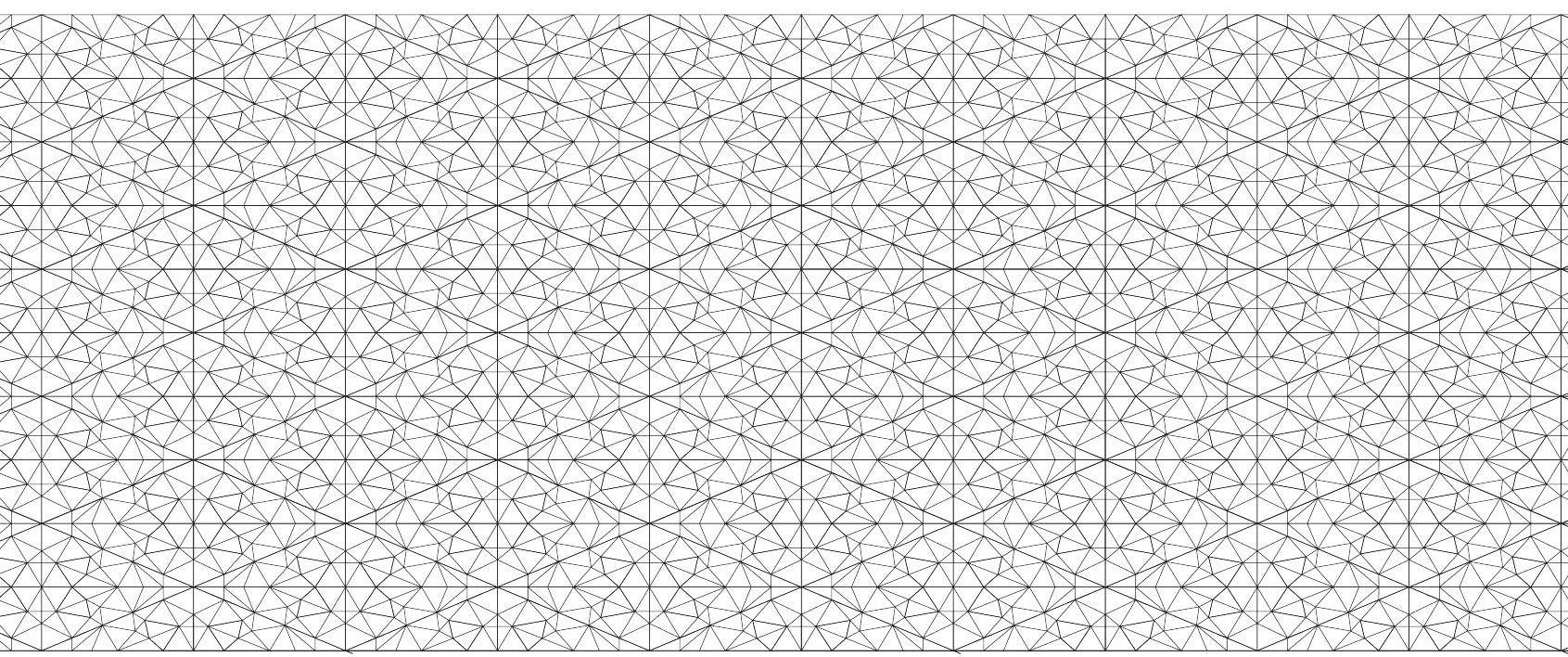




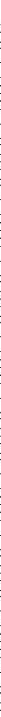

Nederlandse samenvatting 


\section{Nederlandse samenvatting}

Door de ontwikkelingen in de zorg voor te vroeg geboren kinderen zijn de overlevingskansen van deze kinderen de afgelopen decennia fors toegenomen. Desondanks blijft het percentage neurologische restverschijnselen hoog. De oorzaak van deze neurocognitieve problemen is nog altijd niet opgehelderd. Mogelijk wordt de ontwikkeling van het - zeer kwetsbare- brein van te vroeg geborenen verstoord tijdens het verblijf op de Neonatale Intensive Care Unit (NICU). Dit kan bijvoorbeeld plaatsvinden door medische omstandigheden (zuurstofgebrek, infectie), omgevingsfactoren (stress, licht, geluid) en ook bepaalde medische ingrepen. Het is daarom van belang om de hersenontwikkeling van vroeggeborenen op de NICU te monitoren.

Het klassieke elektroencephalogram (EEG) - ofwel hersenfilmpje - geeft ook bij te vroeg geboren kinderen de hersenfunctie goed weer. Het patroon van het EEG verandert met de rijping van de hersenen en biedt daarmee een goede weergave van de hersenontwikkeling.

Bij kinderen die geboren zijn bij een zwangerschapsduur onder de 30 weken toont het EEG patroon een tracé discontinue, dat bestaat uit periodes van EEG activiteit (bursts) een EEG inactiviteit (interburst intervals). Met toenemende postmenstruele leeftijd worden de bursts steeds langer en de interburst intervals steeds korter. Zo ontwikkelt het EEG patroon tot een tracé continue, waarbij er voortdurend EEG activiteit bestaat. Daarnaast veranderen de EEG golven met toenemende postmenstruele leeftijd: de hoogte (amplitude) van de golven wordt steeds lager en de frequentie van de golven wordt steeds hoger. Het beoordelen van een EEG patroon is echter tijdintensief en complex voor neonatologen, die vaak geen experts zijn op het gebied van EEG.

Om het EEG te beoordelen bij kinderen die rond de uitgerekende leeftijd zijn geboren maar zuurstofgebrek tijdens de geboorte hebben doorgemaakt, is een speciale EEG monitor ontwikkeld: de amplitude-integrated EEG ( $\mathrm{aEEG}$ ) monitor. Deze monitor bewerkt het EEG signaal van één kanaal zodanig dat het eenvoudiger is het patroon van het EEG te beoordelen en epileptische activiteit te herkennen. Wanneer men deze aEEG monitor gebruikt bij te vroeg geboren kinderen, ziet men dat ook het aEEG patroon ontwikkelt met de toenemende postmenstruele leeftijd van het kind. Echter, net als het originele EEG patroon wordt ook het aEEG patroon vooral visueel beoordeeld en niet met maat en getal.

Met als doel neurofysiologische hersenrijping (rijping van het EEG en aEEG) eenvoudiger en objectiever vast te leggen, hebben we onderzocht of het mogelijk is het EEG en aEEG van te vroeg geboren kinderen te analyseren door middel van automatische analyse, waarbij een computerprogramma de kenmerken van het EEG en aEEG meet en in getal weergeeft. De belangrijkste vraagstellingen van dit onderzoek zijn dan ook:

1. Is het mogelijk om neurofysiologische hersenrijping van gezonde te vroeg geboren kinderen te onderzoeken door middel van automatische analyse van het EEG en aEEG?

2. Welke van de EEG en aEEG kenmerken die gemeten wordt bij automatische analyse is het meest geschikt om neurofysiologische rijping bij te vroeg geboren kinderen te beschrijven?

Voor deze studie zijn kinderen onderzocht die geboren zijn bij een zwangerschapsduur kleiner 
dan 32 weken, een ongecompliceerd klinische beloop doormaakten en geen neurologische afwijkingen toonden bij een leeftijd van 2 jaar. Bij deze kinderen werden tijdens de NICU opname wekelijks 4 uur durende EEG metingen verricht. Met behulp van (onder andere zelf ontwikkelde) computersoftware werden de EEG en aEEG patronen geanalyseerd. Voor de studie in hoofdstuk 8 zijn ook metingen verricht bij kinderen die geboren zijn bij een zwangerschapsduur groter dan 32 weken.

In hoofdstuk 2 is een literatuurstudie gedaan naar wat bekend is over het EEG van te vroeg geboren kinderen. In dit hoofdstuk wordt de verandering in continuïteit besproken (de ontwikkeling van tracé discontinue tot tracé continue), aanwezigheid van specifieke EEG golven die passen bij een bepaalde leeftijd, ontwikkeling in frequentie-inhoud van de golven (meer laagfrequente golven bij lage postmenstruele leeftijd, golven met hogere frequentie op hogere leeftijd), synchroniciteit (gelijktijdig voorkomen van EEG activiteit in beide hersenhelften) en ontwikkeling van slaapwaakritme in het EEG. Ook wordt hier beschreven dat een achterlopende hersenrijping gecorreleerd is met neurocognitieve problemen op latere leeftijd.

In hoofdstuk 3 is de EEG inactiviteit (interburst intervals) bestudeerd met behulp van de software van een EEG recorder. Interburst intervals zijn automatisch gedetecteerd en gemeten. Ook is het percentage EEG inactiviteit over periodes van 10 minuten gemeten (Interburst-Ratio; IBR). Door middel van de IBR kan tracé discontinue (periodes waarbij EEG activiteit afgewisseld wordt met EEG inactiviteit en dus hoge IBR) en tracé continue (patroon met continue EEG activiteit en nauwelijks EEG inactiviteit en dus lage IBR) automatisch gedetecteerd worden. De lengte van tracé discontinue neemt af met de postmenstruele leeftijd terwijl de lengte van tracé continue toeneemt. De lengte van interburst intervals neemt af met de postmenstruele leeftijd.

In hoofdstuk 4 zijn EEG burst en interburst intervallen gedetecteerd met een zelf ontwikkeld algoritme. Zo hebben we $90 \%$ van de bursts en $80 \%$ van de interburst intervals automatisch kunnen detecteren. De optimale grens voor de automatische detectie van bursts en interburst intervals is $30 \mu$ Volt. Met toenemende leeftijd verandert de lengte van de bursts niet. Zoals ook in het voorgaande hoofdstuk is aangetoond, neemt de lengte van de interburst intervals af.

In hoofstuk $\mathbf{5}$ is het EEG signaal bestudeerd door middel van automatische spectraal analyse. Het EEG patroon bestaat uit verschillende frequentiebanden: delta 1 (0.5-1 Hz), delta $2(1-4 \mathrm{~Hz})$, theta $(4-8 \mathrm{~Hz})$, alfa $(8-13 \mathrm{~Hz})$ en beta $(13-30 \mathrm{~Hz})$. Spectraal analyse (Fourier analyse) is een techniek die een signaal kan ontleden in sinusfuncties van verschillende frequenties. Zo kan de absolute en relatieve bijdrage van de verschillende frequentiebanden aan het gehele signaal (EEG patroon) bepaald worden. Deze bijdrage wordt weergegeven in absolute power $\left(\mu \mathrm{V}^{2}\right)$ en relatieve power (\%). Hoe meer power, hoe meer aandeel van een bepaalde frequentieband. Met toenemende postmenstruele leeftijd nam de absolute power van de delta en theta frequenties af. De relatieve power van delta 1 frequentie nam ook af terwijl de relatieve power van de alfa en beta frequenties toenamen. Deze veranderingen waren het best waar te nemen in het centro-temporale gebied.

Om het EEG nog preciezer te analyseren is in hoofdstuk 6 de spectraal analyse uit hoofdstuk 5 gecombineerd met de burst-detectie software uit hoofdstuk 4. Zodoende is de spectraal analyse alleen op de EEG bursts verricht. Daarnaast is in dit onderzoek, door middel van het analyseren van meer EEG kanalen, nog specifieker gekeken naar het onderscheid in rijping tussen de verschillende 
hersengebieden. Ook in deze studie namen de lagere frequenties af en hogere frequenties toe met de postmenstruele leeftijd. Opvallend is dat de veranderingen het best geobserveerd konden worden in het temporale en frontale hersengebied. Dit komt overeen met de veranderingen die bij MRI onderzoek in deze hersengebieden waargenomen worden.

In hoofdstuk 7 is automatische analyse verricht op het amplitude-integrated EEG (aEEG) patroon. Van het aEEG patroon werd de gemiddelde waarde van de ondergrens (LMA), de bovengrens (UMA) en de bandbreedte (BW) berekend en weergegeven in microvolt. Ook werd het tijdspercentage dat het aEEG patroon discontinue was $(\mathrm{LMA}<5 \mu \mathrm{V})$ berekend. De ondergrens (LMA) van het aEEG patroon nam toe met de postmenstruele leeftijd, terwijl bovengrens (UMA) en bandbreedte (BW) niet veranderden. Het tijdspercentage discontinue aEEG patroon nam af met toenemende postmenstruele leeftijd.

In hoofdstuk 8 is automatische analyse van het aEEG patroon van meerdere kanalen verricht om zo regionale verschillen in het brein te ontdekken. Ook zijn voor deze studie metingen verricht bij kinderen die geboren zijn na 32 weken zwangerschapsduur. De verschillen in aEEG patroon tussen de kanalen waren klein. Ook was er sprake van symmetrie tussen beide hersenhelften. Wel werd gevonden dat de skewness van de data (statistische maat voor hoe data rondom het gemiddelde van de meting verdeeld zijn) veranderde met postmenstruele leeftijd. Opvallend is dat deze waarde, die nooit met het blote oog opgevangen kan worden, erg nauw samenhangt met de posmenstruele leeftijd. Dit toont aan dat met automatische analyse meer aan het aEEG signaal opgemerkt kan worden dan met het blote oog alleen.

In hoofdstuk 9 wordt een kort overzicht gegeven en worden de resultaten bediscussieerd. Het is mogelijk neurofysiologische rijping van te vroeg geboren kinderen te bestuderen door middel van kwantitatieve analyse. EEG metingen zijn ook bij deze kleine kinderen goed mogelijk gebleken.

Er zijn meerdere markers gevonden die neurofysiologische hersenrijping goed kunnen beschrijven zoals interburst interval lengte, aEEG patroon ondergrens (LMA) en skewness van LMA. Deze markers geven de verandering in EEG continuïteit(=activiteit) en de ontwikkeling van slaap-waakritme weer. Daarnaast blijken absolute en relatieve delta power en relatieve alfa en beta power goede markers te zijn. Deze markers geven de ontwikkeling van lage naar hoge frequentie in het EEG weer. We hebben de bestudeerde markers het best waar kunnen nemen in de centrale ( $\left.\mathrm{C}_{3}-\mathrm{C}_{4}\right)$ centro-temporale ( $\mathrm{C}_{3}-\mathrm{T}_{3}$ en $\left.\mathrm{C}_{4}-\mathrm{T}_{4}\right)$ kanalen.

De gevonden markers geven de neurofysiologische hersenrijping goed weer. Echter, de resultaten kunnen nog steeds beïnvloed worden door artefacten (stoorsignalen in het signaal) die niet allemaal gefilterd. Bovendien wordt het EEG niet alleen beïnvloed door de rijping van het brein, maar ook door het slaap-waakritme en de klinische toestand van het kind (glucose spiegels en carbondioxide spiegels beïnvloeden bijvoorbeeld ook het EEG). Om automatische EEG analyse bij vroeggeborenen verder te verbeteren zal nog betere detectie van artefacten en slaap-waaktoestand moeten plaatsvinden, waarbij bijvoorbeeld ook gebruik gemaakt wordt van ademhalingssignaal, hartritmesignaal en videomonitoring.

Ook kan door het ontwikkelen van een zogenaamd multivariate model onderzocht worden of de combinatie van meerdere markers een nog preciezer beeld geeft van hersenrijping dan iedere individuele marker apart. Daarnaast is het interessant om neurofysiologische ontwikkeling (EEG 
onderzoek) te relateren aan structurele ontwikkeling (MRI onderzoek).

Aangezien de aEEG monitor al veel gebruikt wordt op de NICU, zouden de algoritmes uit dit onderzoek geïmplementeerd kunnen worden in deze monitor. Op deze wijze wordt er extra objectieve en kwantitatieve data gegenereerd die gebruikt zou kunnen worden voor onderzoek van vroeggeborenen.

Het monitoren van het brein bij vroeggeborenen kan helpen bij het ophelderen van de vraag welke factoren gunstige en ongunstige invloed hebben op de hersenontwikkeling. Dit zou van nut kunnen zijn bij het ontwikkelen van medische en verpleegkundige interventies die gericht zijn op de hersenontwikkeling. Als automatische analyse van het EEG verder ontwikkeld wordt, zou neurofysiologische "brain monitoring" in de toekomst een waardevol hulpmiddel kunnen zijn bij het beschermen van de hersenontwikkeling van vroeggeborenen. 



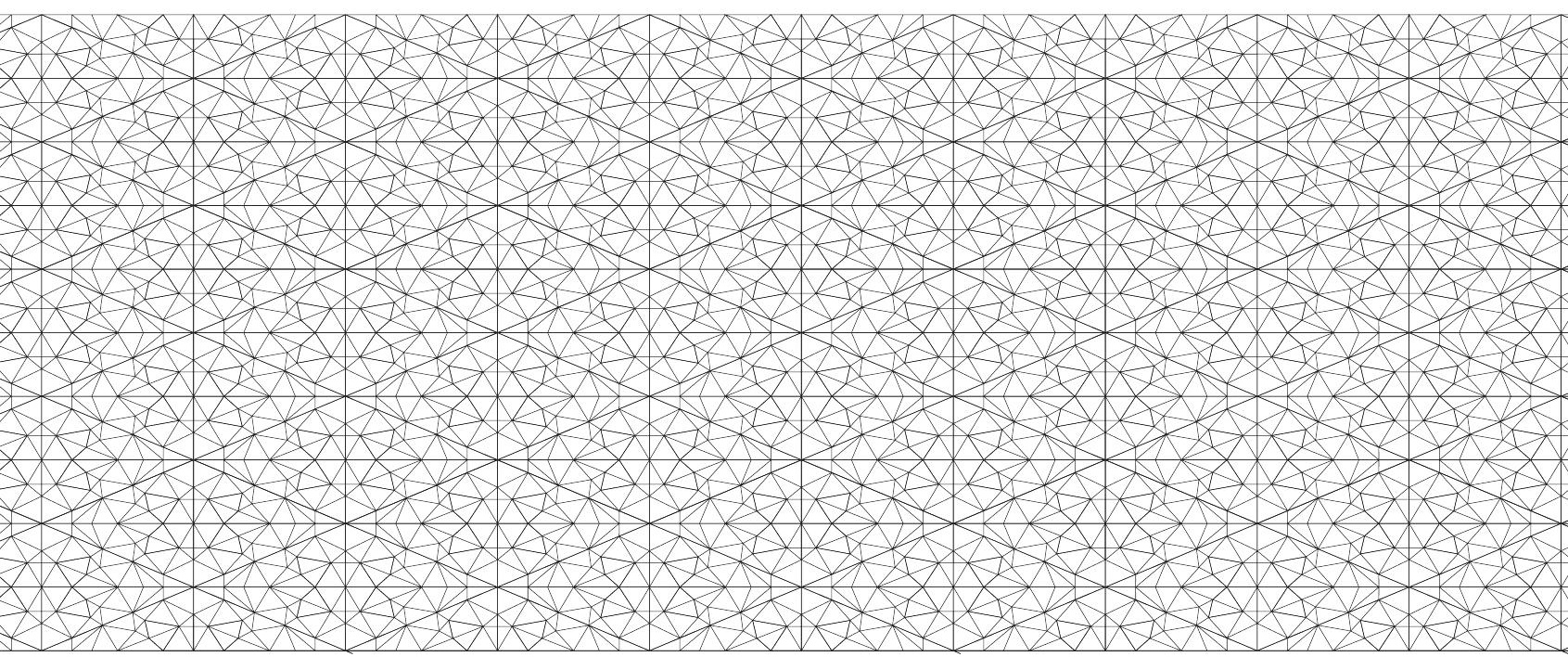




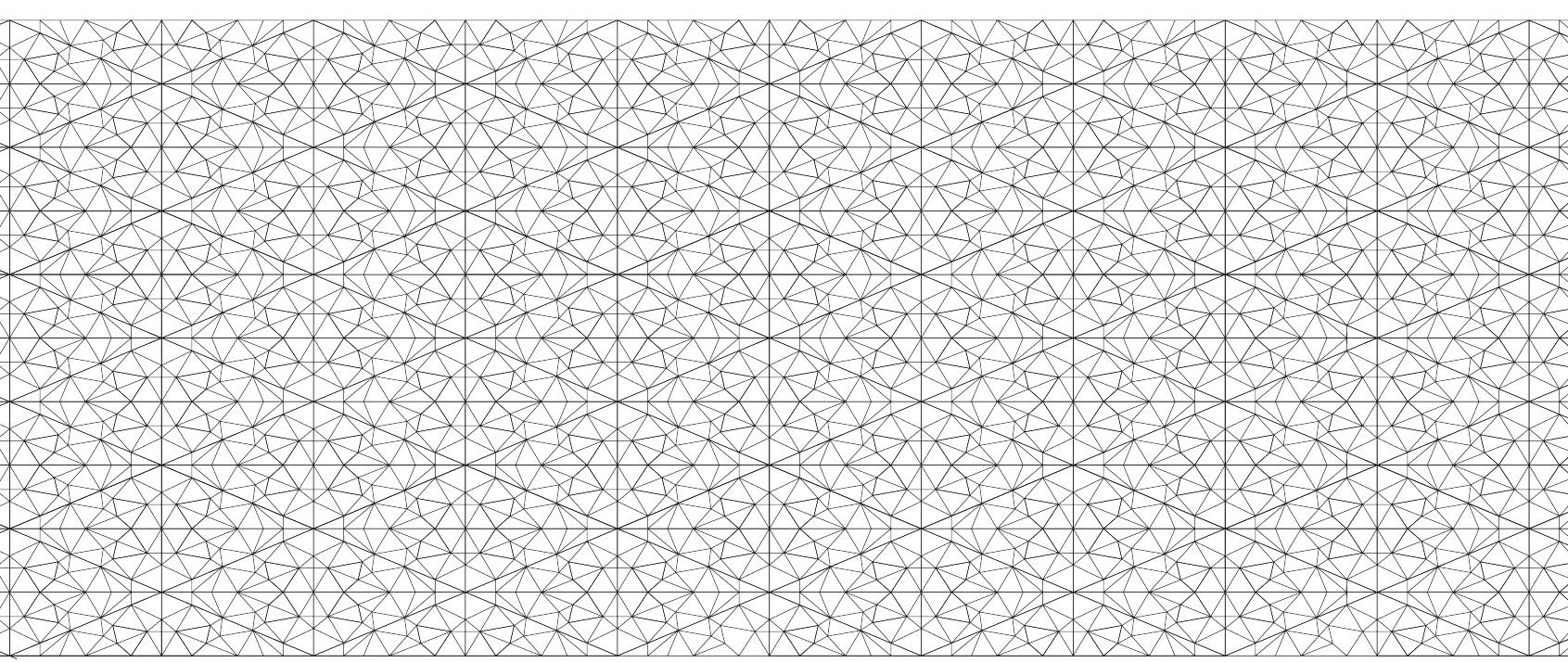

Dankwoord 


\section{Dankwoord}

Als promovendus die onderzoek doet in een perifeer ziekenhuis en daarnaast zijn opleiding tot kinderarts doorloopt, ben ik bijzonder veel mensen bijzonder veel dank verschuldigd.

Ten eerste wil ik de ouders en kinderen hartelijk bedanken voor de deelname aan het onderzoek. Zonder jullie belangenloze medewerking is klinisch onderzoek onmogelijk.

Ook wil ik de leescommissie, bestaande uit Prof.dr. W.H. Mess, Prof.dr. A.F. Bos, Prof.dr. T. Delhaas, Dr. J. Nicolai en Prof.dr. L.S. de Vries bedanken voor het boordelen van mijn manuscript.

Vervolgens wil ik Professor Sidarto Bambang Oetomo bedanken. Beste Sidarto, ik weet nog als de dag van gisteren dat je me vroeg om een voordracht te verzorgen over therapeutische hypothermie bij asfyxtische pasgeborenen. Ik beet me in dit onderwerp vast en ontdekte de schoonheid van het neonatale brein. Deze voordracht leidde uiteindelijk tot het opstarten van dit promotieonderzoek in Veldhoven. Zonder jouw visie op het belang van monitoren van het premature brein en de waarde van onderzoek voor een perifere NICU, was dit onderzoek er nooit gekomen. Je gaf me veel ruimte om mijn eigen weg te gaan, waarbij je de hoofdlijn van het onderzoek bewaakte en altijd weer de puntjes op de i wist te zetten. Ook heb je een cruciale rol gespeeld bij het bemachtigen van een opleidingsplaats voor de kindergeneeskunde. Daar ben ik je nog iedere dag dankbaar voor!

Daarnaast bedank ik Professor Carlos Blanco. Mede dankzij u werden de eerste wetenschappelijke samenwerkingsverbanden tussen Veldhoven en Maastricht gelegd en kreeg ik de mogelijkheid me verder te bekwamen in de kindergeneeskunde.

Professor Zimmermann. Beste Luc. Jij nam het stokje over van Professor Blanco. Jij begeleidde het onderzoek verder en werd mijn Maastrichtse promotor zonder het onderzoek zijn Veldhovense karakter af te nemen. Daarbij ben je van grote steun geweest bij de afronding van het project. Veel dank daarvoor.

Professor Kramer. Beste Boris. Toevallig trof je mij in de kliniek met de handen in het haar over een artikel. Je las het artikel en zag onmiddellijk wat goed was en wat ontbrak. Dat was de basis voor jouw promotorschap. Vervolgens heb je me veel bijgebracht over kritisch denken, formuleren en schrijven. Je hebt een feilloos instinct voor wetenschap en hebt me veel fijne kneepjes kunnen bijbrengen. Daarnaast ben je een prettig mens in de omgang. Je bent een rasoptimist, oprecht betrokken met een enorme motivatie. Ook nu blijf je me motiveren om verder te gaan in de wetenschap en me te ontwikkelen tot neonatoloog. De stage in Keulen, die jij mogelijk gemaakt hebt, is daar een voorbeeld van. Dank daarvoor Boris!

Co-promotor Peter Andriessen. Beste Peter. Aan jou ben ik verreweg de meeste dank verschuldigd. Jij bent de spil van dit promotieonderzoek geweest. Vanaf het begin heb je me begeleid, bijgestuurd 
en gesteund in het onderzoek. Altijd objectief, kritisch, punctueel, soms prikkelend en vooral enthousiast begeleidde je het onderzoek. Ik heb altijd het gevoel gehad dat je precies wist wat voor vlees je met mij in de kuip had. Je gaf ruimte aan mijn sterke punten en mijn zwakke kanten drukte je hardhandig de kop in. Deze aanpak ging gepaard met aanmoedigingen, humor, vaderlijke adviezen en soms een streng woord. Je bent een uitstekend wetenschapper en daarnaast een voorbeeld voor me in de kliniek. Ook heb ik het altijd gewaardeerd dat je ook op persoonlijk vlak geïnteresseerd bent en weet wat me beweegt in het leven. Ik kijk dan ook met groot plezier op ons project terug en ik zie uit naar verdere samenwerking in de toekomst, waarbij ik nog veel van je hoop te leren.

Ad Smets, Teamleider KNF MMC Veldhoven. Beste Ad, met name bij de praktische uitvoering van de metingen was jij een spil in dit onderzoek. Je hebt me veel tips and tricks van het EEG meten meegegeven. Ondanks je eigen drukke werkzaamheden was je bereid om me te helpen en vond je oplossingen voor praktische problemen. Ik heb dan ook vele uren op de KNF doorgebracht: voor vragen en hulp, maar ook voor de gezelligheid, want een bezoek aan Ad gaat nooit voorbij zonder koffie en een goede mop.

Charlotte Lommen, Medical Engineer TU Eindhoven/Máxima Medisch Centrum. Beste Charlotte. Jij bent van groot belang geweest bij de opzet van het onderzoek. Je hebt me wegwijs gemaakt in de complexe EEG meetapparatuur en je deed dit op zodanige wijze dat een technische no-no het ook echt begreep: met geduld en goede communicatieve skills. Ook heb je veel meegedacht bij het opzetten van de eerste analyse methoden. Daarmee heb je aan de wieg gestaan van dit boekje. Hierbij bedank ik ook Loes Ruijs, Student BMT van de TU Eindhoven, die onder begeleiding van Charlotte de basis heeft gelegd voor een Premature EEG analyse toolbox. Een toolbox die zijn basis vond in het uitstekende afstudeerproject van Rick Vullings.

Chris Peters, Klinisch Fysicus MMC Veldhoven. Beste Chris, mijn grote steun en toeverlaat bij de analyses van het EEG. Als ik een idee had, luisterde je, ging aan de slag met Mathlab en toverde in no-time een algoritme. Vervolgens wist je het toverwerk ook nog duidelijk uit te leggen. Dank daarvoor!

Jaco Pasman, Klinisch Neurofysioloog Universitair Medisch Centrum St.Radboud Nijmegen. Beste Jaco. Je hebt me ingewijd in de geheimen van het neonatale EEG, zowel bij het verrichten als bij het interpreteren ervan. Daarnaast had je een goed idee welke aspecten van het neonatale EEG zich zouden lenen voor kwantitatieve analyse. Dit heeft mij voor een groot gedeelte op het goede spoor gezet. Ik kijk met plezier terug op mijn bezoeken aan het Nijmeegse, die naast leerzaam en motiverend ook altijd gezellig waren.

Vivianne van Kranen-Mastenbroek, Klinisch Neurofysioloog MUMC Maastricht. Beste Vivianne. Ook van jou heb ik zeer veel geleerd over het Neonatale EEG. Daarnaast heb je samen met Jaco vele EEGs geannoteerd. Dit was een enorme klus, maar heeft uiteindelijk geleid tot verdere ontwikkeling 
van automatische EEG algoritmes.

Marijke van Aken, Imke Maartens en Tessa Wassenberg. Toen ik Veldhoven voor Heerlen verruilde, hebben jullie op fantastische wijze de draad opgepakt door metingen en analyses te verrichten. Zo zijn jullie van grote waarde geweest voor een van de manuscripten.

Danilo Gavilanes, Neonatoloog MUMC Maastricht. Als expert op het gebied van de neonatale neurologie heb je inhoudelijk veel input bij het schrijven van mijn laatste manuscripten gehad. Dank!

René Richards, Accountmanager Viasys Healthcare. Godzijdank lag het Máxima Medisch Centrum op de route. Zo kon je vaak op weg van of naar huis ons uit de brand helpen als we tegen een probleem aanliepen. Dank voor je immer snelle, vriendelijke en goede hulp.

Ward Jennekens, Klinisch Fysicus, MMC Veldhoven. You were the right man at the right time in the right place with the same problems! Onze beide onderzoeken gingen door een moeilijke fase (die van mij door gebrek aan technische ondersteuning, bij jou luisterden de ratten niet) toen Peter Andriessen ons op hetzelfde pad bracht. Onze goede samenwerking heeft geleid tot het slagen van beide projecten. Hierbij wil ik ook Carola van Pul, Klinisch Fysicus MMC Velhoven, die Ward mede begeleid heeft bedankten. Tevens wil ik Marjolein Engels, afstudeerstudente bij Ward Jennekens, bedanken voor haar inzet.

Professor Vles, Hoogleraar Kinderneurologie MUMC. U wil ik hartelijk danken voor uw input en het delen van uw visie bij het opzetten van het onderzoeksproject. Het blijft jammer dat we in een later stadium te weinig gebruikt hebben gemaakt uw kennis en ervaring.

Titia Katgert, Medisch Psycholoog van het MMC Veldhoven. Bedankt voor de nauwgezette followup van de kinderen uit de studie.

Saskia Houterman, Epidemioloog van het MMC Veldhoven. Bedankt voor je onmisbare statistische ondersteuning.

Veel dank ben ik verschuldigd aan de Raad van Bestuur van het Máxima Medisch Centrum Veldhoven, die bereid was om als niet academisch ziekenhuis een groot onderzoeksproject te financieren en mij als arts-onderzoeker aan te nemen.

Daarnaast wil ik zeker ook het verpleegkundig team van de NICU van het MMC Veldhoven bedanken. Jullie waren altijd bereid om met me mee te denken en mee te werken om de EEG metingen te laten slagen. Zo zijn jullie van van onschatbare waarde geweest voor dit project. Het Máxima mag trots zijn op zo'n groep gemotiveerde en betrokken verpleegkundigen! Ik heb altijd met veel plezier met jullie samengewerkt en ik verheug me dan ook om volgend jaar weer als fellow bij jullie aan de slag te gaan. 
Voor hun interesse in dit project en de prettige samenwerking wil ik de kinderartsen van het MMC Veldhoven en van het MUMC Maastricht bedanken. In het bijzonder: Twan Mulder, mijn opleider. Piet Leroy, mijn mentor (ook op het gebied van taalpurisme). Feico Halbertsma, met wie ik enkele case-reports geschreven heb en die altijd bijzonder geïnteresseerd was in mijn project en tot slot Thilo Mohns, die me inzicht heeft verschaft in het denken en werken van De Duitser (wat onmisbaar is geweest in Keulen).

Tevens wil ik mijn voormalig collega arts-assistenten en collega fellows bedanken voor de prettige samenwerking. In het bijzonder mijn maatjes uit de Heerlense en Veldhovense tijd: Sanne Hammer, Merel Klaassens, Marijn Schuurman en Annelies van de Ven. Ook wil ik roostercommissie-(lot) genoot Barbara Werrij bedanken voor de fijne samenwerking.

Lina Meisen van Neondot.com. Dank voor de prachtige lay-out van mijn boekje!

\section{Tot slot de mensen uit mijn privé leven:}

Mijn studievriendengroep bestaande uit Nanne de Boer, Tom van Boxsel, Joost van der Hart en Maarten van de Sande. Helaas zien we elkaar veel te weinig, maar ik geniet nog volop van de momenten dat we samen zijn. Onvoorstelbaar dat zo'n clubje ongeregeld goed terecht kan komen en zo'n brave burgers geworden zijn...... Gelukkig doen we minstens een keer per jaar alsof dat niet zo is.

Bas Liethoff. Vriend en collega-barman uit de Preuverij uit ver vervlogen tijden. Dank voor je verfrissende vriendschap! Je blijft mijn cultureel en politiek geweten. Ik zie je veel te weinig, maar een bezoek aan jou in Berlijn staat garant voor een spectaculair weekend. Maar meest dankbaar blijf ik dat je Sanne aan me hebt voorgesteld.

In het bijzonder mijn beste vrienden en paranimfen Nanne de Boer en Joost van der Hart. Ik ben blij en trots dat jullie achter me staan! Nanne, jij ging me voor in het promotietraject en hebt me als ware vriend gesteund en geadviseerd. Je bent een voorbeeld voor me in je optimisme en doorzettersmentaliteit. Je originaliteit, humor, bourgondische instelling en relativerende blik op het leven zorgen nog altijd voor mooie avonden als we elkaar bezoeken.

Lieve Joost, oude roommate en studentenbar-maat. Vriend die me kent als zijn broekzak. Dank voor de jarenlange vriendschap gevuld met warmte, goede gesprekken en veel, heel veel lol. Onze studiereis India was onvergetelijk en heeft me gevormd als mens en arts. Nog steeds ben ik je dankbaar daarvoor!

Mijn moeder. Lieve Mamma. Jarenlang heb je ons gezin alleen 'gedraaid'. Naast je fulltime werk en opleiding in avonduren heb je ervoor gezorgd dat wij nooit iets tekort kwamen. Ik heb je nooit iets anders zien doen dan werken of voor ons zorgen. Nooit heb ik je chagrijnig gezien of horen klagen. Daarnaast vond je toch iedere dag de tijd om met me te praten over wat me bezighield, 
me te steunen en te motiveren. Hoe jij je wegcijfert voor anderen is ongelofelijk en eigenlijk ongehoord. Nog steeds blijf je ons steunen en sta je altijd voor ons klaar met daad, maar ook met wijze raad. En daarbij ben je ook nog eens een fantastische oma. Dank mam!

Mijn stiefvader, Wim. Sinds je bij ons kwam wonen heb je mijn interesses gezien en gevoed. Na 3 maanden wist ik als 10 jarig jochie van alles over communisme, meridianen en atomen, kon ik uitleggen waarom het kabinet gevallen was en had ik een abonnement op KIJK. Daarnaast vertelde je vol enthousiasme over je eigen werk als huisarts. Hiermee heb je mijn interesse voor geneeskunde en wetenschap gewekt. Dit en je doorgaande steun en motivatie hebben er voor gezorgd dat ik ben waar ik nu ben. Het is fantastisch om te zien wat voor een lieve opa je bent voor Liselotte!

Mijn vrouw, Sanne. Lieve Sanne, mijn engel. Mijn grootste geluk in mijn leven is dat ik jou heb ontmoet. Je bent een fantastische vrouw. Je steun, zorg en onvoorwaardelijke liefde hebben ertoe geleid dat ik dit boekje heb kunnen schrijven, maar ook (laten we eerlijk zijn) dat ik iedere dag met twee dezelfde sokken op mijn werk verschijn, mijn sleutels terug vind en enigszins gezond eet. Je humor en je positieve instelling zorgen ervoor dat ons leven samen heerlijk is. Je wijsheid, intelligentie en brede interesse maken elke dag en gesprek boeiend. Daarnaast ben je een voorbeeld voor me in je werklust en ambitie om de wereld voor kinderen beter te maken. lk vrees dat ik vaker aan jou heb gevraagd om te stoppen met werken om 'iets leuks' te gaan doen dan andersom. Je bent daardoor een gevierd juf. De karrevracht aan cadeaus en bedankbrieven van ouders getuigen daar ieder jaar van. Naast dit alles ben je ook nog eens een geweldige oermoeder voor onze Liselotte. Ik weet niet hoe je het voor elkaar krijgt maar je doet het toch. Ik hou van je en ik ben ontzettend trots op je.

Lieve Liselotte. My pride and joy. Mijn kleine onderzoeker. Als ikjou vol enthousiasme en temperament de wereld zie ontdekken en vol vreugde in actie zie in het experimenteel laboratorium dat onze woonkamer heet, weet ik weer waarom onderzoek -ondanks de stress van zo'n boekje - zo leuk is! lk verheug me erop om samen met jou de wereld verder te (her)ontdekken. 



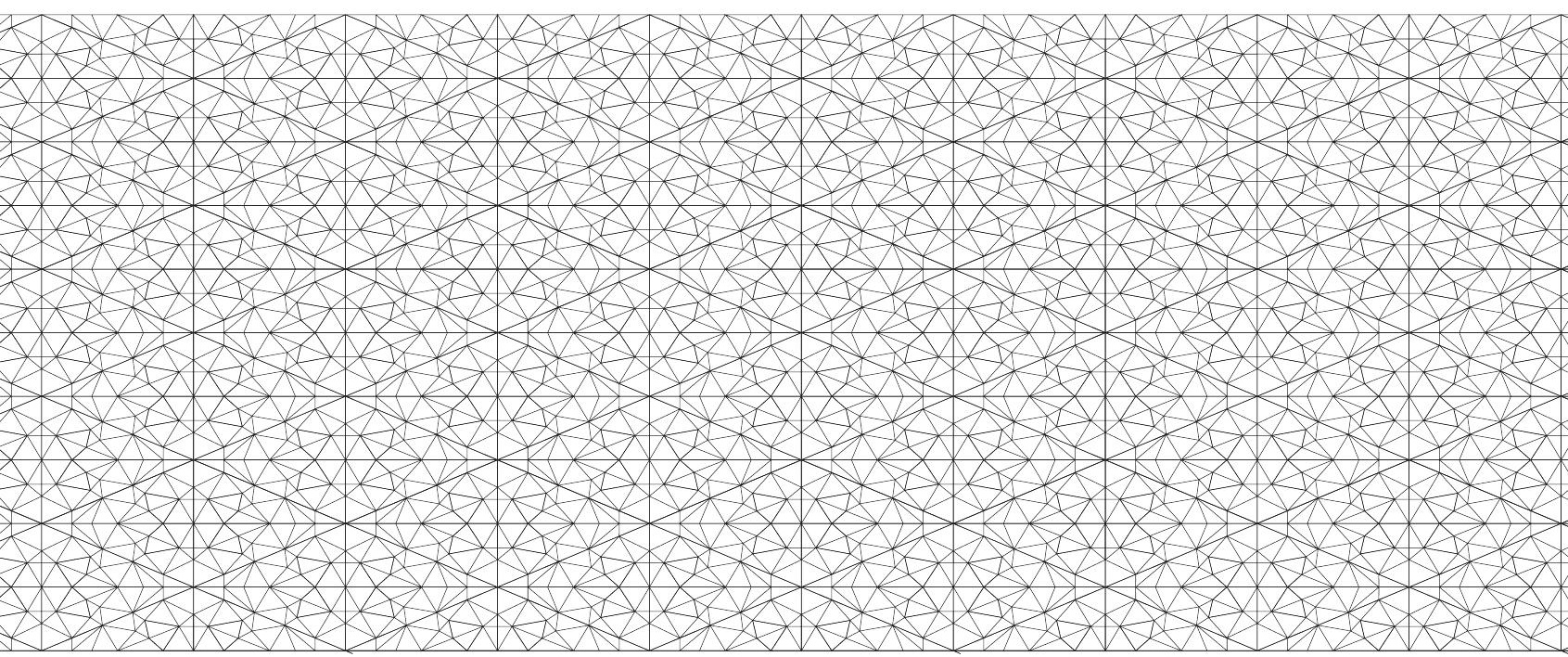




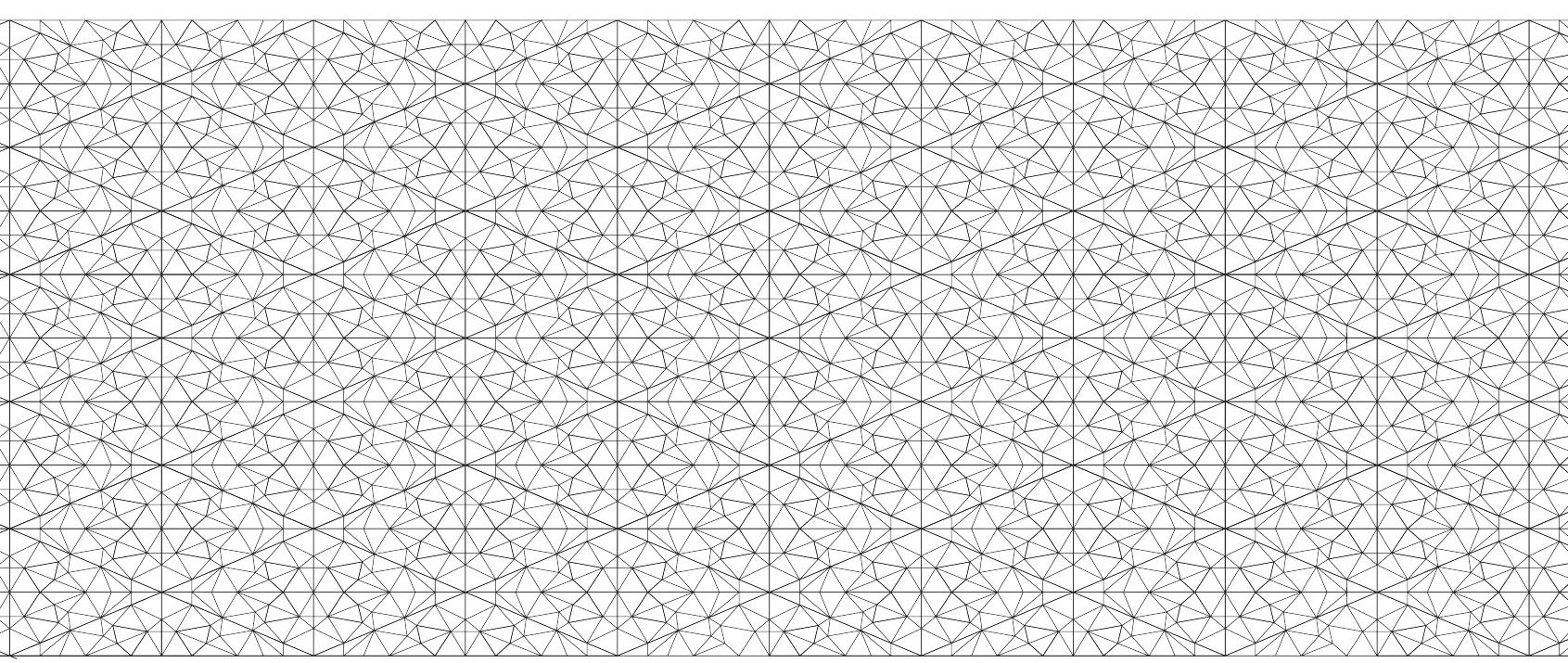

\section{Curriculum Vitae}





\section{Curriculum Vitae}

Hendrik Johannes Niemarkt werd op 16 februari 1978 geboren in Heerlen. In 1996 voltooide hij het Gymnasium aan het College Sittard. Na een jaar Geneeskunde gestudeerd te hebben aan de Katholieke Universiteit Leuven, startte hij in 1997 met de opleiding Geneeskunde aan de Universiteit Maastricht. Deze werd in 2003 afgerond. In 2004 en 2005 werkte Hendrik als AGNIO in het Máxima Medisch Centrum te Veldhoven, waar hij eind 2005 begon met dit promotieonderzoek. In ditzelfde ziekenhuis was hij in 2006 en 2007 werkzaam als arts-onderzoeker.

In 2008 startte hij met de opleiding Kindergeneeskunde in het Atrium Medisch Centrum Heerlen (opleider: Dr. J.E. Dankert-Roelse) en vanaf juli 2009 in het Maastricht Universitair Medisch Centrum (opleider: Prof. dr. L.J.I. Zimmermann). Het laatste half jaar van zijn opleiding heeft hij zijn profileringsstage doorlopen in het Perinatalzentrum van de Uniklinik Köln (begeleiders: Prof. dr. B. Roth / Dr. A. Kribs). In juli 2012 begon hij aan het fellowship Neonatologie in het Maastricht Universitair Medisch Centrum en Máxima Medisch Centrum Veldhoven.

Hendrik is getrouwd met Sanne Roumen. Ze hebben een dochter, Liselotte. 


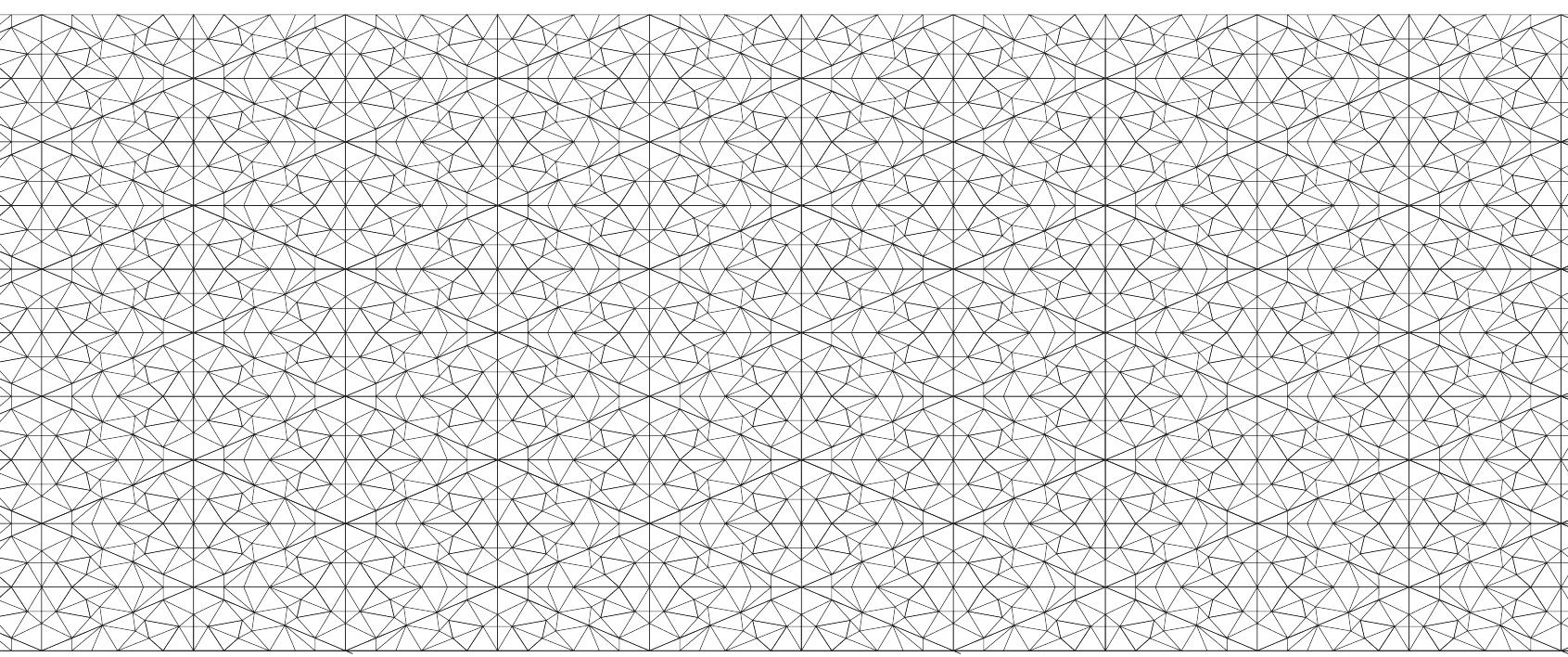




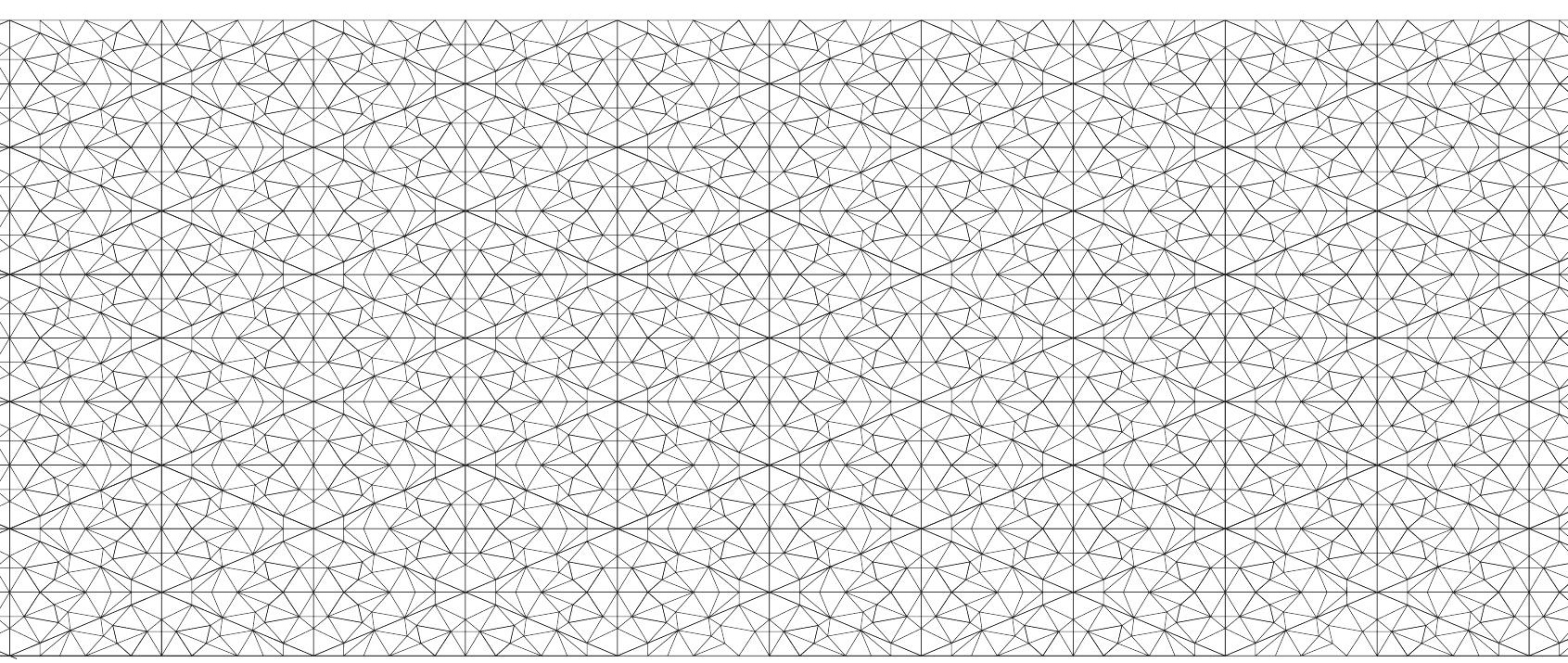

List of publications 


\section{Thesis}

Niemarkt HJ, Andriessen P, Pasman J, Vles JS, Zimmermann LJ, Oetomo SB.

Analyzing EEG maturation in preterm infants: The value of a quantitative approach. Journal of Neonatal-Perinatal Medicine 2008;1(3):131-144.

Niemarkt $\mathrm{HJ}$, Andriessen P, Peters CHL, Pasman JW, Blanco CE, Zimmermann LJ, Bambang Oetomo S.

Quantitative analysis of amplitude-integrated electroencephalogram patterns in stable preterm infants, with normal neurological development at one year.

Neonatology 2010;97(2):175-182.

Niemarkt HJ, Andriessen P, Peters CHL, Pasman JW, Zimmermann LJ, Bambang Oetomo S. Quantitative analysis of maturational changes in EEG background activity in very preterm infants with a normal neurodevelopment at 1 year of age.

Early Human Development 2010;86(4):219-224.

Niemarkt HJ, Jennekens W, Pasman JW, Katgert T, van Pul C, Gavilanes AWD, Kramer BW, Zimmermann LJ, Bambang Oetomo S, Andriessen P.

Maturational changes in automated EEG spectral power analysis in preterm infants.

Pediatric Research 2011;70(5):529-534.

Jennekens W, Ruijs LS, Lommen CML, Niemarkt HJ, Pasman JW, van Kranen-Mastenbroek VHJM, Wijn PFF, van Pul C, Andriessen P.

Automatic burst detection for the EEG of the preterm infant.

Physiological Measurement 2011;32(10):1623-1637.

Niemarkt HJ, Jennekens W, Maartens IA, Wassenberg T, van Aken M, Katgert T, Kramer BW, Gavilanes AW, Zimmermann LJ, Bambang Oetomo S, Andriessen P.

Multi-channel amplitude-integrated EEG characteristics in preterm infants with a normal neurodevelopment at two years of corrected age.

Early Human Development 2012;88(4):209-216.

Jennekens W, Niemarkt HJ, Engels M, Pasman JW, van Pul C, Andriessen P.

Topography of maturational changes in EEG burst spectral power of the preterm infant with a normal follow-up at 2 years of age.

Clinical Neurophysiology 2012. [Epub ahead of print] 
Rosias PP, Robroeks CM, Niemarkt HJ, Kester AD, Vernooy JH, Suykerbuyk J, Teunissen J, Heynens J, Hendriks HJ, Jöbsis Q, Dompeling E.

Breath condenser coatings affect measurement of biomarkers in exhaled breath condensate. Eur Respiratory Journal 2006;28(5):1036-1041.

Niemarkt HJ, Halbertsma FJ, Andriessen P, Bambang Oetomo S.

Amplitude-integrated electroencephalographic changes in a newborn induced by overdose of morphine and corrected with naloxone.

Acta Paediatrica 2008;97(1):132-134.

van Os E, Niemarkt HJ, Verreussel MJ, Cruysberg JR, Bok LA, Spruijt L.

Twee pasgeborenen met congenitale aniridie: noodzaak van genetisch onderzoek.

Nederlands Tijdschrift Geneeskunde 2008;152(10):569-573.

Andriessen P, Struis NC, Niemarkt H, Oetomo SB, Tanke RB, Van Overmeire B.

Furosemide in preterm infants treated with indomethacin for patent ductus arteriosus.

Acta Paediatrica 2009;98(5):797-803.

Schuurman M, van Waardenburg D, Da Costa J, Niemarkt H, Leroy P.

Severe hemolysis and methemoglobinemia following fava beans ingestion in glucose-6phosphatase dehydrogenase deficiency: case report and literature review.

European Journal Pediatrics 2009;168(7):779-82.

Jennekens W, Dankers F, Janssen F, Toet M, van der Aa N, Niemarkt H, van Pul C, de Vries L, Andriessen $\mathrm{P}$.

Effects of midazolam and lidocaine on spectral properties of the EEG in full-term neonates with stroke.

European Journal Paediatric Neurolology 2012. [Epub ahead of print]

Niemarkt $\mathrm{H}$, Andriessen $\mathrm{P}$, Halbertsma FJ.

Artefacts in the amplitude-integrated EEG background pattern of a full-term asphyxiated neonate caused by diaphragm spasms.

BMJ Case Reports 2012. 


\section{Book Chapter}

Niemarkt HJ, Andriessen P.

Monitoring brain development in preterm infants: the value of automated analysis of the electroencephalogram (EEG).

In: Neonatal Montoring Technologies: Design for Integrated Solutions. Editors: Wei Chen, Sidarto Bambang Oetomo and Loe Feijs. Publisher: IGI Global Hershey 2012. 



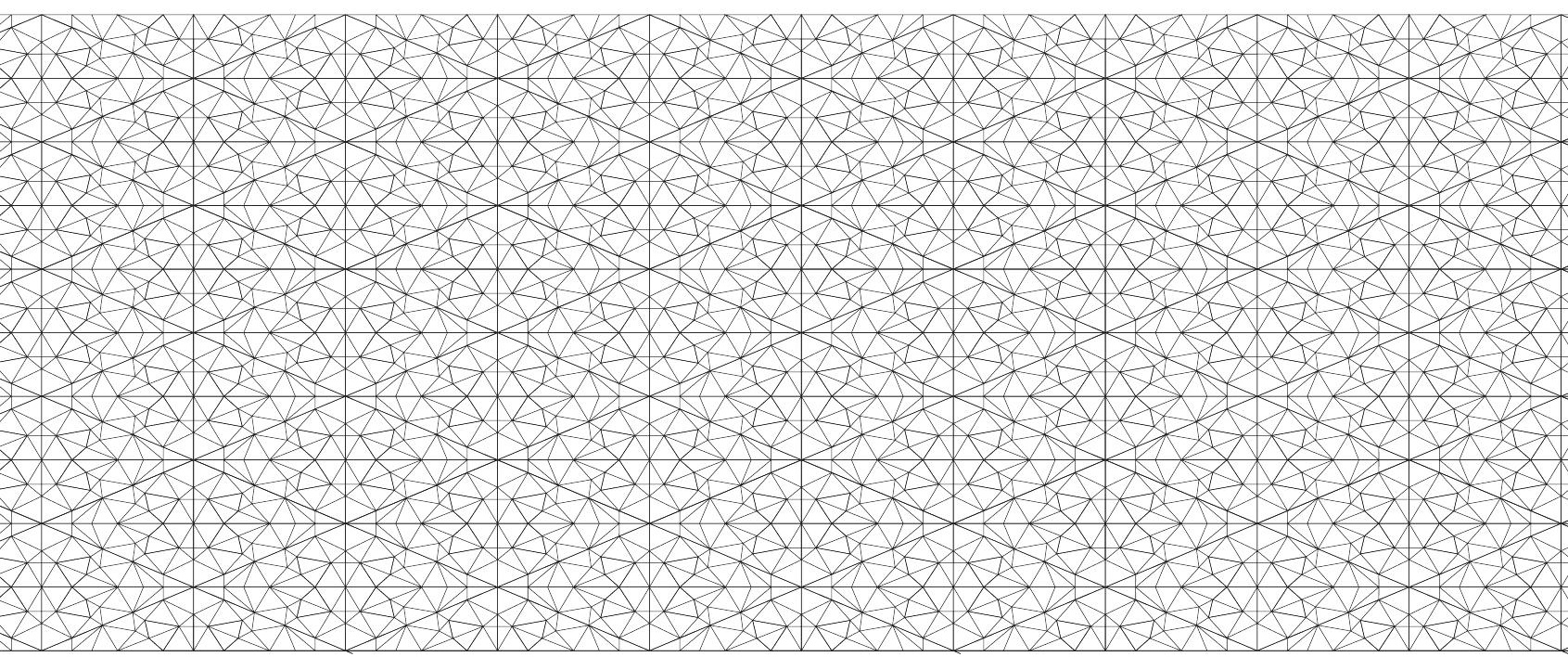




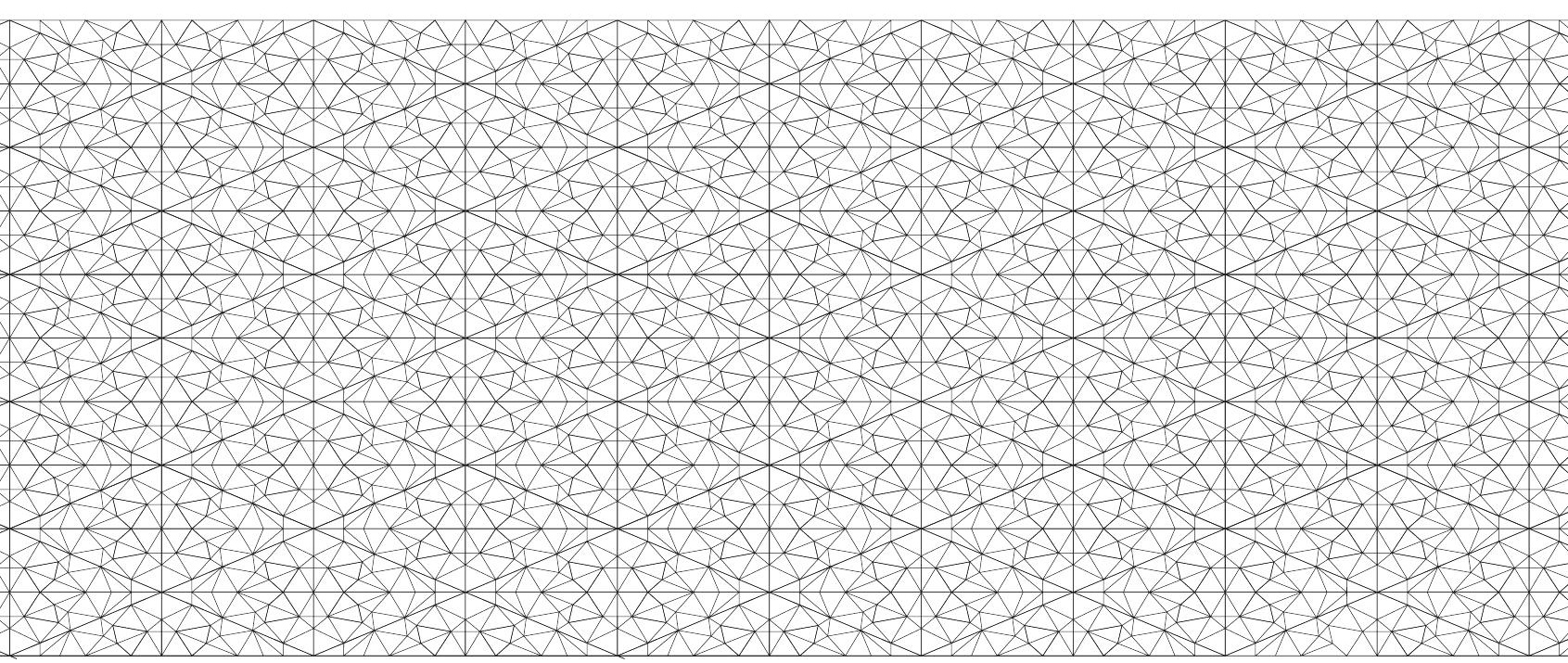

List of co-authors 


\section{List of co-authors}

\section{Dr. P. Andriessen}

Neonatal Intensive Care Unit, Máxima Medical Centre, Veldhoven, The Netherlands

\section{M. van Aken}

Neonatal Intensive Care Unit, Máxima Medical Centre, Veldhoven, The Netherlands

Department of Pediatrics, Maastricht University Medical Centre, Maastricht, The Netherlands

\section{Prof. dr. S. Bambang Oetomo}

Department of Industrial Design, Eindhoven University of Technology, Eindhoven, The Netherlands Neonatal Intensive Care Unit, Máxima Medical Centre, Veldhoven, The Netherlands

\section{Prof. dr. C.E. Blanco}

Department of Pediatrics, Division of Neonatology, Maastricht University Medical Centre, The Netherlands

National Children's Research Centre, Our Lady's Children's Hospital, Crumlin, Dublin, Ireland

\section{Engels}

Hogeschool Zuyd, Department of Biometrics, Heerlen, The Netherlands

\section{Dr. A.W. Gavilanes}

Department of Pediatrics, Division of Neonatology, Maastricht University Medical Centre, The Netherlands

\section{Dr. W. Jennekens}

Department of Clinical Physics, Máxima Medical Centre, Veldhoven, The Netherlands Department of Applied Physics, Eindhoven University of Technology, Eindhoven, The Netherlands

\section{T. Katgert}

Department of Medical Psychology, Máxima Medical Centre, Veldhoven, The Netherlands

\section{Prof. dr. B.W. Kramer}

Department of Pediatrics, Division of Neonatology, Maastricht University Medical Centre, The Netherlands

\section{Dr. V.H. van Kranen-Mastenbroek}

Department of Clinical Neurophysiology, Maastricht University Medical Centre, Maastricht, The Netherlands 


\section{C.M. Lommen}

Neonatal Intensive Care Unit, Máxima Medical Centre, Veldhoven, The Netherlands

Department of Clinical Physics Laboratory, Radboud University Nijmegen Medical Centre, Nijmegen, The Netherlands

\section{I.A. Maartens}

Neonatal Intensive Care Unit, Máxima Medical Centre Veldhoven, The Netherlands

Faculty of Health, Medicine and Life Sciences, University of Maastricht, Maastricht, The Netherlands

\section{Dr. J.W. Pasman}

Department of Clinical Physics, Máxima Medical Centre, Veldhoven, The Netherlands

\section{Dr. C.H.L. Peters}

Máxima Medical Centre, Department of Clinical Physics, PO Box 7777, 5500 MB Veldhoven, The Netherlands

\section{Dr. C. van Pul}

Department of Clinical Physics, Máxima Medical Centre, Veldhoven, The Netherlands

\section{L.S. Ruijs}

Department of Biomedical Engineering, Eindhoven University of Technology, Eindhoven, The Netherlands

\section{Prof. dr. J.S. Vles}

Department of Child Neurology, Maastricht University Medical Centre, Maastricht, The Netherlands

\section{T. Wassenberg}

Neonatal Intensive Care Unit, Máxima Medical Centre, Veldhoven, The Netherlands

Faculty of Health, Medicine and Life Sciences, University of Maastricht, Maastricht, The Netherlands

\section{Prof. dr. P.F. Wijn}

Department of Clinical Physics, Máxima Medical Centre, Veldhoven, The Netherlands

Department of Applied Physics, Eindhoven University of Technology, Eindhoven, The Netherlands

\section{Prof. dr. L.J. Zimmermann}

Department of Pediatrics, Division of Neonatology, Maastricht University Medical Centre, The Netherlands 
